KíK 4223

März 1987

\title{
Informatikanwendungen im Umweltbereich
}

A. Jaeschke Institut fuir Datenverarbeitung in der Technik B. Page Universităt Hamburg Fachbereich Informatik

\section{Kernforschungszentrum Karlsruhe}



Kernforschungszentrum Karlsruhe

Institut für Datenverarbeitung in der Technik

Projekt Schadstoffbeherrschung in der Umwelt

Universität Hamburg

Fachbereich Informatik

KfK 4223

Informatikanwendungen im Umweltbereich

Herausgeber:
A. Jaeschke
B. Page

Kernforschungszentrum Karlsruhe $\mathrm{GmbH}$, Karlsruhe 
Als Manuskript vervielfältigt

Für diesen Bericht behalten wir uns alle Rechte vor

Kernforschungszentrum Karlsruhe $\mathrm{GmbH}$ Postfach 3640, 7500 Karlsruhe 1

ISSN 0303-4003 
Im Kernforschungszentrum Karlsruhe wurde am 30.September und 1. Oktober 1986 ein Kolloquium zum Thema

$$
\text { "Informatikanwendungen im Umweltbereich" }
$$

veranstaltet. Teilnehmer und Vortragende kamen aus Forschung, Wirtschaft und Verwaltung. Die Vorträge des Kolloquiums sind im vorliegenden Band zusammengestellt.

\section{Abstract}

A colloquy on

"Uses of Informatics in Environmental Protection"

was organized at the Karlsruhe Nuclear Research Center on September 30 and October 1, 1986. The participants and the lecturers came from research, industry, and administration. This volume is a compilation of the papers read at the colloquy. 
Informatikanwendungen auf dem Umweltsektor

Realisierungsstand und Forschungsperspektiven

B. Page

Konzeption für das Umweltinformationssystem Baden-Württemberg (UIS)

D. Bartl

Das Umweltplanungs- und Informationssystem des Bundes UMPLIS;

Aufgaben, Hoffnungen und Grenzen

J. Seggelke

Ein Schalenmodell für den Einfachdialog verschiedener Benutzergruppen beim Umweltinformationssystem UMPLIS

J. Seggelke

Verzeichnisgesteuerte Faktendatenbank am Beispiel des Informationssystems

für Umweltchemikalien, Chemieanlagen und Störfälle (INFUCHS)

M. Stopp

DV-gestützte Umweltplanung auf der Basis von Geländedaten

W. Gillessen

Entwicklung eines Informationssystems

"Umweltbelastung und Umweltprobleme"

E. Reichardt

Rechnergestützte Kontrollsysteme für Sonderabfälle

Anforderungen und Realisierungsbedingungen

D. Brune 
Konzeption und Implementierung eines datenbankgestützten Auswertungssystems für Luftmeßdaten

A. Hansen, B. Page, U. Zacharias

Ansätze für Expertensysteme in der Umweltverträglichkeitsprüfung

A. Schwabl

Konzepte zur Unterstützung der Modellbildung und Simulation am Beispiel der Modellbildung auf dem Umweltsektor

L. Hilty

Interaktive Informations- und Entscheidungshilfe Systeme für industrielles Risiko, Gefahrengut und Sonderabfallmanagement

K. Fedra

Wissensbasierte Simulation eines Ökosystems

S. Becker, G. Hille, M. Ramlow 


\section{Vorwort}

Im Verlauf seiner Geschichte hat sich der Mensch in der natürlichen Umwelt seinen Lebensraum erobert, die Umwelt nach seinen Bedürfnissen umgestaltet und sich seine künstliche Wohn- und Arbeitswelt aufgebaut. Nicht erst heute wird dem Menschen dabei bewußt, daß die Umwelt durch sein ständiges Eingreifen und Ausbeuten irreversiblen Schaden nimmt, und zwar in solchem. Maße, daß Umwelt und gewonnener Lebensraum davon ernstlich bedroht sind.

Die Umwelt als Problembereich und die Notwendigkeit Umweltschutz zu betreiben, das sind Fakten, mit denen heute jeder Bürger konfrontiert ist, über die er laufend (allerdings mit verschiedenen Vorzeichen und unter unterschiedlichem Blickwinkel) durch Fernsehen, Funk und Presse informiert wird. Umweltschutz setzt aber exakte Kenntnis voraus über die Umwelt selbst, über die Belastungsfaktoren und über die wechselseitigen Wirkungsmechanismen. Diese Kenntnis existiert heute jedoch nicht ausreichend und jeder fortschritt auf diesem Gebiet deckt nur neue Kenntnislücken auf. Wissenschaft, Politik und Wirtschaft kommen zu gegensätzlichen Einschätzungen und Lösungsvorschlägen. Eine einheitliche Gesamtsicht der Probleme ist nicht einmal in ihren Grundzügen skizziert.

Von wissenschaftlicher Seite besteht (speziell bei jungen Wissenschaftlern) große Bereitschaft, sich der anstehenden Probleme anzunehmen. Dabei ist außer Diskussion, daß alle Sparten der Wissenschaft beitragen können und müssen. Insbesondere ist auch die Informatik herausgefordert, denn die Information über die Umwelt und über ihre Veränderung durch den Menschen und durch die Technik ist die Basis aller umweltschutz-bezogenen Aktivitäten und Maßnahmen. Dabei ist die Vielfalt und Menge der anfallenden Informationen kaum zu überschauen.

Zustand und Geschehen in der Umwelt werden durch physikalische, chemische, meteorologische und biologische Daten beschrieben. Es werden räumlich verteilte Informationen benötigt; aus den kleinen begrenzten Gebieten der Biotope, aus größereren klimatisch, wirtschaftlich und politisch begrenzten Regionen und aus der Gesamtheit der Biosphäre. Diese Informationen sind außerdem zeitlich verteilt und kennzeichnen vergangene und aktuelle Zustände.

Ohne die technische Hilfe von automatischen Meßeinrichtungen, Datenfernübertragungen und Prozeßdatenverarbeitung - von Netzen, die ganze Regionen überziehen, ist die Beschaffung dieser Informationen nicht zu bewältigen. Die vielen dezentral ermittelten Informationen müssen in umfassenden und fachspezifischen Informationssystemen zusammengefügt werden. Sie müssen in ihren vielfältigen Beziehungen zueinander zu einem Rechenmodell verarbeitet werden, um ein der jeweiligen Fragestellung gemäßes Abbild des Systems "Umwelt" zu erhalten. Ohne maschinelle Informationsverarbeitung, Archivierung und Aktualisierung der Information in Datenbanken, mit der Möglichkeit des automatisch problemspezifischen Zugriffs auf die Daten, wäre dies nicht machbar. Von Bedeutung sind dabei die Möglichkeiten, die moderne grafische Methoden bieten und deren Fähigkeiten zu kompakten Informationsdarstellungen genutzt 
werden. Ein wichtiges Hilfsmittel bei der Lösung von Umweltproblemen stellt die rechnergestützte Modellbildung und Simulation dar. Sie ist ein noch viel zu wenig genutztes Instrument in der Analyse komplexer Relationen und Abhängigkeiten sowie bei Planungsprozessen und Entscheidungsfindungen. Eine bedeutende Erweiterung der Möglichkeiten wird hier zukünftig der Einsatz von Methoden der Künstlichen Intelligenz, speziell der Expertensysteme, bringen. Das Anwendungsspektrum ist dabei derzeit noch nicht abschätzbar.

Der Einsatz der Informationstechnik läßt sich damit als wesentliche Voraussetzung für den Umweltschutz ansehen.

Die Situation ist derzeit gekennzeichnet durch eine Vielfalt von Aktivitäten und Ansätzen in eingeschränkten Problembereichen mit eingeschränkten Zielsetzungen. So realistisch und aus der Dringlichkeit der Problemsituation wohl begründet diese Ansätze auch sind, so bilden sie kaum mehr als einzelne Steine in einem Mosaik, da ein Gesamtkonzept sich derzeit noch nicht klar abzeichnet.

Ein Schritt auf dem Weg hierhin ist die Intensivierung der Kommunikation zwischen den Wissenschaftlern. In diese Richtung zielt das vom Institut für Datenverarbeitung in der Technik (IDT) und dem Arbeitsschwerpunkt Technik, Mensch, Umwelt (TMU) des Kernforschungszentrums Karlsruhe veranstaltete Kolloquium vom 30.9.-1.10.1986 zum Thema

"Informatikanwendungen im Umweltbereich", dessen Vorträge im vorliegenden Band zusammengestellt sind.

\section{Dr. A. Jaeschke}

Kernforschungszentrum Karlsruhe GmbH

Institut für Datenverarbeitung in der Technik

Prof. B. Page

Universität Hamburg

Fachbereich Informatik 


\section{Einführung}

Man mag fragen, warum sich das Kernforschungszentrum Karlsruhe (Kf́K) nun mit Umweltschutz beschäftigt. In der Öffentlichkeit ist kaum bekannt, daß in der Kerntechnik von Beginn an auch Umweltschutztechnik entwickelt wurde. So wird seit langem im Laboratorium für Aerosolphysik und Filtertechnik (LAF) Abgas- und Abscheidungstechnik mit zugehöriger Grundlagenforschung entwickelt. Ergebnisse dieser Arbeiten schlugen sich in den Abgasreinigungs- und Filteranlagen von Kernkrafiwerken und Wiederaufarbeitungsanlagen nieder. Ebenso müssen Abwässer aus nuklearen Anlagen aufbereitet werden, bevor sie in die kommunalen Gewässer eingeleitet werden dürfen. Nuklear-chemische Verfahren zur Schadstoffbeseitigung sind in den Instituten für Heiße Chemie (IHCH) und Radiochemie (IRCH) entwickelt worden. Schadstoffausbreitungsmodelle sind in den Hauptabteilungen Sicherheit (HS) und Angewandte Systemanalyse (AFAS) sowie in unserem Institut für Datenverarbeitung in der Technik (IDT) erstellt und berechnet worden.

In der Kerntechnik ist der Brennstoffkreislauf einschließlich Entsorgung einmalig. Er besteht aus einem geschlossenen Kreislauf von der Brennstoffherstellung über Brennstoffverbrennung im Kraftwerk und Wiederaufarbeitung des abgebrannten Brennstoff́s zur Rückführung verwertbarer Stoffe sowie zur Lagerung konzentrierter Abfälle. Innerhalb dieses Kreislaufs werden die verschiedenen Materialien und Schadstoffe verfolgt und bilanziert. Ich möchte meinen, daß dieser Kreislauf als Vorbild für den nicht-nuklearen Bereich dienen sollte.

Dieses in der Kerntechnik erworbene umfassende Wissen und die reiche Erfahrung sollen nun für die dringenden Probleme der Entsorgung im nichtnuklearen Bereich eingesetzt werden. Als ein Beispiel der Übertragung des kerntechnischen Wissenschatzes in den nicht-kerntechnischen Bereich sei die prototypische Rauchgas- und Entstickungsanlage genannt, die im Rhein-Dampfkraftwerk Karlsruhe erprobt wird. Zur Konzentration der F+E-Arbeiten im Kernforschungszentrum Karlsruhe wurde der neue Arbeitsschwerpunkt Technik-Mensch-Umwelt ins Leben gerufen.

Das $K f K$ ist auch deshalb für diese $F+E$-Arbeiten bestens geeignet, da es schon seit langem interdisziplinär projektorientiert gearbeitet hat und über wichtige Disziplinen wie Chemie, Biologie, Meteorologie, Meßtechnik und Informatik in einem Hause verfügt.

Das Institut für Datenverarbeitung in der Technik (IDT) hat durch seine Arbeiten zur Wiederaufarbeitung, Lagerung und Kernmaterialüberwachung seit über 10 Jahren Erfahrung bei der Erstellung von Informationssystemen für Materialverfolgung, Anlagenüberwachung und Prozeßührung. Außerdem hat es mathematische Modelle zu Risiko- und Störfallanalysen u.a. in der Abgasreinigung aufgestellt und numerisch simuliert. 
Diese Erfahrungen haben wir bereits als Einstieg in die nicht-nukleare Entsorgung durch erste Ansätze für das baden-württembergische Ministerium für Ernährung, Land- und Forstwirtschaft für Umweltschutz (MELUF) und für die Landesanstalt für Umweltschutz (LfU) genutzt. So wurden von uns Konzepte (Systemarchitektur und Software) sowie Kostenabschätzungen für verteilte DV-Systeme zur Materialverfolgung bei einer Sondermülldeponie und zur Verwaltung der umfangreichen Daten der Wasserwirtschaftsämter in Baden-Württemberg entworfen. Wir sind jetzt dabei, für eine Pilot-Müllverbrennungsanlage des Laboratoriums für Isotopentechnik (LIT) die Prozeßführung zur Minimierung der Schadstoffabgabe und für das Abfallmanagement in Baden-Württemberg ein Informations-Expertensystem zu entwickeln.

Bei unseren Arbeiten wurde uns bewußt, daß wir einer enormen Breite von Problemstellungen gegenüberstehen, die wir nur durch Zusammenarbeit mit kompetenten Fachleuten aus den verschiedenen Bereichen des Umweltschutzes bewältigen können. Das Kolloquium ist daher von lebendigem Gedankenaustausch und gegenseitiger Unterstützung zur Verfolgung gleichgerichteter Ziele geprägt - nämlich unsere Lebensumwelt mit den besten informationstechnischen Mitteln zu erhalten und zu verbessern.

Prof. Dr. H. Trauboth

Leiter des Instituts für Datenverarbeitung in der Technik

Kernforschungszentrum Karlsruhe $\mathrm{GmbH}$ 
Informatikanwendungen auf den Umweltsektor -

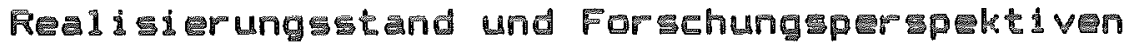

B. Page

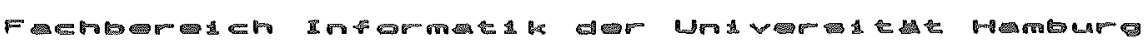

Zusammenfassung:

Dieser ubersichtsbitrag berakt sich it der stand der Informatikanwendungen i Uwweltberaich sowohl aus der sicht der Ppaxis als ach hinsichtich wölicher forschungspichtungen. Entwicklungsstand und tendenzen des coupuereinsatzes in der Uhweltschutzpraxis werden anhand einer evipischen studie in den staritichen Uneltbehöden referiert. Anschisebend werden einige forschungsperspektiven rü Unweldanwendungen aus den sicht der Angewndten Inforatik augezeigt wobei auch muf eigene Beiträge aus ussere Arbeitsbereich verwiesen wird. Schisilich werden die vorausetzungen fü die Entwicklung hin ze eine eigenstandigen forschungsgebiet "Unwelt-Intor atik" diskutient

GLIEDERUNG

1. Einführusa und ubersicht

2. Realisierunasstand des Conoutereinsatzes in Ulimeltbereich

2. I Fachinhalt1 iche schwerpunkte der uneltbezogensn computranendungen

2.2 Systertechnische Aspekte

2.3 Bemertung der Du-Unrrage in den Unweltbehören

3. Forschunaspersoektiven fü die Anqewandte Inforegik in Anwendungsbereich brwelt

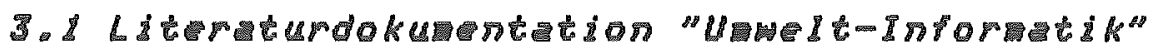

3.2 Wicht-konventionel1E Datenbankanwendungen

3.3 Nethoden- und Nodellbanksystere

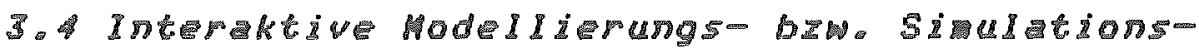
systere

3.S Expertensysethe

- Schubberschtunas Aur den Heq zu einer "unweltInforadik"? 


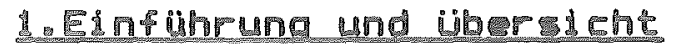

Die ständig wachsende Nachfrage nach umueltrelevanten Informationen erFordert zunehmend den Einsatz moderner Informationsverarbeitungsmethoden. Daher setzt sich der Computerainsatz in den mit Umweltaufgaben vorrangig befaßten staatlichen Bahörden auch immer stärker durch. Auch wenn der Umweltbereich bisher ain sehr spezialisierter DU-Anuendungsbereich gebliaben ist - Standardsortware beispielsweise genört auf diesem Sektor aher $z u$ den Ausnahmen (vgl. /HPPB5/, S. 32) -, so ist angesichts steigender Umultausgaben zukünetig durchaus mit einer zunahme von dessen Marktbedeutung zu Eechnen. Der überwiegende Teil der umweltspezifischen Computeranwendungen stellt Praxisentwicklungen dar. Als Anwendungsgebiet Eür die Infarmatik stießs der Umweltbereich bis data nur aur garinges forschungsintarassa, obwohl bereits vor fast 10 Jahren eine erste fachtagung

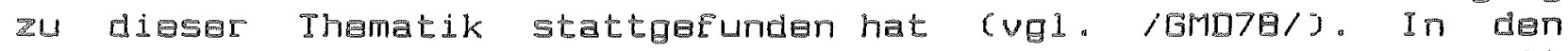
Folgenden Jahren sind jedoch von Informatiksaite nur varainzelt Publikationen über Umweltanwendungen erschienen cygl. $\mathbb{Z} . B$. /KLNBO/,/STEBO/,/BUSB2/,/PANB2/,/GRPB3/,/PASB3/ ader/STOB3/3. bei denen es sich gräbtenteils um Praxisarbeiten handelte. In letzter zeit scheint sich jedoch oin zunehmendes Interesse auch aus dem wissenschaftlichen Bereich am Umweltsektor als Informatik-Anwendungsgebiet $z u$ entwickeln, wie sich an kürzlichen Veranstaltungen cöffentliche Ausstellung und vortragsreihe "Chancen mit Chips" mit einem Rahmenthema "Chips und Umuelt" der Siemens AG im Dktober 1984 in Berlin, siehe auch /SIEB4/, Tagung "EDU Eür Umweltschutz und Energiewirtschaft" Nov. 1985 in üsterreich, siehe /ADUBS/ oder Seminarvortragsreihe am FB Informatik der Universität Hamburg im Sommer 1985, aus der der Sammelband /PAGBGa/ hervorgagangen ist) souil an mehreren aktuellen Publikationen (vgl. /APPB5/, /BOS85/, /BOS86/, /HABBG6/,/KUHB5/, /MGKB6/, oder /SYDBG/) und schließlich auch an diesem Kolloquium ablesen läßt. Einige Ubersichtsarbeiten $z u$ Informatikanwendungen auf dem Umweltsektor sind ebenfalls in neuerer Zait erschienen cugl. /HITBY/, /HIPBS/, /HPPES/, /HIPGGa/, /PILB5/ oder/ZIEBG/).

In ersten Teil dieses Beitrages (Kap. 2) soll zur Analyse des Realisierungsstandes der umweltbezogenen InEarmatikanwendungen in der Praxis auf die Ergebnisse oiner Studie über den DU-Einsatz in den Umweltbehörden der Bundesländer sowia des Umweltbundesamtes (UBA) zurückgegriffan werden, dia der Autar im Auftrag das UBA 1985/86 durchgeführt hat. Im zweiten Iail des Beitrages (Kap.3) werden einige Forschungsperspektiven aus der Sicht der Angewandten Informatik für den Anwendungsberaich Umwelt skizziert wie der Aufbau einer computergestützten Literaturdokumentation "Umwalt- 
Informatik", wie Nicht-kanyentionel le Datenbankanuandungen Für den Umualtberaich, Methodenbankarchitekturan Eür Auswertungssystame, Modellbank- und interaktive Modeliderungs- bzw. Simulationssystama Für Umueltmodelle sowie Expertansystame für Umubltanuandungen. Dabei wird auch auF oigene Baiträge aus dem Arbitsberbich "Anwendungen der Informatik in Geistes- und Naturwissenschaften" am FB Informatik der Universitat Hamburg verwiasen.

In der Schlußbetrachtung wird der Frage nachgegangen, walcho Ansprüche an ein eigenständiges Fachgebigt "Umuelt-Informatik" Für die Zukunft zu stellen sind.

\section{Real isierunasetand des Computereinsatzes im Unwel bereich}

Auf Initiative des Bund-/Länderarbeitskreises Umueltinformationssysteme wurde vorn Unweltbundesame im Rahmen des Imbormacions- und Dokumentarionssyetrme Ummel e eine Studie an den Autor vergeben, in der die umueltbezogenen DU-Anwendungen in den Unweltbehörden dar Bundesländer und des Umueltbundesamtes auf der Basis einer UmErage hinsichtich Eachinhalticher und systemtechnischer Kriterien erfaßt und analysiert werden sollten.

Uorrangige Ziele diesar DU-Umrrage waren U.a.

- die erforderliche Informationsbasis für oinen Softwargaustauseh bzw. einen Erfahrungsaustausch über konkrete DU-Entwicklungen unter Bundes- und Länderbehörden zu schaffen;

- zur Vermeidung kostenaufwendiger Doppelentwicklungen beizutragen;

- den stand des DU-Einsatzes in den Umweltbehórden zu dokumentieren;

- und schließlich umweltspezifische DU-Entwicklungsschwarpunkte und -trends aus der Praxis für die Zukunft aufzuzeigen.

Die Durchëhrung der Umerage in den Umweltbehörden erfolgte in der Zeit van Juni 1985 bis April 1986, und zwar sowohl in form einer Fragabogenerhebung als auch durch persónliche Befragungen (Interviews). An der Umfrage uurden insgesamt mehr als 50 Behórden mit Umweltaurgaben aus Bund und Ländern beteiligt. Es sind Angaben zu ca. 360 DU-Uerfahren eingegangen. Die Ergebnisse der Umfrage wurden in einem Bericht gtudie über DV-Anwendungen in den Unweltbehörden des Bundes und der Länder ausführlich dokumentiert (auf ca. $300 \mathrm{5.3}$ und werden einer breiten interessierten Fachoffentlichkeit zugänglich gemacht /PAG86c/. In der Studie sind sowohl statistische Gesamtaussagen als auch Einzelangaben zu den DU-Uerfahren enthalten. Zur Charakterisierung des Realisierungsstandes des Computereinsatzes auf dem Umweltsektor in der Praxis werden hier die wichtigsten statistischen Ergebnisse der Umfrage wiedergegeben. 
2.1 Fachinhaltiche Schwergunkte der unweitberogenen Comouteranwendunaen

Die Abb. 1 zeigt die Verteilung der Computeranwendungen auf die verschiedenen Umwertefachgebiete, wobei die Abkürzungen der Fachgebiete der Umweltklassifikation des Umweltbundesamtes entsprechen:

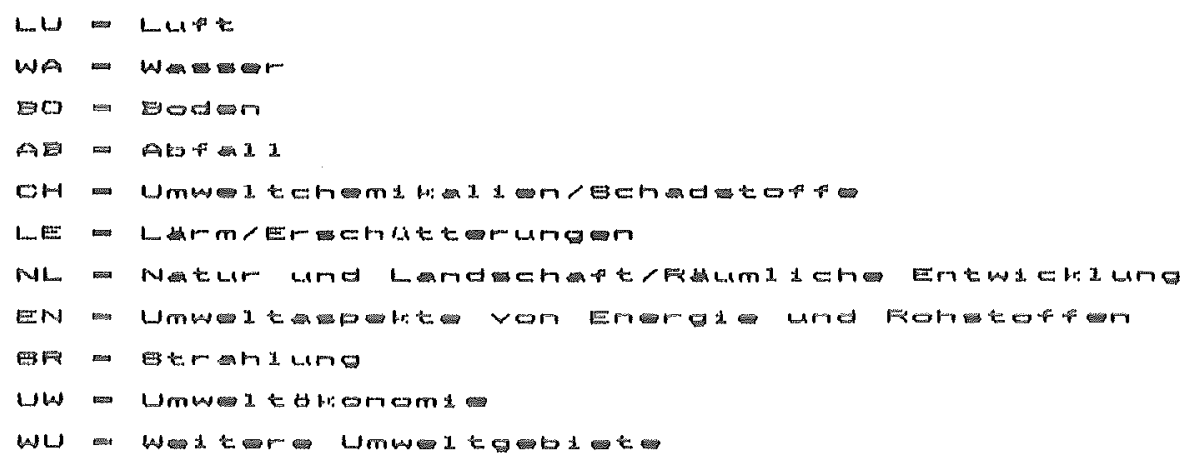

Unter "Sonstige" sind nicht Eachspezifische Anwendungen $C z . B$. Statistikj sowie Anuendungen in nicht umweltspezifischen Fachgebieten ( $z . B$. Ioxakologie) zusammengefaßt.

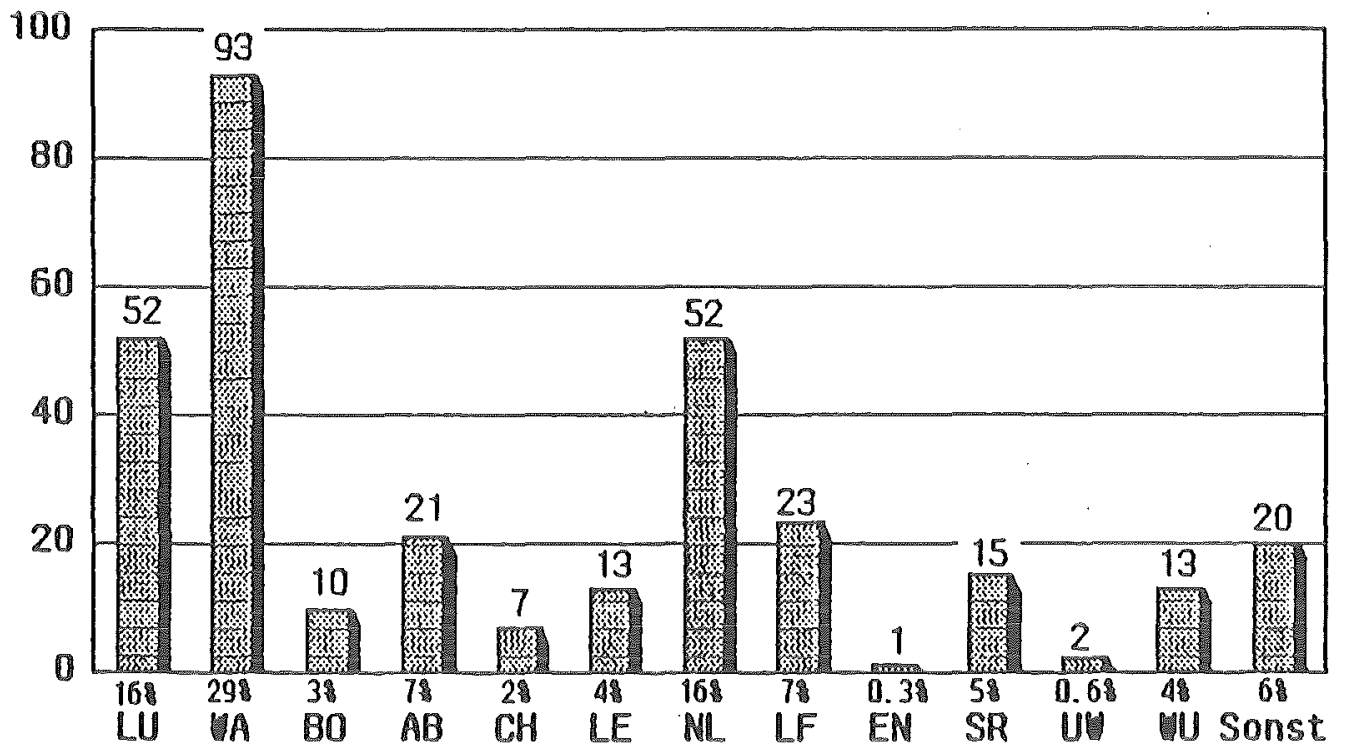

Abb. 1: DV-Verfahren in den verschiedenen Umweltfachgebieten 
Schwerpunkte des Computereinsatzes liegen in der wasserwirtschaft sowia in den Bereichen LuEt bzw. Natur und Landschaft.

Computer werden in den Umweltbehörden für sehr unterschiedliche Problemstellungen eingesetzt. Diese können in Ealgende Anwandungsklassen eingeordnet werden (vgl, auch $A b b .2)$ :

- ErfasseniMessen (Z.B. Luftmeßnetze)

- Datenverwaltung (z.B. Umweltliteraturdatenbanken mit Elexiblen Anfragemöglichkeitenl

- Auswertung (z.B. Emissionsschätzungen aus rechnergestütztem Emissionskataster)

- Modellrechnung/Simulation (z.B. Ausbreitungsrechnung'

- Graphik (z.B. Biotopkartierung)

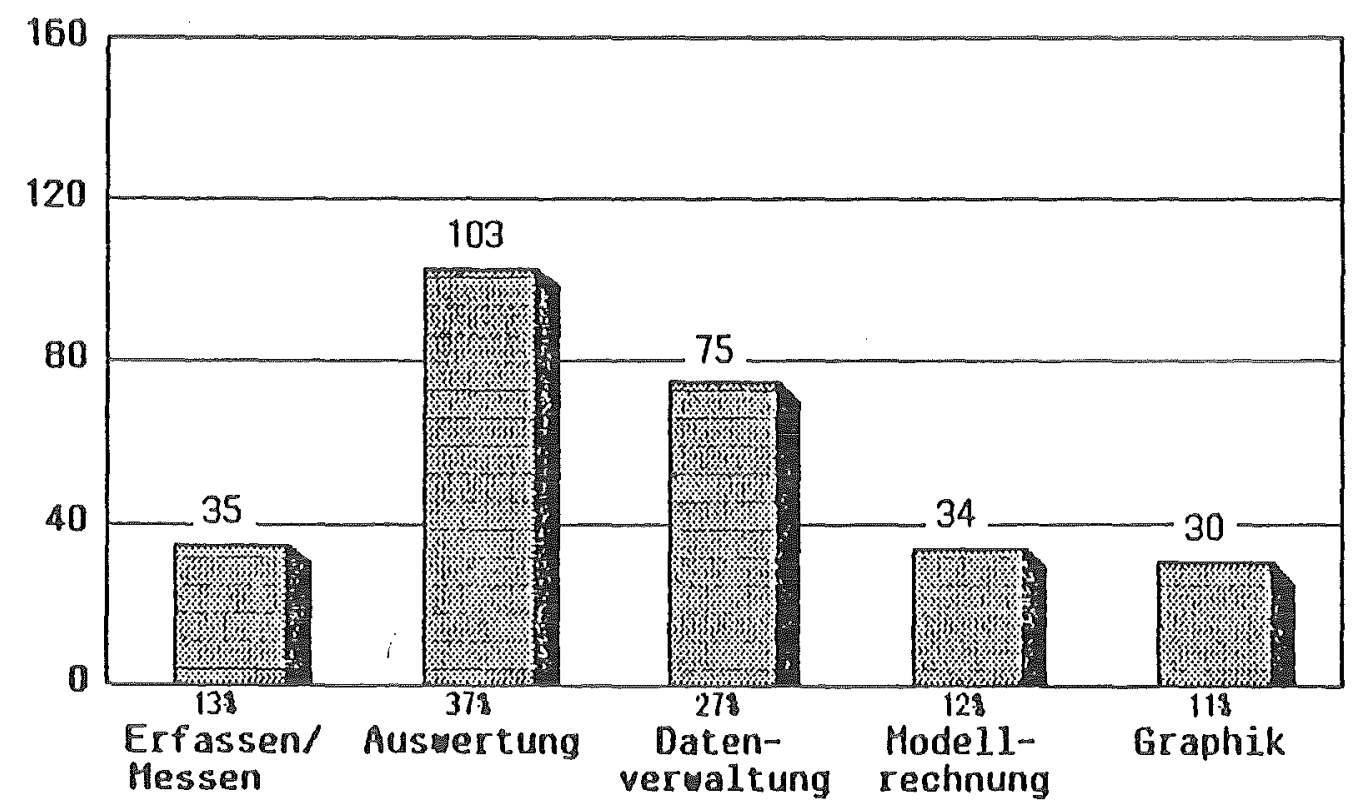

Abb. 2: DV-Verfahren nach Anwendungsklassen 
Dabei kännen jedoch häufig einzelne Computaranuendungen varschigdenen Klassen glaichzaitig zugerordnet werden, de sie unterschiedliche Anuendungsfunktionen augfühen $\mathfrak{C Z} B$. ist aine Landschaftsdatenbank mit Funktionen zUE Kartenerstellung unter dan Klassen "Datenverwaltung" und "Graphik" zu berücksichtigend.

In der Folgenden Tabelle sind die Eachlichen Schwerpunkte der Computeranwendungen in den einzelnen Bundesländern und dem Umueltbundesamt $z u$ erkennen, wobei in der zweiten spalte auch angegeben ist, wieviele der Uerfahren bereits realisiert sind. Die Abkürzungen der Bundesländer sind selbsterklärend, bei den Fachgebieten sind die obigen Bezeichnungen (vgl. Abb.1) verwendet.

\begin{tabular}{|c|c|c|c|c|c|c|c|c|c|c|c|c|c|c|c|}
\hline \multirow{2}{*}{ Land } & \multirow{2}{*}{$\begin{array}{l}\text { Anzahl } \\
\text { gemeldete } \\
\text { Anwendungen }\end{array}$} & $\begin{array}{l}\text { davon } \\
\text { realisierte } \\
\text { Anwendungen }\end{array}$ & \multicolumn{13}{|c|}{$\begin{array}{l}\text { realisierte oder geplante Anwendungen } \\
\text { nach Fachgebieten: }\end{array}$} \\
\hline & & $-\infty$ as $=-\ldots-\infty-\infty$ & -20 & 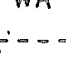 & $=-$. & $-\infty$ & $-\infty$ & $-\infty$ & $-\infty$ & $-\infty$ & $-\infty$ & $-\infty$ & $-\infty$ & $-\infty$ & $-\infty=$ \\
\hline$B W$ & 148 & $41-52$ & 11 & 21 & 5 & 6 & 1 & & 27 & 18 & & 3 & 2 & 6 & 10 \\
\hline BAY & 41 & 38 & 3 & 4 & 1 & 5 & 2 & 7 & 9 & 1 & & 6 & & 2 & 1 \\
\hline$B L N$ & 18 & 16 & 5 & 6 & 1 & 1 & & 2 & 3 & & & & & & \\
\hline BRE & 12 & 12 & & 8 & & & & & & & & 2 & & 1 & 1 \\
\hline HBG & 31 & 23 & 10 & 5 & 2 & 3 & 1 & 1 & 2 & 3 & 1 & 1 & & & 2 \\
\hline HESS & 21 & 21 & & 15 & 2 & & & & & & & & & $\hat{\imath}$ & \\
\hline NDS & 16 & 16 & 2 & 9 & & 2 & & 2 & & & & 1 & & & \\
\hline$N W$ & 35 & $15-34$ & 7 & 18 & 1 & & & & 4 & & & 1 & & & 4 \\
\hline RPF & 15 & $13-95$ & 4 & 3 & & & & & 5 & $\hat{1}$ & & 9 & & & 1 \\
\hline SAAR & 4 & 4 & & 2 & & $\hat{\imath}$ & & & 1 & & & & & & \\
\hline $\mathrm{SCHH}$ & 5 & 5 & 1 & 1 & & & 2 & & & & & 1 & & & \\
\hline UBA & 14 & 13 & 5 & 1 & & 1 & 1 & 1 & 1 & & & & & 3 & 1 \\
\hline$-\cdots-$ & $\cdots-\cdots$ & $-----\infty-\infty-\infty$ & --- & $-\infty$ & $-\infty-\infty$ & $-\infty$ & $-\infty-$ & $-\infty-\infty$ & $=-\infty$ & $-\infty-$ & . & $-\infty-$ & $-\infty$ & $-\infty$ & $-\infty$ \\
\hline & 360 & $216-268 *$ & 52 & 93 & 10 & 21 & 7 & 13 & 52 & 23 & 1 & 16 & 2 & 13 & 20 \\
\hline
\end{tabular}

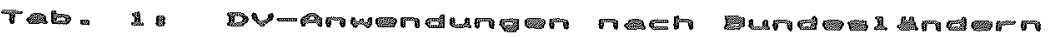

Bei der Interpretation der Zahlenangaben ist jedoch darauf zu achten, daß die auf den einzelnen. Fragebogen gemeldeten Computeranwendungen sehr verschieden umfangreich sind und von einfachen Modelirechenprogrammen bis hin zu großen Informationssystemen mit zahlreichen Teilsystemen reichen. Dias kann in einer derartigen statistik nicht entsprechend zum Ausdruck kommen. 


\subsection{Svetentechnische Asgekte des umwel tbezogenen Computereinsatzes}

Bei den in den Umweltbehörden eingesetzten Rechnerklassen überwiegen nach wie vor die Großrechner, der PC-Einsatz nimmt jedoch zu (siehe Abb. 3).

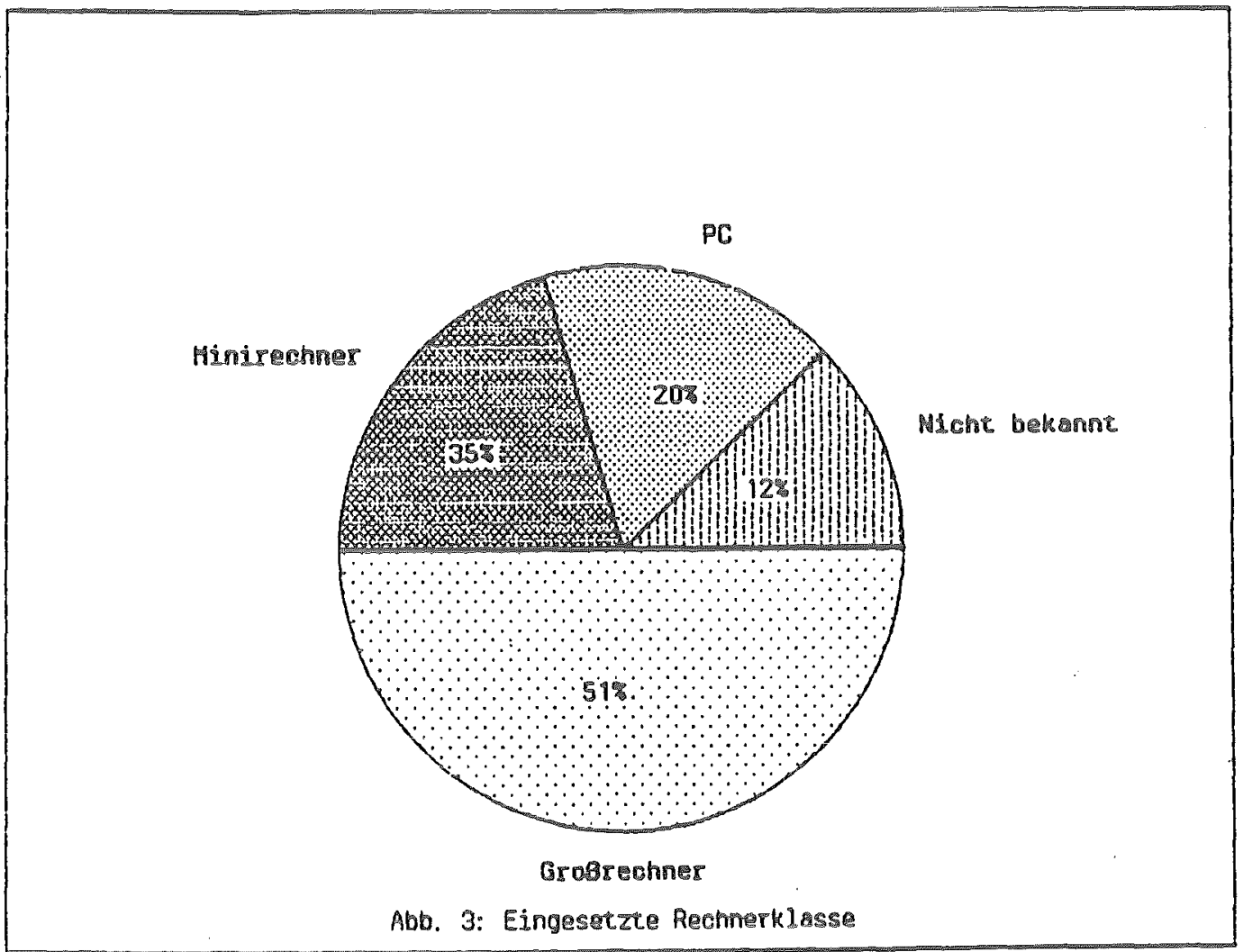


Hinsichtlich der bei der Realisierung von umweltbazoganen Computeranwendungen eingesetzten Programmiersprachen Fält die Dominanz von FORTRAN und das geringe Gewicht modernerer Sprachen WIE PASCAL aUF (ABb. 4 ):

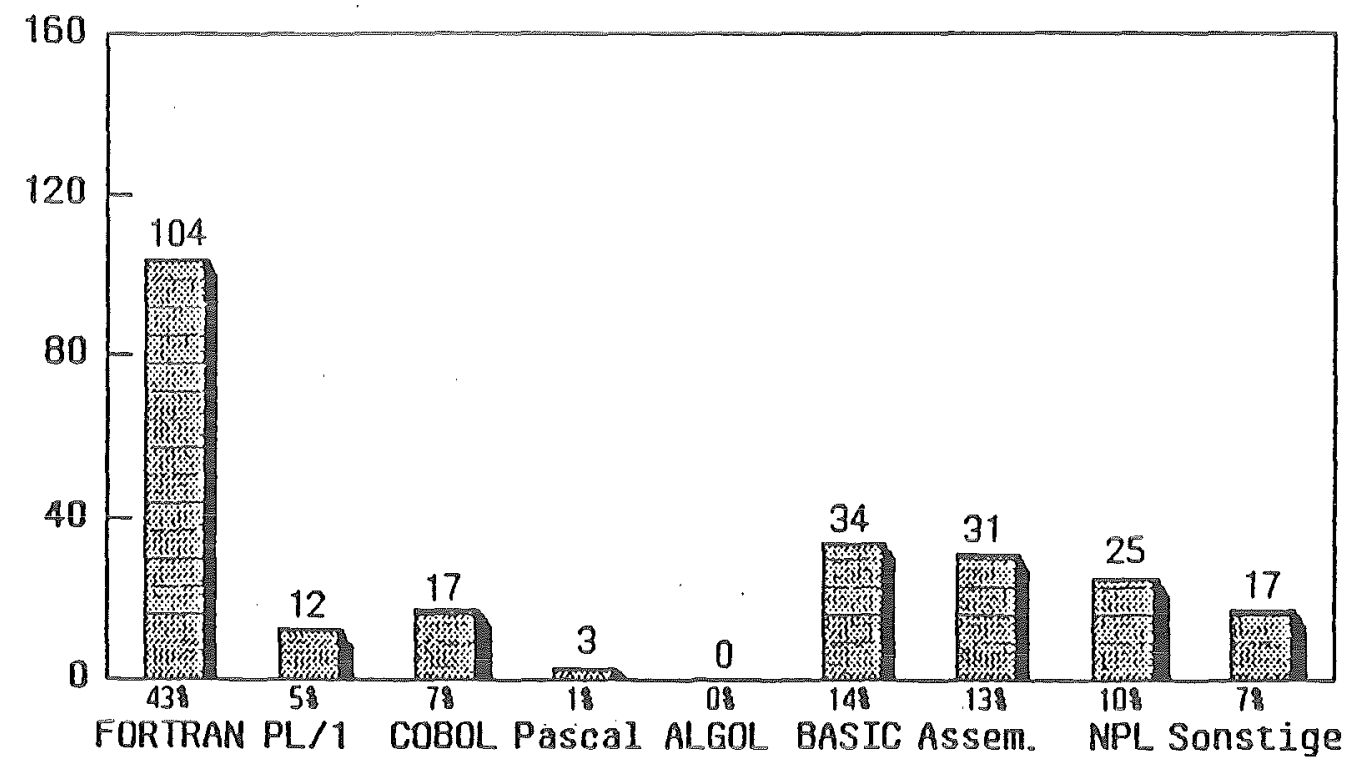

Abb. 4: Eingesetzte Programmiersprachen 
Bei der Benutzeranzahl je DU-Uerfahren ist bemerkenswert, daß der größte Teil der Computeranwendungen (45\%) über einen relativ kleinen Benutzerkreis von maximal 5 regelmäßigen Benutzern verfügt, davon wiederum ein Drittel über nur einen regelmäßigen Benutzer (Abb. 5). Eine mägliche Ursache für die geringen Benutzerzahlen kunnte in einer noch ungenügender BenutzerFreundichkeit der umweltbezogenen aU-VerEahren liegen.

$2-5$

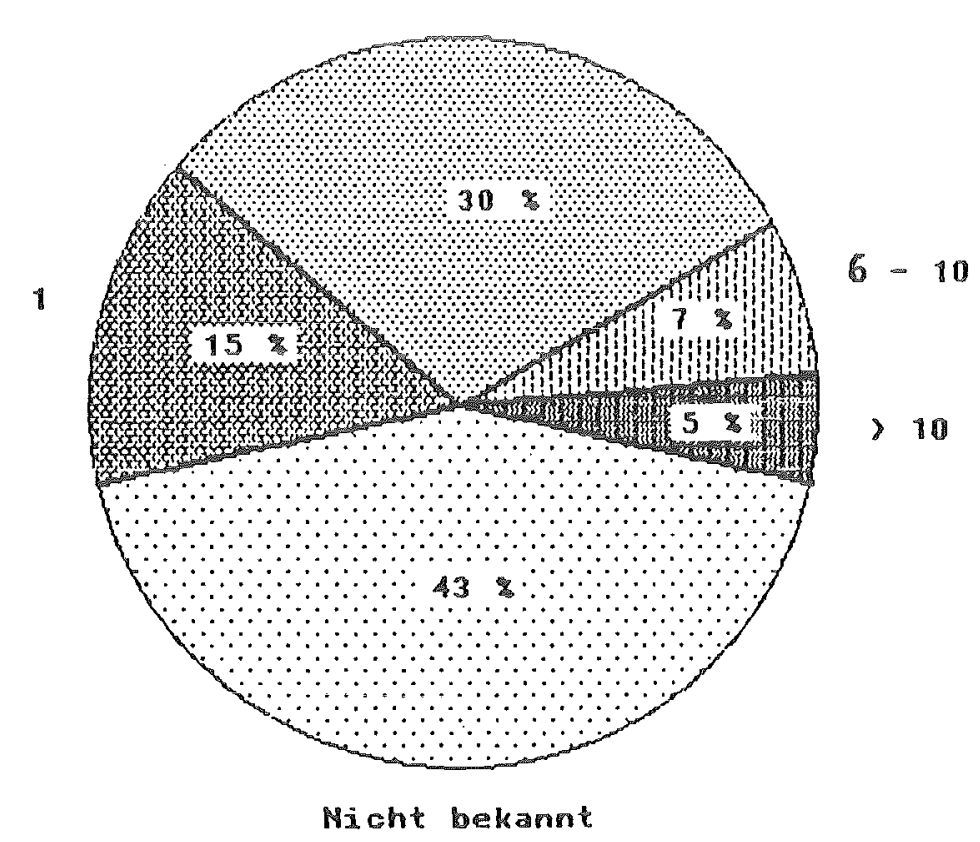

Abb. 5: Anzahl regelmäßiger Benutzer 
Dia Folgenden systemtechnischen Entwicklungstrande des umueltbezogenen Computereinsatzes können aus dem Umerageergebnis abgeleitet werden:

Datenbankeinsatz: Datenbanksysteme setzen sich zunehmend auch bai der Informationssystementwicklung auf dem Umweltsektor durch, insbesondere seit der kommerziellen Verfügbarkeit interaktiver Datenbanksprachen (wie z.B. NATURAL Für ADABAS-Datenbanken mit einer großen Verbreitung in den Umweltbehörden). Dia LaufFahigkeit gängiger Datenbanksysteme auch auF PC's solite zL einer weiteran Zunahme des Datenbankeinsatzes in den Umueltbehörden führen.

PC-Einsatz und Dezentralisierung: Das günstige Preis-/Leistungsverhältnis bei Mikracomputern Führt verstärkt zur PC-Anwendung in den Fachabteilungen. Dadurch gelingt es, die Hemmschwelle (auch zum Graßrechner) bei den Mitarbeitern zu senken, wodurch der Computereinsatz in den Umueltbehörden weiter gefördert wird. Erste Ansätze vernetzter Betriebsweisen (entweder als PC-Ringnetz oder in sternformiger Verbindung zum Großrechner) sind auf dem Umweltsektor zu verzeichnen. Das Problem von Zugriffsberechtigung und Konsistenz bei den zentralen Datenbeständen bedarf jedoch noch eines (auch organisatorischen) Lösungskonzeptes.

Graphische Datenverarbeltung: Für den Umweltbereich hat die Graphische Datenverarbeitung eine hohe Bedeutung. Bildliche Darstellungen umweltrelevanter Informationen sind für analytische Zwecke (Erkennen von Entwicklungen durch den Fachplaner), Für argumentative Zwecke (Unterstützung der EntscheidungsFindung und der politischen Durchsetzbarkeit von Umweltschutzmaßnahmen) und Für dokumentatorische Zwecke (Berichte, Broschüren) eine graße Hilfe. Dem Aspekt der Normung der graphischen Systeme ( $\mathbb{Z} . B$. GKS) zur Unterstützung des Austausches umweltrelevanter graphischer Informationen zwischen Umweltbehörden ist bisher nicht ausreichend Beachtung geschenkt worden. 


\subsection{Bewertung der DV-Umfrage in den Unweltbehörden}

Für die mit Umweltauggaben befaßten stabtichen Bahörden ist der Computer zum unverzichtbaran Hilfsmittel geworden. Der Realisierungsstand des Computereinsatzes ist jedoch in den einzelnen Umweltbehórden sehr unterschiedlich. Es reicht von vereinzelten PC-Programmen in den Fachabteilungen über umfangreiche Anwendungssoftwaresysteme für einzelne Umweltproblembereiche, die von graßen zentralen DU-Abteilungen selbst entwickelt und betrieben werden, bis hin zu gesamthaften Umweltinformationssystemansätzen ( $2 . B$. UMPLIS, siehe /PASBG/) bzw. behördenübergreifenden Systemkonzepten für umEassende landesweite UmweltinEormationssysteme (z.B. UIS Baden-Württemberg, $s$. /LANBE/).

Die langjährige organisatorische Zersplitterung der einzelnen Fachbehörden mit umweltbezogenen AuEgaben in sehr unterschiedlichen ministeriellen Ressorts hat dazu geführt, daß die meisten Computeraruendungen als isolierte Verfahran Eür Einzelaufgaben (Inselläsungen) realisiert worden sind. Dir systemtechnische und inhaltliche (datenmäßige) Kompatibilitat der Verfahren untareinander ist häufig nicht gegeben, dia Zusammenfassung innerhalb aines Gesamtkonzeptes daher problematisch.

Nicht nur im Wasserwesen, in dem der EDU-Einsatz bereits über eine lange Tradition (mehr als 30 Jahre) verfügt und das auch bis heute der wichtigste Anwendungsbereich vor der Luftreinhaltung geblieben ist, hat man schon Erühzeitig mit "Datenfriedhöen" $Z u$ kämpfen gehabt. Obwohl nach der vorliegenden Erhebung in jüngerer Zeit zahlreiche Auswertungsprogramme entwickelt wurden, erscheint dem Autor die Auswertungsseite nach wil vor unzureichend ausgebaut. Häufig werden nur die gesetzlichen Mindestanforderungen Für das Berichtswesen erfülit (z.B. Berichte zur Luftreinhaltung).

Die Anforderungen an den Computereinsatz nehmen auch in den Umuseltbehörden ständig zu. Der relativ hohe Anteil der geplanten Computeranwendungen im UmFrageergebnis ist ein Beleg dafür. Mit dem Anstieg des PC-Einsatzes werden auch in den Kommunen zunehmend umweltbezogene DU-Aktivitäten begonnen. Jedoch ist für den weiteren Ausbau der Computeranwendungen in den Umweltbehbrden die Entwicklung langfristiger umfassender InformationssystemGesamtkonzepte gefragt. Hier sind noch erhebliche Anstrengungen für die zukunft erforderlich. 


\section{For gchungsperspektiven für die Anquwandte infornatik in} Anwendungsbered ch Umwel t

In diesem Abschnitt werden einige Forschungsthematiken diskutiert, dil aus der Sicht des Autors sich Für die Angewandte Informatik auf dem Umweltsektor anbieten.

\subsection{Literaturdokumentation "Umwelt-Informatik"}

Der Informationssuchende mit Umweltinteressen ist nur schwer in der Lage, die Uielfalt und Menge der einschlägigen Fachliteratur zu überblicken. Daher werden seit längerer Zeit Literaturstellen in Literaturdatenbanken (mit Titel, Uerfasser, Abstract, etc.) abgespeichert und künnen im online-Retrieval über Deskriptoren zugegriffen werden (z.B. die Umweltliteraturdatenbank ULIDAT des Umweltbundesamtes). Auf besandere Schwierigkaiten wird der Interessent an Literatur über Informatikanwendungen auf dem Umweltsektor stoßen, da die Publikationen in sehr unterschiedlichen, bisher nicht unbedingt einschlëgigen Duellen (keine eigenen Publikationsargane) erscheinen. Ebenso Eehlt bisher ein aigenständiger Fachlicher Bereich mit ainer systematischen ErFassung der umweltrelevanten Publikationen mit Informatikbezug im Rahmen der Umweltilteraturdatenbank ULIDAI. Zwar konnten bei einer Literaturrecherche in der ULIDAT ca. 500 Quelien mit einem gewissen Informatikbezug nachgewiesen werden; ihre Deskribierung erfolgte jedoch nicht unter Informatikaspekten, zumal im ULIDAT-Thesaurus nur wenige Informatikbegriffe zur Verfügung stehen.

Als Basis jeder soliden Forschungsarbeit in einem Fachgebiet ist die systematische und kontinuierliche Auswertung der relevanten Literatur unabdingbare Uoraussetzung. Daher planen wir, in Zusammenarbeit mit dem Umweltbundesamt ein eigenstandiges fachliches Gebiet "Umwelt-Informatik" für die Umweltliteraturdatenbank aufzubauen und den Umwelthesaurus um wichtige Informatikbegriffe zu erwaitern. Erste Vorarbeiten für eine (vorerst lokal) computergestütze Literaturdokumentation "UmweltInformatik" sind in unserem Arbeitsbereich bereits angelaufen.

\subsection{Nicht-konventionel le Datenbankanwendungen}

Datenbanken und Informationssysteme gehören zu den grundlegenden Konzepten der Informatik Für die Informationsverarbeitung auF dem Umweltsektor. Datenbanken übernehmen die Funktion von Archiven in Informationssystemen. Sie bilden Pool mit allen interessierenden Daten eines Anwendungsgebietes, die dadurch zentral und integriert verwalket werden. Herkümmliche Datenbanksysteme orientieren sich heutzutage Fast ausschliaßlich an den klassischen ader "Standard"Datenbankanwendungen, wie sie im betriebswirtschaftlichen und administrativen Bereich vorherrschend sind. In den letzten Jahren verstärkte sich der Einsatz "nicht-kammerzililer" Rechneranwendungen, die auf dem Gebiet der Datenbanken als Nonstandard- (bzw. Nicht-konventionelle) Datenbankanwendungen bezeichnet werden (vgl. /HäRB5/). 
Zu diesen nicht-kanventionellen Anwendungsbereichen von Datenbanksystemen gehören U.a. Wissenschaftliche Auswertungen (Versuchs- und Meßdaten), Textverarbeitung und DokumentenRetrieval, Prozeßdatenverwaltung, Landinformationssysteme (Geographische Datenverwaltung) oder CAD- bzw. Graphische Datenverwaltung, die auch für Umweltanwendungen van Bedeutung sind. Um angepaßte Konzepte, Architekturen und Schnittstellen für Nicht-konventianelle Datenbankanuendungen entwickeln zu können, müssen genügend Kenntnisse über diese Anuendungen mit ihren Datenstrukturen und Dperationen, deren Typen, Verteilungen und Mengengerüsten erworben werden (/HäR85/). Aussagen aus der Literatur sind häufig zu unspezifisch, so daß PrototypEntwicklungen und Front-End-Ansatze auf der Basis existierender Datenbanksysteme ( $v g 1$. /APPBS/) herangezogen werden müssen, um dieses Wissen über die Anwendungsgebiete $z u$ ergänzen und zu konkretisieren.

Im Rahmen einer Diplomarbeit wird bei uns in zusammenarbeit mit der Hamburger Umweltbehörde ein Auswertungssystem für das Hamburger Luftmeßnetz auf Basis des Datenbanksystems SIR (hierarchisches System insbesondere für statistische Daten) und des statistischen Analysesystems SAS (mit Methodenbankarchitektur, s.u.) experimentell realisiert.

\subsection{Methoden-und Model 1 banksysteme}

Auswertungsmethoden (z.B. Für Wasser- oder Luftmeßdaten) und mathematische Modelie (z.B. Ausbreitungsmodelle) besitzen eine lange Tradition und eine hohe Bedeutung auf dem Umweltsektor (bis hin zum Normcharakter von Modellen in gesetzlichen Regelungen). Die in diesem Bereich eingesetzte Softwaretechnik ist jedoch aus der Sicht der Informatik als nicht adequat zu bezeichnen. Sie geht häufig nicht über individuelle Programme in wenig strukturierten Sprachen wie FORTRAN oder DYNAMO (Simulationssprache für kontinuierliche Systeme) hinaus. Die Angewandte Informatik kann jedoch Softwarekonzepte anbieten, die den hohen Anforderungen an die Flexibilitat und Benutzerfreundichkeit bei der Anwendung von Methoden und Modelien im Umweltbereich wesentlich besser gerecht werden. Dazu gehören die Methodenbank- und Modelibanksysteme, die zwar als. Forschungsthematik der Angewandten Informatik nicht mehr über die Aktualität der vergangenen Jahre verfügen, jedoch vom Autor nach wie vor als relevante Konzepte Für bestimmte Anwendungen auf dem Umweltsektor (Auswertungssysteme, Interaktiver Betrieb von Umueltmodellen aus einer Modellbasis) angesehen werden.

Unter einem Methoden-/Modellbanksystem ist eine erweiterbare Sammlung von Programmbausteinen $z u$ verstehen, die Methoden und Modelle repräsentieren - unter der zentralen Verwaltung mit einem benutzer- und problemorientierten Zugriff (/PAGB3/).

Im Umweltbundesamt sind Anfang der achtziger Jahre im Rahmen eines BMFT-Projektes bestimmte Grundfunktionen eines Methodenbanksystems, insbesondere Eür die Verwaltung graphischer Methoden, entwickelt worden /FIHBO/. Methodenbankaspekte sind auch in dem 
oben erwähnten, bei uns entwickelten experimentellen Auswertungssystem Für die Hamburger LuFtmeßdaten von Bedeutung. Die Anuendung von Modelibanksystemen (hier des MBS der GMD) Für den Betrieb von Umweltmodellen wird in /HIPBGb/ diskutiert.

\subsection{Intrektive Mode11 ierungs- bzw. Simulationssvetene}

Wahrend bei Modellbanksystemen die Verwaltung und der Betrieb bereits realisierter ( Teil-) modelle im Vordergrund steht, geht es bei Modelilierungs- und Simulationssystemen vor allem um die Computerunterstützung des gegamten Prozerseses der Modellbildung und Simulationg von dor Planung, dem Aurbau, und der Dokumentation der Modelde bis zuF grahpiechen Autbereitung. Durch einen hohen Grad an Interaktivitat in allen Systembereichen kann dem Benutzer die interaktive Modeld erstel Iung direkt am Bildschirm, dia interaktive Steuerung des simulationsabiaures sowie die interaktive Aufbereitung der sinulationsergebnisge angeboten werden. 2 iel ist oin hohes Maß an Benutzerfreundichkeit bei der computerunterstützten Modellierung auch für Nicht-DU-Spezialisten, die bei der systemanalytischen Modelibildung $(z . B$ auch von akasystemen) als Benutzertyp dominierend sind.

In unserem Arbaitsberaich ist als einer der Farschungsschwerpunkte ein derartiges interaktives Modellierungswerkzeug, das System DYNAMIS, entwickelt worden, das die interaktive Erstellung und Ablau von Systems Dynamics-Modellen cvor allem auch des ökologischen Bereichs) unter Nutzung neuartiger (objektorientierter) Benutzerschnittstellen auf einem leistungsfahigen Mikrocomputer mit besonderen grahischen Funktionen (Apple LISA/MacIntosh) ermöglicht. Dazu wurde eine modifizierte Uersion der Sprache DYNAMO (in PASCAL) implementiert, die U.a. Sprachkonstrukte für einen modularen Modellaufbau aus Teilmodelien anbietet.

Die Modelilerung im System DYNaMis wird durch dessen abjektorientierte Benutzeraberflache insbesondere für den nicht computererfahrenen Simulationsanwender sehr vereinfacht. Die wichtigsten objekte auf der obersten Ebene stellen die Modelle dar, die als Einheit bearbeitet werden können (Kopieren, Lüschen, etc.). Ein Modell besteht wiederum aus objekten (der niedrigeren Ebene) wie "Modell-Beschreibung", "System Dynamics-Diagramm", "(Simulations-) Programm", "Bezeichnerliste (der Model Ivariablen)" und "Ergebnisübersicht".

Bei der Durchführung der Simulationsexperimente erfolgt eine begleitende graphische Ausgabe, die den Werteverlauf ausgewählter Modellgräßen während eines Simulationslaufes anzeigt. Dadurch kann der Benutzer gezielt in einen Simulationslauf eingreifen und den Madelizustand verändern. Die steuerung des dynamischen Systemablaufs liegt in der Hand des Benutzers (z.8. beliebiger Wechsel der bearbeiteten bbjekte oder Unterbrechnung bzw. Rücksetzung der Simulation. Zur Auswahl und zum Starten der auszuführenden Aktionen wird zur Benutzerunterstützung bing graphikorientierte Menütechnik eingesetzt wird. 
Die Folgende Abbildung 6 vermittelt einen Eindruck von der interaktiven Arbeit mit dem System DYNAMIS aus Benutzersicht. Die einzelnen Bereiche des Madelis können mit HilFe der Fenstertechnik auf dem Bildschirm dargestelit werden. Man sieht hier einen Bildschirm mit Ausschnitten des SD-Diagramms und des DYNAMDPogramms eines (ökologischen See-) Modelis (übereinander gelegt). Die graphische Modellierung (Erstellung des SD-Diagramms) erfolgt durch Selektion der entsprechenden Symbole aus dem Menu am 1 inken Rand des Diagrammfensters und Pasitionieren des Cursors auf dem Bildschirm (Dimensionierung über Zoomeunktion) mit Hilfe der Maus (als Inputdevice). Die beiden Dbjekte "Diagramm" (aktiviert) und "Programm" werden durch das auFgeklappte Menü der Funktionslaista "Bearbeitung" mit den ausuälbaren Aktionen in diesem Systamzustand überlagert. Die Funktionsauswahl erfolgt wiedarum durch Positionieren des Cursors und Anklicken mit Hilfe der Maus.

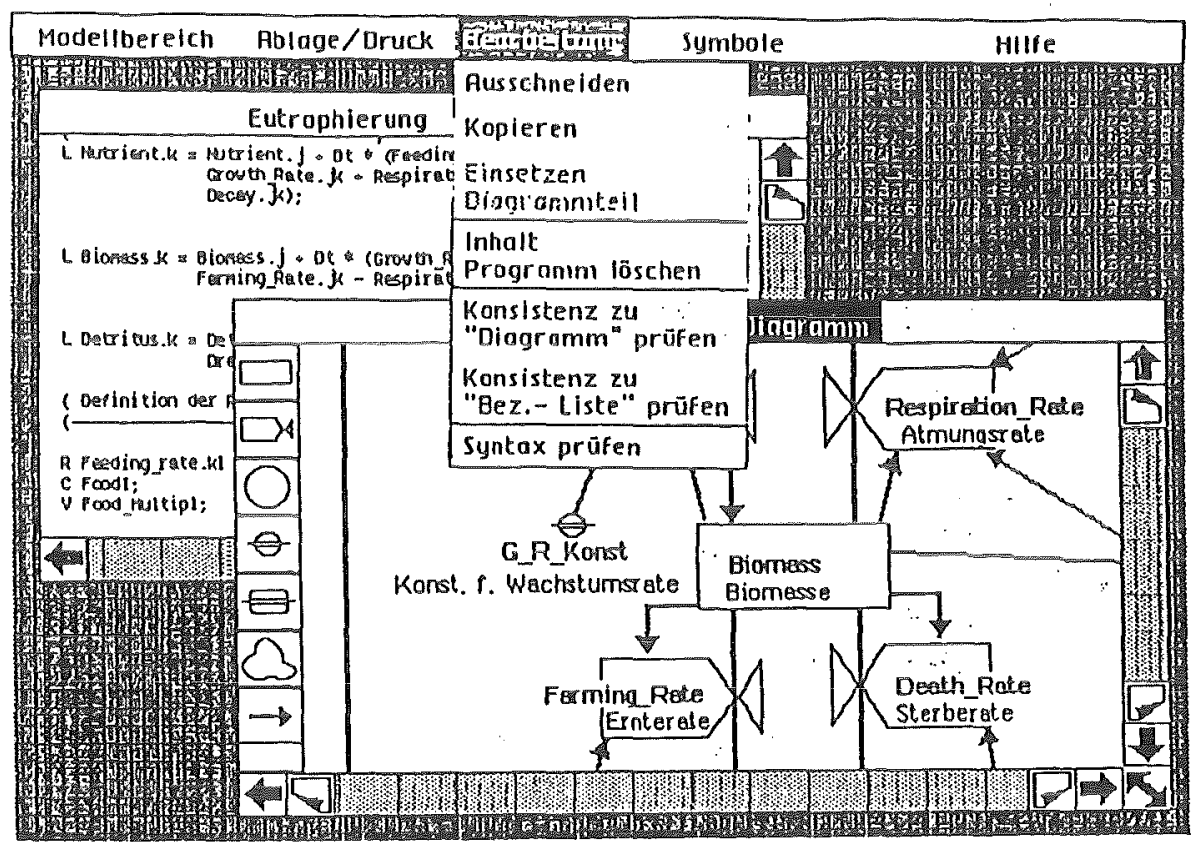

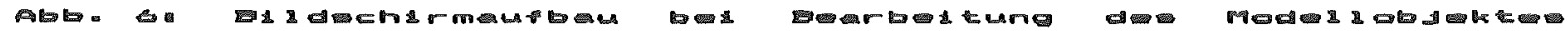

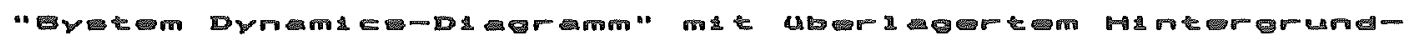
Pै
}

Das Gesamtkonzept und die Implementierung des Systems DYNAMIS sind in /BHPBG/ ausführlicher dokumentiert. Eine Anwendung von DYNAMIS in der ökologischen Systemmodellierung (Simulation der Eutrophierung eines Sees] ist in /HNSB6/ beschrieben. 
Konzepte Interaktiver Modelilerungs- und Simulationssysteme gewinnen einerseits in der Forschung der verschiedensten wissenschaftlichen Disziplinen (auch für die b̈kologische Systemmodeliferung) immer mehr an Bedeutung. Andererseits können sie auch im Rahmen von sog. Decision Support Systems (DSS) Für die (umwelt-) politische Entscheidungsvorbereitung eingesetzt werden, wenngleich diese Anwendung bisher wohl eher zu den Ausnahmen zu zählen ist.

Allgemein sollen DSS Führungskräften und ihren entscheidungsvorbereitenden Stabsstelien im.Computerdialog Entscheidungshile in in Form von problem- oder problemklassenbezogenen und modellbasierten Lüsungen anbieten. Typische Methoden und Werkzeuge sind Verfahren der statistik (Deskriptiv, Prognose, etc.) und des Dperations Research (Simulation, Dptimierung, etc.), jedoch auch spreadsheet-orientierte Planungssprachen. Zunehmend gewinnen auch Methodiken der Expertensysteme an Bedeutung Eür das Gebiet der DSS.

Ein DSS zur computergestützten Bereitstellung von Entscheidungshilfen für die UmweltverträglichkeitsprüFung, das amerikanische Environmental Technical Information System, ist in /PAGBgc/ beschrieben. ETIS bietet dem Benutzer in vielerlei Hinsicht "Expertenwissen" an, wenngleich auch nicht in den für heutige Expertensysteme typischen wissensrepräsentationsformen c vgl. /SCHBE/). Hier soll jedoch beispielhaft ein DSS für die Entscheidungsvorbereitung bei umweltrelevanten Planungan angerissen werden, das am Zentralen Institut für Kybernetik und Informationsprazesse der Akademie der Wissenschaften der DDR entwickelt wurde und in verschiedenen ländern in der Palitikberatung eingesetzt wird. Grundlage Eür die Darstellung ist /SYDBE/.

Im Rahmen von lang-, mittel- und kurzeristigen

Im Rahmen von lang-, mittel- und kurzeristigen Planungsaufgaben Entscheidungen über Art, Umfang und Abfolge von

- Investitionen und Maßnahīen im Umweltschutz

- Schutzmaßnahmen für die wälder und landwirtschaftiche Produktion

- Einführung technischer Innovationen in den Produktionsprozeß

getroffen werden, um den Schadstoffausstoß der Emittenten und deren Folgen zu verringern. In Anbetracht des hohen Komplexitatsgrades der Planungsprobleme bieten sich systemanalytisch basierte Entscheidungsmodelle als Entscheidungshilfen an. In dem vorliegenden DSS sind 3 hoch aggregierte Submodule enthalten, und zwar

(1) ein Produktions-/Emissionsmodell u.a mit technologischen Daten und Produktionsmengen als Inputgráßen und dem Verlauf der räumlichen Schadstoffemisianen in LuFt und Wasser als Model1output 
(2) ein Transmissionsmodell für Schadstoffemittenten/Immissionen in Luft und Wasser mit metereologischen Angaben und berechneten Emissionen aus Submodell (1) als Inputparametar sowil dem räumlichen Uerlauf der Immissionskonzentration als Dutput

(3) ein Modell Eür die Immissionswirkungen in Wald-, Wasser-, landwirtschaftlichen bkosystemen mit Inputparametern über das analysierte bkosystem, aus metereologischen Verläufen und in Form von berechneten Immissionskonzentrationen aus Submodel 1 (2). Modelloutput sind ökasystemspezifische Indikatorzustandsvariablen mit deren Bewertungsfunktionen.

Mit dem Modell lassen sich Szenarien entwickeln Eür die Abschätzung der Auswirkungen spezieller Investitionsalternativen zur Emissionsminderung in Produktionsanlagen bzw. Für Schutzmaßnahmen (Düngung) des Waldbestandes. Darüberhinaus ist ain Softwaremodul Eür die multidimensionale pptimierung in das osS integriert, um dem Entscheidungsträger, der sich im Umweltbereich typischerweise in einem mehrfachen Zielkonflikt befindet, eine rechnergestützte Entscheidungshilfe zur sukzessiven Auffindung einer ausgewogenen Kompromißlüsung anzubieten. Zur Zeit gehen 4 konkurrierende Zielfunktionen in die Bestimmung der aptimalen Kompromißlósung ain, wobei die subjektiven Präferenzen des Entscheidungsträgers berücksichtigt werden. Diese gegenläufigen Ziele sind die folgenden:

- Minimierung der Gesamtemissionen aller Emittenten einer betrachteten Region

- Maximierung der Anzahl der besonders relevanten Gebiete, in denen sich die geforderten Immissionsminderungen umsetzen lassen

- Minimierung der Gesamtinvestitionskosten

- Minimierung des Planungshorizontes.

Als Ergebnis dieses mehrdimensionalen Optimierungsverfahrens erhält der Benutzer die Immissionsverteilung zuischen allen Gebieten und für jedes Planungsjahr sowie den Verlauf des Investitionsbedarfs $z$.T. in graphischer Form. 
Die Systemstruktur des DSS ist in Abb. 7 grob dargestellt.

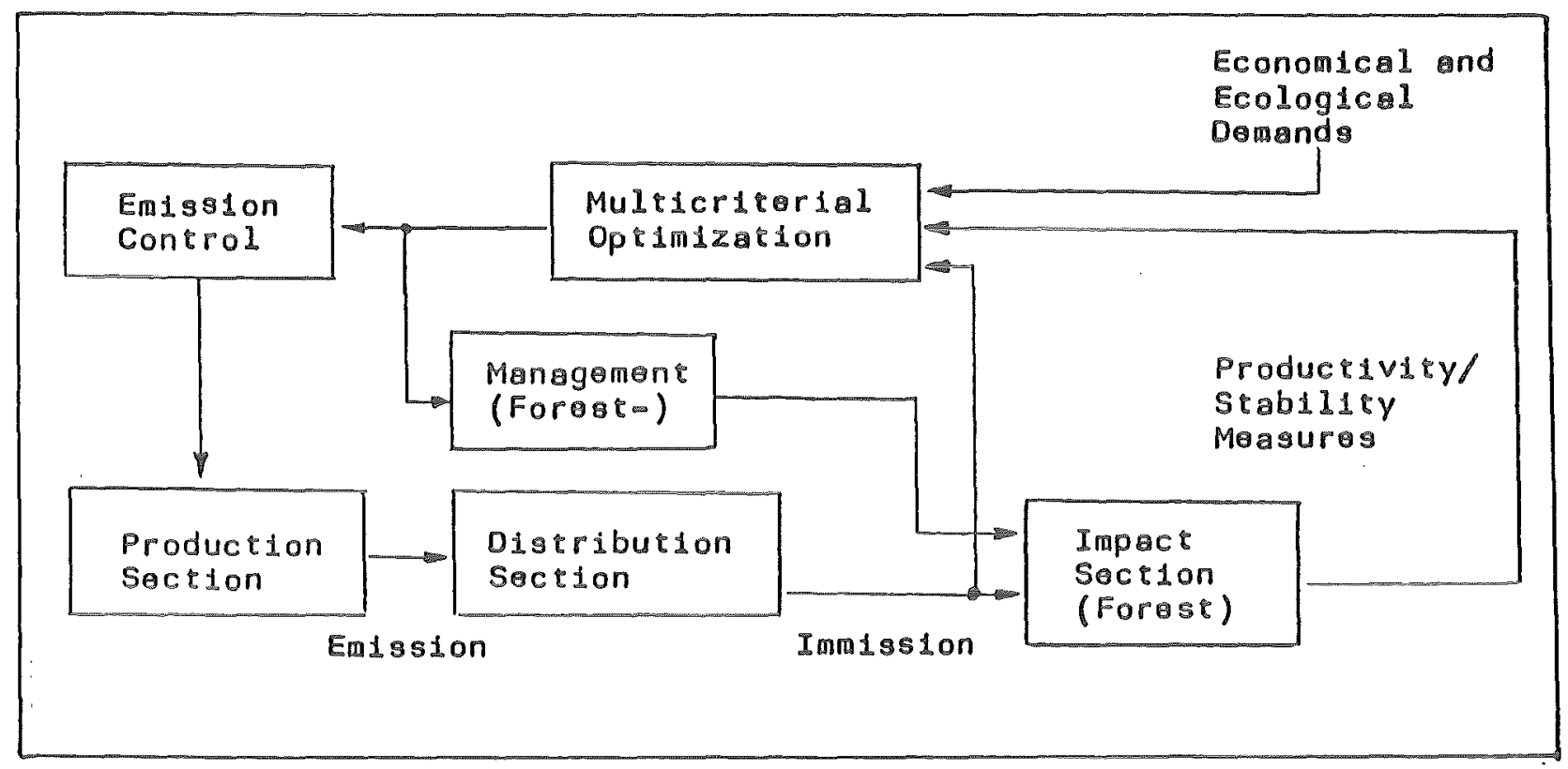

만 5

Brobarembers

물

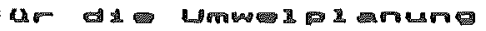

T)

\subsection{Expertengystme}

Unter den aktuellen Entwicklungen in der Informatik kammt zweifelsohne der Künstlichen Intelligenz - und hier insbesondere den Expertensystemen - eine besondere Bedeutung $z u$.

Unter einem Expertensystem ist ein wissensbasiertes System zu verstehen, welches das Wissen von qualifizierten Experten für ain bestimmtes eingegrenztes Gebiet modelliert und benutzt, um komplex日 Probleme eines bestimmten Iyps aus diesem Gebiet zu 1 bsen bzu. deren Lösung zu unterstützen.

Bei den Anwendungen van Expertensystemen lassen sich drei Hauptkategorien unterscheiden:

- Interpretation von Daten, Befunden, Symptomen, um daraus eine Diagnose bzw. eine Aktion vorzuschlagen ( $z . B$. Interpretation von Meßdaten oder bkologische Bestimmungsaufgaben)

- Unterstützung bei Entwurf und Planung in komplexen Situationen ( $z . B$. Umweltverträgliche Planung)

- Weitergabe von Wissen: Ausbildung, Training, Simulation (z.B. Uerhalten bei Störfällen). 
Auf dem Umweltsektor sind bisher kaum Expertensystem-Anwendungen bekannt geworden. In /Hang5/ beispielsweise wird über den Einsatz van Expertensystemen zur Abwasserbeseitigung berichtet, in /HäBG6/ über ein Expertensystem Umweltrecht. /SCHB6/ beFaßt sich mit den methodischen Grundlagen von Expertensystemen als Darstellungsmethodik Eür entscheidungsunterstützende Systeme im Umweltbereich. An dieser Stelle soll kurz ein Expertensystem zur Artenbestimnung im Naturschutz skizziert werden, das im Rahmen eines Projektseminars an unserem Fachbereich entwickelt wurde und in /HILES/ beschrieben ist.

Das Expertensystem ermüglicht - als prototypisches Beispiel einer -allgemeinen Bestimmungsaufgabe - die Identifikation von in Deutschland heimischen Libellen. Das Expertenwissen cdie Bestimmungsschlüssel) konnte dabei aus einem bialagischen Bestimmungsbuch entnommen werdan.

Ein Expertensystem zur abjektbestimmung ist verwandt mit den sag. Diagnosesystemen, zu denen auch das bekannte MYCIN-System gehört, in dem das Expertenuissen in Form von Produktionen (d.h. Regeln) abgespeichert ist. Im Gegensatz zU MYCIN, das als Lüsungsstrategie ein Backward-Chaining-WerEahren (ausgehend von einer Hypothesenmenge wird versucht, über Fragen an den Benutzer eine Hypothese zu verifizieren), eignet sich bei Bestimmungsaufgaben eher die Forward-Chaining-Methode (Suche der Lösung durch Fragen, ausgehend von den gegebenen Daten, d.h. dem Anfangszustand). Dann lassen sich die Fragen eines Bestimmungsbuches direkt in Regeln transformieren und die natürliche Systematik bleibt erhalten.

Als Implementierungssprache wurde die regelbasierte KI-Sprache DPSS eingesetzt. OPS5 verfügt über eine globale Datenbasis, den working Memory, welches aus einfach strukturierten Elementen mit Elementnamen und assoziierten Attribut-Wert-Paaren besteht, wie z.B. (vgl. /HIL86/, 5.429):

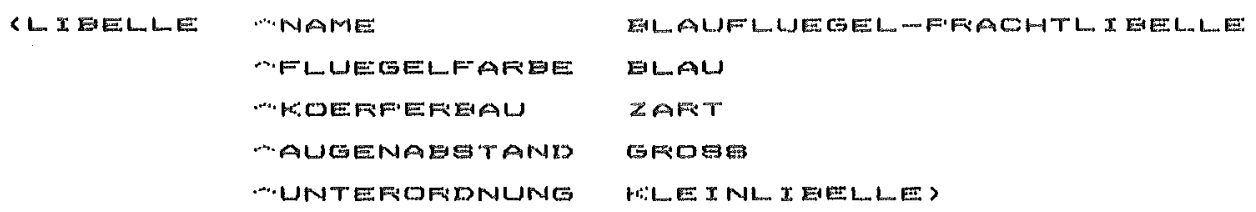

Im Bestimmungssystem ist bei jeder Frage die Antwort "unbekannt" zugelassen. Damit ist gewährleistet, daß immer neue Fragen aus dem Fragenvarrat gestelit werden. Außerdem können vom Benutzer wahrend des Bestimmungsdialoges jederzeit eine Backtracking-Funktion aufgerufen und bisher gegebene Antworten verändert werden. Werden durch die modifizierten Angaben bereits ausgeschlossene Libellenarten wieder zu mäglichen Bestimmungskandidaten, so werden sie mit ihren assoziierten Attributen reaktiviert. 
Abbildung $\theta$ zeigt einen Ausschnitt aus einem typischen (in geschweiften Klammern kommentierten) Benutzerdialog mit dem Expertensystems zur Libelienbestimmung (Eür den voliständigen Dialog, siehe /HIL86/, S.434-437). In diesem Beispiel war es mit Hilfe des Expertensystems in einem Dialog mit nur 12 Fragen möglich, die Libellenarten auf drei mägliche Kandidaten einzugrenzen, obwohl der Benutzer sechs Fragen nicht beantworten konnte.

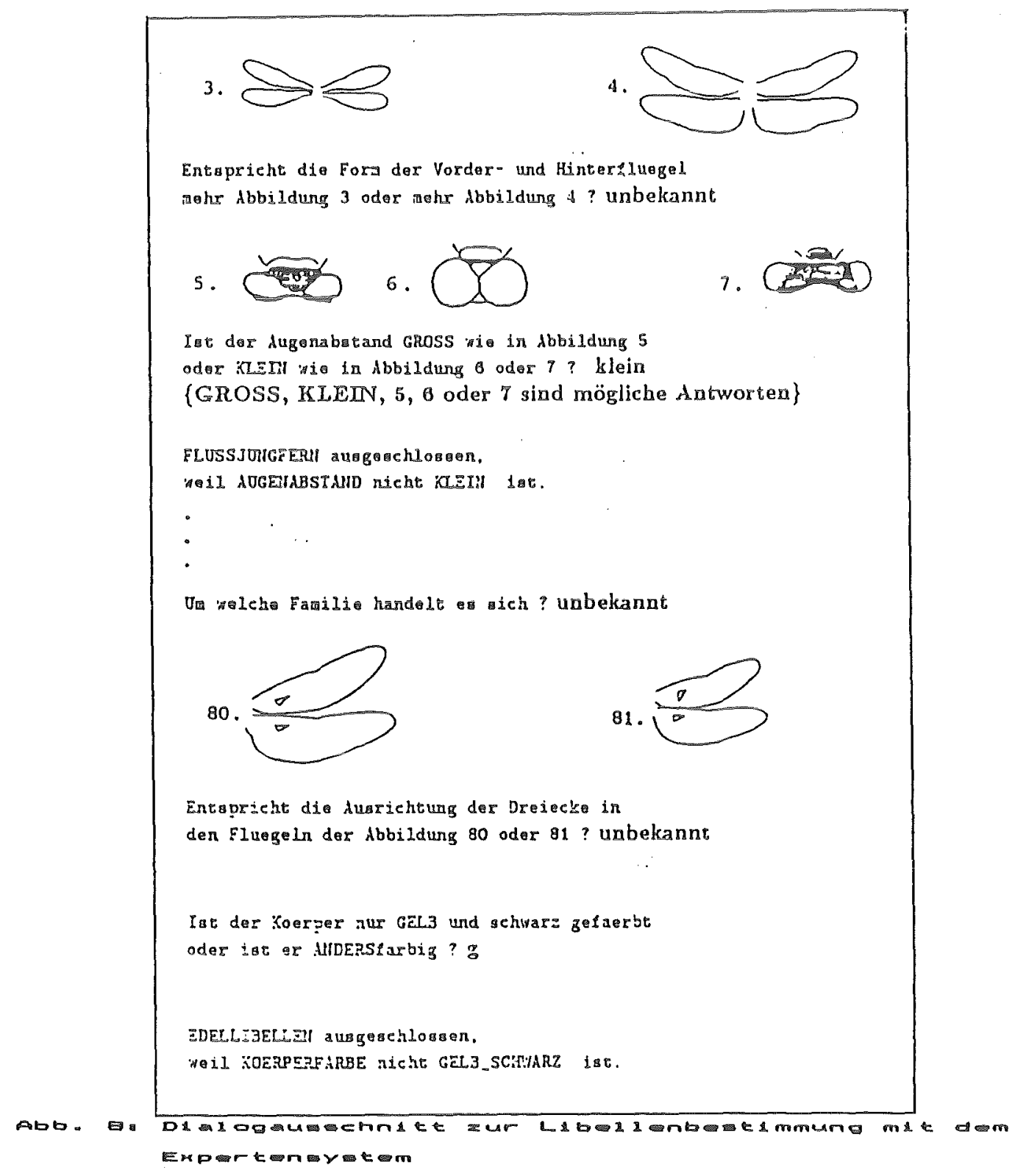

Im Gegensatz zur häufigen Einbettung des objektspezifischen Wissens in das Regelsystem wurden das Expertenwissen und die Texte Für den Frage-Antwort-Dialog auE externen Dateien abgespeichert. Nur die allgemeinen Regeln zur Fragenauswahl und zum Ausschluß einzelner Dbjekte sind im Produktionensystem enthalten. Damit ist das Expertensystem durch Austausch der Wissensbasis und der Bilddaten auch für die Bestimmung anderer (nicht nur äkologischer) objektklassen einsetzbar. 


\section{SchluBbetrachtung: Auf dem Wea zu einar "Umwelt-Informatik"?}

Die Bewältigung der vielseitigen, stark vernetzten und sehr interdisziplinären Aufgaben im Bereich des Umweltschutzes, der Umweltplanung und der Umweltforschung verlangt mehr denn je den verstarkten Einsatz moderner Konzepte und Methoden der Informationsverarbeitung. Der Computereinsatz kann in bestimmten Teildisziplinen des Umweltbereiches wie $z . B$. dem Wasserwesen auf eine lange Geschichte zurückblicken. Dennoch entspricht der Entuicklungsstand der Computeranwendungen in vielen Umweltbehörden (und auch in vielen Bereichen der Umweltforschung) aus der Sicht der Informatik bis heute noch nicht dem stand der Technik (vgl. Kap. 2). Insbesondere mangelt es in den Umweltbehórden an gesamthaften, umfassenden und integrierten Informationssystemansätzen; es existieren (auch) auf dem Umweltsektor zu viele Insel lósungen. Als wichtigste Zukunftsaufgabe stellt sich für die Umweltbehörden die Entwicklung und Umsetzung eines aufgabenorientierten, informationstechnischen (und organisatorischen) Rahmen-konzeptes Eür ein Umwelt-Informationssystem zur Bereitstellung von Umweltdaton als EntscheidungshilEen in den verschiedenen Umweltbereichen. Die Umsetzung dieser Zielvorstellung wird jedoch angesichts des jetzigen Realisierungsstandes der Computeranwendungen in der Umweltschutzpraxis und des hohen erforderlichen Aufwandes wahrscheinlich noch weit bis in die neunziger Jahre dauern.

Hierbei könnte die wissenschaftliche Disziplin der Angewandten Informatik wesentliche Hilfestellung leisten, wenn sie sich diesem anspruchsvolien und gesellschaftlich bedeutsamen Anwendungsgebiet im Sinne einer praxisorientierten Forschung verstarkt annämme. Einige sich anbietende Forschungsthematiken der umweltbezogenen Infarmatikanwendungen sind im Kapitel 3 dieses Beitrages diskutiert worden, weitere sind denkbar (z.B. Kopplung von dkologischen Expertensystemen mit Simulationsmodelien).

Jedoch kann nicht nur der Umweltschutz von einer verstarkten Forschungstatigkeit der Informatik auf diesem Anwendungsgebiet profitieren, sondern auch die Angewandte Informatik selbst. Denn der Umweltsektor bietet eine Fülle von Problemeleldern hoher Komplexität, Interdisziplinaritat und Vernetzungsgrad, die der Angewandten Informatik als anspruchsvolle Prüfsteine für die Methodenentwicklung und -kritik - im Sinne einer "Methodenvalidierung" - dienen künnen. Uon umweltbezogenen Informatikanwendungen lassen sich durchaus Impulse für die Weiterentwicklung von Informatikmethoden erwarten. 
Ein weiterer Worteil dieses Anwendungsgebietes läge für dia Informatik in der Chance, sich auch in die Gruppe derjenigen technisch-wissenschaftlichen Fachdisziplinen einzureihen, die ihre Forschungsaktivitäten verstäkt in den Dienst des Umweltschutzes stellen (wie Biologie, Chemie, Verfahrenstechnik, Regelungstechnik, U.a.j und ihren Teil zuL (technologischen) Bewältigung der Umweltkrise beizutragen. Damit könnte der vorherrschenden Skepsis gegenüber der Informatik allain als Rationalisiarungs- oder Rüstungs- (SDI-) technologie in graßen Teilen der bffentlichkeit durch Aufzeigen alternativer, gesellschaftlich allseits akzeptierter Anwendungsberaiche wie den Umweltschutz begegnet werden.

Schon jetzt eine neue eigenständige Fachdisziplin "UmweltInformatik" zu proklamieren, hält der Autor allerdings Eür verfrüht. Dazu ist die wissenschaftliche Basis noch $z u$ schwach ausgebildet; ein eigenständiges wissenschaftlich-methodisches Profil muß sich erst herauskristallisieren, zumal der Fachliche Gegenpol - die Umwelteorschung - sich ebenfalls noch nicht endgültig als eigenstandige Fachwissensehaft formiert hat. Anders ist dies bei der Medizin, die sich als eine klassische, voll etablierte Fachwissenschaft schon Frühzeitig der Methoden der Informatik auf wissenschaftlicher Basis bediente, woraus bereits vor mehr als 15 Jahren eine eigenständige Anwendungs-InEormatik die Medizinische Informatik - eruuchs (zeitlich ziemlich parallel zur Entwicklung der Kern-InEormatik). Daher war diese auch in der Lage, wesentliche Anstöße zur Methodenentwicklung in der Informatik beizusteuern ( $z \cdot B$. durch die Entwicklung medizinischer Expertensysteme). Wir haben es jedoch im Umweltbereich heute mit einer für die Bildung einer eigenen Anwendungs-Informatik von der wissenschaftlichen Seite her weniger günstigen Ausgangsposition zu tun als seinerzeit bei der Medizin; dies sollte bei einer zu frühzeitigen Anmeldung eigener wissenschaftlicher Ansprüche bedacht werden. Andererseits gewinnt der Umweltsektor als wirtschaftlicher und gesellschaftlicher Faktor rasant an Bedeutung mit immer neuen Anforderungen an eine moderne Informationsverarbeitung (s.a.), so daß die Angewandte Informatik von der Praxis aus verstärkt in dieser Anwendungsrichtung gefordert (und auch gefördert) werden könnte ( $2 . B$. einschlägige FuE-Vorhaben, neue Arbeitsschwerpunkte in den GroßEorschungseinrichtungen, etc.). 
Auch sollten die Uorbehalte der Informatik gegenüber einer drohenden Zersplitterung durch immer neue "BindestrichInformatiken" ("von der Archäologie-Informatik bis zur ZoologieInEormatik") als Gefahr für den wissenschaftlichen Standard der Informatik insgesamt durchaus ernst genommen werden. Mangelnde wissenschaftliche Fundierung würde den ohnehin starken Akzeptanzwiderständen von Seiten der chäufig nur wenig anwendungsinteressierten) Kern-Informatiker gegenüber den AnuendungsInformatiken nur weiteren Vorschub leisten. Die Schaffung oiner soliden. wissenschaftlich-methodischen Basis der umweltbezogenen Informatikanwendungen solite daher die Aufgabe der nächsten Jahre sein. Auf dieser Basis lassen sich dann eigene wissenschaftliche Ansprüche offensiv vertreten, denn methodische Grundlagenforschung in der Kern-InEormatik allein führt noch nicht automatisch zu Fortschritten in der anwendungsbezogenen Informationsverarbeitung. Die Anwendungs-Informatiken beziehen ihre Daseinsberechtigung doch daraus, daß sie für einen Methodentransfer in das betreffende Anwendungsgebiet sorgen, sie vermitteln das notwendige "Umsetzungswissen", ohne das es keine anspruchsvolle Informationsverarbeitung in den Anwendungsbereichen geben kann.

Wann dieser Weg uns von einer speziellen Forschungthematik "Anwendungen der Informatik im Umweltbereich" innerhalb der Informatik schließlich zu einem eigenstandigen Fachgebiet "UmuleltInformatk" (mit eigenen regelmäßigen Konferenzen, Publikationsorganen, wissenschaftlichen Arbeitsgruppen, etc.) Eührt jedoch auch weiterhin mit notwendigerweise engen Anbindungen zur Informatik - , hängt von den Arbeitsergebnissen der nächsten Jahre ab. Der Autor ist jedoch davon überzeugt, daß sich dieser weg lohnt, und zwar sowohl Eür den Umweltschutz als auch für die Angewandte Informatik! 


\section{Literatur}

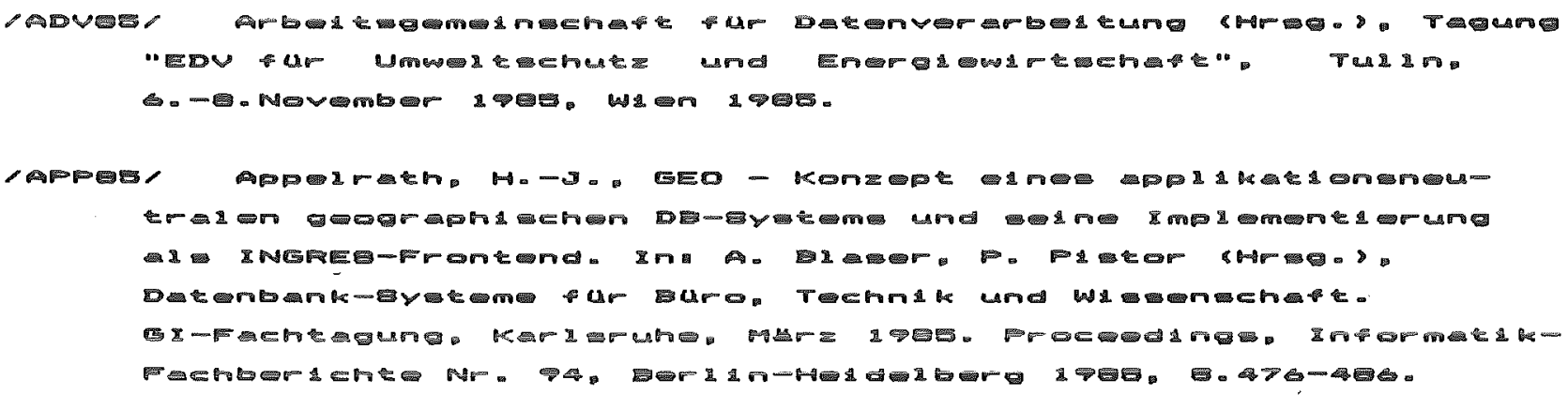




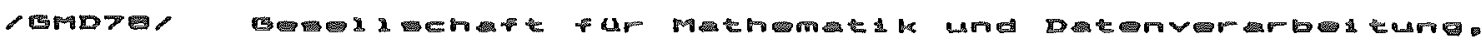

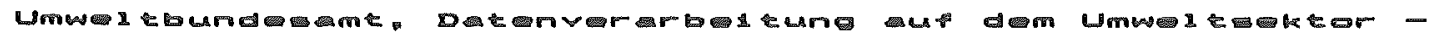

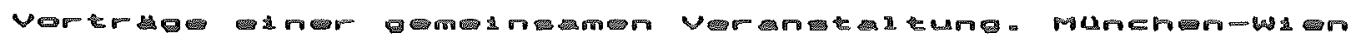
$19>9$

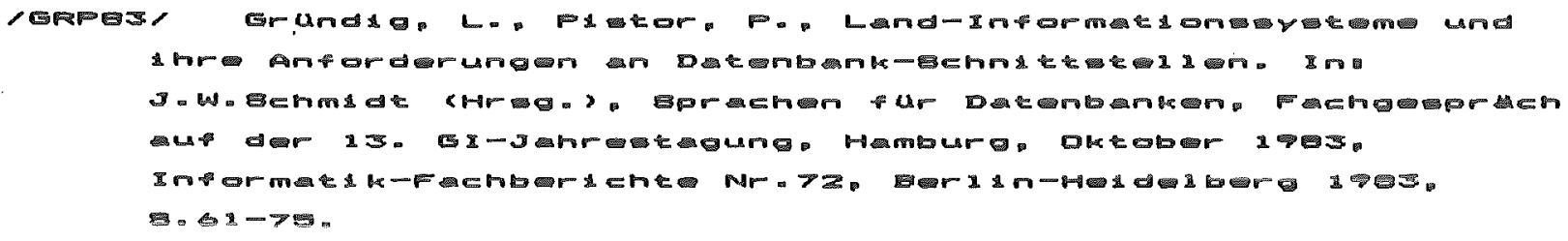

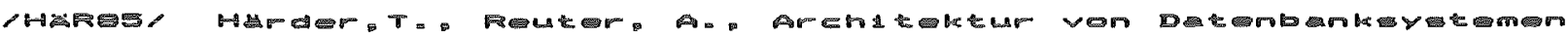

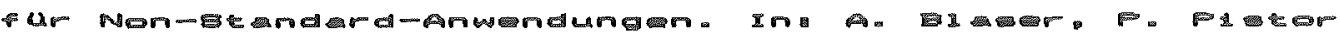

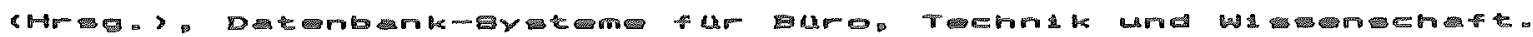

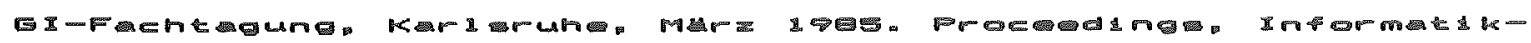

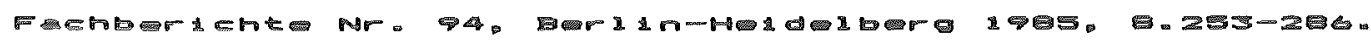

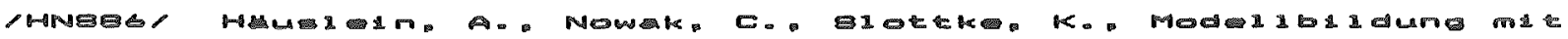

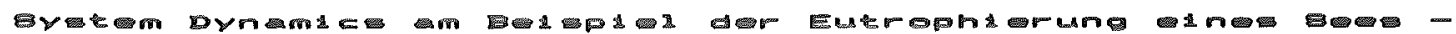

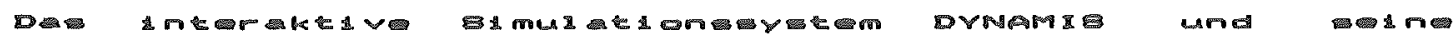

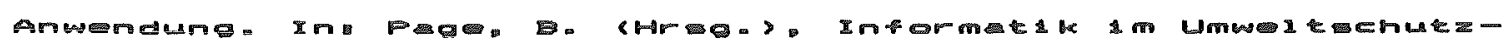

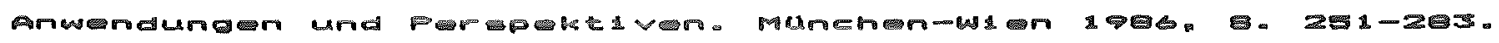

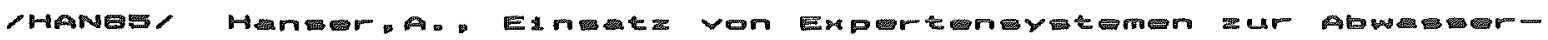

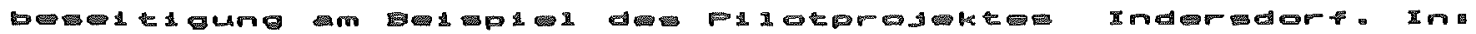

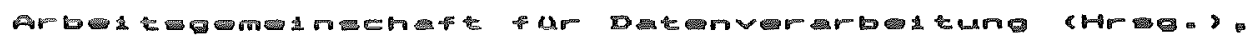

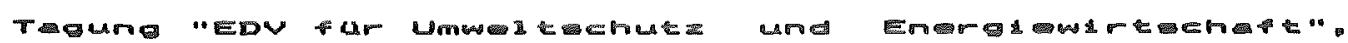

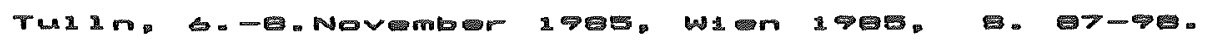

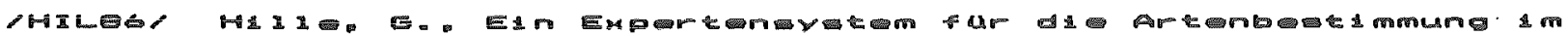

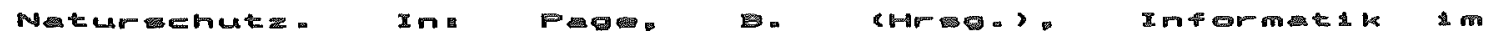

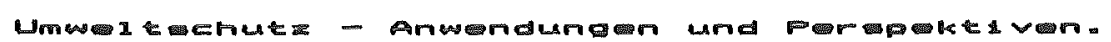

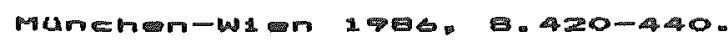

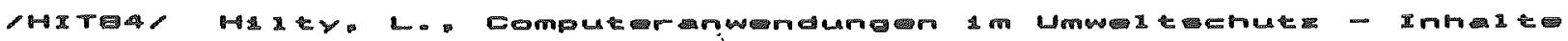

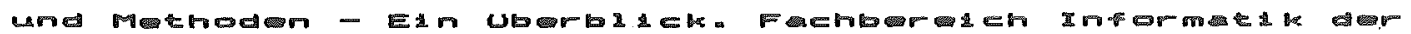

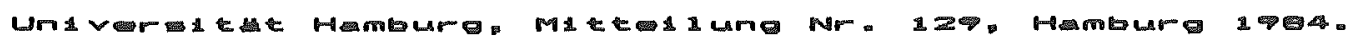

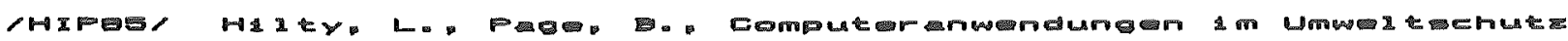

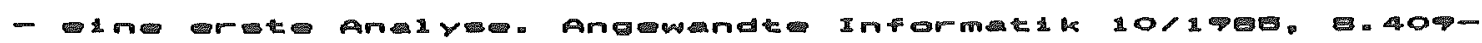
410

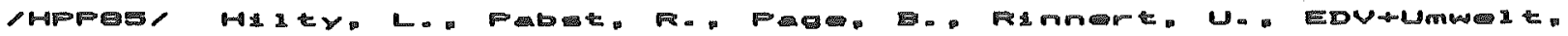

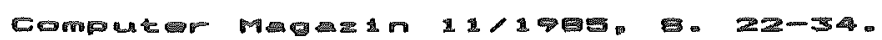




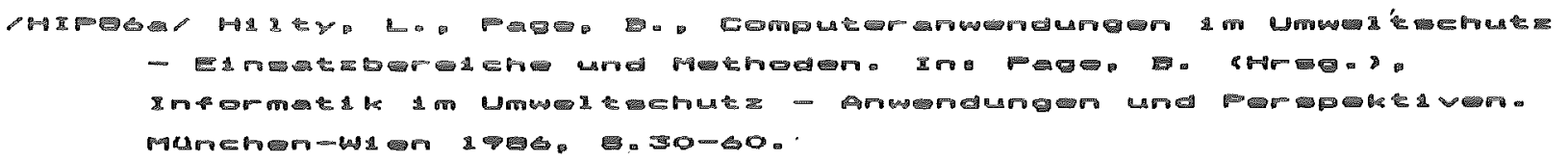

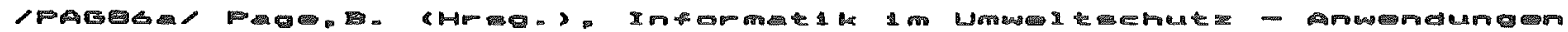

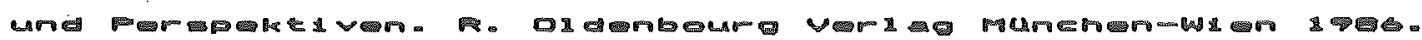

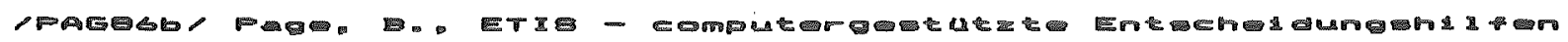

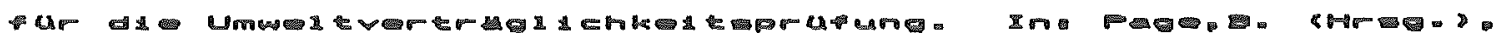

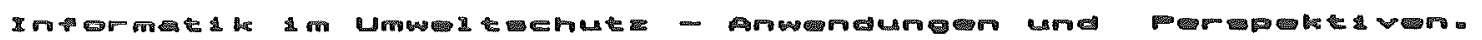

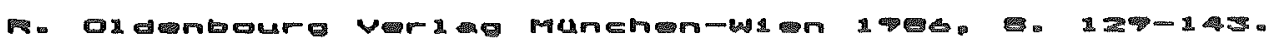




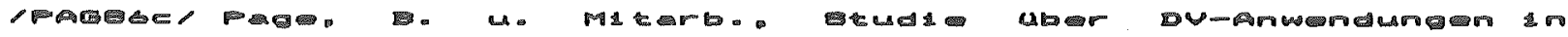

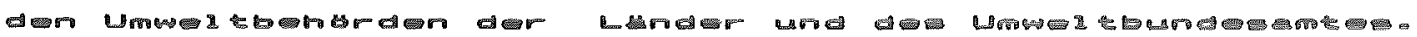

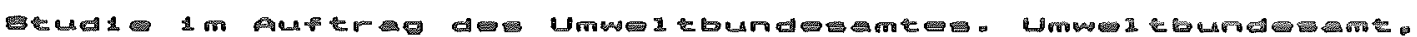

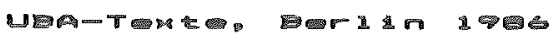

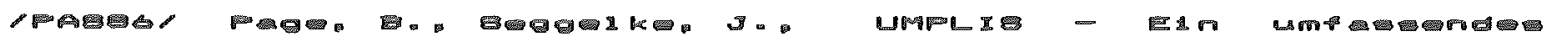
Intormet

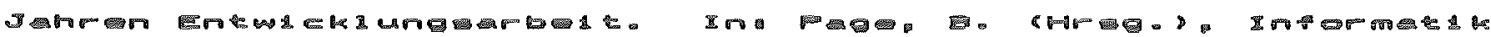

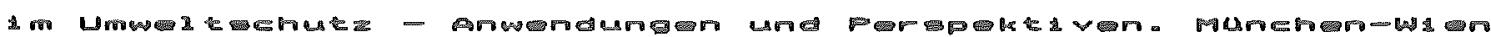

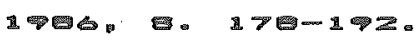

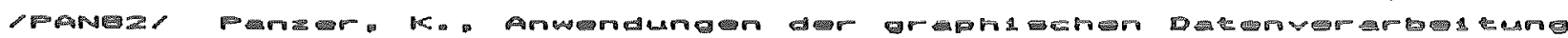

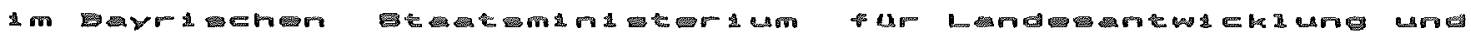

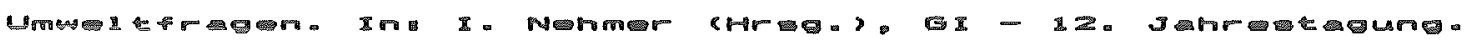

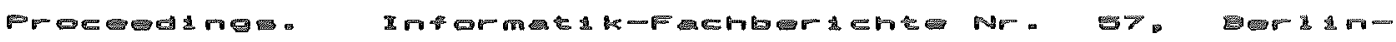

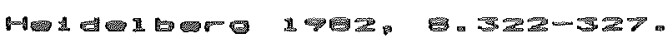

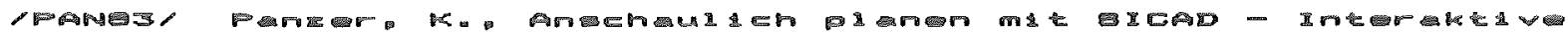
Gr:

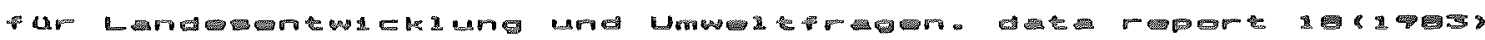

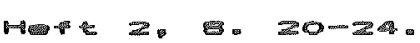

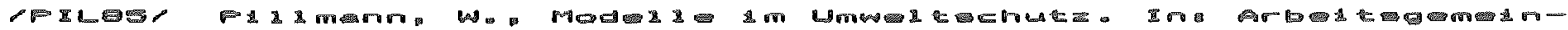

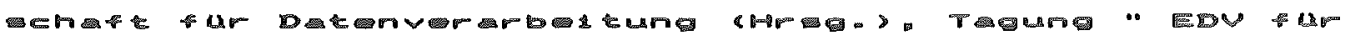

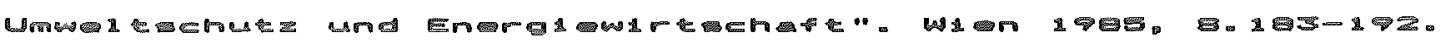

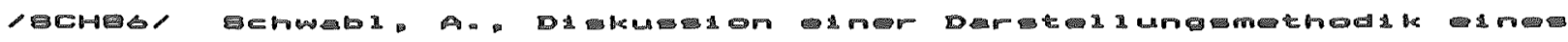

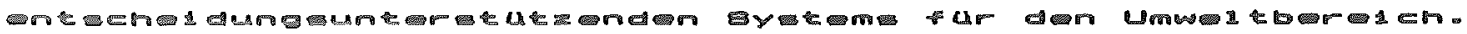

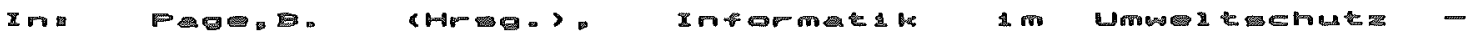

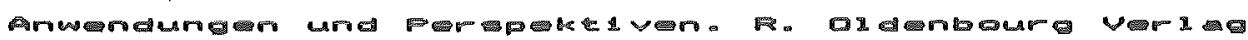

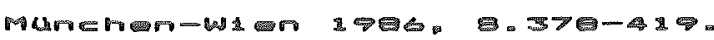

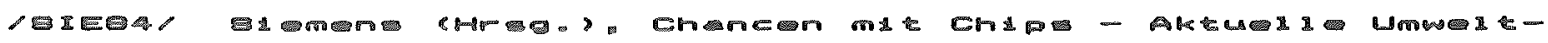

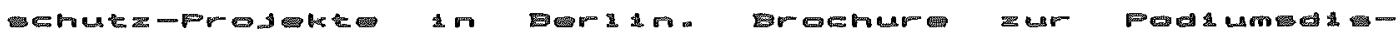

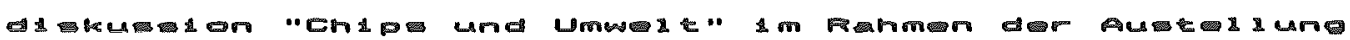

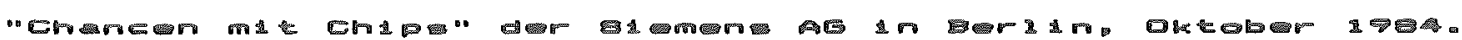

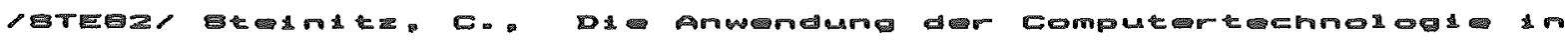

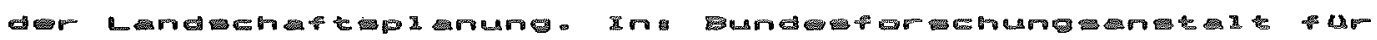

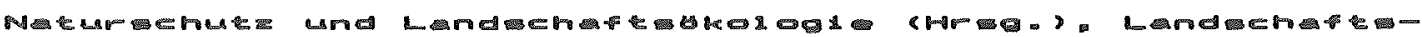

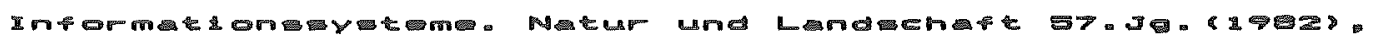
H. 12.8 .42

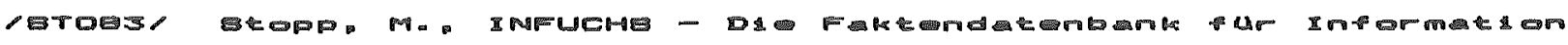

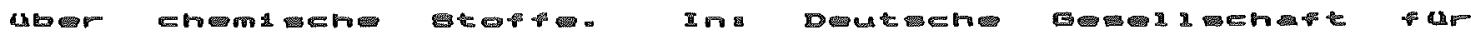

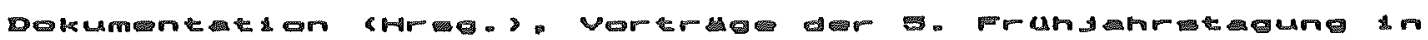

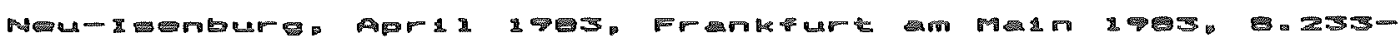
$2 \mathrm{~s}$.

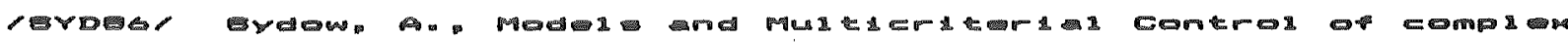

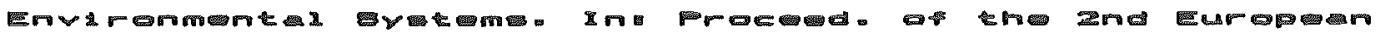

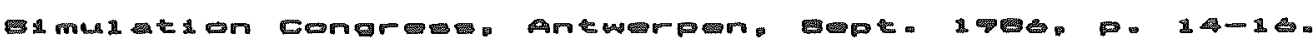




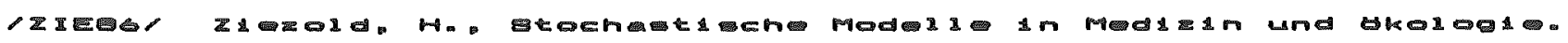

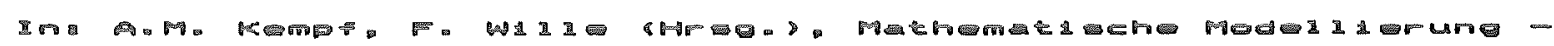

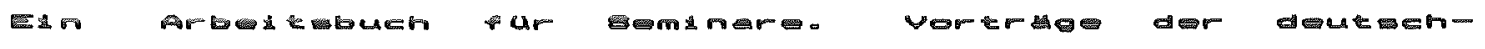
from

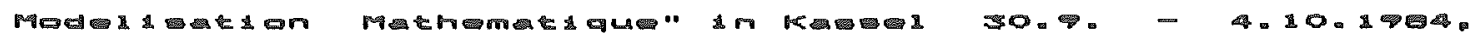

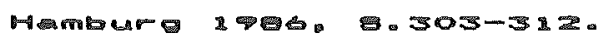


Konzeption für das Umweltinformationssystem in Baden-Württemberg (UIS)

D. Bartl

Staatsministerium Baden-Württemberg

Leiter des Bereichs Technologie in der Stabsstelle

für Information und Kommunikation

Richard-Wagner-Str. 15, 7000 Stuttgart 1

\section{Gliederung:}

1. Einleitung

1.1 Landessystemkonzept

1.2 Landesinformationssystem (LIS)

2. Konzeption

2.1 Definition und Zielsetzung

2.2 Komponenten des UIS

2.2.1 Grundkomponenten des UIS

2.2.2 Zusätzliche Komponenten des UIS

2.2.3 Ressort- und länderübergreifende Umwelt- und Fachdaten 


\section{EINLEITUNG}

Im Auftrag der Landesregierung hat die Arbeitsgemeinschaft DIEBOLD-DORNIER-IKOSS im Dezember 1984 ein Gutachten zur "Erstellung eines Landessystemkonzeptes (LSK) für einen rationellen und wirtschaftlichen Einsatz der Informations - und Kommunikationstechniken in der öffentlichen Verwaltung des Landes Baden-Württemberg" vorgelegt.

Als Einzelszenario (8) "Umweltinformationssystem (UIS)" enthält das Gutachten ein Konzept für den Einsatz der Informations - und Kommunikationstechniken zur Bearbeitung der Aufgaben des Ministeriums für Emährung, Landwirtschaft, Umwelt und Forsten (Umweltministerium-EM), die dem Umweltschutz zuzurechnen sind.

Der Ministerrat hat in seiner Sitzung am 14./15.01.1985 das Gutachten als grundsätzliche Entscheidungshilfe für den weiteren Ausbau der Informationstechnik in der Landesverwaltung begrübt und die Minister en zu konkreten Stellungnahmen aufgefordert. Zur Auswertung der Stellungnahmen und Vorbereitung der grundsätzlichen Entscheidung wurde im Staatsministerium eine Projektgruppe LSK eingesetzt. Diese Projektgruppe LSK hat einen Bericht erarbeitet, der dem Kabinett am 15. Juli 1985 vorgelegt wurde. Auf dieser Sitzung hat der Ministerrat die Vorschläge des Staatsministeriums über grundlegende Maßnahmen zum Aufbau des Landessystemkonzepts gebilligt und das EM beauftragt, die Konzeption für ein Umweltinformationssystem zu erstellen.

Das EM hat hierzu eine Projektgruppe UIS eingesetzt und deren Leitung übernommen. Die Geschäftsführung und die informationstechnische Begleitung erfolgte durch die Landesanstalt für Umweltschutz Baden-Württemberg (LfU). Zur Ermittlung des Bedarfs und der Anforderungen an ein UIS führte die Projektgruppe im Ressortbereich des EM eine Befragung über die Aufgaben mit Umweltbezug insbesondere in den Bereichen Abfall, Boden, Lärm, Lebensmittel, Luft, Natur und Landschaft, Płlanze, Radioaktivität, Tier, Umweltchemikalien, Umweltökonomie, Wald und Wasser sowie über die vorhandene und gaplante Nutzung der Informations - und Kommunikationstechniken durch. Einige ausgewählte Aufgaben des Statistischen Landesamtes (5taLa) und des Sozialministeriums (SM) konnten bereits in die Befragung mit einbezogen werden. 
Im Hinblick auf die Komplexität des Vorhabens wird zuerst eine Ausbaustufe des UIS im Sinne einer informations- und kommunikationsgestützten Aufgabenerledi. gung bei den Dienststellen im EM-Geschäftsbereich dargestellt. Erst auf dieser Grundlage kann in weiteren Ausbaustufen das Gesamtspektrum des UIS unter Einbeziehung aller Ressorts und des kommunalen Bereichs abgedeckt werden.

Die Projektgruppe UIS, in der das StaLa ständig und das SM zu einzelnen Punkten vertreten waren, hat ihr Konzept in Abstimmung mit der Stabsstelle für Information und Kommunikation beim Staatsministerium erstellt. Auf die Beteiligung der übrigen Ressorts und der kommunalen Landesverbände wurde in dieser Konzeptionsphase (UIS als ressortspezifisches Szenario entsprechend dem LSK) verzichtet. Die kommunalen Landesverbände erhalten den Bericht der Projektgruppe zur Kenntnisnahme.

\subsection{Landessysternkonzept (LSK)}

Nach dem Beschlub des Ministerrats vom 14./15.01.1985 waren folgende im LSK-Gutachten vorgeschlagene 4 Einzelprojekte sofort in Angriff zu nehmen:

- Führungorientierung des Informationswesens (FOD

Durch dieses Projekt soll erreicht werden, daß die Führungskräfte in den Ministerien des Landes besser und schneller informiert werden und die Effizienż der Arbeit insbesondere durch den Einsatz moderner Bürotechniken sowie den AnschluB an Informationssysteme wie z.B. LIS und UIS gesteigert wird.

- Haushaltrmanagementsystem (HMS)

Das HMS hat die automatisierte Abwicklung von Haushaltsplanerstellung, Haushaltsvollzug und Kassenwesen zum Ziel; zugleich bekommt die Verwaltung Instrumente in die Hand für die präzise Durchführung von Planungen mittels alternativer Modellrechnungen, für die flexible Gestaltung des Vollzugs und für die effektive Kontrolle des Vollzugs. Die Automatisierung der Mittelbewirtschaftung verspricht einen hohen Rationalisierungsgewinn. Das HMS wird auch für die Mittelbewirtschaftung bei der Erledigung von Umweltaufgaben eingesetzt werden. 
- Bïrosutomation bei den Regierungepräsidien (BAR)

Durch die Ausstattung mit Büroinformationssystemen soll bei den Regierungspräsidien (RP) die Erfüllung der Verwaltungsaufgaben rationalisiert und qualitativ verbessert werden. Die Bündelungsfunktion der RP, die auch viele Aufgabenbereiche des EM umfaßt, soll damit gestärkt werden. Die RP werden im Rahmen ihrer Fachaufgaben einen Zugriff auf das UIS erhalten.

- Bürosutomation bei den Familiengerichten (BAF)

Danach sollen die Geschäftsstellen der Familiengerichte mit Büroautomationssystemen ausgestattet werden. Ein unmittelbarer Bezug zum UIS besteht nicht.

Von den übrigen Vorschlägen des LSK-Gutachtens sollen laut MinisterratsbeschluB vom 15.07.1985 das UIS und die folgenden Einzelprojekte weiter untersucht werden:

- Netzkonzeption firi die Landesverwaltung (NKL)

Die Entwicklung einer Netzkonzeption ist von vorrangiger Bedeutung. Sie enthält einerseits die Festlegung von Standar Js und Schnittstellen, die unabdingbar sind für eine umfassende technische Kommunikation. Sie muB auch die Entwicklung bei Postnetzen und -diensten sowie bei den internationalen Normungsgremien berücksichtigen. Die NKL soll ferner die zukünftigen Wege der Information und Kommunikation in der Landesverwaltung festlegen und ist damit eine wesentliche Komponente für die Weiterentwicklung des UIS.

Inzwischen wurde eine Konzeptstudie für ein Landesverwaltungsnetz erstellt. Die Rechenzentren sollen auf SNA/SDLC-Betrieb umgestellt werden. In der nächsten Phase ist die Netz-Integration geplant. Das Umweltrechenzentrum der Landesanstalt für Umweltschutz Baden-Württemberg (DV-LfU) soll zunächst von der Umstellung ausgenommen bleiben und über ein Gateway eingebunden werden. Damit ist gewährleistet, daB der Betrieb der bestehenden Meßnetze unbeeinträchtigt bleibt. Ein Netz-ManagementZentrum soll Steuerungs - und Beratungsfunktionen landesweit wahrnehmen. 
- Regierungamenagement-System (RMS)

Ein RMS soll die Landesregierung beim Beschaffen und Auswerten bon Informationen unterstützen und die ressortübergreifende Planung erleichtern und verbessern helfen. Dazu muß es möglich sein, Informationen auch aus dem UIS führungsorientiert abzufragen und darzustellen.

\subsection{Landesinformationssystem (LIS)}

Mit Beschluß vom 15. Juni 1985 beauftragte der Landesausschuß für Information (LAl) das StaLa in Zusammenarbeit mit der Landtagsverwaltung im Rahmen des Landesinformationssystems (LIS) ein Teilkonzept für ein Umweltinformationssystem zu erarbeiten. Da die Landesregierung am 15. Juli $1985 \mathrm{im}$ Rahmen der Arbeiten zum Landessystemkonzept ebenfalls einen Auftrag für die Erstellung eines Umweltinformationssystems erteilte, galt es, beide Systeme gegeneinander abzugrenzen und zu koordinieren.

Um Doppelarbeit zu vermeiden und um die Koordinierung beider Vorhaben zu erleichtern sowie den gegenseitigen lnformationsa sstausch zu gewährleisten, wurde das Statistische Landesamt in die Projektgruppe UIS des EM aufgenommen.

Zur eindeutigen Unterscheidung werden die Projekte wie folgt benannt:

Im Bereich des Landessystemkonzepts Umweltinformationssystem (UIS) und im Bereich des Landesinformationssysterns LIS-Bereich Umwelt.

Aufgabe des LI5-Bereich Umwelt ist es, Fakten und Nachweise zu sammeln und jedermann zur Verfügung zu stellen.

Die Faktendatenbanken der Struktur - und Regionaldatenbank (SRDB) sollen um umweltrelevante Datenbestände ergänzt werden durch:

- Pflege und Ausbau der Umweltstatistiken

- Übernahme aggregierter, sachlich überprüfter und aufbereiteter Daten aus dem UIS.

Mit der Prüfung der Übernahmefähigkeit soll ein Unterausschuß aus Vertretam der Landtagsverwaltung, des StaLa und des EM beauftragt werden. 
Im Sinne einer kostensparenden Arbeitsteilung wird von der Realisierung folgender Nachwe issysteme beim LIS-Bereich Umwelt ausgegangen:

- In den Datenbanken der Landesbibliographie ist mit Unterstützung beider Landesbibliotheken das regionalbezogene umweltrelevante Schriftgut nachzuweisen; es ist bei den Bibliotheken zu archivieren. Die Vereinbarungen des Bund/Länderarbeitskreises Umweltinformationssysteme (BLAK) über die Erfassung und Speicherung von Umweltliteratur werden hierbei berücksichtigt.

- Das automatisierte Vorschriftenverzeichnis Baden-Württamberg ist um umweltrelevante Vorschriften zu erweitern, die Bundes- und EG-Recht nachweisen. Die Herausgabe einer eigenen Vorschriftensammlung ist anzustreben.

- Für den Nachweis von bundesrechtlichen Vorschriften und Gerichtsentscheidungen soll eine Übernahme von Nachwe:sen aus dem Juristischen Informationssystem der Bundesrepublik Deutschland (JURIS) angestrebt werden.

Zusätzlich werden in das UIS die bestehenden Datenbanken des LIS eingebracht:

- SRDB mit zur Zeit über 100.000 Daten je Gemeinde

- Parlamentgdokumentation des Landtags (ADAK-BW) mit dem Nachweis der parlamentarischen Aktivitäten

- Vorschriftendokumentation zum Landesrecht (AVV-BW) mit dem Nachwe is des geltenden Landesrechts

- Landesbibliographie mit dem Nachweis von Literatur, die sich mit Baden-Württemberg befaßt (hierbei wird auch die Literatur mit Umweltbezug berücksichtigt)

Durch den Zugriff und die Mitbenutzung dieser Datenbanken kann das UIS hier auf eine eigene Datensammlung verzichten. 


\section{Konzepicion}

\subsection{Definition und Zielsetzung}

Das Umweltinformationssystem ist ein aufgabenorientiertes, organisatorisches und informationstechnisches Rahmenkonzept für die Bereitstellung von Umweltdaten und die Bearbeitung von fachbezogenen und fachübargreifenden Aufgaben im Umweltbereich. Die grundsätzliche Konzeption des UIS zeigt Bild 1, Seite 10.

Zielsetzung des UIS ist zunächst, die Aufgabenerfüllung innerhalb der Bereiche des EM und der nachgeordneten Dienststellen mit Umweltbezug durch einen rationellen und wirtschaftlichen Einsatz der Informations - und Kommunikationstechniken $2 u$ verbessern.

Durch die Ermittlung, Speicherung und unmittelbare Bereitstellung von punktuellen und landesweiten Umweltdaten, die für die Qualität der Umweltgüter und insbesondere für die Schadstoff- und Belastungssituation für Baden-Württemberg von Bedeutung sind, soll es die ständige aktuelle Verfolgung der Umweltsituation ermöglichen.

Für die fachübergreifende und interdisziplinäre Aufbereitung und Bewertung von Umweltdaten sind Hilfsmittel wie mathematische, statistische und graphische Verfahren und Modelle unter dem Einsatz von Datenbanken vorzusehen, in denen Umweltdaten in einem einheitlichen Bezugssystem gespeichert sind. Insgesamt kann mit Hilfe des UIS die Umweltvorsorge im Sinne eines aktiven Umweltschutzes wesentlich verbessert werden.

Das UIS dient damit auch der Unterstützung der politischen Planungs- und Entscheidungsprozesse im Umweltbereich. Darüber hinaus leistet es durch stark aggregierte und kommentierte Daten und Graphiken einen wichtigen Beitrag zur Information der Öffentlichkeit. 


\section{UMWELTINFORMATIONSSYSTEM BADEN-WÜRTTEMBERG \\ UIS}

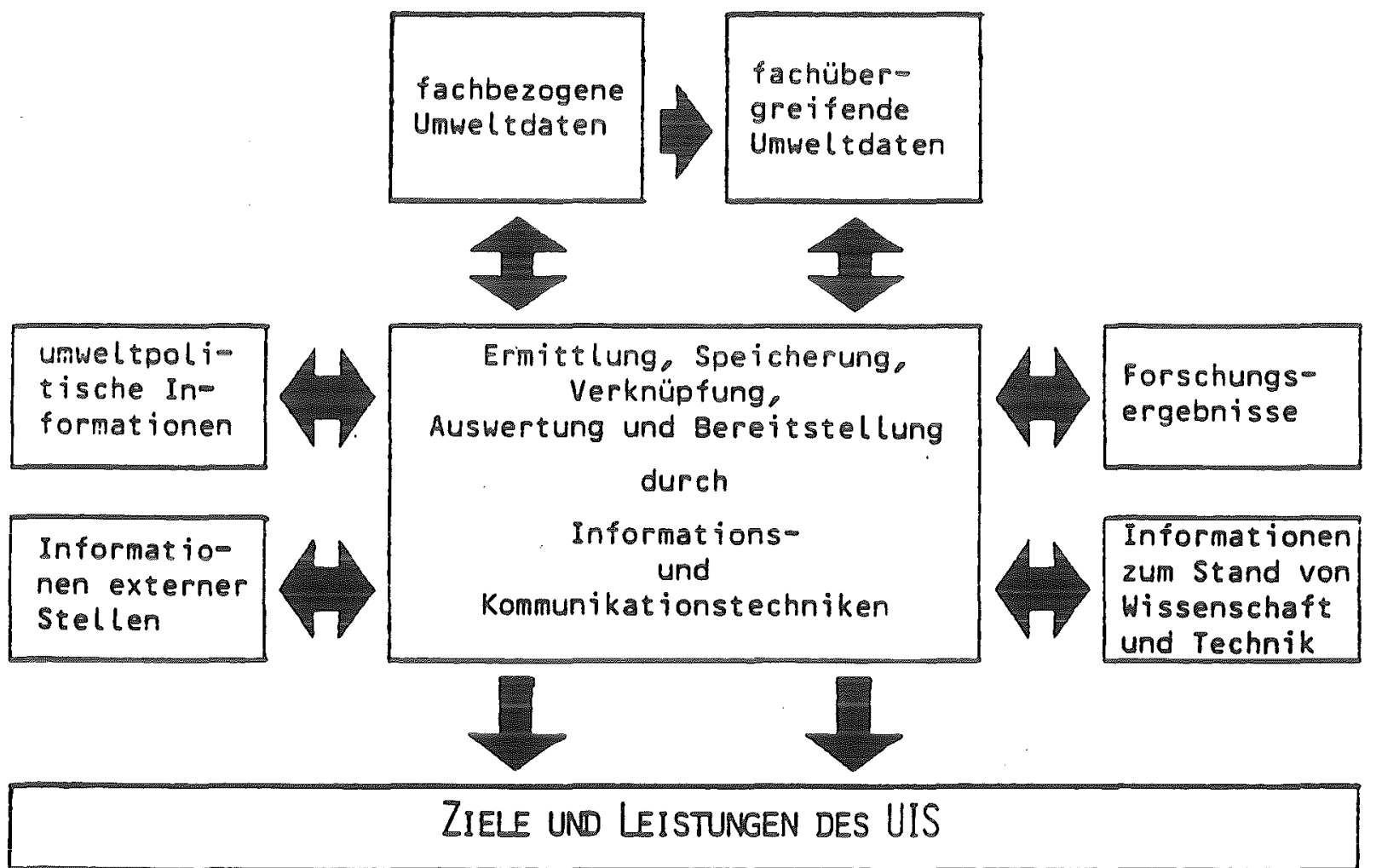

- Effektive Erledigung von Unweltaufgaben bei fationellem Einsatz der Informations- und Kommunikationstechniken

- Unterstützung der politischen Planungsm und Entscheidungsprozesse in Umwelebereich

- Landesweite oder punktuelle Auswertungen von Belastungszuständen

- Uberwachung der Anwendung unweltrelevanter Gesetze, Verordnungen und sonstiger Umweltvorschriften

- Erfolgskontrolle der getroffenen Maßnahmen

- Aufbau von benutzerfreundtichen Datenbanken

- U. 


\section{$2.2 \quad$ Komponenten des UIS}

\subsubsection{Grundkomponenten des UIS}

Das UIS baut auf den fachbezogenen und fachübergreifenden Umweltaufgaben der Dienststellen des EM auf. Die hierfür einzusetzenden informations - und kommunikationstechnischen Verfahren sind die Grundkomponenten des UIS.

In allen Umweltbereichen werden landesweite und punktuelle Umweltdaten erhoben. Die Erfassung, Strukturierung und Speicherung dieser Daten in Arbeitsdateien richtet sich zunächst nach den fachlich vorgegebenen Arbeitsschwerpunkten der Dienststellen. Für fachübergreifende Auswertungen stellen diese Daten die wesentliche Grundlage dar. Ein erheblicher Teil dieser Umweltdaten wird bereits mit Hilfe von automatisierten Verfahren unter Einsatz der Informations - und Kommunikationstechniken bearbeitet. Diese Umwaltdaten sind in digitaler Form in Arbeitsdateien gespeichert und stehen für das UIS zur Verfügung.

Von hervarragender Bedeutung für das UIS sind die in den Umweltbereichen Luft, Wasser, Boden und Radioaktivität betriebenen oder geplanten automatischen Meßnetze. Sie ermöglichen eine laufende und aktuelle Verfolgung der bereichs. spezifischen Schadstoffsituation. Die Speicherung der Meßnetzdaten in langjährigen MeOreihen ist zugleich Ausgangspunkt und Datenbasis für eine umfassende Beurteilung von Veränderungstendenzen der Umweltsituation.

Für Aufgaben, bei denen Datenbestände und Umweltinformationen in herkömmlicher Weise in Akten, Karteien oder kartenmäßig festgehalten werden, sieht das UIS den beschleunigten Einsatz der Informations- und Kommunikationstechniken vor. 


\subsubsection{Zusätzliche Komponenton des UIS}

Neben den Grundkomponenten sieht die Konzeption des UIS eine Reihe von übergreifenden Komponenten vor, die der Zusammenfassung, Bereitstellung und Ubermittlung von relevanten Umwelt daten und -informationen dienen. Durch sie werden die Grundkomponenten darüberhinaus in einen gemeinsamen Rahmen eingebettet, der auf Abstimmung und Informationstransparenz ausgerichtet ist. Damit soll auch ein rationeller und wirtschaftlicher Einsatz der Informationsund Kommunikationstechniken im Geschäftsbereich des EM erreicht werden. Die zusätzlichen Komponenten stellen somit eine wesentliche Voraussetzung für das Rahmenkonzept des UIS dar.

Zusätzliche Komponenten des UIS sind Datenbanken mit aggregierten Umweltdaten sowie mit umweltpolitischen, technisch-wissenschaftlichen und rassortund landesübergreifenden Umweltinformationen, die für die Fachbehörden und für die umfassende Unterstützung der politischen Planungs - und Entscheidungsebene von Bedeutung sind. Bei der Entwicklung des UIS werden soweit wie möglich bereits vorhandene Umwelt - und Fachdatenbanken anderer Einrichtungen einbezogen. Durch die Speicherung von stark aggregierten und kommentierten Daten und Graphiken können die zusätzlichen Komponenten auch einen wichtigen Beitrag für die Information der Óffentlichkeit leisten.

Entscheidend für die Funktionsfähigkeit des UIS ist, neben der Einrichtung von Datenbanken und Umweltdateien bzw. dem Zugriff auf sie, der Informationsverbund zwischen den Dienststellen im vorgesehenen Landesverwaltungsnetz.

Für das UIS sind folgende zusätzlichen Komponenten von Bedeutung:

\begin{tabular}{|c|c|c|}
\hline & Umweltdatenkatalog & $(D V-E M)$ \\
\hline - & Methodenkatalog & $(D V-E M)$ \\
\hline - & $\begin{array}{l}\text { Katalog der DV-Installationen } \\
\text { und DV-Anwendungen des EM }\end{array}$ & $(B K S-E M)$ \\
\hline - & Umweltpolitische Informationen & $(B K S-E M)$ \\
\hline & Umwelt datenbanken & $(D V=L f \cup, D V=E M)$ \\
\hline - & Daten zur Umweltqualität & $(D V=L f U)$ \\
\hline - & Methodenbank & $(D V-L f U)$ \\
\hline - & Forschungsergebnisse & $(D V-L f U)$ \\
\hline - & $\begin{array}{l}\text { Daten zum Stand der Wissenschaft und } \\
\text { zu Stand und Regel der Tachnik }\end{array}$ & $(D V-L P U)$ \\
\hline
\end{tabular}




\section{Umweltdatenkatalog:}

Der Umweltdatenkatalog ist ein umfassendes Übersichtsverzeichnis über die Gesamtheit der bei den Fachbehörden erhobenen und gespeicherten Umweltdaten. Durch ihn soll die Transparenz der vorhandenen Umweltdaten erhöht und ihre Verwendbarkeit für fachübergreifende Aufgaben erleichtert werden. Damit lassen sich informationslücken besser erkennen und mit den Komponenten des UIS leichter schlieBen. Das mehrfache Erfassen und Speichern von Umweltdaten wird vermieden und aufwendige MeBaktionen werden auf das notwendige Maß beschränkt.

Der Umweltdatenkatalog stellt gleichzeitig eine Informationsgrundlage für den BLAK dar.

\section{Methodenkatalog:}

Der Methodenkatalog stellt ein Verzeichnis dar, in dem die fachbezogenen und fachübergreifenden DV-Methoden (DV-Hilfsprogramme und speziffische DV-Verfahren) der Dienststellen nach einem einheitlichen Schema dokumentiert sind. Die lauffähigen Programmroutinen liegen auf den dezentralen bzw. zentralen DV-Systemen. Durch den Methodenkatalog soll die Übersicht über die vorhandenen mathematischen, statistischen und graphischen Verfahren und Modelle erhöht und ihre gemeinsame Nutzung für Umweltaufgaben erleichtert werden. Die redundante Programmentwicklung gemeinsam nutzbarer Methoden wird vermieden.

Auch der Methodenkatalog ist eine wichtige Informationsgrundlage für den BLAK.

\section{Katalog der DV-lnatallationen und DV-Anwendungen:}

Im Bürokommunikationssystem des EM (BKS-EM) wird ein Katalog aller DV-Installationen und DV-Anwendungen geführt, der Teil der ressortinternen DV-Gesamtplanung ist. Gleichzeitig bietet er anderen Ressorts und Dienststellen die Möglichkeit, Ressourcen zu erfragen und gegebenenfalls mitzubenutzen. 


\section{Ummelkpolitiache Intormationen:}

Es werden umweltpolitische Daten aufbereitet und gespeichert, die als Führungsinformationen für das Land Baden-Württemberg von Bedeutung sind. Sie umfassen U.a. die Umweltpolitischen Programme (MUP).

\section{Umweltdatenbanken:}

Die bei den fachbezogenen Umweltaufgaben erhobenen Umweltdaten stellen die Datengrundlage für die fachübergreifende und interdisziplinäre Bewertung komplexer Umweltprobleme dar. Hierzu werden die Daten der verschiedenen Umweltbereiche nach übergeordneten Gesichtspunkten aggregiert, einheitlich strukturiert und in Umweltdatenbanken zusammengeführt. Es werden jedoch nicht sämtliche erhobenen Umweltdaten auch zentral gespeichert. Die Ubernahme von Umweltdaten richtet sich nach den konkreten Erfordernissen.

Punkt-, linien- und flächenbezogene Umweltdaten nüssen in einem Bezugssystem gespeichert und fortgeschrieben werden.

Von großer Bedeutung für die gezielte Nutzung der Umweltdatenbanken ist die frühzeitige Abstimmung der Datenstsöme. In vielen Fällen werden sich durch notwendige Normierungen Rückwirkungen auf die Meß - und Erfassungsarbeit der einzelnen Fachbereiche ergeben.

\section{Daten zur Unweltqualität:}

Hier erfolgt die Speicherung von verdichteten Umweltdaten und -ergebnissen mit Graphiken und erklärenden Kommentaren, die von den Dienststellen gewonnen werden. Damit werden die Voraussetzungen für eine DV-unterstützte Umweltberichterstattung geschaffen. 


\section{Methodorbank:}

Die für die fachübergreifende Bewertung der Umweltsituation notwendigen mathernatischen, statistischen und graphischen Hilfsmittel werden in einer zentralen Methodenbank zusammengefaßt.

Diese zentrale Methodenbank ist zusammen mit den Umweltdatenbanken U.a. Grundlage für die Entwicklung von Expertensystemen im Umweltbereich.

\section{Forschungsergebnisso:}

Die Umweltforschung wird vom Land Baden-Württemberg mit jährlich 27 Mio. DM gefördert. Diese Forschung wird von der Zentralen Koordinierungsstelle für Umweltforschung (ZKU) in der LfU koordiniert. Wichtige Ergebnisse und Zwischenergebnisse der Umweltforschung werden als Grundlage für die Expertensysteme aufgenommen.

Daten zum Stand der Wissenschaft und zu Stand und Regel der Technik:

Die möglichst objektive Erfassung und Bewertung der Umweltqualität erfordert, verfügbare Informationen über den Stand von Wissenschaft und den Stand und Regel der Technik abrufbereit zu halten und ständig zu aktualisieren. Nur so können die Dienststellen ihrer Aufgabe zur maximal möglichen Minderung und Vermeidung von Umweltbelastungen nachkommen.

Die Informationen sind wichtige Hilfsmittel für die Vorbereitung sachgerechter politischer Entscheidungen, die Erteilung von dem Stand bzw. Regel der Technik entsprechenden Auflagen bei der Genehmigung umweltrelevanter Anlagen sowie für die Vergabe von Forschungs- und Entwicklungsvorhaben usw. 
2.2.3 Ressort - und länderibergreifende Umwell - und Fachdaten

Ein wirkungsvolles Umweltinformationssystem muß den Zugriff auf wichtige ressort - und länderübergreifende Umwelt - und Fachdaten umfassen. Im Rahmen des UIS ist daher der Zugriff auf folgende externe Datenbestände zu realisieren:

- Daten des LIS

- Lebensmittel

- Umweltforschungs - und

Literaturdaten

- Technische Daten

- Länderübergreifende Umweltdaten

- Medizinische Daten

- Juristische Daten

- Trinkwasserdaten

- weitmaschiges landesweites digitales Geländemodell

- Automatisiertes Liegenschaftskataster

- Bodenkartierungen

- Daten des Verwaltungsvollzugs
(StaLa)

(SM, CLUA; soweit nicht EM, STUA)

(UBA)

$(F \backslash Z)$

(BLAK)

(DIMDI)

(JURIS)

(SM, CLUA)

(LV)

(LV)

(GLA)

(z.B.RP)

Weitere Umwelt - und Fachdaten werden noch zu erheben sein. 
Das Umweltplanungs- und Infomationssystem des Bundes UMPLIS;

Aufgaben, Hoffnungen und Grenzen.

\author{
J. Seggelke \\ Umweltbundesamt \\ Bismarckplatz 1, 1000 Berlin 33
}

\title{
Zusammenfassung
}

Im Errichtungsgesetz des Umweltbundesamtes von 1974 sind die Aufgaben einer zentralen Dokumentation und des Informationssystems zur Umweltplanung genannt. Beides wurde in dem System UMPLIS zusammengefaßt, das U.a. aus der wissenschaftlichen Dokumentation $(F+E-V o r-$ haben und Fachliteratur), Faktendatenbanken (vor allem Umweltchemikalien, Umweltbeobachtung / Monitoring und Stand der Umwelttechnik) einer grafischen Umweltdatenbasis und einer Methodenbank Umwelt besteht. Wichtige im Aufbau befindliche Systeme sind dabei eine Unfallschnellauskunft (Gefahrgüter) und ein Smogfrühwarnsystem. Als Datenbankverwaltungssytem wird vor allem ADABAS verwendet; als große Anwendersysteme werden SLIM, INFUCHS und DOKSIS entwickelt. Als Grafiksystem wird SICAD eingesetzt. Neben der Beschreibung der wichtigsten Datenbanken werden das Hardware- und Softwarekonzept sowie die weitere Zielsetzung behandelt. Dabei spielen einfache Mensch-Maschine-Dialoge, die um grafische Komponenten erweitert werden, eine zentrale Rolle für die Akzeptanz vor allem bei gelegentlichen Benutzern.

\section{Gliederung}

1. Allgemeines zu UMPLIS und Stand der Nutzung

2. Wissenschaftliche Dokumentation (F+E-Vorhaben UFORDAT und Fachliteratur ULIDAT)

3. Faktendatenbanken bei UMPLIS

3.1 INFUCHS, das System für chemische Stoffe

3.2 System zur Luftqualität LIMBA

3.3 Emissionsursachenkataster EMUKAT

3.4 Abfallwirtschaftsdatenbank AWIDAT

3.5 Gewässerkundliche Datenbank HYDABA

3.6 Bibliotheksdatenbank BIBLIODAT

3.7 Grafische Umweltdatenbasis GRAFU

4. Neue Aktivitäten hoher Priorität

4.1 Smogfrühwarnsystem für die Bundesrepublik Deutschland

4.2 Waldschadensinformationssytem

4.3 Informationssystem für Schäden an Baudenkmälern und Kulturgütern

4.4 EDV-Einsatz für die Forschungsplanung im Umweltbundesamt

4.5 Schnellauskunft für gefährliche Stoffe und gefährliche Güter

4.6 Integriertes EDV-System für Fachliteratur, Forschungsvorhaben und Bibliothek

5. Hardware des Umweltbundesamtes

6. Überlegungen zu einem Softwarekonzept von UMPLIS

7. Literaturverzeichnis 


\section{Allgemeines zu UMPLIS und Stand der Nutzung}

Im Umweltbundesamt wird das Umweltplanungs- und Informationssystem (UMPLIS) aufgebaut und geführt. Seit 1974 werden von UMPLIS umweltbezogene Informationen gesammelt und gespeichert, um sie den Behörden von Bund, Ländern und Gemeinden, der Wissenschaft und der Wirtschaft sowie der interessierten Öffentlichkeit zugänglich zu machen. UMPLIS soll insbesondere die Aufgaben der politischen Planung unterstützen und ist informationstechnisches Instrument des Umweltbundesamtes, das die Bundesregierung in Fragen des Umweltschutzes vor allem auf den Gebieten Luftreinhalłung, Lärmbekämpfung, Wasserwirtschaft, Abfallwirtschaft, Umweltchemikalien und Ökologie wissenschaftlich berät $/ 1 /, 121,13 /$.

Nach einer Phase der großen, wenn nicht übergroßen, Erwartungshaltung für politische Informationssysteme Anfang der siebziger Jahre setzte sich dann erhebliche Ernüchterung und z.T. herbe Kritik an derartigen Systemen durch. Nunmehr kann von einer realistischen Einschätzung der EDV und Informationstechnik ausgegangen werden, wobei die positiven und auch optimistischen Aspekte gerade in der letzten Zeit eher überwiegen. Dieser Trend wird durch preiswerte Hardware (z.B. Personal Computer/PC) und den beginnenden Entwicklungsschub der Expertensysteme gekennzeichnet; Insgesamt sind jedoch erhebliche Fortschritte erforderlich, bis eine breite Nutzung in der wissenschaftlichen und politischen Beratung auf dem Umweltsektor erzielt werden kann.

Als Maß für die Nutzung wird häufig die Anzahl der Recherchen verwendet (Abb. 1). Leider gibt es in der Bundesrepublik keine eindeutige Festlegung oder Definition einer Recherche, sodaß der Vergleichbarkeit mit anderen Systemen Grenzen gesetzt sind.

Nach erheblichen Anfangsproblemen hat die Nutzung ab 1984 stark zugenommen. 1985 sind insgesamt über 14.000 Recherchen in den UMPLIS-Datenbanken durchgeführt worden. Dies entspricht einer Steigerungsrate von 23\% gegenüber 1984 und von 299\% gegenüber 1983 (siehe dazu Abb.1). Diese Steigerung wird sich vermutlich 1986 fortsetzen, wobei die Nutzung im Umweltbundesamt sicher durch bessere Benutzeroberflächen (Einfachdialog) wesentlich gesteigert werden kann.

Diese erheblichen Zuwachsraten waren 1984 insbesondere auf die Zunahme der Nutzung des grafischen Umweltsystems (GRAFU) der Methodenbank Umwelt zurückzuführen. Im Jahr 1985 beruhte der Zuwachs vor allem auf dem kontinuierlichen Anstieg der Nutzung der Umweltliteraturdatenbank im Rahmen des UMPLIS-Selbstwähldienstes. 


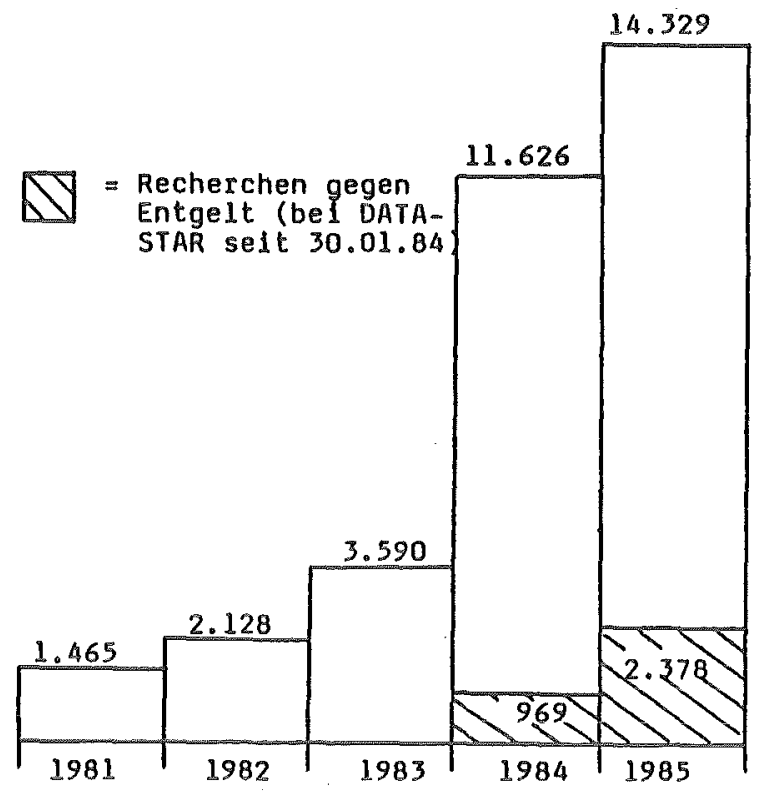

Abbildung 1: Anfragen an die UMPLIS-Datenbanken (insgesamt)

Aufbau und Betrieb von UMPLIS werden seit 1972 durch den Bund/Länder-Arbeitskreis Umweltinformationssysteme (BLAK) begleitet, in dem die Vertreter der Umweltministerien der Länder unter Vorsitz des Bundesministers für Umwelt Naturschutz und Reaktorsicherheit (BMU) vertreten sind. Die Koordinierung des Aufbaus und Betriebs von UMPLIS mit ähnlichen Systemen der Länder hat dabei eine erhebliche Bedeutung, da die Länder überwiegend die Aufgaben des Vollzuges von Umweltschutz und Umweltplanung wahrnehmen.

Seit Anfang 1984 stehen die Umweltliteraturdatenbank (ULIDAT) und die Umweltforschungsdatenbank (UFORDAT) des Umweltbundesamtes im Rahmen des UMPLIS-Selbstwähldienstes auch für den on-line-Zugriff gegen Entgelt zur Verfügung.

Beide Datenbanken werden über den Host DATA-STAR (Radio Schweiz AG, Bern) angeboten. DATA-STAR hat Ende 1985 sein Angebot an Umweltdatenbanken um die amerikanische Literaturdatenbank POLLUTION erweitert.

Ab 1987 soll auch das Informationszentrum Karlsruhe (INKA) als deutscher Knoten des International Scientific Technical Network (STN) beide Datenbanken einem weiteren Benutzerkreis im erweiterten Selbstwähldienst für den on-line-Zugriff zur Verfügung stellen. 
Die Datenbanken des Umweltbundesamtes haben sich bei DATA-STAR im Rahmen des on-lineMarktes gut eingeführt. 1985 sind 2.378 Recherchen gegen Entgelt duchgeführt worden. Die on. line-Nutzung hat somit gegenüber dem Vorjahr (seit 31.1.1984) um etwa $145 \%$ zugenommen (siehe dazu Abb.2). Ein weiterer Anstieg zeichnet sich ab.

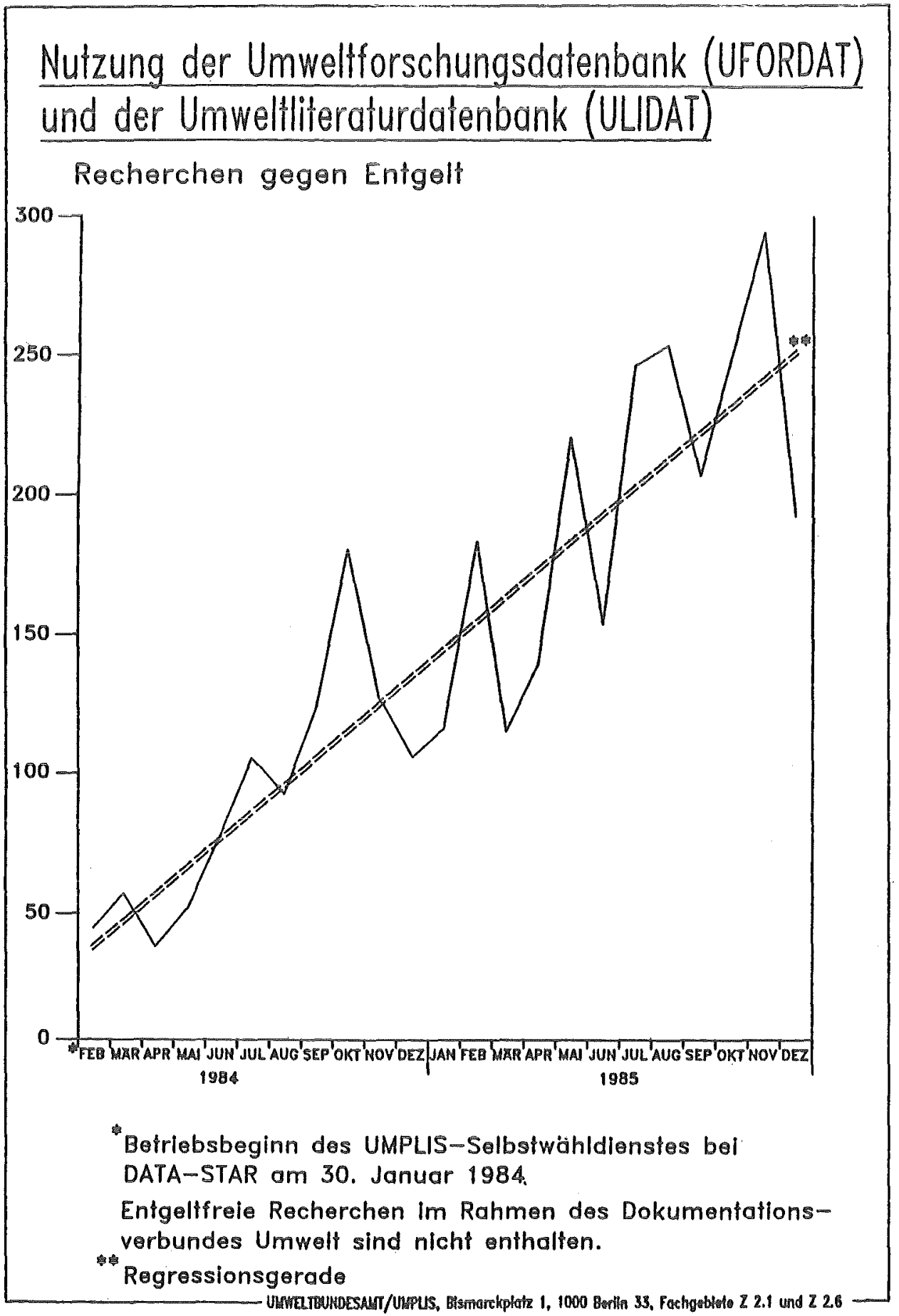

Abblldung 2 
In den ersten Monaten bei DATA-STAR haben 232 verschiedene Benutzer, davon $44 \%$ aus dem Ausland, auf die beiden Datenbanken zugegriffen. Die inländischen Benutzer setzen sich wie folgt zusammen:

- Wirtschaft $67 \%$

- Wissenschaft $22 \%$

- Verwaltung $5 \%$

- Sonstige $6 \%$

Zur Schulung der Benutzer werden regelmäßig Datenbankseminare durchgeführt. 1985 haben 11 Veranstaltungen in Berlin, Hamburg, Köln, Frankfurt, Heidelberg und München mit insgesamt 140 Teilnehmern stattgefunden. Die zweitägigen Seminare wurden von der Bertelsmann Datenbankdienste $\mathrm{GmbH}$ in München, der DATA-STAR-Vertriebsorganisation für die Bundesrepublik Deutschland und die Republik Österreich, in Zusammenarbeit mit dem Fortbildungszentrum Gesundheit und Umweltschutz e.V., Berlin, durchgeführt.

Die Bundesregierung hat 1985 die Neuorientierung ihrer Fachinformationspolitik beschlossen und mit dem Fachinformationsprogramm 1985-1988 ihre zukünftigen Ziele formuliert sowie neue Leitlinien für das Verhältnis Staat - Wirtschaft festgelegt /4/. Im Rahmen der sog. ressortspezifischen Fachinformation wird der Informationsbereich Umwelt in wesentlichen Aspekten dargestellt. Als Schwerpunktmaßnahmen werden dabei u.a. genannt:

- Verstärkung der Bund/Länder-Zusammenarbeit

- Ausweitung des on-line-Angebotes auf weitere Fachinformations-Rechenzentren

- Maßnahmen zur Steigerung der Benutzerfreundlichkeit der Datenbanken ULIDAT und UFORDAT (Erweiterung der automatischen Indexierung, Entwicklung eines mehrsprachigen Thesaurus und eines Einfachdialoges für ungeübte Benutzer)

- Ausbau der Faktendatenbanken

- Weiterentwicklung von DV-Instrumenten für die grafische Darstellung, Modell- und Prognoserechnungen sowie eines Einfach-Dialoges auch für Faktendatenbanken.

\section{Wissenschaftliche Dokumentation (F+E-Vorhaben UFORDAT und Fachliteratur ULIDAT)}

Die Datenbank für Umweltforschung UFORDAT hat jetzt einen Datenbestand von ca. 20.500 laufenden und abgeschlossenen umweltrelevanten Forschungs- und Entwicklungsprojekten und verzeichnet über 5.000 Institutionen im Umweltbereich. 
Um den treffsicheren Zugriff auf die Datenbank zu gewährleisten, wird die inhaltliche ErschlieBung der Datenlieferungen mit Unterstützung des Textanalysesystems ASTTAL vorgenommen (teilautomatische Deskribierung und maschinell unterstützte Klassifizierung). ASTTAL soll dahingehend erweitert werden, daß auch die Deskribierung englischsprachiger Texte möglich wird.

Um UFORDAT auf dem Laufenden zu halten, wurden im Rahmen des Magnetbandaustausches mit den Datenbanken DAVOR/DAKOR des Bundesministers für Forschung und Technologie sowie mit Datenbanken des Bundesministers für Ernährung und Landwirtschaft und Forsten und der deutschen Forschungsgemeinschaft Basisdaten von mehr als 1.000 Forschungs- und Entwicklungsvorhaben übernommen.

In Kooperation mit dem Österreichischen Bundesinstitut für Gesundheitswesen sind auch Vorhaben aus der Republik Österreich in die UFORDAT aufgenommen worden.

Außerdem wird eine Kooperation mit dem Bundesamt für Umweltschutz in Bern angestrebt; die ersten Schweitzer F + E-Vorhaben sind bereits zu Testzwecken gespeichert worden. Die Umweltforschungsdatenbank wird im Rahmen des UMPLIS-Selbstwähldienstes unter der DATA-STARKennung UFOR angeboten.

1985 wurden in der Umwelt forschungsdatenbank 1056 Recherchen, darunter 504 gegen Entgelt, durchgeführt (siehe dazu auch Abb.3).

Die turnusmäßige Erhebung für die 6. Ausgabe des Umweltforschungskataloges (UFOKAT 1985/86) begann Ende 1985.

Dabei werden rund 3.500 umweltrelevante Forschungsprojekte bei ca. 1.500 Forschungsnehmern überprüft und neue Forschungsvorhaben aufgenommen. Der Umweltforschungskatalog wird im Dezember 1986 fertiggestellt sein.

Die Umweltforschungsdatenbank ENREP der Europäischen Gemeinschaften (EG), zu der die Partnerstaaten jeweils ihre nationalen Daten zuliefern, wurde durch den Auftragnehmer der EG aktualisiert und verbessert. 1987 sollen dann die Daten allen Partnerstaaten in einem benutzerfreundlichen Layout über den Host ECHO der EG zugänglich gemacht werden. Weiterhin sollen die F+E-Vorhaben von Spanien und Portugal in ENREP übernommen werden. Der Zugriff soll möglichst in allen EG-Sprachen ermöglicht werden.

Eine verläßliche Dokumentation aller relevanten Vorhaben und Institutionen als Grundlage der Koordination der Umweltforschung hat eine große Bedeutung, ist aber schwierig in der Realisierung. Die Abgabe der Daten an UMPLIS kann nämlich meist nur erbeten, nicht aber erzwungen 


\section{Nutzung der Umwelfforschungsdatenbank (UFORDAT)}

Recherchen gegen Entgelt

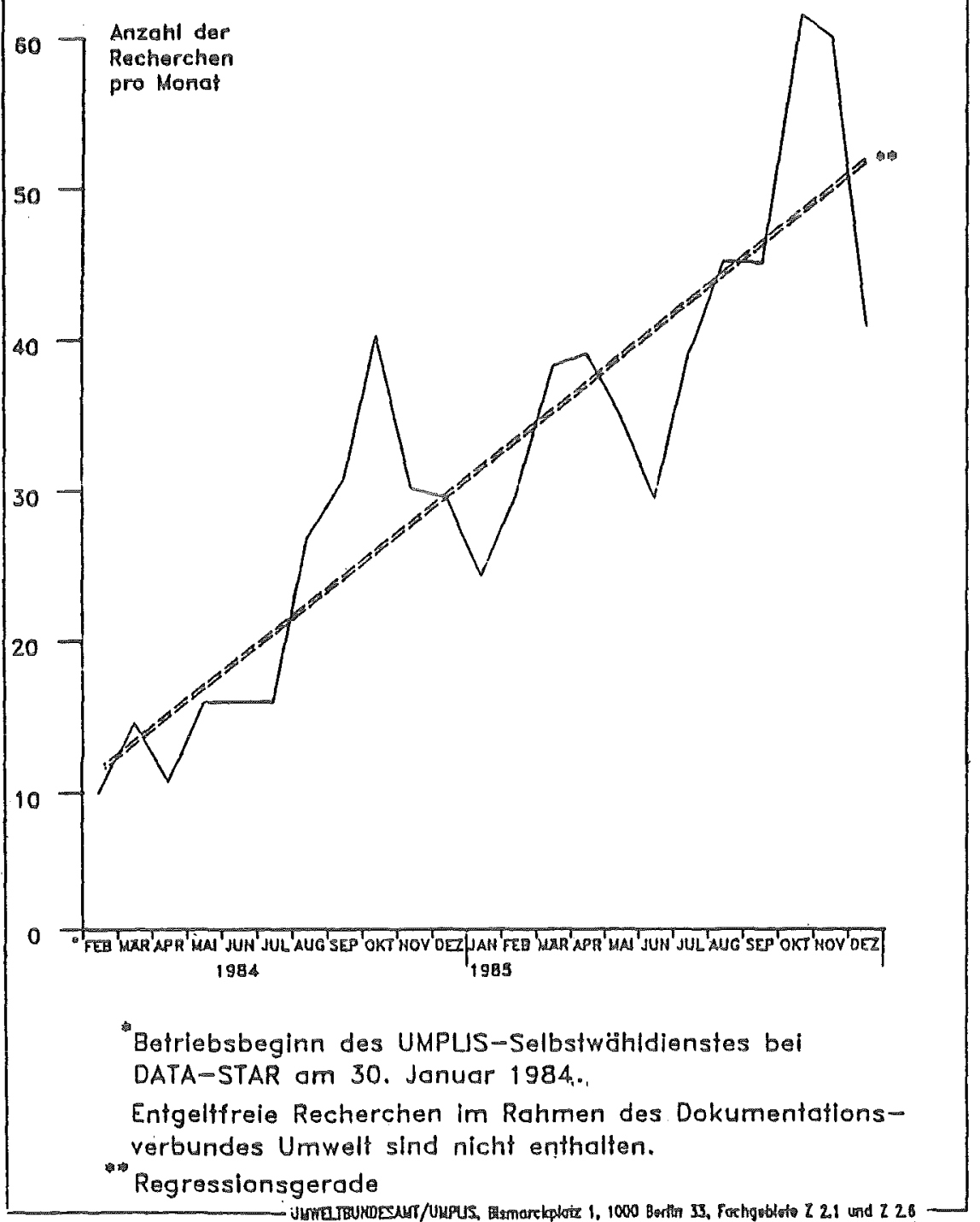

Abbildung 3

werden. Dies gilt für Vorhaben, die öffentlich gefördert werden, in erheblich stärkerem Maße jedoch für die Industrieforschung.

Die beim Host DATA-STAR unter der Kennung ULIT angebotene Version der Umweltliteraturdatenbank ULIDAT umfaßt gegenwärtig 76.000 Literaturhinweise. Das Angebot hat sich damit seit Beginn Des UMPLIS-Selbstwähldienstes im Januar 1984 mehr als verdoppelt.

Jeden Monat erhöht sich der Bestand etwa um mehr als ca. 1.000 aktuelle Hinweise auf relevante Fachliteratur, überwiegend mit inhaltlicher Kurzbeschreibung. 
Diese starke Expansion ist insbesondere auf die kontinuierliche und vollständige Erfassung der in der Bibliothek des Umweltbundesamtes eingehenden aktuellen umweltrelevanten Literatur sowie auf die weiterhin steigende Zahl umweltrelevanter Publikationen zurückzuführen. In der Regel können neuerscheinende Fachpublikationen bereits 4 bis 6 Wochen nach Erscheinen in der Datenbank abgefragt werden.

Die gute Qualität und Aktualität der Daten wird wesentlich durch den Einsatz des on-lineSystems DOKSIS erreicht, das u.a. die Eingabe von Dubletten verhindert und die Prüfung der bibliographischen Angaben (z.B. Zeitschriften- und Serientitel, Verlagsnamen, Institutionen) auf Einheitlichkeit vornimmt.

Als besonderer Schwerpunkt wurde der Bereich Umweltökonomie, zu dem etwa 7.000 Fachveröffentlichungen erfaßt wurden, bearbeitet. Demnächst wird im Erich-Schmidt-Verlag, Berlin, die aus diesem Datenbestand erstellte Bibliographie Umweltökonomie erscheinen.

Im Bereich des deutschsprachigen Umweltrechts ist die Erweiterung der Datenbank um 11.500 Literaturhinweise geplant, sodaß hier eine beachtliche Vollständigkeit erreicht werden kann. An diesem Teil von ULIDAT hat die Industrie sowie die ministeriale Ebene der Umweltverwaltung ein ganz erhebliches Interesse.

Die Übernahme von Fachliteratur über Forschungs- und Entwicklungsvorhaben der Umweltforschungsdatenbank in die Umweltliteraturdatenbank wurde fortgesetzt und eine Kopplung zwischen den Datenbanken vorgenommen.

Der Gesamtbestand der Umweltliteraturdokumentation, der außer der im Selbstwähldienst angebotenen Version ULIDAT auch einen ergänzenden Bestand überwiegend älterer Veröffentlichungen (ELIT) enthält, konnte auf ca. 153.000 Literaturhinweise erhöht werden.

1985 wurden in der Umweltliteraturdatenbank 2.262 Recherchen, davon 1.874 gegen Entgelt bei DATA-STAR, durchgeführt (siehe dazu auch Abb.4).

Die Pflege und Erweiterung der Umweltliteraturdatenbank (ULIDAT) erfolgt in enger Zusammenarbeit von Bund und Ländern. Die Bereiche Luft und Lärm werden bereits seit Jahren in Kooperation mit der Landesanstalt für Immissionsschutz des Landes Nordrhein-Westfalen (LIS) in Essen erarbeitet. 


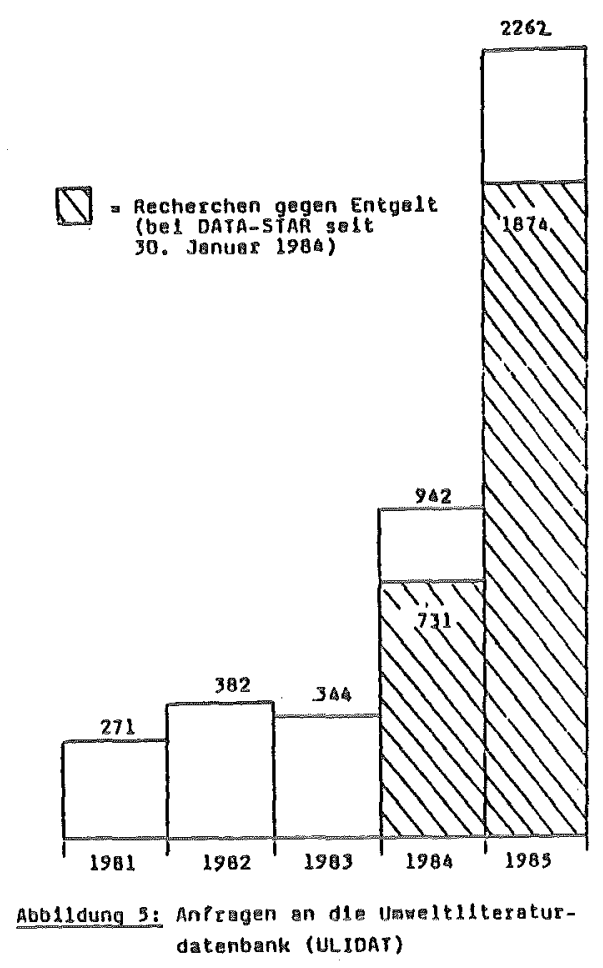

Im Bereich Ökologie wurde die Zusammenarbeit mit dem Bayerischen Landesamt für Umweltschutz zum Thema "Artenschutz in Bayern" aufgenommen. Mit Hessen wurde die Kooperation für den Bereich Bodenschutz vereinbart. Die Kooperation in weiteren Bereichen soll wesentlich ausgebaut werden. Dabei sollen Landesbehörden, u.a. in Baden-Württemberg und RheinlandPfalz sowie die Bundesforschungsanstalt für Naturschutz und Landschaftsökologie, einbezogen werden.

Der arbeitsteilige Input für die ULIDAT basiert auf den vom Bund/Länder-Arbeitskreis Umweltinformationssysteme (BLAK) beschlossenen "Materiellen Grundsätzen", die für verschiedene Stufen der Kooperation (Mindest-, Teil- oder Vollkooperation) in Abhängigkeit von den Leistungen der Partner, wie z.B. Lieferung der nicht im Buchhandel erhältlichen "Grauen Literatur", entsprechende Gegenleistungen, u.a. den entgeltfreien Zugriff auf die Datenbank, vorsehen.

Zur Erleichterung der Abfrage der Datenbanken im Umweltbundesamt sollen auch die weniger EDV-erfahrenen Benutzer mit den Möglichkeiten der Datenbanksuche vertraut gemacht werden. Deshalb ist ein Einfachdialog mit einer benutzerfreundlichen Abfragemöglichkeit entwickelt worden, dessen Ziel es ist, auch gelegentlichen Benutzern ohne EDV- und Datenbankkenntnisse zu schnellen und möglichst treffsicheren relevanten Informationen aus den Umweltliteratur- und -forschungsdatenbanken ULIDAT und UFORDAT zU verhelfen. Dabei wird angestrebt, den Zugriff so einfach zu gestalten, wie die Benutzung einer umfangreichen Handkartei in einer Bibliothek. Der Einfachdialog greift auf der Basis des Programmsystems 
ADABAS auf die unter dem Dokumentationssystem DOKSIS gespeicherten Datenbestände zu. Er wurde 1986 eingeführt und hat zu einer erheblichen Steigerung der Benutzung geführt. Dabei wird die Abfrage wesentlich durch das bei der inhaltlichen Erschließung verwendete Textanalysesystem ASTTAL unterstützt. Zur Steigerung der Trefferquote bei der Recherche sollen dabei alle relevanten Sachbegriffe, die zu jedem Dokument gespeichert sind, für die Suche herangezogen werden (kontrollierte und freie Deskriptoren).

Da die deutschsprachige Fachliteratur auf dem Umweltsektor in keiner ausländischen Datenbank verläßlich gespeichert ist, kommt der Vollständigkeit, Aktualität und Recherche-Treffsicherheit von ULIDAT eine große Bedeutung zu. Wie bei UFORDAT ist dieses Ziel jedoch nur näherungsweise zu erreichen und basiert vor allem auf der Mitarbeit vieler sachkundiger Partner. Da der Anteil der "Grauen Literatur” beim relevanten Gesamtbestand über fünf́zig Prozent́ beträgt, wird deutlich, wie schwierig die Sicherstellung einer verläßlichen Flächendeckung ist. Die umweltbezogene Fachliteratur ist aber auch in der "Weißen Literatur", z.B. Fachzeitschriften und Bücher) weit verstreut und keineswegs leicht zu erfassen.

Das von den Europäischen Gemeinschaften (EG) initiierte Dokumentationssystem des Europäischen Umweltrechts (ENLEX) wurde weiter ausgebaut. ENLEX arbeitet auf der Grundlage des bereits seit Jahren vom Umweltbundesamt finanziell unterstützten internationalen Environmental Law Informations System (ELIS) der International Union for Conservation of Nature and Natural Resources (IUCN).

Neben der Verläßlichkeit und Flächendeckung des Input und einer großen Benutzerfreundlichkeit des Mensch-Maschine-Dialoges (Einfachdialog mit verschiedenen Benutzerschalen) kommt der Treffsicherheit bei Recherchen die größte Bedeutung zu.

Nach meiner Einschätzung sind hier noch erhebliche Probleme zu bewältigen, bis der gelegentliche Benutzer zügig und sicher diejenigen Informationen aus den Datenbanken der wissenschaftlichen Dokumentation heraussuchen kann, die seinem Recherchethema entsprechen. Noch immer sind viele Wissenschaftler gezwungen, ihre eigene Dokumentation anzulegen und zu pflegen, um das Wichtige ihrer Fachdisziplin schnell verfügbar zu haben.

Ziel der Deskribierung muß es sein, daß die wichtigen und gebräuchlichen Fachbegriffe aller relevanten Benutzergruppen einer wissenschaftlichen Dokumentation vom System "verstanden" werden, also im System gespeichert sind. Dabei wären auch Eigenschaften der Assoziation und Ahnlichkeit neben den üblichen Beziehungen von Synonymen, Homonymen sowie Unter- und Oberbegriffe notwendig. Allein die Forderung nach Vollständigkeit aller relevanten Fachbegriffe stößt in große Dimensionen qualitativer und quantitativer Probleme vor. So sind auf dem 
Umweltsektor über 100.000 Chemikalien, einige 10.000 Pflanzen- und Tierarten, mindestens ebensoviele geographische Deskriptoren relevant, die zu den Fachdeskriptoren hinzukommen. Daß derartige Wortmengen für den gelegentlichen Benutzer unüberschaubar sind, braucht kaum erwähnt zu werden.

\section{Faktendatenbanken bei UMPLIS}

Neben einer zentralen Dokumentation hat das Umweltbundesamt gemäß dem Errichtungsgesetz den Auftrag, das Informationssystem zur Umweltplanung (des Bundes) aufzubauen. Wesentliche Voraussetzung dabei und Grundlage der Planung sind Daten über den Zustand der Umwelt in den Bereichen Luft, Lärm, Wasser, Boden, Abfallwirtschaft, Ökologie, Naturschutz, Landschaftspflege, usw.. Es geht also um die Umweltbeobachtung (Monotoring) und um verläßliche Daten über Struktur und Trends der Ursachen der Umweltbelastung und deren Gefahren.

Wesentlich ist weiterhin der Stand der Technik vor allem für die Vermeidung oder Beseitigung von Umweltbelastungen. Seit den Katastrophen von Tschernobyl und Sandoz wird immer deutlicher, daß Frühwarnsysteme und Schnellauskunftsysteme aufgebaut werden müssen, die nach dem neuesten Stand der Informationstechnik zu konzipieren sind.

Vor allem werden aber Daten zu chemischen Stoffen, deren Wirkungen auf die Umwelt sowie die Vermeidung und Abwehr von Umweltgefahren benötigt. Im folgenden werden die wichtigsten bei UMPLIS im Aufbau befindlichen Faktenbanken umrissen.

Besondere Bedeutung hat dabei künftig der weitere Ausbau der Zusammenarbeit von Bund und Ländern im Bereich der Faktendatenbanken, wie er sich im Bereich der Literatur- und Forschungsdokumentation bereits bewährt hat. Auch der Zusammenarbeit mit Industrie und Wirtschaft kommt eine große Bedeutung zu.

\subsection{INFUCHS, das System für chemische Stoffe}

Beim Informationssystem für Umweltchemikalien, Chemieanlagen und Störfälle - INFUCHS mit seinem Rahmensystem und den derzeit 5 Teilsystemen ist verstärkt die praktische Nutzung in den Vordergrund getreten. 
Für den Bereich der allgemein interessanten und zugänglichen technisch-wissenschaftlichen Daten liegt das Bestreben darin, den gespeicherten Datenbestand derart aufzubereiten, daß er für eine Nutzung Dritter besser geeignet ist. Weiterhin wird zu klären sein, in welcher organisatorischen Form der Datenbestand Dritten zugänglich gemacht werden kann und wie gegebenenfalls bereits bestehende Informationseinrichtungen genutzt werden können.

Gegenwärtig liegen die Arbeitsschwerpunkte beim Ausbau der Teilsysteme Chemikaliengesetz und Wasch- und Reinigungsmittel sowie bei der Verbesserung des Datenbestandes und der Erweiterung der bereits jetzt vorhandenen Zugriffsmöglichkeiten für UBA-externe Nutzer.

Ende 1985 wurde in diesem Rahmen u.a. für den Senator für Stadtentwicklung und Umweltschutz Berlin eine testweise Zugriffsmöglichkeit auf einen Teildatenbestand von INFUCHS geschaffen.

Zur Verbesserung des Datenbestandes von INFUCHS und für den Aufbau einer Unfallschnellauskunft wurde ein Kooperationsvertrag mit dem Springer-Verlag und dem Autor (G. Hommel) des bewährten und von der Benutzerseite her anerkannten "Handbuches der gefährlichen Güter" geschlossen.

Bei der Datenbank wassergefährdender Stoffe - DABAWAS -, die im Auftrag des Umweltbundesamtes vom Institut für Wasserforschung GmbH (IfW) in Dortmund betrieben wird, soll der bereinigte Datenbestand softwaremäßig in INFUCHS integriert werden. 1985 wurden 700 Auskünfte über Eigenschaften von wassergefährdenden Stoffen erteilt (1984: 509).

Seit November 1985 werden von IfW für diesen Auskunftdienst Entgelte erhoben, von denen Behörden mit unmittelbarem Rechtsstatus ausgenommen sind.

Durch Ressortbeschluß wurde ein Testlauf von INFUCHS für den Einsatz beim Vollzug des Chemikaliengesetzes beschlossen und im Oktober 1986 begonnen. Dabei arbeiten neben dem Umweltbundesamt vier weitere Bundesbehörden zusammen, um die Prüfung neuer Stoffe durchzuführen. Alle beteiligten Partner benutzen das zentral geführte System INFUCHS und allen stehen die neuesten Daten sofort nach Eingabe zur Verfügung.

Die für den Test von INFUCHS zU neuen Stoffen im Rahmen des Chemikaliengesetzes erforderlichen DV-Erweiterungsarbeiten sind inzwischen abgeschlossen.

Die INFUCHS-Teilsysteme Risiken in Chemieanlagen (RISCA) und Inhaltsstoffe von Wasch- und Reinigungsmitteln werden im Umweltbundesamt bei der täglichen Facharbeit genutzt und in 
diesem Zusammenhang wird der gespeicherte Datenbestand kontinuierlich fortgeschrieben und erweitert.

\subsection{System zur Beobachtung der Luftqualität LIMBA}

In der Luftimmissionsdatenbank LIMBA werden die Daten aus dem Meßstellennetz des Umweltbundesamtes und der Bundesländer gespeichert. Es sollen hiermit übergreifende Darstellungen und Auswertungen zur Immissionssituation und zudem Datenlieferungen an die EG aufgrund der Entscheidung des Rates der Europäischen Gemeinschaften vom 24. Juni 1982 zur "Einführung eines gegenseitigen Austausches von Information und Daten aus Meßnetzes und einzelnen Stationen zur Erfassung der Luftverschmutzung in den Mitgliedstaaten" durchgeführt werden.

Als Software für die Realisierung von LIMBA wurde vom Umweltbundesamt gemeinsam mit dem Land Bayern das System "Luftreinhaltung, Immission und Meteorologie SLIM" entwickelt. Für die grafische Darstellung von Datenbankinhalten und -auswertungen wird im Umweltbundesamt u.a. die Methodenbank Umwelt eingesetzt.

Der Bestand der Datenbank umfaßt gegenwärtig ca. 16,5 Mio. Meßwerte. Es handelt sich hierbei um Tagesmittelwerte für $\mathrm{SO}_{2}, \mathrm{NO}_{2}$ und Schwebstaub sowie um Stundenmittelwerte zu Ozon, $\mathrm{CO}_{2}$, Windrichtung und -geschwindigkeit, Temperatur und relative Feuchte.

Die Speicherung von Daten der Länder soll nun verstärkt werden. Das Land Bayern verwendet für seine Luftmeßdaten ebenfalls SLIM, sodaß hier keine Kompatibilitätsprobleme bestehen.

Von den Daten, die das Land Baden-Württemberg dem UBA lieferte, wurden die Werte $\mathrm{zu} \mathrm{SO}_{2}$ und Schwebstaub erfaßt.

Die Daten aus LIMBA werden in zunehmendem Maße für weiterführende Untersuchungen und Auswertungen zur Luftqualität herangezogen. Dies betrifft u.a. Auswertungen für "Daten zur Umwelt", flächendeckende Immissionsaussagen für die Bundesrepublik Deutschland oder statistische Zeitreihenanalysen.

\subsection{Emissionsursachenkataster EMUKAT}

Im Rahmen eines Forschungs- und Entwicklungvorhabens wurde im Umweltbundesamt ein Emissionsursachenkataster EMUKAT geschaffen. Es soll einen möglichst guten Überblick über die wichtigsten Emissionen in der Luft, deren geographische Verteilung in der Bundesrepublik 
Deutschland und die verursachenden Prozesse ergeben. Die flächendeckenden Emissionsdaten werden auf der Grundlage verfügbarer Daten zu Emissionsursachen rechnerisch ermittelt.

Zunächst werden die Werte für Schwefeldioxid $\left(\mathrm{SO}_{2}\right)$ und Stickoxide $\left(\mathrm{NO}_{\mathrm{x}}\right)$ berechnet. Für die Berechnung der gesamten Emissionen wird unterschieden nach:

- Punktquellen für Großemittenten (z.B. Kraftwerke und Industrieanlagen)

- Linienquellen (z.B. Emissionen durch Verkehr auf Autobahnen)

- Flächenquellen (Zusammenfassen verschiedener "kleiner” Emissionen)

Für die Emittentengruppen Kraftwerke und Industrie wurden mittlerweile Angaben zu ca. 1.500 größeren Anlagen gespeichert, die den überwiegenden Teil des entsprechenden Energieverbrauches ausmachen. Für die Emittentengruppen Haushalte/Kleinverbraucher sind ca. 350 Datensätze mit kreisbezogenen Aussagen gespeichert. Für den Bereich Verkehr liegen ca. 1.000 Autobahnabschnitte als Linienquellen vor.

EMUKAT-Daten wurden geliefert für das deutsch-holländische Forschungsprojekt PHOXA, das Programm EMEP der Genfer Luftreinhaltekonvention für Europa, die Emissionskataster der EG und der OECD, das Projekt Energie und Umwelt der Bundesforschungsanstalt für Landeskunde und Raumordnung sowie die Umweltberichte "Daten zur Umwelt 1984" des Umweltbundesamtes.

Für die Fortschreibung des „Daten zur Umwelt”, die Anfang 1987 erscheinen werden, wurde im Rahmen eines Pilotvorhabens eine Aktualisierung des Datenmaterials für das Bezugsjahr 1984 vorgesehen.

\subsection{Abfallwirtschaftsdatenbank AWIDAT}

Die 2. Auflage des Handbuches der Verwerterbetriebe ist im Erich Schmidt Verlag, Berlin, erschienen. Es handelt sich hierbei um einen aufbereiteten Datenbestand aus der Verwerterdatei der Abfallwirtschaftsdatenbank (AWIDAT). Das Handbuch der Verwerterbetriebe ist in zwei Hauptteile gegliedert und wird aus den Datenbestand von AWIDAT erzeugt:

\section{- Hauptteil I: Verwerterbetriebe}

Er enthält das Verzeichnis der aufgenommenen Verwerterbetriebe, gegliedert nach 17 Gruppen. Zu jedem Verwerterbetrieb sind neben der Anschrift ausführliche Informationen zu Art und Herkunft der angenommenen Rückstände, Annahmehinweise sowie Angaben zu den Verwertungs- und Behandlungsverfahren gespeichert. 
- Hauptteil II: Roh-/Altstoffhändler

Hier sind Informationen zu Händlern bzw. Abfallbeseitigungsbetrieben gespeichert, die in den Bereichen Stahl- und Legierungsschrott, Altmetalle, Altpapier/Altglas, Alttextilien/Hadern, Altkunststoff/Altgummi, Autoverwertung, Tierische Rohstoffe etc. tätig sind.

Das Handbuch enthält vier Register, die von verschiedenen Fragestellungen ausgehend einen gezielten Zugriff auf die Informationen des Hauptteils I ermöglichen.

Die Daten von AWIDAT sollen eventuell über einen Host allgemein zugreifbar werden. Der Bedarf an Daten zur Abfallwirtschaft ist erheblich und zwar sowohl für Sonderabfälle, von denen häufig Gefahren ausgehen, als auch für die politischen Ziele der Abfallvermeidung und Wiederverwendung (Recycling). Daher werden im UBA Überlegungen angestellt, derartige Datenbestände on-line abfragbar aufzubereiten.

\subsection{Gewässerkundliche Datenbank HYDABA}

Die umweltrelevante gewässerkundliche Datenbank HYDABA hat mittlerweile umfangreiches Datenmaterial zur Wasserqualität von Oberflächengewässern gespeichert. Vorwiegend für Auswertungen und Aussagen des Bundesministers für Umwelt, Naturschutz und Reaktorsicherheit, des Umweltbundesamtes und der internationalen Gewässerschutzkommission sowie die „Daten zur Umwelt" sind z.B. 1985400 Auskünfte aus der Datenbank erteilt worden.

HYDABA wird bei der Bundesanstalt für Gewässerkunde in Koblenz geführt und ist in das Gesamtkonzept von UMPLIS integriert. Es wird das System ADABAS im Zusammenwirken mit mehreren Benutzerprogrammen verwendet. Der grafischen Datenverarbeitung kommt dabei eine große Bedeutung zu.

\subsection{Bibliotheksdatenbank BIBLIODAT}

Die Bibliothek ist zu einer wesentlichen Informationsquelle nicht nur für das Umweltbundesamt, sondern ebenfalls für eine ständig wachsende Zahl von deutschen und ausländischen Benutzern geworden (1985: 3.000 externe Besucher).

Die wissenschaftliche Bibliothek des Umweltbundesamtes hat ihren Bestand auf 56.100 Bände erhöht; zusätzlich werden laufend 490 Zeitschriften gehalten. Die Microfiches-Sammlung einschließlich der Forschungsberichte der Environmental Protection Agency (EPA) der USA umfaßt z.Zt. etwa 110.000 Einheiten. 
Durch die enge Zusammenarbeit mit der Literatur- und Forschungsdokumentation wurde die Schlagwortvergabe für den Bibliotheksbestand fortgeführt. Da bisher kein Schlagwortkatalog existiert, wurde mit dem Aufbau auf der Grundlage der ULIDAT-Deskriptoren begonnen. Der direkte Zugriff auf die Bibliotheksdatenbank BIBLIODAT, die bisher vor allem für die Katalogerstellung Anwendung findet, wird mit der Umstellung auf das Datenbanksystem DOKSIS wesentlich erleichtert.

Für die weitere Entwicklung der UBA-Bibliothek sollen Ausleihe, Beschaffung und vor allem der Informationsservice für das Umweltbundesamt durch einen verstärkten EDV-Einsatz verbessert werden. Langfristig ist der Ausbau zu einer bedeutenden Umwelt-Fachbibliothek der deutsch. sprachigen Literatur im Verbund mit anderen Bibliotheken angestrebt.

Mitte 1986 wurde eine verbesserte Test-Datenbank BIBLIODAT für die on-line-Abfrage (Einfachdialog) zur Benutzung freigegeben. Darin sind die ab 1984 erfaßten Bände und Berichte enthalten, die auch in ULIDAT gespeichert sind. Es wurde in Kombination mit der Microfiche-Sammlung eine Infothek eröffnet, die als "EDV-gestützter Facharbeitsplatz Bibliothek" einzustufen ist.

Schon nach kurzer Zeit ist der Andrang so groß geworden, daß ein zweiter Arbeitsplatz eingerichtet werden muß.

\subsection{Grafische Umweltdatenbasis GRAFU}

Die Möglichkeiten des Grafiksystems der Methodenbank Umwelt GRAFU zur Speicherung und Darstellung von Meßergebnissen, Emissionen und Belastungen in Form von Bildern und Karten wurde umfangreich genutzt; GRAFU wird gemäß dem erheblichen Benutzerbedarf stürmisch ausgebaut.

1985 sind rund 9.000 grafische Darstellungen abgefragt worden. Besondere Aufmerksamkeit fanden auch 1986 wieder die jährlich herausgegebenen Waldschadenskarten des Umweltbundesamtes. Die erstellten Grafiken wurden 1985 in 405.000 gedruckten Exemplaren verbreitet und im Fernsehen sowie den Lesern von Zeitungen und Zeitschriften mit einer Gesamtauflage von 6 Millionen zugänglich gemacht.

Die erreichte neue Qualität der Datenauswertung basiert auf der Möglichkeit der grafischen Datenverarbeitung, umfangreiche Daten, wie sie im Umweltschutz vorliegen, in ihren räumlichen Auswirkungen und deren gegenseitige Beeinflußung zu verarbeiten und darzustellen. Die 
grafische Datenverarbeitung hat sich als ideale Kommunikationsschnittstelle zu Faktendaten erwiesen:

- Trends, Muster, kritische Zustände werden erkennbar, einprägsam, vergleichbar, bewertbar.

- Der lokale und räumliche Bezug von Umweltgeschehen wird visualisierbar.

Neben der Erstellung einzelner Grafiken spielt GRAFU eine zentrale Rolle bei der Umweltberichterstattung. Der Bericht "Daten zur Umwelt 1984" /5/ ist bereits in einer dritten Auflage mit insgesamt über 17.000 Exemplaren erschienen.

Anfang 1987 wird eine erweiterte und aktualisierte Fassung der Öffentlichkeit vorgestellt. Bei der Neuerstellung der Daten zur Umwelt hat sich gezeigt, daß der Einsatz von GRAFU nicht nur eine wichtige Auswertfunktion von Faktendaten übernimmt, sondern auch umfangreiche Daten aufnimmt und an Datenbanken übergibt.

So wird im Rahmen diese Projektes in enger Zusammenarbeit mit anderen Bundesbehörden von diesem die thematisch umfassendste Umweltdatensammlung erstellt, die weite Bereiche der Anforderungen des sog. Grunddatenkataloges abgedeckt.

Die so gewonnenen Daten werden durch GRAFU verarbeitet, gespeichert und in Grafiken umgesetzt. Zu anderen raumbezogenen Informationssytemen werden zur Zeit Schnittstellen geschaffen, um bereits vorhandene Geometrien in GRAFU zu übernehmen und weiterzuverarbeiten, so z.B. zum Grafiksystem der Bundesforschungsanstalt für Naturschutz und Landschaftsökologie (BFANL).

Im Rahmen der Umweltbeobachtung durch Satelliten (Remote Sensing) werden 1986 flächendeckende Daten für den Bereich der Bundesrepublik ausgewertet und für GRAFU verfügbar sein. Mit Hilfe des neuen Grafik-Arbeitsplatzes 9732-1 der Firma SIEMENS können diese Rasterdateien verarbeitet werden, sodaß Verknüpfungen mit bereits vorhandenen räumlichen Umweltaussagen durchgeführt werden können.

Mit dem Umweltatlas Berlin wurde erstmalig eine umfangreiche Kartierung eines urbanen Ökosystems mit Hilfe der Methodenbank Umwelt durchgeführt und der Öffentlichkeit zur Verfügung gestellt. Es ist beabsichtigt, den Umweltatlas Berlin so zu erweitern, daß er auch als Instrument zur Erarbeitung von Planungsentscheidungen eingesetzt werden kann. 


\section{Neue Aktivitäten hoher Priorität}

4.1 Smog-Frühwarnsystem für die Bundesrepublik Deutschland

Das Smog-Frühwarnsystem soll vor allem für diejenigen Luftschadstoffe eingesetzt werden, die von außerhalb in die Bundesrepublik transportiert werden. Dieser Anteil ist nach neuen Untersuchungen teilweise auf bis zu 70\% und darüber einzuschätzen. Bekanntlich treten Smog-Episoden im allgemeinen in den Wintermonaten auf; es sei besonders auf die Smog-Situation vom Januar 1985 erinnert.

Das Frühwarnsystem für advehierten Smog gliedert sich grundsätzlich in fünf Komponenten:

1. Meßnetz in der Bundesrepublik Deutschland

Das Meßnetz wird sich aus verschiedenen Teilnetzen zusammensetzen, da die einzelnen Bundesländer und der Bund derartige Netze betreiben. Für den Smog sind vor allem Meßstationen in der Nähe der Grenze von Bedeutung, während in den Ballungsgebieten nur wenige ausgewählte Meßpunkte verwendet werden sollen.

2. Meßflugzeug

Bei Bedarf und entsprechender Wetterlage sollen Meßflugzeuge gestartet werden, die in verschiedenen Höhenschichten entsprechende Messungen vornehmen. Die Messungen sollen an die Zentrale am Boden übermittelt werden, um dann in dem Rechner mit den anderen Daten verbunden zu werden.

3. Datenübertragungsnetz

Die verschiedenen, von den Bundesländern betriebenen, Meßnetze werden mit einem zusätzlichen "Schnittstellenrechner" auszustatten sein, von dem die Daten dann an die Zentrale nach Berlin zum Umweltbundesamt übermittelt werden. Gegenwärtig wird der PC IBM-AT3 getestet, um Klarheit darüber zu gewinnen, ob dieses Gerät als Schnittstellenrechner geeignet ist.

4. Zentrale Speicherung und Verarbeitung der Daten Im Umweltbundesamt wird ein Luftrechner in Betrieb genommen, der eine temporäre SmogDatenbank aufnehmen wird. In diese Datenbank wird über das Meßnetz die Gesamtmenge der Daten übermittelt werden und zu einer Smog-Karte der Bundesrepublik Deutschland zusammengesetzt. Durch den grafischen Einfachdialog sollen einfache Abfragen über verschiedene Phasen der Smog-Situation ermöglicht werden. Später sollen mit Hilfe eines verlässlichen Prognose-Modells Vorhersagen für die wahrscheinliche Smog-Entwicklung ermöglicht werden. 
5. Nutzungssystem der Daten

Die verschiedenen übermittelten und berechneten Daten zur Smog-Episode sollen von allen Teilnehmern genutzt werden können. Hierfür wird das übertragungsnetz, das für den Daten-Input Verwendung findet, ebenfalls für die Nutzung und den Zugriff eingesetzt werden. Im Umweltbundesamt selbst sollen leistungsfähige Arbeitsplatzrechner (workstations) eingesetzt werden, während bei den Ländern die Schnittstellenrechner mit entsprechender Grafik auszurüsten sind, um auch hier einen grafischen Einfachdialog zu ermöglichen. Dabei sollen verschiedene Smog-Situationen in Kartenform und mit Fenstertechnik abfragbar sein. Daneben sind zeitliche Entwicklungen für verschiedene Punkte in Form eines Polygonzuges darzustellen.

\subsection{Waldschadensinformationssytem}

In der Bundesrepublik Deutschland bestehen an den verschiedensten Stellen wertvolle Daten über Waldschäden, die in einem System zusammengefaßt werden sollen. Ähnlich wie beim Smog-Frühwarnsystem sollen die Daten in einer zentralen Datenbank zusammengefaßt werden und mit geeigneten Benutzeroberflächen abfragbar sein. Hierfür kommen die Systemkomponenten wie beim Smog-Frühwarndienst zur Anwendung.

Insbesondere ist an einen grafischen Einfachdialog mit Hilfe von PCs gedacht. Die Besonderheit bei diesem System liegt darin, daß sehr verschiedenartige Datenbestände zentral archiviert und geordnet abgelegt werden müssen und anschließend in einem geführten einfach zu beherrschenden Dialog für die verschiedensten Aufgaben nutżbar gemacht werden sollen. Es versteht sich von selbst, daß dabei auf die Luftdaten zurückgegriffen werden muß (z.B. Luftimmissionsdatenbank LIMBA).

\subsection{Informationssytem für Schäden an Baudenkmälern und Kulturgütern}

Neben den Waldschäden treten die durch verschiedene Umweltverschmutzungen hervorgerufenen Schäden an Kulturgütern (z.B. Denkmäler, Bauwerke und dergleichen) immer mehr in den Vordergrund. Es ist z.B. zu beobachten, daß in den letzten Jahrzehnten der Steinzerfall an derartigen Kulturgütern rapide zugenommen hat, sodaß größere Schädigungen in einem Jahrzehnt entstanden sind, als in vielen Jahrhunderten früher. Damit gehen Kulturwerte von unschätzbarem Wert verloren. 
Als wesentlicher Schritt für Gegenmaßnahmen soll eine umfassende Dokumentation der Schäden und Gegenmaßnahmen aufgebaut werden. Dabei sollen zunächst die bestehenden Datenbanken für die Fachliteratur (ULIDAT) und die Forschungsvorhaben (UFORDAT) genutzt werden, um auch die entsprechenden Daten zu diesem Bereich aufzunehmen. Dabei ist der Thesaurus entsprechend auszuarbeiten und zu erweitern.

Darüberhinaus soll vor allem eine Dokumentation der eingeleiteten Gegenmaßnahmen und Schutzmittel, die an den verschiedenen Kulturdenkmälern in Deutschland vorgenommen wurden, aufgebaut werden, um aufgrund der dort gemachten Erfahrungen praktische Hinweise für erfolgreiche Maßnahmen zu gewinnen. Wesentlich in diesem Teilsystem wird auch die Verwendung der grafischen Datenverarbeitung sein, denn es soll ein Schadensatlas der Bundesrepublik Deutschland mit EDV-Unterstützung erstellt werden.

Von großer Bedeutung ist auch in diesem System, daß es gelingt, praxisgerechte und leicht $2 u$ bedienende Benutzeroberflächen zu schaffen. Daher sollen die Erfahrungen vom Einfachdialog auf dieses System übertragen werden. Es wird vor allem auch um einen grafischen Einfachdialog gehen, bei dem nicht nur kartografische Darstellungen sondern auch grafische Dokumentationen der Schäden selbst zu realisieren sind. Dieses System soll mit dem Informationszentrum Raum und Bau (IRB) zusammen entwickelt und aufgebaut werden.

\subsection{EDV-Einsatz für die Forschungsplanung im Umweltbundesamt}

Die Datenbank der Forschungsvorhaben UFORDAT wird gegenwärtig so erweitert, daß der

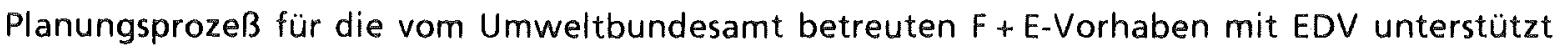
wird. Dazu werden die geplanten Vorhaben in eine Planungsdatei übernommen, die für die verschiedenen Abstimmungsprozesse und Prioritätensetzungen geeignet ist. Insbesondere ist hier ein Abgleich zwischen dem Umweltbundesamt und dem Bundesminister für Umwelt, Naturschutz und Reaktorsicherheit erforderlich. Nach der Bewilligung werden die Daten einerseits für den allgemeinen Zugriff in die UFORDAT übernommen und zum anderen für interne Verwaltungs- und Planungsprozesse im Umweltbundesamt bereitgehalten. Dabei sind laufend Daten zum Haushaltsvollzug einzugeben: Sollbuchungen, Istbuchungen, Terminverschiebungen, Aufstockungen und dergleichen. Dieses System enthält etwa 150 zusätzliche Felder und wurde in relativ kurzer Zeit (im wesentlichen in ca. zwei Monaten) realisiert. Dieses ist dadurch möglich, daß die Dateivereinbarung, der Pflegedialog und der Abfragedialog sowie der Listenausdruck ohne größere Neuprogrammierungen ermöglicht wurde, weil das System DOKSIS durch Tabellen- und Parameterverarbeitung für diese neue Aufgabe lediglich angepaßt werden mußte. Die 
bereits vorhandenen Ausgabelisten mit FIDAS werden über eine Schnittstelle weiterhin verwendet.

Es hat sich gezeigt, daß bei der Listenverarbeitung der Report-Generator IREG von FIDAS nach wie vor von erheblichem Nutzen ist.

Durch die Kombination mit den durch den Einfachdialog (System EMDIAL) ermöglichten on-lineBenutzeroberflächen, ergibt sich ein Leistungsspektrum der Nutzung und Datenausgabe, das nach unseren Erfahrungen allen Anforderungen gerecht wird.

\subsection{Schnellauskunft für gefährliche Stoffe und gefährliche Güter}

Bereits seit einiger Zeit wird im Rahmen von INFUCHS intensiv an einer Datenbank für die Schnellauskunft über gefährliche Stoffe und Güter gearbeitet. Nachdem die Daten aus dem "Handbuch gefährlicher Güter" von Hommel übernommen worden sind, kann nunmehr die praktische Benutzung in der Bundesrepublik Deutschland vorbereitet werden. Dazu wurden einfache Abfragemasken geschaffen, die für die erste halbe Stunde am Einsatzort (erster Angriff) und für die folgende Einsatzleitung geeignet sind. Es geht dabei zunächst um Sofortmaßnahmen und den Schutz der am Unfallort beteiligten Personen (Atemschutz, Löschvorgaben, Gefährlichkeitsklassifizierung und dergleichen). Wie der Brand bei Sandoz gezeigt hat, sind bei derartigen Katastrophen und Störfällen erhebliche Schädigungen für die Umwelt zu befürchten. Daher soll INFUCHS auch für die folgenden Schutzmaßnahmen eingesetzt werden können.

4.6 Integriertes EDV-System für Fachliteratur, Forschungsvorhaben und Bibliothek

Die drei Dokumentationsbereiche Literatur, $F+E-V o r h a b e n$ und Bibliothek sind eng miteinander verknüpft, und bilden für den wissenschaftlichen Benutzer eine Einheit. Beispielsweise werden Veröffentlichungen und der Abschlußbericht zu einem Forschungsvorhaben nachgefragt, wenn ein Vorhaben abgeschlossen ist. Der Benutzer will dann im allgemeinen nicht nur die Zielsetzung des Forschungsvorhabens wissen, sondern möchte auch auf die Ergebnisse zugreifen, wie sie in Veröffentlichungen und Ergebnisberichten niedergelegt sind. Erfahrungen im Umweltbundesamt gehen dahin, daß pro F + E-Vorhaben zwei bis drei Veröffentlichungen publiziert werden.

In einem Bibliothekskatalog wird im allgemeinen nur die selbständige Literatur, wie Monographien, Sammelbände, Forschungsberichte, Dissertationen und ähnliches katalogisiert und über Deskriptoren zugreifbar gemacht. Diese Daten sind aber auch in den Literaturdatenbanken enthalten, wobei einige spezielle Angaben für die Bibliothek entfallen können (Anzahl der 
Bände, Auflage, Fortsetzungsbände, Serien und dergleichen). Über die Daten der Bibliothek hinaus sind in der ULIDAT dann die Daten über Einzelveröffentlichungen aus Sammelbänden, Zeitschriften, usw. abgespeichert.

Es versteht sich von selbst, daß Doppelarbeiten bei der Datenpflege vermieden werden können, wenn die einzelnen Datenbanken einen gemeinsamen integrierten Grunddatenbestand verwenden. Für den Benutzer werden daraus verschiedene Aspekte ausgewählt und in entsprechenden Benutzerschalen zusammengefaßt. Dabei geht es unter anderem um den Pflegedialog in Bibliothek, Fachliteratur und F + E-Vorhaben. Die Daten der Bibliothek (BIBLIODAT) sollen nach den RAK-Regeln erfaßt werden.

Bislang bestanden bei den Benutzern der Bibliothek zum Teil erhebliche Akzeptanzprobleme für on-line-Recherchen. Der gute alte Karteikartenkatalog wurde im allgemeinen gegenüber EDVSystemen bevorzugt. Umfangreiche Bandkataloge in Form von Computerausdrucken haben dabei die Akzeptanz des EDV-Einsatzes nicht gerade gefördert. Durch des Einsatz des Einfachdialoges bei UMPLIS konnte hier jedoch in einem Testlauf nachgewiesen werden, daß auch ungeübte Benutzer, die als Einsteiger oder gelegentliche Benutzer in die Bibliothek kommen, problemlos mit dem System arbeiten können.

Wesentlich ist neben dem Nachweis der Literatur durch die entsprechenden Datenbanken, daß leicht auf die Texte zugegriffen werden kann. Aus diesem Grund werden bei UMPLIS von allen Veröffentlichungen, die nicht in Zeitschriften der Bibliothek vorhanden sind, Kopien gefertigt und in einer sogenannten Separata-Sammlung abgelegt. Über eine Verweisnummer ist es dann möglich, die Hinweise aus den Datenbanken für den Zugriff auf diese Kopien zu verwenden.

\section{Hardware des Umweltbundesamtes}

1985 ist das Rechenzentrum in neue Räume umgezogen. Um die gestiegenen Kapazitätsanforderungen befriedigen zu können, wurde gleichzeitig fast die gesamte Hardware ausgetauscht. Als Zentraleinheit steht nunmehr ein Rechner des Typs 7.570-B der Firma Siemens zur Verfügung. Während der regulären Arbeitszeit ist die Anlage sehr stark belastet. Die Rechenanlage soll daher noch in mehreren Stufen dem ständig steigenden Bedarf angepaßt werden. Die Speicherkapazität auf Magnetplatten wurde auf über 6 Milliarden Zeichen angehoben. 
Im Rahmen eines Forschungsvorhabens wurde ein DEC-Rechner VAX 11/780 installiert und mit dem UBA-Rechner für die grafische Darstellung chemischer Strukturen von Stoffen gekoppelt. Darauf basierend werden Áhnlichkeiten mit Strukturen von chemischen Substanzen, deren Umweltverträglichkeit bereits bekannt ist, abgefragt.

Durch den Anschluß des Siemens-Rechners an das Berliner Rechner-Netz BERNET und an das Rechenzentrum des Bundesgesundheitsamtes bestehen Zugriffsmöglichkeiten auf Datenbestände und Rechenkapazitäten von Dritten.

Wegen des gestiegenen Bedarfs soll die Anzahl der installierten Datensichtgeräte erhöht werden. Außerdem wurde ein neuer Prozeßrechner (PDP-11/73) in der Pilotstation Frankfurt für die Daten des Luftmeßnetzes in Betrieb genommen.

Damit wird die Pilotstation in die Lage versetzt, ihre Funktion als Zenrale für das Luftmeßnetz des Umweltbundesamtes auch im Bereich der Meßdatenverarbeitung zu erfüllen.

Im Dezember 1986 wird ein neuer Rechner (Siemens $7570 \mathrm{C}$ ) in Betrieb genommen, der die gesamten Luftdatenbanken zusammenfaßt und vor allem für das Smog-Frühwarnsystem eingesetzt werden soll.

Messungen bei den erzielten Antwortzeiten haben ergeben, daß Werte von weit über 20 Sekunden keine Seltenheit sind. Bei den Antwortzeiten ergeben sich typische Verlaufskurven während eines Arbeitstages: Am Vormittag gegen elf Uhr ergibt sich ein erstes, am Nachmittag ein zweites (meist höheres) Maximum. Arbeitspsychologische Erfahrungswerte für die zumutbaren Antwortzeiten eines on-line-5ystems liegen bei maximal drei bis fünf sekunden. Gerade beim geführten einfachen Mensch-Maśchine-Dialog wird die angestrebte Akzeptanz benutzerfreundlicher Systemoberflächen durch unzumutbare Antwortzeiten zunichte gemacht. Aber auch bei Input- und Update-Vorgängen sind zu langsame Reaktionszeiten des Systems außerordentlich negativ zu bewerten; sie führen zu erhöhten Kosten für die Datenbankpflege oder machen die notwendige Aktualität unmöglich.

Daher muß die Leistungsfähigkeit der Hardware vor allem bei der Prozessorleistung der CPU, bei der Größe der Arbeitsspeicher und bei den Platten-Kanälen wesentlich verbessert werden.

Faktendatenbanken bedürfen grafischer Darstellungen, die einfach zu bedienen und flexibel konzipiert sind. Daher sollen neben leistungsfähigen workstations in zunehmendem Maße PCs mit Grafikeigenschaften mit den Hauptrechnern vernetzt betrieben werden. Es ist zu hoffen, daß bei den PCs Farbmonitore mit einer Auflösung von ca. 1 Millionen Bildpunkten angeboten werden, sodaß kartografische Umweltdaten mit der nötigen Abbildungsschärfe on-linę dargestellt werden können (grafischer Einfachdialog). 


\section{6. Überlequngen zu einem Softwarekonzept von UMPLIS}

Wegen der großen Bandbreite von Softwareanforderungen bei UMPLIS (Wissenschaftliche Dokumentation, Faktendatenbanken, Grafik, Planungssyteme, usw.) wurde von Anfang an versucht, universelle Standard-Softwarepakete einzusetzen. So wurden ab 1976 die Systeme FIDAS ( das vom Autor vor ca. fünfzehn Jahren zusammen mit der Gesellschaft für Mathematik und Datenverarbeitung/GMD entwickelt wurde) GOLEM und ADABAS eingesetzt. Dabei ist ADABAS bekanntlich ein Datenbank-Grundsystem, das erst im Zusammenspiel mit Anwenderprogrammen (z.B. in COBOL, PL1 oder NATURAL) zur Anwendungsreife kommen kann.

Vor allem wegen Problemen für Input, Update und Dateikoppelung aber auch wegen der Akzeptanzprobleme für gelegentliche Benutzer beim IN-HAUS-System mußten Aufgaben von GOLEM auf FIDAS und schließlich auf ADABAS verlagert werden. Es entstand das integrierte Programmsystem DOKSIS für Datenbankaufbau und -pflege, EMDIAL (Einfacher MenschMaschine-Dialog) und ASTTAL für die Thesauruspflege und Indexierungshilfe. Es ist in PL 1 ausgelegt und weist über 100.000 Programmzeilen auf. Kürzlich haben sich auch zwei andere Bundesbehörden zum Einsatz dieses Systems entschieden.

Für die Verwaltung sehr großer Datenbestände vor allem aus Meßnetzen (Umwelt-Monitoring) mit 50 Millionen Meßwerten und mehr wurde ebenfalls auf der Basis von ADABAS zusammen mit dem Land Bayern das System SLIM entwickelt. Bei UMPLIS wird es - teilweise in Kombination mit DOKSIS/EMDIAL - für die Luftdatenbanken LIMBA und EMUKAT sowie für das im Aufbau befindliche Smog-Frühwarnsystem verwendet. Auch SLIM ist in PL1 programmiert und umfaßt ca. 140.000 Programmzeilen.

Ein drittes großes Programmpaket wurde für das universelle Chemikalieninformationssytem INFUCHS aufgebaut; es wurde in COBOL und NATURAL ausgeführt und umfaßt ebenfalls mehr als 100.000 Programmzeilen.

FIDAS wird nach wie vor häufig für die Listenaufbereitung sowie für schnell zu realisierende ADHOC-Arbeiten (z.B. Datenumsetzungen und Erzeugen von Arbeitsdateien) verwendet.

Beim Grafiksystem fiel die Wahl auf SICAD, das u.a. für GRAFU, den Umweltatlas Berlin und die Daten zur Umwelt verwendet wird. Durch den Einsatz von farbigen hochauflösenden Monitoren ist dieses System für Umweltkarten (z.B. Waldschadenskarten) als sehr leistungsfähig einzustufen. Es wird in Kombination mit dem PLOT-System von CALCOM verwendet. 
Welche Aufgaben stehen nun für die Zukunft im Vordergrund?

Um den Rahmen dieser Darstellung nicht́ zu sprengen, sollen die zukünftigen Entwicklungslinien in zehn Zielbereichen abgesteckt werden:

1) Bei den für geographische Bezüge ausgelegten Systemen SLIM/DOKSIS soll eine Integration mit SICAD hergestellt werden.

2) Grafikfähige PCs sollen mit den Zentralsystemen unter ADABAS vernetzt werden und den grafischen Einfachdialog für geografische Karten, darstellende Statistik, Managementgrafik usw. ermöglichen.

3) Durch die Vernetzung mit PCs soll die Fenstertechnik bei den Suchhilfen mit Registern eingesetzt werden.

4) Chemische Strukturen sollen darstellbar und recherchierbar gestaltet werden.

5) Die Softwarepakete sollen so weit integriert und abgerundet werden, daß neue Anwendungen bei Faktendatenbanken und Dokumentation weitgehend ohne Neuprogrammierung (einschließlich NATURAL) ermöglicht werden.

6) Die Methodenbank Umwelt soll weiter ausgebaut werden.

7) Die Textverarbeitung mit "dezentraler Intelligenz" soll mit den Zentralsystemen (z.B. DOKSIS) integriert werden.

8) Der Einfachdialog soll weiter ausgebaut werden, um thematische Recherchen unabhängig von der Unterteilung in Datenbanken zu ermöglichen. Durch Kopplungspfade sollen die Datenbestände so vernetzt werden, daß eine integrierte Umwelt-Wissensbasis entsteht.

9) Ausgehend von Datenbanken, Methodenbank und Einfachdialog 'sollen Prototypen für Expertensysteme entwickelt werden.

10) Durch den Einsatz von workstations sollen leistungsfähige Facharbeitsplätze geschaffen werden. 


\section{Literaturverzeichnis}

/1/ Seggelke,J.;

Das Informations- und Dokumentationssystem Umwelt - UMPLIS -

In: Gesellschaft für Mathematik und Datenverarbeitung und Umweltbundesamt (Hrsg.),

Datenverarbeitung auf dem Umweltsektor - Vorträge einer gemeinsamen Veranstaltung,

München, Wien 1978, S.101-132

12/ Kunz,W., Reuter,W. , Rittel (Hrsg.);

UMPLIS, Entwicklung eines Umwelt-Planungs-Informationssystems

Fallstudie Informationssyteme 8, Grundlagen und Praxis der Informationswissenschaften,

München, New York, London, Paris 1980, $300 \mathrm{~S}$

13/ Page,B., Seggelke,J.;

UMPLIS - Ein umfassendes Informationssystem für den Umweltschutz

In: Informatik im Umweltschutz. Anwendungen und Perspektiven, Prof. Dr. Ing. Page (Hrsg.),

München, Wien 1986, S.178-192

14/ Fachinformation, Programm der Bundesregierung

Der Bundesminister für Forschung und Technologie (Hrsg.), Bonn 1985, $127 \mathrm{~S}$

15/ Daten zur Umwelt 1984

Umweltbundesamt (Hrsg.), Berlin 1984, 3995 
Ein Schalenmodell für den Einfachdialog verschiedener Benutzergruppen beim Umweltinformationssystem UMPLIS

\author{
J. Seggelke \\ Umweltbundesamt Berlin \\ Bismarckplatz 1, 1000 Berlin
}

\title{
Zusammenfassung
}

Auf der Grundlage des Datenbankverwaltungssystems ADABAS wurde eine besonders benutzerfreundliche Systemoberfläche entwickelt, die sich in mehrere Schalen mit unterschiedlicher Schwierigkeit und Leistungsfähigkeit (Mächtigkeit) gliedert. Die praktische Erprobung dieses einfachen Mensch-Maschine-Dialoges (Systembezeichnung EMDIAL), der zusammen mit dem Anwendersystem DOKSIS für die wissenschaftliche Dokumentation durchgeführt wurde, ergab eine erhebliche Steigerung der Nutzung, besonders für gelegentliche Benutzer (Steigerung um über 500\%). Es ist zu hoffen, daß durch EMDIAL ein wesentlicher Teil der Hemmnisse zur Benutzung der im Umweltbundesamt aufgebauten Datenbanken beseitigt und die Akzeptanz erhöht wird. Die Anwendung für Faktendatenbanken und Erweiterung um grafische Komponenten sind geplant.

\section{Gliederung}

1. Ausqangslage und Probleme

2. Definition der Benutzergruppen

3. Benutzeranforderungen des Einfachdialoges

3.1 Allgemeines

3.2 Einfachdialog und kommandoorientierte Retrievalsprachen

3.3 Benutzerschale 1 (Einsteiger)

3.4 Benutzerschale 2 (gelegentliche Benutzer)

3.5 Benutzerschale 3 (häufige Benutzer)

4. Softwarelösung des Schalenmodells

5 Nutzung der Umweltliteraturdatenbank ULIDAT mit dem Einfachdialog EMDIAL

6. Weiterentwicklung des Einfachdialoges und Ausblick

7. Literaturverzeichnis 


\section{Ausgangslage und Probleme}

Im Rahmen des Umweltplanungs- und Informationssystems UMPLIS /1/, /2/ wurden neben verschiedenen Faktendatenbanken die Literaturdatenbank ULIDAT und die Datenbank für Umweltforschungsvorhaben und -institutionen (UFORDAT) aufgebaut. Beide Datenbanken werden über den Host DATA-STAR für den allgemeinen Zugriff gegen Entgelt angeboten. Demnächst sollen sie auch bei INKAISTN aufgelegt werden, sodaß ein weiterer Benutzerkreis erschlossen wird.

Der bisherige Verlauf der Benutzerstatistik des Selbstwähldienstes bei DATA-STAR zeigt, daß sich beide Datenbanken am Markt gut behaupten können und positiv in bezug auf Flächendeckung und Aktualität eingeschätzt werden. Es muß jedoch darauf hingewiesen werden, daß der online-Markt bislang nur eine sehr begrenzte Anzahl von Benutzern aufweist (die Schätzung für die Bundesrepublik Deutschland liegt bei insgesamt ca. 1.000 bis 1.500 aktiven Benutzern). Gegenwärtig greifen Endbenutzer, die gelegentlich oder nur fallweise Recherchenbedarf haben, kaum direkt auf die Datenbank zu. Hier liegt ein großes mögliches Nutzungspotential für Datenbanken, das bislang nicht erschlossen worden ist.

Eine wesentliche Benutzergruppe von UMPLIS ist das Umweltbundesamt selbst, das die Bundesregierung in allen Fragen des Umweltschutzes wissenschaftlich berät. Da das Umweltbundesamt nicht über wesentliche eigene Forschungskapazitäten und Labore verfügt, kommt der Funktion "Informationsverarbeitung" eine sehr große Bedeutung zu. Die wissenschaftlich arbeitenden Kollegen des Umweltbundesamtes sind daher ein bedeutender Prüfstein für die Praktikabilität, Nützlichkeit und Akzeptanz der durch UMPLIS bereitgestellten Informationen.

Die Datenbanken ULIDAT und UFORDAT waren in der Vergangenheit im Umweltbundesamt nur mit dem Softwarepaket GOLEM zugreifbar. Der Erfolg war dabei mäßig. Nachdem in den letzten Jahren ca. 100 mögliche und meist auch interessierte Endbenutzer geschult wurden, haben etwa 15 Benutzer nachfolgende Versuche in der Abfrage von Datenbanken angestellt. Nur etwa 5 benutzten die Datenbank regelmäßig bei der laufenden Arbeit, aber 3 hiervon besitzen bereits fundierte EDV-Erfahrungen und sind daher nicht als typische Endbenutzer einzustufen.

Diskussionen in dem Interessenkreis der Datenbankanwender und -hersteller (IDAH) ergaben die verblüffende Übereinstimmung dieser nicht gerade positiven Erfahrungen. Trotz aller bisherigen Versuche ist es demnach nicht gelungen, Endbenutzer, die gelegentlich oder fallweise Datenbanken einsetzen wollen, zu gewinnen. Bislang sind fast ausschließlich „Profis" als on-lineBenutzer tätig, die oft für die eigentlichen Endbenutzer Recherchen durchführen und sich auf Datenbankabfragesprachen und Datenbanken spezialisiert haben. 
Es ergibt sich folgende Schlußfolgerung:

Bei vorhandenem Bedarf nach Informationen und gutem Datenbankangebot (Flächendeckung, Aktualität und Treffsicherheit) reicht die Benutzerfreundlichkeit der vorhandenen Abfragesprachen für den gelegentlichen Benutzer noch immer nicht aus. Es fehlt eine Form des Einfachdialoges zwischen Mensch und Maschine, der nicht wesentlich höhere Anforderungen stellt als die Benutzung einer größeren Handkartei oder eines Bibliothekskataloges. Die mangelnde Akzeptanz bei vielen Endbenutzern muß in der nächsten Zeit überwunden werden, um das reichhaltige Informationsangebot aus vorhandenen oder noch entstehenden Datenbanken fruchtbar werden zu lassen.

Um die aufgezeigten Probleme zu lösen, wurde bei UMPLIS das nachfolgend beschriebene Schalenmodell entwickelt, dessen Einfachdialog hoffentlich einen Schritt in die richtige Richtung ergeben wird. Erste Ergebnisse bei praktischen Einsatz können als erfolgsversprechend eingeschätzt werden.

\section{Definition der Benutzergruppen}

Um die Voraussetzung für die Konzeption eines Einfachdialoges zu schaffen, sollte bei den bislang üblichen Dokumentationshilfen angeknüpft werden. Herkömmliche umfangreiche Dokumentationen werden in Form von Handkarteien, Handbüchern oder Nachschlagekatalogen geführt. Grundsätzlich kann hierzu gesagt werden, daß kaum spezielles instrumentelles Wissen bzw. eine entsprechend vorauslaufende Schulung erforderlich sind, um diese Dokumentationshilfsmittel sinnvoll zu benutzen. Die verwendeten Schlagworte sind meist für die jeweiligen Fachleute selbsterklärend, sodaß es keines gesonderten Thesaurus bedarf und es insbesondere nicht erforderlich ist, die Schlagworte im vorhinein aus dem Thesaurus auszusuchen.

Typisches Beispiel einer derartigen umfangreichen Handkartei ist das System der Bibliothekskataloge: Es besteht meist aus einem Autorenkatalog, einem Schlagwort- oder Sachkatalog, einem Zeitschriftenkatalog und einer Systematik der Signatur, die gleichzeitig für das Aufstellen der Bände in den Regalen Verwendung findet.

Ein gebundener Katalog zum Nachschlagen - wie etwa der Umweltforschungskatalog des Umweltbundesamtes - besteht aus der Auflistung der F+E.-Vorhaben (als Hauptteil I), der Auflistung der forschenden Institutionen (als Hauptteil II), sowie folgenden Registern: 
- Schlagwortregister

- Personenregister

- Geographisches Register

- Ortsregister der durchführenden Institutionen

- Register der finanzierenden Stellen

Die aufgeführten Formen der Dokumentation werden von Endbenutzern im allgemeinen ohne Schwierigkeiten gehandhabt. Bei diesen Endbenutzern lassen sich im wesentlichen drei Gruppen unterscheiden:

- "Einsteiger" und Einmalbenutzer (etwa 1 bis 2 x jährlich)

- gelegentliche Benutzer (z.B. 3 bis $10 \times$ pro Jahr)

- häufige oder professionelle Benutzer.

Durch diese drei unterscheidbaren Benutzergruppen sind drei Schalen für einen MenschMaschine-Dialog definierbar. Bei UMPLIS wird deshalb von einem Drei-Schalen-Modell für die Benutzung in Form eines Einfachdialoges zwischen Mensch und Maschine ausgegangen.

\section{Benutzeranforderungen des Einfachdialoges}

3.1 Allgemeines

Ziel des Einfachdialoges ist es, mit einem absoluten Minimum an EDV-Wissen die Datenbanken benutzen zu können. Als Minimum wird angenommen, daß eine Schreibmaschinentastatur und die Schreibmarke eines EDV-Sichtgerätes sowie die Taste für die Datenübertragung an den Rechner bedient werden können. Diese Fähigkeiten können in der Tat in knapp einer halben Stunde erlernt werden und sind unumgänglich, um überhaupt mit einem EDV-gestützten System arbeiten zu können.

Im übrigen soll beim Einfachdialog die Fragebogentechnik mit Hilfe der Bildschirme realisiert werden: Diese wurde in der empirischen Sozialforschung und der Psychologie entwickelt und hat sich weitgehend bewährt. Die Form der Fragebogentechnik mit elektronischen Instrumenten wird auch als geführter Dialog bezeichnet.

Als Benutzergruppe wird festgelegt, wer mit fachlichen Handbüchern, Katalogen und Handkarteien sinnvoll arbeiten kann oder will. Der Arbeitsprozeß soll dabei soweit es geht an diesen herkömmlichen Instrumenten ausgerichtet sein. Auf diese Weise wird der notwendige Lernaufwand minimiert und die Akzeptanzschwelle so weit es geht abgesenkt. 
Bei einer arbeitspsychologischen Analyse der Benutzung eines Dokumentationskataloges wie etwa des Umweltforschungskataloges oder der Benutzung einer umfangreichen Kartei lassen analytisch folgende einzelne Arbeitsschritte unterscheiden:

- Einstieg in die Dokumentation (meist über ein Schlagwort),

- Heraussuchen der Dokumentationseinheiten, die dem Schlagwort zugeordnet sind,

- Einschätzen der Relevanz der gesichteten Dokumentationseinheiten,

- Feinselektion nach fachlichen Gesichtspunkten für die bereits ausgewählte Menge der Dokumentationseinheiten,

- Entscheidung über die endgültige Menge der ausgewählten Dokumentationseinheiten.

Neben den o.g. wichtigsten Funktionen laufen jedoch eine ganze Anzahl verschiedenster fachlicher Bearbeitungsschritte $a b$, ohne daß sich der Benutzer vermutlich darüber immer im klaren ist. Beispielsweise werden die Güte der Schlagwortvergabe, die Menge der Schlagworte, die evtl. im chronologischen Ablauf veränderte Gewohnheit der Schlagwortvergabe u.ä. mitabgeschätzt. Die gesichteten Dokumentationseinheiten werden weiterhin nach Relevanz zum thematischen Fragenbereich eingestuft. Gleichzeitig wird jedoch auch die Frage selbst, d.h. der thematische Gegenstand des Benutzerwunsches, präzisiert. Es ist nämlich davon auszugehen, daß das Thema der Recherche eventuell nicht in der nötigen Präzision festgelegt worden ist, wie dies eigentlich der Fall sein sollte.

Zum einen wird manchmal zu speziell recherchiert, sodaß im Laufe der Arbeit mit der Handkartei das Thema auszuweiten ist. Dieses geht integriert mit anderen Vorgängen durch das Sichten und fachliche Gewichten der gefundenen Dokumentationseinheiten vor sich. Zum anderen wird ein zu breit angelegtes Recherchethema durch die Fülle der gefundenen Dokumentationseinheiten einzugrenzen sein, um eine überschaubare und bearbeitbare Menge von Dokumentationseinheiten zu erlangen.

Beim Übergang zu einer EDV-gestützten umfangreichen Dokumentation treten eine Reihe von neuen Problemen hinzu, die bei einer Handkartei nicht zu beobachten sind, da der Umfang der Dokumentation und die Anzahl der zu vergebenden Schlagworte meistens wesentlich größer sind. Daraus ergeben sich Folgeprobleme:

Der Selektionsprozeß muß schärfer vorgenommen werden, um die Fülle der gefundenen Informationen zu bewältigen. Dies bedeutet z.B. auch, daß die Vergabe von Deskriptoren vor Einspeicherung in die Datenbank präziser und systematischer vollzogen werden muß als bei einer Handkartei.

Ein weiteres Problem bei vorhandenen Abfragesprachen von Datenbanken liegt darin, daß der Recherche- und Selektionsprozeß von der Sichtung der Dokumentationseinheiten getrennt ist. 
Bei den kommandoorientierten Abfragesprachen, wie sie gegenwärtig z.B. bei den HOSTZentren gebräuchlich sind, dominiert im allgemeinen das Ziel einer möglichst kurzen Anschaltzeit. Auf diese Weise ist es dem Benutzer oder Profi (Schale $3 \mathrm{im}$ vorgestellten Modell) möglich, Recherchen in kürzestmöglicher Zeit durchzuführen. Genau diese Anforderung verhindert jedoch, daß der Einsteiger und gelegentliche Benutzer (Schale 1 und 2) einfach mit dem System umgehen kann. Der Benutzer dieser beiden Schalen benötigt erheblich mehr Hilfen am Datensichtgerät und erheblich mehr Überlegungszeit, um mit dem für ihn ungebräuchlichen Instrument umgehen zu können. Dies ist im übrigen auch bei Handkatalogen ähnlich, denn bevor ein Benutzer einen umfangreichen Bibliothekskatalog für seine Aufgabe nutzen kann, wird er eine Orientierungs- und Lernzeit aufbringen müssen.

Aus dem o.g. ergibt sich demnach eine andere Zielsetzung bei gelegentlichen Benutzern als bei Profis: eine Bearbeitungszeit von $\frac{1}{2}$ bis 1 Stunde wird durchaus als normal anzusehen sein. Dabei ist es erforderlich, auf möglichst viele bekannte Arbeitsprozesse abzustellen, psychologische Barrieren vor dem Instrument EDV abzubauen (z.B. durch eine Ermutigung in der Einführung) und die zu erlernenden Schritte für den EDV-Einsatz auf das absolute Minimum zu beschränken.

Charakteristisch für EDV-gestützte Dokumentationen sind die logischen Kombinationen der Einzelelemente des Recherchethemas. Verwendet werden hier das logische UND, ODER sowie UND NICHT. Diese Boolsche Algebra ist bei der Benutzung herkömmlicher Dokumentationen völlig ungewohnt. Sie mag zwar implizit bei Benutzern mitspielen, ist aber explizit kaum bewußt. Daher ist die Boolsche Logik für Einsteiger und gelegentliche Benutzer verwirrend und erschwert den Umgang mit dem EDV-System. Zu fragen ist daher, ob bei der Konstruktion eines Einfachdialoges darauf verzichtet werden kann.

Intensive Diskussionen und praktische Tests ergaben, daß bei der Verwendung von Deskriptoren sowohl das logische UND als auch das logische ODER erforderlich sind, um auf eine überschaubare Menge von Dokumentationseinheiten zu kommen. Würde man nur das logische ODER zulassen, wäre im allgemeinen - selbst in der einfachsten Form des Dialoges - für den Einsteiger die Menge der ausgeworfenen Dokumentationseinheiten zu groß. Würde man nur das logische UND zulassen, wäre wiederum die Selektion zu eng, sodaß breitere Themengebiete nicht sinnvoll bearbeitet werden können. Es ist daher eine besondere Schwierigkeit für die Schale 1 und 2 des Einfachdialoges, eine implizite Form des UND und ODER zu finden, die von der überwiegenden Anzahl der Benutzer richtig verwendet wird, ohne daß die explizite Kenntnis der Boolschen Algebra vorausgesetzt werden muß. Unseres Wissens ist das bisher keiner Abfragesprache im Mensch-Maschine-Dialog gelungen. 
3.2 Einfachdialog und kommandoorientierte Retrievalsprachen

Bislang werden Retrievalsprachen grundsätzlich in zwei Formen angeboten:

1. Kommandoorientierte Sprache

2. Geführter Dialog

Bei kommandoorientierten Abfragesprachen hat jede wesentliche Funktion einen Kommandonamen (z.B. FIND, ADES, SEARCH, FINDE oder dergleichen für das Suchkommando) sowie einen genau festgelegte Syntax für die einzelnen Elemente der Suchfrage.

Ähnlich werden Kommandos für die Anzeige der gefundenen Dokumentationseinheiten, für das Auslösen des Druckes, für den Wechsel der Datenbank, für das Kennzeichnen der Deskriptoren in Registern und dergleichen verwendet. Der Nachteil dieser Kommandosprachen besteht darin, daß der Benutzer nach einer genauen syntaktischen Vorschrift seine Angaben auf einem weitgehend leeren Bildschirm einschreiben muß, und daß er die Abfragesprache weitgehend beherrschen muß. Erfahrungsgemäß ist dieses für den seltenen Benutzer aber fast unmöglich, da die Spezifika einer EDV-Syntax im allgemeinen nur bei permanenter Benutzung geläufig und daher bei seltener Benutzung unzumutbar sind.

Beim geführten Dialog wird demgegenüber grundsätzlich in Form eines Frage- und Antwortspiels die Menge der für die EDV notwendigen Angaben festgelegt, und es sind sog. Masken, also EDV-geführte Formulare, auszufüllen. Diese Form des Dialoges hat den Vorteil, daß auch der Anfänger wesentliche Hilfen am Sichtgerät vorfindet und bei richtiger Konstruktion eines derartigen Mensch-Maschine-Dialoges praktisch kaum Vorkenntnisse für die Bearbeitung benötigt. Die Nachteile liegen jedoch darin, daß der geübte Benutzer zu viele unnötige Arbeitsschritte durchlaufen muß und nicht die nötige Effizienz erreicht werden kann. Außerdem kann sich ein Gefühl der Schwerfälligkeit einstellen, das insbesondere anspruchsvolle Benutzer demotiviert. Anzumerken ist, daß häufig auch bei geführten Dialogen sehr genaue Vorschriften für die Art der Eingabe zu beachten sind und daß häufig die Fehlerkommentare schwer verständlich und alles andere als eine Hilfe sind.

Bei der Konstruktion eines einfachen Mensch-Maschine-Dialoges EMDIAL für die genannten Benutzerschalen 1 bis 3 besteht daher grundsätzlich die Forderung, daß mit zunehmenden erlernten Fähigkeiten der Benutzer die Effizienz der Benutzung gesteigert werden muß, sodaß der kundige Benutzer schnellere Wege der Problemlösung einschlagen kann als der Anfänger und gelegentliche Benutzer. Im Dialog ist damit die Forderung nach Variabilität und Schnelligkeit des Dialoges gegeben. 
Bei allen Kommentaren und Hinweisen ist auf größte Verständlichkeit zu achten, die sich nicht an EDV-erfahrenen Benutzern zu orientieren hat, sondern an Benutzern, die mit Handbüchern und Karteien erfolgreich fachlich gearbeitet haben. Am besten ist ein Psychologe hinzuzuziehen, der Akzeptanzprobleme einzuschätzen weiß.

Beim Einfachdialog von UMPLIS wurde nicht auf eine Benutzergruppe abgezielt, die bislang ohne Dokumentationen und Handbücher ausgekommen ist. Eine derartige Benutzeranforderung muß als überzogen bezeichnet werden, da hier kein fachliches Interesse und keine fachliche Erfahrung an der Dokumentation vorausgesetzt werden kann.

Der Einfachdialog hat entsprechend dem bisher Ausgeführten folgenden grundsätzlichen Ablauf:

- Auswahl der gewünschten Datenbank

- Suchen in der Datenbank nach den gewünschten Informationen

- Ansehen der gefundenen Informationen am Bildschirm

- Drucken der Informationen aus der Datenbank.

Der Standardablauf entsteht dadurch, daß einfach die Taste "Datenübertragung" gedrückt wird und ein linear vorwärtsgeführter Dialog abläuft. Sprungmöglichkeiten müssen möglich sein, um Verbesserungen durchführen zu können. Dieses gilt insbesondere für die Veränderung des Suchthemas aufgrund der eingegebenen Deskriptoren.

\subsection{Benutzerschale 1 (Einsteiger)}

Auf der Benutzerschale 1 werden nach Auswahl der Datenbank folgende Einzelangaben für das Suchthema für erforderlich gehalten:

- Umweltbereich

- Deskriptoren mit impliziter UND- und ODER-Verknüpfung

- geographischer Bezug

- Autor

- Erscheinungsjahr.

Die Formulierung am Bildschirm für die implizite UND- und ODER-Gestaltung sieht folgendermaßen aus:

- Hauptthema (z. B. Waldschaden, Korrosion)

- Thematische Einengung (z.B. $\mathrm{SO}_{2}$ )

- weitere Einengung (z. B. Rauchgas). 
Um die gefundenen Informationen anzusehen, gibt es eine Kurzdarstellung, die im Falle der Literaturdokumentation nur aus Autor, Titel und Publikationsjahr besteht. Wesentlich hierbei ist, daß man die Dokumentationseinheit auswählen kann, die von Interesse sind, sodaß alle übrigen wegfallen.

\subsection{Benutzerschale 2 (gelegentliche Benutzer)}

Die Schale 2 baut auf der Schale 1 auf und erweitert den Umfang der Suchmöglichkeiten:

- 2. Autor

- Institution (z.B. Herausgeber eines Forschungsberichtes)

- Titel der Publikation

- Fundstelle oder die Datenbanknummer.

Eine wesentliche Erweiterung gegenüber der Schale 1 soll durch umfangreiche Suchhilfen, z.B. in Form von Registern, vom System ermöglicht werden. Hierbei geht es vor allem um die Umweltklassifikation und um eine thematisch, eine alphabetisch und eine hierarchisch geordnete Liste der Deskriptoren (verschiedene Formen des Umweltthesaurus). Außerdem ist ein geographisches Register sowie ein Autorenregister von Bedeutung. Es ist durch einfaches Ankreuzen möglich, nach Einsichtnahme in die Register die entsprechenden Register zu markieren und in den Suchbildschirm zu übertragen.

\subsection{Benutzerschale 3 (Häufige Benutzer)}

Die Benutzerschale 3 entspricht im wesentlichen dem, was heute kommandoorientierte Sprachen leisten, wie z.B. GOLEM, DIRS, MESSENGER, BRS-Dialog usw.. Jedoch soll beim Einfachdialog die Form des geführten Ablaufes am Sichtgerät beibehalten werden. Daher ist auf der Schale 3 der gesamte Umfang der Boolschen Algebra mit beliebiger Verknüpfung von UND und ODER anzubieten. Es sollten Suchthemen (SDI) gespeichert werden und später wieder aktiviert werden können. Die Rechercheergebnisse aus der Datenbank werden im einzelnen detalliert aufgegliedert nach "Treffern" in der Datenbank, hierarchischen Beziehungen der Deskriptoren und logischer Verknüpfung. Weiter wird zwischen kontrollierten und freien Deskriptoren unterschieden. 


\section{Softwarelösung des Schalenmodells}

Als Grundsoftware wird das Datenbanksystem ADABAS eingesetzt, das mehrere Datenbanken verwaltet und weitgehend die Eigenschaften eines relationalen Datenbanksystems realisiert (Multi-file-Konzept). Aufbauend auf ADABAS ist das System DOKSIS entwickelt worden, das vor allem für eine effiziente Dateneingabe und Datenpflege geeignet ist. Der gesamte geführte Dialog für die Abfrage wird mit dem Programmsystem EMDIAL (Einfacher Mensch-MaschineDialog) realisiert, das auf die einzelnen Dateien zugreift. Die 3 Benutzerschalen bauen aufeinander auf und sind aufwärts kompatibel gestaltet. Eine leistungsfähige Benutzerverwaltung, in der Anschaltzeiten, Zugriffe und Datenbanken und verwendete Deskriptoren aufgezeichnet werden, rundet das Softwarepaket ab. Diese statistischen Kennzahlen sind on-line abfragbar.

Der gesamte Ablauf als Folge der einzelnen Bildschirme ist in Tabellenform in PL 1 programmiert worden, sodaß grundsätzlich für neue Bildschirmmasken keine Programmänderungen notwendig sind. Die Gestaltung einer neuen Bildschirmmaske für einen bestimmte Benutzergruppe, die durch einen Benutzereintrag im System festgelegt ist, erfordert erfahrungsgemäß für den Systemverwalter nicht mehr als einige Arbeitsstunden. Auf diese Weise lassen sich alle Benutzerwünsche im Rahmen des Leistungsumfanges des Systems schnell realisieren, insbesondere werden benutzertypische Formulierungen leicht einzubauen sein. Dieses wiederum kann wesentlich zur Verbesserung der Akzeptanz beitragen.

Für die Thesaurusverwaltung und Unterstützung der Deskribierung wird ein besonderes Programmpaket (ASTTAL) im Einfachdialog eingesetzt:

Mit diesem Programm wird der Umweltthesaurus gepflegt und eine halbautomatische Indexierung durchgeführt. ASTTAL weist ähnliche Grundzüge wie das Programmpaket PASSAT auf. Es führt die in den eingegebenen Texten vorhandenen Fachbegriffe auf ihre Grundform zurück, erkennt Synonyme und kann in gewissem Umfang auch zusammengesetzte Begriffe in seine Bestandteile auflösen. Es wird im Einfachdialog ebenfalls zur Analyse des Suchthemas eingesetzt, sodaß auch Suchanfragen in Umgangssprache eingegeben werden können. 


\section{Nutzung der Umweltliteraturdatenbank ULIDAT mit dem Einfachdialog EMDIAL}

Nach verschiedenen Verbesserungen der Benutzeroberfläche wurde zusammen mit einer Testgruppe von ca. 20 Teilnehmern der Einfachdialog konzipiert.Für die Einführung von neuen Benutzern wird ca. eine halbe bis eine Stunde benötigt. Dabei kann die Schale 1 und die Schale 2 im einzelnen erläutert und praktisch vorgeführt werden. Wichtig ist, daß die Benutzer selbst gemäß ihren Aufgaben- und Fragestellungen mit dem System arbeiten können. Die Schale 3 wird nur im groben innerhalb der kurzen Einführungszeit behandelt, da sie die Boolsche Algebra und ausgefeilte Verknüpfungsmöglichkeiten der Deskriptoren zum Inhalt hat und weiterhin die Abspeicherung von Suchfragen als SDI ermöglicht.

Das Ergebnis des Einfachdialoges ist in Abb.1 wiedergegeben. Danach hat sich die IN-HAUSNutzung im Umweltbundesamt für die Umweltliteraturdatenbank ULIDAT bis zum Beginn im Juli 1986 auf niedrigem Niveau bewegt. Es wurde das System GOLEM eingesetzt, wobei die Anzahl der monatlichen Recherchen etwa zwischen 20 und 50 variierte. Wie der Abbildung zu entnehmen ist, hat sich die Nutzung der Datenbank nach Einführung des Einfachdialoges sprunghaft erhöht, sodaß die Anzahl der Recherchen pro Monat deutlich über einer Steigerung von $500 \%$ liegt. Es ist nicht ausgeschlossen, daß Steigerungen des Einfachdialoges von $1.000 \%$ gegenüber der kommandoorientierten Abfragesprache GOLEM erreicht werden können.

Zusammenfassend kann gesagt werden, daß sich die Erwartungen für die Nutzungssteigerung des Einfachdialoges erfüllt haben bzw. daß die zu erhoffenden Ergebnisse bei weitem übertroffen worden sind. Es scheint, daß das Ziel der Nutzung für ungeübte Benutzer voll erreicht werden konnte. Dies hat auch eine Vielzahl von Gesprächen mit nicht EDV-geschulten Interessenten ergeben.

Während des bisherigen Einsatzes des Einfachdialoges war die Antwortzeit durch Überlastung des Rechners relativ hoch, sodaß Antwortzeiten von über 30 Sekunden vorgekommen sind. Es braucht kaum erwähnt zu werden, daß dieses völlig unzumutbar ist. Daher muß angestrebt werden; daß durchschnittlich Recherchen unterhalb von 5 Sekunden bleiben und nur in Ausnahmefällen (bei komplizierten Recherchen oder aufwendigen Registern) Antwortzeiten von max. 10 Sekunden vorkommen.

Aufgrund der ermutigenden Ergebnisse ist es demnach wahrscheinlich, daß Endbenutzer selbst mit derartigen Systemen im Einfachdialog arbeiten können. Damit würde sich für eine Vielzahl von Datenbanken eine völlig neue Möglichkeit der Nutzung eröffnen. Es wäre denkbar, daß derartige Einfachdialoge nicht nur für IN-HAUS-Systeme verwendet werden, sondern auch im Hostbetrieb für jedermann gegen Entgelt zugänglich sind. Dabei wäre darauf zu achten, daß die 


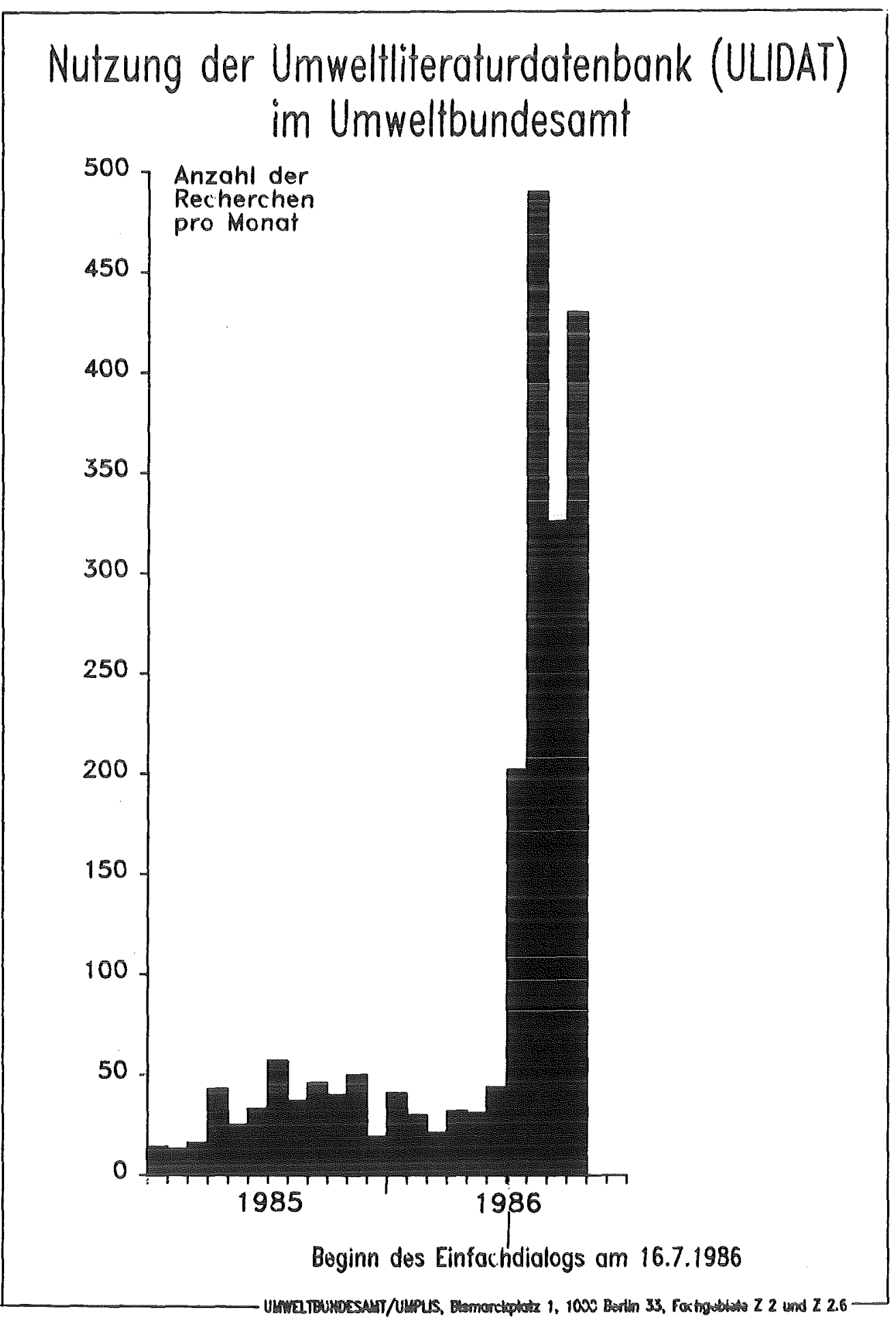

Abbildung 1

Anschaltzeit nicht wesentliche Grundlage der Berechnung der Entgelte ist, weil dadurch der Anfänger und gelegentliche Benutzer keine Zeit hätte, um im geführten Dialog Überlegungen anzustellen. Im übrigen haben Tests im Umweltbundesamt ergeben, daß die Antwortzeiten von GOLEM und dem Einfachdialog für geübte Benutzer durchaus gleichwertig sind, wenn beim Einfachdialog die Schale 3 mit allen Möglichkeiten der Deskriptorverknüpfung gewählt wird. 
Damit ist auch die häufig geäußerte Meinung widerlegt, daß geführte Dialoge zwangsläufig erheblich größere Anschaltzeiten und Bearbeitungsdauern benötigen als kommandoorientierte Retrievalsprachen.

\section{Weiterentwicklung des Einfachdialoges und Ausblick}

Durch die sprunghaft gestiegenen Benutzungszahlen bei UMPLIS dürfte der Nachweis gelungen sein, daß durch den Einfachdialog mit verschiedenen Benutzerschalen die bisherigen Hemmnisse bei Datenbanken in einem erheblichen Ausmaß abgebaut werden können. Welche Weiterentwicklungen sind nun für die Zukunft vordringlich?

Ein Datenbanksysten wie ADABAS, das die Eigenschaften eines relationellen Systems aufweist, bietet die Möglichkeiten, flexible Kopplungen zwischen Datenbanken zu realisieren. Bei UMPLIS ist z.B. die Kopplung zwischen den F+E-Vorhaben (UFORDAT) und der Fachliteratur (ULIDAT) wichtig, um die zu einem bestimmten Vorhaben gehörende Literatur (Forschungsergebnisse, Ergebnisberichte) aufzufinden.

Die Möglichkeit der Datenbank-Kopplung wurde daher beim Einfachdialog testweise realisiert und soll demnächst für die allgemeine Benutzung freigegeben werden.

Bei der Auswertung des Einfachdialoges auf Faktendatenbanken bei UMPLIS spielen grafische Informationen (z.B. Waldschadenskarten, Luftgüterkarten, usw.) eine wesentliche Rolle. Daher soll zunächst für das Smog-Frühwarnsystem der Bundesrepublik eine Erweiterung zum grafischen Einfachdialog realisiert werden. Dabei sollten Personal Computer (PC) als preiswerte Grafik-Terminals verwendet werden. Im übrigen sollen die wichtigen Dialogschritte wie Datenbankauswahl, Suchbildschirm, Kurzübersicht, usw., sowie die Form des Dialoges beibehalten werden. Weitere Ziele sind flexiblere Druckgestaltung und Sortierung, Ausbau der SDIEigenschaften, Kopplungen innerhalb einer Datenbank (z.B. Zitate bei der Literaturdatenbank), Berechnungen bei Faktendatenbanken, usw. Beim Einsatz von PCs als Terminals kann weiterhin durch die Fenstertechnik die Arbeit mit Registern benutzerfreundlicher gestaltet werden. 
7. Literaturverzeichnis

/1/ Seggelke,J.;

Das Informations- und Dokumentationssystem Umwelt - UMPLIS -

In: Gesellschaft für Mathematik und Datenverarbeitung und Umweltbundesamt (Hrsg.),

Datenverarbeitung auf dem Umweltsektor - Vorträge einer gemeinsamen Veranstaltung,

München, Wien 1978, S.101-132

12/ Page,B., Seggelke,J.;

UMPLIS - Ein umfassendes Informationssystem für den Umweltschutz,

In: Informatik im Umweltschutz. Anwendungen und Perspektiven, Prof. Dr. Ing. Page (Hrsg.),

München, Wien 1986, S.178-192 
Verzeichnisgesieuerte Faktendatenbanksysteme am Beispiel des Informationssystems für Umweltchemikalien, Chemieanlagen und Störfälle (INFUCHS) M. Stopp Umweltbundesamt Berlin Bismarckplatz 1, 1000 Berlin 33

\section{Zusammenfassung}

Die Hauptprobleme bei der Realisierung von Datenbanksystemen bestehen im zeitlichen Verzug zwischen der Aufnahme der Anforderungen und deren Realisierung und bei den sich fortlaufend ändernden und nie endgültig festlegbaren Benutzerwünschen. Damit sich diese Kluft nicht immer weiter vertieft, sind neue Wege beim Entwerfen und Realisieren erforderlich. Am Beispiel der Chemiekaliendatenbank INFUCHS wird gezeigt, wie man durch die Verwendung von Verzeichnissen (Data Dictionary) problemoffene Faktendatenbanksysteme realisieren kann.

\section{Gliederung}

1. Einführung

2. Probleme und Lösungsansätze

3. Ausblick

4. Literatur 


\section{Einführung}

Anforderungen an Faktendatenbanksysteme IMIE 85/ aus dem Umweltbereich zeichnen sich im Vergleich zu vielen kommerziellen systemen durch ein hohes Maß an Komplexität in den Aufgabenstellungen und in den Datenstrukturen aus. Auch die Mengen der zu verarbeitenden Daten können erhebliche Ausmaße annehmen, insbesondere bei kontinuierlichen Messungen von Umweltgütedaten, z.B. aus den Bereichen Luft und Wasser

Durch die Zielsetzung, die Datenbanken nicht nur verwaltungsintern zu nutzen, sondern soweit möglich, auch darüberhinaus zugänglich zu machen, ergibt sich ein sehr heterogener Benutzerkreis, der unterschiedlichste Anforderungen an die Bedienbarkeit der Systeme stellt.

Soll mit den Datenbanken auch der Vollzug von gesetzlichen Regelungen unterstützt werden, ergeben sich außerdem die unterschiedlichsten Zugriffs- und Schutzprobleme.

Die Vielzahl der geschilderten Probleme und der schnelle wissenschaftliche bzw. technische Fortschritt aus dem sich fortlaufend Änderungen der Benutzerwünsche ergeben, erfordern neue Wege beim Entwerfen und Realisieren von Datenbanksystemen, um einerseits den Zeitraum zwischen Anforderung und Realisierung nicht zu groß werden zu lassen, und andererseits neuen Anforderungen ohne jeweils erneuten großen Entwicklungsaufwand gewachsen zu sein.

Die Verwendung von verzeichnisgesteuerten Systemen scheint ein Weg, um problemoffene Faktendatenbanksysteme zu realisieren.

Die Probleme und Lösungen werden am Beispiel der Chemiekaliendatenbank INFUCHS (Informationssystem für Umweltchemikalien, Chemieanlagen und Störfälle) aufgezeigt, bei dem alle geschilderten Probleme auftreten /STO 83/, ISTO 86/. INFUCHS ist die Stoffdatenbank des Umweltbundesamtes im Rahmen des Informations- und Dokumentationssystems Umwelt (UMPLIS) /UMP 85/.

\section{Probleme und Lösungsansätze}

Der Wunsch bzw. die Notwendigkeit für den Aufbau einer Datenbank entsteht in den meisten Fällen dadurch, daß an einer Stelle eines Unternehmens oder einer Behörde Daten anfallen bzw. benötigt werden, bei denen z. B. die Menge so groß ist oder die Strukturen der Daten so komplex sind oder mit denen viele Anwender gleichzeitig arbeiten müssen, daß der Einsatz der Datenverarbeitung zwingend notwendig wird. Je nach Aufgabenstellung werden in einer 
Datenbank bestimmte Objekte behandelt, z.B. chemische Stoffe in einer Chemikaliendatenbank, Recyclingtechnologien in einer Abfallwirtschaftsdatenbank, Meßwerte von Luftschadstoffen in einer Meßwertdatenbank, Personen in einer Personaldatenbank. Diese Objekte werden durch bestimmte Merkmale, wie die Einsatzgebiete von Recyclingtechnologien oder der Ort und die Zeit eines Meßwerts oder das Alter einer Person beschrieben. Ein Beispiel für ein Merkmal zur Charakterisierung des Umweltverhaltens eines chemischen Stoffes ist die akute Toxizität für Fische bzw. Daphnien (s. Abb. 1)

Dieses Beispiel ist dem Anmeldebogen entnommen, auf dem die Melder für neu in den Verkehr zu bringende Stoffe im Rahmen des Chemiekaliengesetzes ihre Angaben zu vorgegebenen Merkmalen abgeben müssen. Das Beispiel soll weiter zeigen, daß ein Merkmal nicht nur aus einem einzelnen numerischen Wert oder einem einzelnen Text besteht, sondern selber aus komplexen Unterstrukturen bestehen kann, den sogenannten Untermerkmalen. 


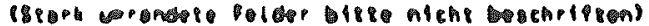

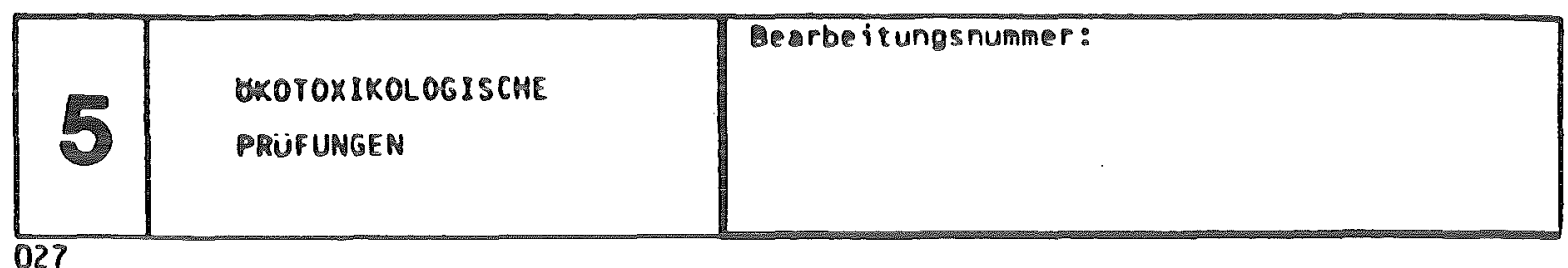

S.1 UIRKUNGEN AUF ORGANISMEN

5.9.1 Akure Toxizitat für Fische

$\mathrm{LC}_{0}:$ mg $11: \mathrm{LC}_{50}:$ $\mathrm{mg} / 1$

$\mathrm{LC}_{900^{\circ}}$ $\mathrm{mg} / 1$

Verwendete Art(en):

Testdaver:

คre thode:

Bemerkungen:

Für die Untersuchungen verantwortliche stelle: vertr.

5.1 .2 Akute Toxizität für Daphnien

$\mathrm{EC}_{0}:$ $m g / 1: E C_{50}:$ $m g / l$

EC $900^{\circ}$ $\mathrm{mg} / \mathrm{l}$

Tesidauer:

Methode:

Bemerkungen:

Für die Untersuchungen verantwortliche Stelle: verit.

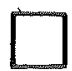


Die Datentypen der Untermerkmale sind in diesem Beispiel dimensionsbehaftete numerische Werte, normierte Begriffe und Texte. In Abb. 2 wird eine Angabe zu dem Merkmal aus Abb. 1 mit Testdaten an Hand eines INFUCHS-Wiedergabebildschirms gezeigt.

INFUCHS : Hiedergabe

00140

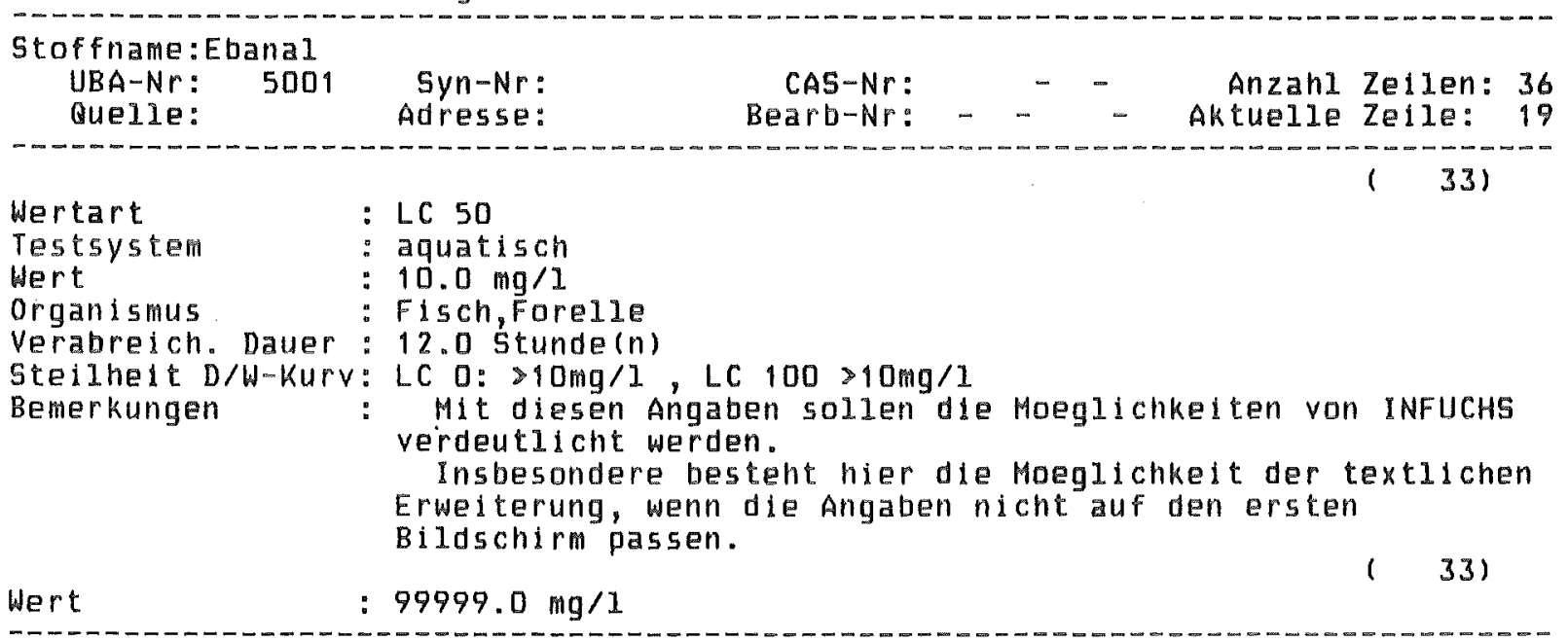

MELDUNGEN :

Welche Datentypen denkbar sind, zeigt die Abb. 3 an Hand eines Bildschirms zur Änderung von Angaben zum Demonstrationsmerkmal "Demo-Merkmal-Stoffbezug".

INFUCHS Aendern:Demo-Gruppe/Demo-Merkmal-Stof́fbezug

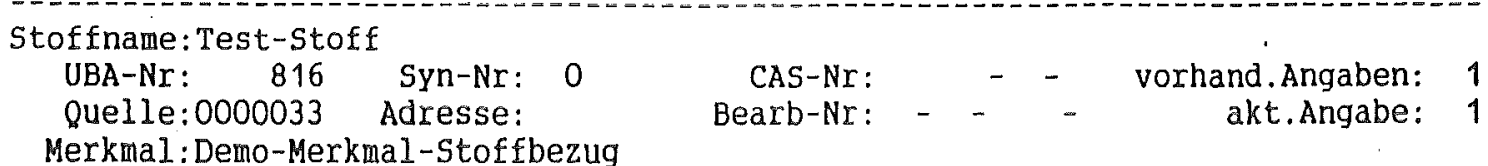

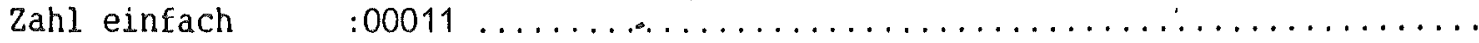

Zahl multipel :

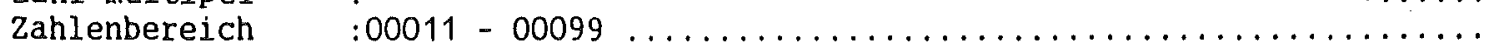

Zahl mit Einheit $:>\ldots \ldots \ldots \ldots \ldots \ldots \ldots$ Kelvin

Zahlenber.m.Einhei: $\ldots \quad 10.0 \ldots-\ldots 20.0 \ldots \mathrm{mg} / \mathrm{kg}$

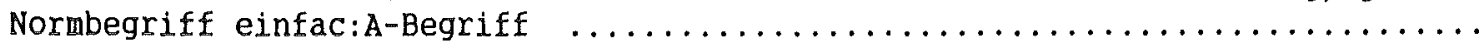
Normbegriff multip:A-Begriff B-Begriff C-Begriff D-Begriff E-Begriff $+\ldots \ldots$ Freitext $\quad$ Freitext-Zeile 1 mit Schlagwort 1 und Schlagwort2 + 
Merkmale zur Beschreibung des Umweltverhaltens eines chemischen Stoffes können aus beliebigen Kombinationen von Untermerkmalen und den damit verbundenen in $\mathrm{Abb}$. 3 aufgezeigten Datentypen zusammengesetzt sein.

Auf Grund des naturwissenschaftlich/technischen Fortschritts und den nicht absehbaren Anforderungen der Anwender an die Vielfalt der Merkmale und an deren Differenzierungen im Sinne von Unterstrukturen, ist eine endgültige Festlegung auf bestimmte Merkmale und Untermerkmale und damit auf fest definierte Datenstrukturen nicht möglich, wenn man vermeiden möchte, daß bei jedem neuen Merkmal bzw. neven Unterstrukturen sowohl die Datenstrukturen als auch das Programmsystem mit erheblichen Programmieraufwand geändert werden müssen.

Die Beschreibung der Merkmale und der dazugehörigen Unterstrukturen werden deshalb getrennt von den Datendateien in einer Tabelle abgelegt, aus der sich das System bei einem Zugriff auf eine bestimmmte Angabe zu einem Merkmal in der Datendatei die Beschreibung holt, um deren Inhalt zu interpretieren. Aus den Strukturen der Datendatei ist deren Inhalt nicht mehr zu entnehmen.

Durch die Ablage der Merkmalsdefinitionen in einer Tabelle ergibt sich der entscheidende Vorteil, daß auf Anforderung der Anwender neue Merkmale vom Datenbankverwalter innerhalb weniger Minuten eingerichtet werden können, ohne daß irgendwelche Programmierarbeiten notwendig sind. Für die Zuordnung zwischen der logischen Merkmalsbeschreibungen in der Tabelle und den physischen Datenstrukturen bei der Ablage der Angaben zu den Merkmalen in der Datendatei sorgt das System dann automatisch.

Einen Ausschnitt aus der Tabellenbeschreibung eines Merkmals zeigt die Abb. 4. 
Merkmalsmane

-deutsch : oekotox.Pruefungen, Akut

-englisch: Eco-Toxicological Studies, Acute

Kommentar :

Logische Dateinr.: 18

Log. Freltextdat.: 8

Identifik. -Typ: U (U/H/P/Q/V/A/L/Z)

Wertart : OTAKT

Startwertkennung:

LIstenausgabetyp:

MELDUNGEN :

MERKMALSDATEI: Auflisten der Untermerkmale CHEMULI 1

\begin{tabular}{|c|c|c|c|c|c|c|c|c|c|c|c|c|}
\hline \multirow[b]{2}{*}{$\mathrm{Nr}$. } & Merkmal: & .Prut & funger & Akut & & & & & & \multicolumn{2}{|c|}{ Nummer: } & \multirow{2}{*}{$\begin{array}{l}7011 \\
\text { NuI1 }\end{array}$} \\
\hline & Untermerkmalsname & Tyр & Form & $A u f b$ & $\operatorname{InV}$ & FN/ & $P E$ & Lng & TU & op & $E / A$ & \\
\hline 01 & Wertart & 001 & 003 & 000 & 0 & $A 1$ & 00 & 060 & & 0 & & 0 \\
\hline 02 & Testsystem & 001 & 003 & 000 & 0 & A3 & 00 & 060 & & 0 & & $F$ \\
\hline 03 & Wert & 041 & 006 & 000 & 0 & G1 & 00 & 043 & & 0 & MK8 & $\mathrm{F}$ \\
\hline 04 & Wirkung & 001 & 003 & 000 & 0 & A) & 00 & 060 & & 0 & & $\mathrm{~F}$ \\
\hline 05 & Orqan 15 mus & 003 & 003 & 000 & 0 & A5 & 00 & 060 & & 0 & & $\mathrm{~F}$ \\
\hline 06 & Appl 1 kationsart & 001 & 003 & 000 & 0 & $A 4$ & 00 & 060 & & 0 & & $\mathrm{~F}$ \\
\hline 07 & Verabrelch. Dauer & 010 & 006 & 000 & 0 & 64 & 00 & 027 & & 0 & ZT2 & $\mathrm{F}$ \\
\hline 08 & Verabrel chung & 001 & 003 & 003 & 0 & $\mathrm{FT}$ & 01 & 060 & VA & 0 & & $F$ \\
\hline 09 & Steilheit $D / W$-Kurve & 003 & 003 & 000 & 0 & A6 & 00 & 060 & & 0 & & $\mathrm{~F}$ \\
\hline 10 & Versuchbeschr. & 001 & 003 & 003 & 0 & FT & 02 & 060 & VB & 0 & & $\mathrm{~F}$ \\
\hline 11 & Tes tme thode & 003 & 003 & 000 & 0 & $\hat{A} 2$ & 00 & 060 & & 0 & & $F$ \\
\hline 12 & Bemerkungen & 001 & 003 & 003 & 0 & $\mathrm{FT}$ & 03 & 060 & B & 0 & & $F$ \\
\hline 13 & Verantw. Stelle & 003 & 003 & 000 & 0 & $A B$ & 00 & 060 & & 0 & & $F$ \\
\hline 14 & Bewertungsstelle & 001 & 003 & 003 & 0 & FT & 04 & 060 & 5 & 0 & & $F$ \\
\hline
\end{tabular}

Gewuenschte Untermerkmalsnummer ? : 0 
Ein erhebliches Problem bei naturwissenschaftlich/technisch orientierten Datenbanken stellt die Verarbeitung von mit physikalischen Einheiten behafteten numerischen Werten dar. Nicht nur die enormen Dimensions-Unterschiede in Größenordnungen von über zwanzig Zehnerpotenzen, sondern insbesondere die normierte Speicherung der Einheiten stellen erhebliche Probleme dar.

Eine alleinige Speicherung des Zahlenwertes ohne Berücksichtigung der unterschiedlichen Einheiten (z.B. Angaben in $\mathrm{mg} / \mathrm{l}$ oder $\mathrm{g} / \mathrm{l}$ ) würde eine spätere Suche nach numerischen Werten unmöglich machen. Aus diesem Grund muß bei der Datenerfassung eine interne Norminierung der Zahlenangaben erfolgen. Die Norminierung erfolgt über entsprechende Tabellen, in denen Umrechnungsfaktoren zwischen den unterschiedlichen Dimensionseinheiten abgelegt sind. Um bei der Wiedergabe der Angaben die ursprüngliche Dimensionseinheit wiederherstellen zu können, wird diese Dimensionseinheit beim Wert mitgespeichert

Zusätzlich zu den komplexen Strukturen, den unterschiedlichen Dimensionseinheiten kommt in vielen Fällen die große Anzahl von Merkmalen, die das Objekt der Datenbank beschreiben sollen.

Ein Stoff in der Stoffdatenbank (INFUCHS) wird z.B. durch weit über hundert Merkmale mit den unterschiedlichsten Kombinationen von Untermerkmalen beschrieben Da ein Anwender normalerweise nicht alle in der Tabelle definierten Merkmale für seine Benutzersicht der Datenbank benötigt, wird die Beschreibung der Sicht eines Benutzers unabhängig vom Programm ebenfalls in einer Tabelle abgelegt. Auf diese Weise ist es möglich, für jeden Benutzer eine eigene Datenbank entsprechend seinen Anforderungen zu definieren. Die Benutzersicht ergibt sich durch die Zuordnung der Merkmale aus der Merkmalstabelle. Die Strukturen der einzelnen Merkmale müssen vorher in der Merkmalstabelle definiert werden (z.B wie in Abb. 4). Die Benutzersicht auf ein Merkmal kann darüberhinaus problembezogen pro Benutzer eingerichtet werden. In Abb. 5 und 6 sind zum gleichen Merkmal "Ökotoxikologische Prüfungen, akut" zwei unterschiedliche Benutzersichten am Beispiel von INFUCHS-Datenänderungsmasken aufgezeigt. Die Angaben in der Datendatei sind bei beiden Sichten identisch, nur erhalten beide Benutzer eine in Anzahl, Reihenfolge und Auswahl unterschiedliche Zusammenstellung von Untermerkmalen. Das ist notwendig, um z.B. Angaben zu einem Merkmal sowohl aus der Literatur, aus der nur zu bestimmten Untermerkmalen Angaben erhältlich sind, als auch aus dem Vollzug einer gesetzlichen Regelung faktenmäßig aufbereiten und dem gleichen Merkmal zuordnen zu können (vergleiche Abb. 5 und 6).

Die beschriebenen Datenbank- und Merkmalssichten sind pro Benutzer für die Bereiche Suchen, Wiedergabe und Pflege unterschiedlich definierbar. Ein Benutzer kann z.B. nur ein Merkmal pflegen, aber 50 Merkmale für die Wiedergabesicht benutzen wollen. 
Stoffname: Ebanal

UBA-Nr: $5001 \quad 5 y n-N r: 0$

Que11e:0000033 Adresse:

Merkmal: Oekotox. Pruefungen, Akut

$$
\text { Wertart }
$$

Testsysterif

Wert

: LC 50

:aquatisch

Wirkung

$: 2$

$:$

:F i $5 \mathrm{ch}$

Applikationsart

Verabredch. Dauer

Verabrejchung:

Steilheit D/W-Kurv:LC

Versuchoes chr

Tes the thode

Bemerkungen

Verantw. Stelle

Bewertungs telle

MELDUNGEN:

(1)

:OECD
CAS-Nr:

Bearb-Nr:

vorhand.Angaben: 5 akt.Angabe: 4

$10.0 \ldots-\ldots$

... 角 $/ 1$

$12.0 \ldots \ldots \ldots \ldots \ldots \ldots$ stunde $(n)$

LC 0: $>10 m g / 1$, LC $100>10 m g / 1$ gesetzte Dasen

: Mlt diesen Angaben solien die Moeglichkeiten von INFUCHS

: Labor Kunterbunt

\section{Stof fname: Ebanal}

UBA-Nr: 5001

Quelle:0000033

Syn-Nr: 0

CAS-Nr:

Bearb-

Merkmal:0ekotox.Pruef., Akut (einfach)

$\begin{array}{ll}\text { Wertart } & : L C 50 \\ \text { Wert } & :>\text {. }\end{array}$

Verwendete Art(en): Fisch

Testdauer

He thode

Bemerkungen

verantw. Stelle

OECं
$10.0 \ldots-\ldots \ldots m g / 1$

$12.0 \ldots \ldots \ldots \ldots \ldots \ldots \ldots \ldots \ldots$ stunde $(n)$

: Mit diesen Angaben solien die Moeglichkeiten von INFUCHS :Labor Kunterbunt

MELDUNGEN: 
Sind für einen Benutzer eine größere Anzahl von Merkmalen definiert, so können die Merkmale zur besseren Übersichtlichkeit in Gruppen zusammengefaßt werden. In Abb. 7 wird eine

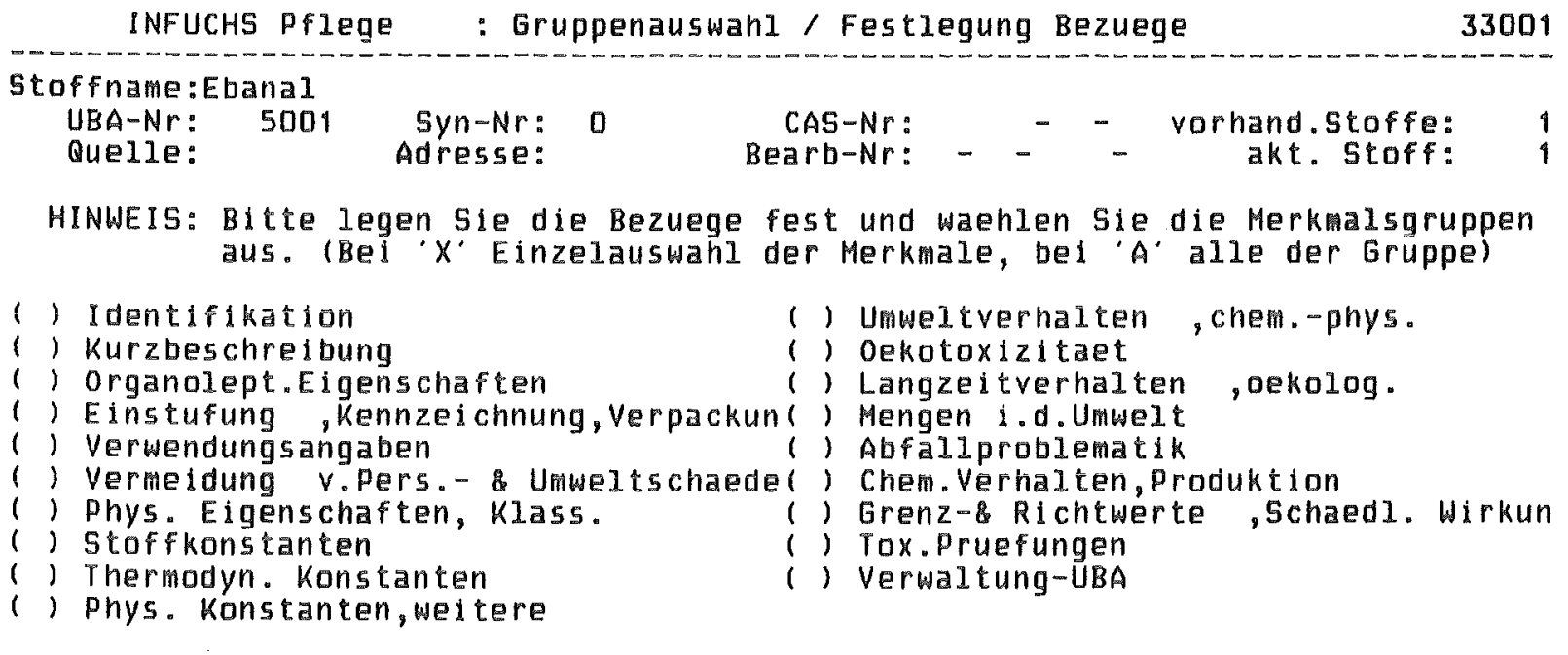

MELDUNGEN :

Benutzersicht, die in der Benutzertabelle abgelegt ist, bestehend aus 19 Gruppen gezeigt und zu einer Gruppe die dazugehörigen Merkmale (Abb. 8).

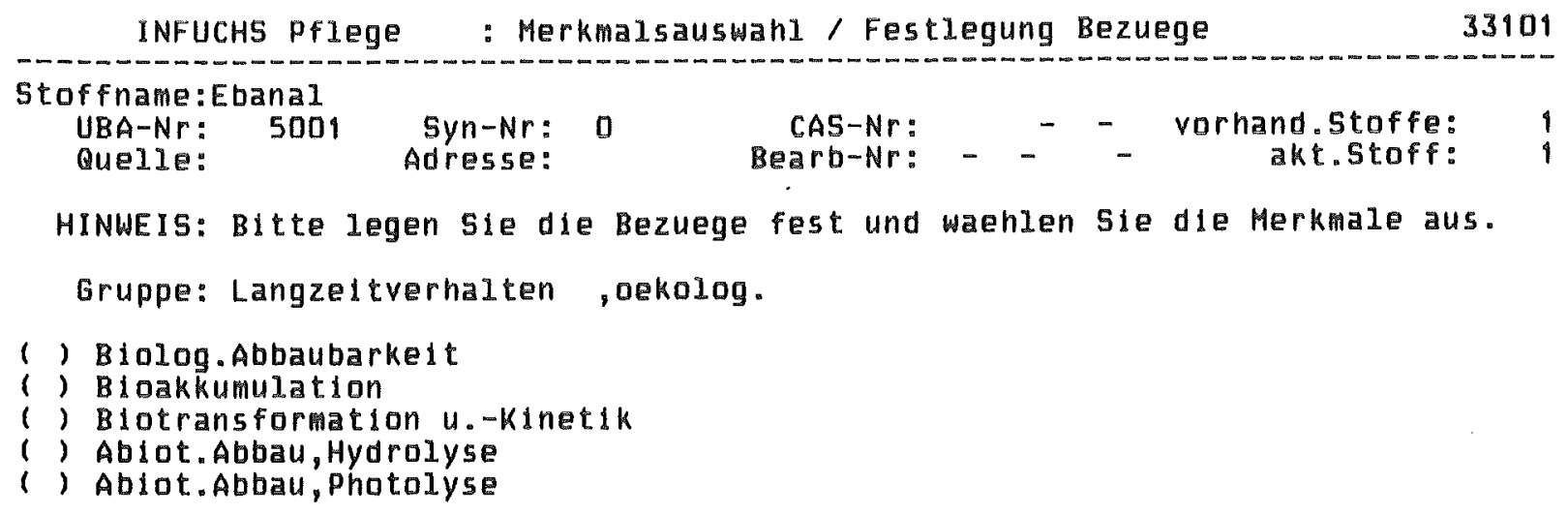

\section{MELDUNGEN :}

Bei der möglichen Vielzahl von Merkmalen, der Komplexität der Merkmalsstrukturen und der Menge von Angaben zu einem Merkmal, z.B. zum Merkmal "Ökotoxikologische Prüfungen, akut" kann es eine unvorhersehbare große Zahl von Tests (unterschiedliche Versuchstiere, 
Versuchsbedingungen, u.ä.) geben, ist es für einen Benutzer unmöglich, die Datenbank mit einer Kommandosprache, die die gleiche Vielfalt an Befehlen und Schlüsselwörtern bieten müßte, zu bedienen. Daher ist eine maskengeführte (z. B. mit dem Maskengenerator BIMAGE /BIM/) Dialogform unumgänglich. Die möglichen Nachteile der maskengesteuerten Dialogführung, wie z.B. die Starrheit des Dialogs, werden durch die zusätzliche Möglichkeit der Eingabe von Dialoganweisungen auf jeder Maske aufgelöst. So kann der Benutzer abweichend vom Normalablauf den Dialog knapper oder ausführlicher gestalten. Darüberhinaus kann der Benutzer über sog. Benutzeroptionen den Dialogablauf, das Wiedergabe-Layout oder auch die Sprache für alle Masken, Texte und Meldungen auswählen. Über alle Möglichkeiten kann sich der. Benutzer auf Hilfe-Masken jederzeit durch die Eingabe eines Fragezeichens informieren. Ein Beispiel für eine Hilfe-Maske wird in Abb. 9 gezeigt.

INFUCHS : Erlaeuterung des allgemeinen Steuerfeldes

Hit dem allgemeinen steuerfeld kann 1 fin INFUCHS der Normalablauf des

Dialogs wie folgt beeinflusst werden:

\begin{tabular}{|c|c|}
\hline $\begin{array}{l}\text { " } \\
\text { H } \\
\text { H }\end{array}$ & $\begin{array}{l}\text { Ende der Dialogsitzung } \\
\text { Abbruch und Rueckkehr auf die darueberldegende Ebene } \\
\text { Weiter, d.h. Veriassen elner Bildschirmwiederholungsfolge } \\
\text { Ausdruck der Bildschirmmaske auf standardsitzungsprotokold } \\
\text { Erlaeuterung des betreffenden Feldes }\end{array}$ \\
\hline $\mathrm{rad}$ & 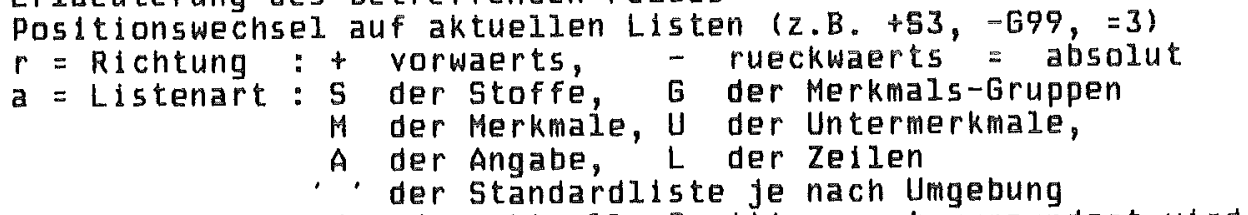 \\
\hline$+t$ & $\begin{array}{l}\text { Positionswechsel auf das Ende der standardilste } \\
\text { Positionswechsel auf den Anfang der standardilste }\end{array}$ \\
\hline
\end{tabular}

MELDUNGEN :

Die Suche nach Objekten in einer Datenbank (z.B. Stoffen in INFUCHS) über Ausprägungen zu Merkmalen bzw. Untermerkmalen in einer komplexen Umgebung muß durch geeignete Maßnahmen unterstützt werden. Ein Hauptproblem stellt die Suche nach Zahlenwerten mit Dimensionseinheiten dar. Sucht der Benutzer z.B. alle Stoffe mit einer akuten Fischtoxizität von 20 bis $50 \mathrm{mg} / \mathrm{l}$, so muß das System eine Umrechnung in die intern gespeicherte nomierte Darstellungsform (normalerweise in SI-Einheiten) vornehmen. Das erfolgt über die bereits erwähnte Umrechnungstabelle, ohne daß der Benutzer selbst eine Umrechnung vornehmen muß oder eine Suche über Werte überhaupt unmöglich wäre. Gehört der gesuchte Wert zu einem Untermerkmal, muß das System bei kombinierten Suchen mit anderen Untermerkmalen den logischen Zusammenhang auf der Ebene der Angaben zu dem Merkmal sicherstellen.

Bei der Suche nach nomierten Begriffen oder Schlagworten aus Texten muß das System dem Benutzer die Begriffs- bzw. Schlagwortlisten zur Auswahl vorblättern können. 
Um wiederum keinen Programmieraufwand bei der Einrichtung von Merkmalen in der Datenbank zu erzeugen, erfolgt die Unterstützung der Suche dynamisch aus der Merkmalstabelle.

\section{Ausblick}

Hauptziel beim Aufbau eines Datenbanksystems muß es sein, weder feste Daten- noch Programmstrukturen zu erzeugen. Eine Möglichkeit ist die beschriebene Verlagerung in unabhängige Tabellen, oft auch unter dem Begriff Data Dictionary zu finden, über die dann die dynamische Steuerung des Systems erfolgt. Trotzdem wird man damit nicht alle Probleme gelöst haben, denn der naturwissenschaftlich/technische Fortschritt wird auch zukünftig bisher nicht vorhersehbare Anforderungen erzeugen.

Aber die Umsetzung dieser Anforderungen ist im Rahmen des hier beschriebenen Ansatzes vermutlich wesentlich einfacher, da sich ein großer Teil durch Erweiterung bestehender bzw. durch Einrichtung zusätzlicher Tabellen abfangen lassen wird. 


\section{Literturverzeichnis}

/BIM/ BIMAGE

Bildschirmmaskengenerator der Fa. GSDmbH, Berlin

/MIE 85/ Mie, F.

Zur Terminologie und Typologie von Fakteninformationssystemen

Nachrichten für Dokumentation 36, 1985, Nr. 2, 5. 66 ff.

/PAG 83/ Page, B., Stopp, M.

Der Einsatz des Programmentwicklungssystem NATURAL bei der Realisierung von

Umweltdatenbanken

In: Der Senator für Inneres Berlin (Hrsg.), 20. Erfahrungsaustausch

ADV-Bund/Länder/Kommunaler Bereich, Münster, März 1983, S. 167-173

/STO 83/ Stopp, M.

INFUCHS - Die Faktendatenbank für Informationen über chemische Stoffe

In: Deutsche Gesellschaft fur Dokumentation, DGB-Schrift (OLBG-3), 2/83, 5. 233-239

/STO 86/ Musgrave, A.J., Page, B., Stopp, M.

INFUCHS - Ein Informationssystem für Umweltchemikalien, Chemieanlagen und

Störfälle

In: Page, B., Informatik im Umweltschutz, Anwendungen und Perspektiven,

Oldenburg Verlag, München 1986

/UMP 85/ Umweltbundesamt

Was ist UMPLIS?

Berlin, 1985 

DV-gestützte Umweltplanung auf der Basis von Geländedaten

W. Gillessen

IABG Ottobrunn

\section{Zusammenfassung:}

Methodik und Möglichkeiten der Rasterdatenverarbeitung - speziell der digitalen äquidistant gerasterten Gelände-/Umweltdaten werden vorgestellt.

Die in der IABG vorhandenen Datenbestände und Softwaremodule werden mit Beispielen aus der Umweltplanung beschrieben.

Die Visualisierung der Ausgangsdaten sowie der Planung an Farbmonitoren, wird als unabdingbares, zukïnftiges Arbeitsmittel erläutert.

Abschließend wird die Einbeziehung der Vektormöglichkeiten behandelt.

\section{Gliederung:}

1. Einführung

2. Rasterdatenverarbeitung

3. Daten und Software zur Umweltplanung

4. Anwendungen Umweltplanung

4.1 Farbdarstellung, Visualisierung

4.2 Raster-/Vektorverarbeitung

5. Ausblick 
1. Einführung

Seit mehr als 10 Jahren werden bei der IABG digitale Geländedatenbestände aufgebaut, gepflegt sowie Auswerte-Software und Darstellungsmöglichkeiten erkundet. Bis 1985 wurden mit diesen Geländeinstrumentarien ausschließlich Serviceleistungen gegenüber öffentlichen wie auch privaten Auftragnehmern erbracht. Seit 1985 werden zusätzlich zu diesen Dienstleistungen auch Daten sowie Software an interessierende Behörden oder Unternehmen abgegeben, damit diese damit eigene Planungen durchführen können.

Zur zeit wird der seit 10 Jahren entwickelte Rasterdatenansatz um Vektormöglichkeiten ergänzt; der geographische Bezug bleibt erhalten.

\section{Rasterdatenverarbeitung}

Einleitend sei aus Siemens "SICAD" zitiert, um einen Eindruck zu geben:

Digitale Rasterverarbeitungstechniken finden in der Umweltplanung anstelle oder in Ergänzung zur Vektortechnik immer mehr Anwendungen. Hauptvorteile sind die schnelle Datenerfassung mit Scannern, die schnelle Datenausgabe mit Rasterplottern, die einfache Datenstrukturierung in Datenbanken und die einfacheren und kürzeren Programme für die Datenverarbeitung. Zudem lassen sich spezielle Abbildungen, wie zum Beispiel Satellitenbilder oder topographische Karten, in der Vektortechnik nicht darstellen.

Topographische Karten, Thematische Karten und Satellitenbilder sind aber gerade die wesentlichen Grundlagen für raumbezogene Planungen. Sichere Analysen und Prognosen sind nur unter Einbeziehung aller genannten Informationsquellen möglich. Dies verlangt nach einem leistungsfähigen Werkzeug für die Verarbeitung flächenhafter Strukturen, die klassifiziert und interpretiert werden müssen und deren konkurrierendes Verhalten in Verschneidungs-, überlagerungs- oder Ausbreitungsmodellen $\mathrm{zu}$ analysieren ist. Die ständige Pflege und rasche, problemlose Fortführung des Datenbestandes muß gewährleitet sein.

Das IABG-Softwaresystem ist ein Rasterdaten-Verarbeitungssystem der oberen Leistungsklasse, das alle Instrumente für die Eingabe, Speicherung, Verarbeitung von Rasterdaten anbietet. Es erfüllt mit seinen praxiserprobten Komponenten alle Anforderungen aus den Umwelt-/Planungs- und kartographienahen Anwendungsbereichen. 
Die IABG entschied sich 1976 konsequent die Rasterdatenverarbeitung aufzubauen, aus folgenden Gründen:

- Hauptspeicher + Plattenspeicher werden drastisch preiswerter

- Datenerfassung vom Flugzeug, Satellit sind rasterorientiert

- die Ausgabemedien sind rasterorientiert Rastermonitore, elektrostatische plotter u.ä.

- die Software zur Verschneidung und Austwertung wie auch zum Datenhandling ist einfacher und leichter wartbar

- viele Umweltfragestellungen lassen sich nur mit zu hilfenahme von Höhendaten beantworten: Erosion, visuelle Beeinträchtigung, Schadstoffausbreitungen, ... Höhendaten auf Vektorbasis organisieren mit einer Genauigkeit von z.B. $50 \mathrm{~m}$ in $x y$ ist sehr kompliziert. Dagegen 200 Millionen Matrixelemente der Bundesrepublik Deutschland handeln ist mit heutigen Rechnern keine Schwierigkeit.

Die DV-Entwicklung zu Rechnern mit virtuellem Betriebssystem und großem Hauptspeicher bestätigt unsere damalige Entscheidung. 
Der abzubildende Merkmalsraum beschränkt sich nicht allein auf die Erdoberfläche (Höhe und F1ächennutzung) sondern auch auf Zustände in und über der Erdoberfläche. Nachfolgendes Bild 1 erläutert dieses:

$W e t e x$
UKW-VExSOI9Uחg

Fernsen-

vexsorgung

Grurdwasser

Ergiebigkeit.

Qua IIt at

Vexsorgungs-

Ieitungen

Gas. Wasser. EIektrizitbto $I \in I \in E O R$ 
Dabei wird der jeweilige zustand in seinem örtlichen (UTM, Gauß-Krüger, - oder geographischen Koordinaten) Bezug sowie mit seiner zeitzugehörigkeit festgehalten und im Rechner hinterlegt.

Abbildung des Raumes/der Zeit auf Matrił/Quader; diskrete Zeitintervalle

$$
\pm: I R^{3} \times I R_{T} \rightarrow I I^{3} \times I I_{T}
$$

Einem Punkt auf der Exdoberfläche $(x, y,(z))$ wird eine Adresse (iX, iY) sowie ein Codewert zugewiesen; der gleiche Codewert ist für alle Punkte im Intervall

gültig.

$$
[i X * D X,(i X+1) * D X] \times[i Y * D Y,(i+1) * D Y]
$$

Am Beispiel der Generierung eines digitalen Höhenmodells (DHM) sei diese Abbildung nochmals erläutert:

Aus Höhenlinienkarten (orohydrographischen) in einem Koordinatensystem (GK, UTM) erfaßt man an Digitalisiertischen - oder über Scanner mit anschließender Wertezuweisung - alle Höhenlinien und auch die Bergspitzen sowie vorhandene Talpunkte (anderes Kartenmaterial hinzuziehen). Je nach Kartenmaßstab und Erfassungsgenauigkeit wählt man die Rasterung der Höhenmatrix.

Beispiel: $1: 50.000 \rightarrow-$ Rasterung $25.4 \mathrm{~m}$ oder $12.7 \mathrm{~m}$

Man trägt alle erfaßten Polygone und Einzelpunkte als Meßwerte in die Rastermatrix ein. Fallen mehrere Meßwerte in das gleiche Raster, so bildet man Mittelwerte oder wähIt das Maximum. Anschließend werden mit einem Rasterinterpolator den nicht besetzten Matrixelementen Werte zugewiesen. 
Da für uns die ca. 200 Millionen Höhendaten für die Bundesrepublik Deutschland immer noch eine wesentliche Datenbasis für lokale Planungen sind, hiernoch einige Bemerkungen dazu:

Bemerkung:

1) Bruchkanten und Gewässerinformation lassen sich berücksichtigen.

2) Zweckmäßigerweise wird zur Interpolation kartenblattschnittrei gearbeitet. Eintragung von vielen erfaßten Karten in die startmatrix.

3) Die Höhendaten können ebenso aus einer photogrammetrischen Auswertung kommen.

4) Die Rasterung sollte < mittlerem Kartenfehler +

$$
\text { Digitalisierfehler }
$$

gewählt werden; die Rechenzeit geht i.a. wenigstens quadratisch mit der Rasterweite ein.

Halbierung der Rasterung $\rightarrow-\rightarrow$ Vervierfachung der Rechenzeit;

5) Selbst wenn die Genauigkeit der Höhenwerte im Integerbereich liegt, sollte die Höhenmatrix H (2000, 2000) als RealzahI oder als skalierter Integer gewählt werden.

Beispiel: $12=12.0$ od e r $12 \rightarrow 12 * 8 \quad 0 . \ddot{a}$. IABG nutzt für diese Aufgaben den Vektorrechner siemens VP200 mit 56 MB nutzbarem Hauptspeicher und bis zu 533 MFLOPS. 
Rasterdatenverarbeitung

Realität

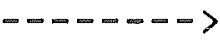

Abbildung diskrete Werte

(Matrix)

o d e $x$ :

jeder Raum-Zeit-Punkt $(x, y, z, t)$

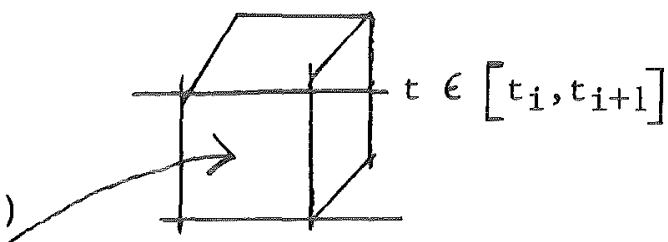

fällt in ein Quaderelement

$\mathrm{IR}^{3} \times \mathrm{T}_{\mathrm{IR}}$

Kontinuum

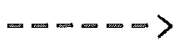

$----m$
II $^{3} \mathrm{X} \quad \mathrm{T}_{\mathrm{II}}$

Integerraum

Auf der Erdoberfläche wird ein rechtwinkliges Koordinatensystem gewählt (UTM, GK,...) und ein Gitternetz mit einer Maschenweite (= Rasterung) definiert.

Damit ist einer $(X, Y)$-ortskoordinate, die den Koordinatenursprung in der südwestecke hat, eine Gitterposition = Matrixelement zugeordnet.

$$
(X, Y) \rightarrow(I X, I Y), \quad \begin{aligned}
i X & =X / D X+1 \\
i Y & =Y / D Y+1
\end{aligned}
$$

Úblicherweise ist die Maschenweite $D X=D Y ;(I X, I Y)$ ist die Adresse. Liegt ein DHM vor, so findet man den für $(X, Y)$ gültigen Höhenwert $\mathrm{z}$

$$
\mathrm{z}=\operatorname{DHM}(I X, I Y)=\text { Wert (etwa } 485[\mathrm{~m}])
$$




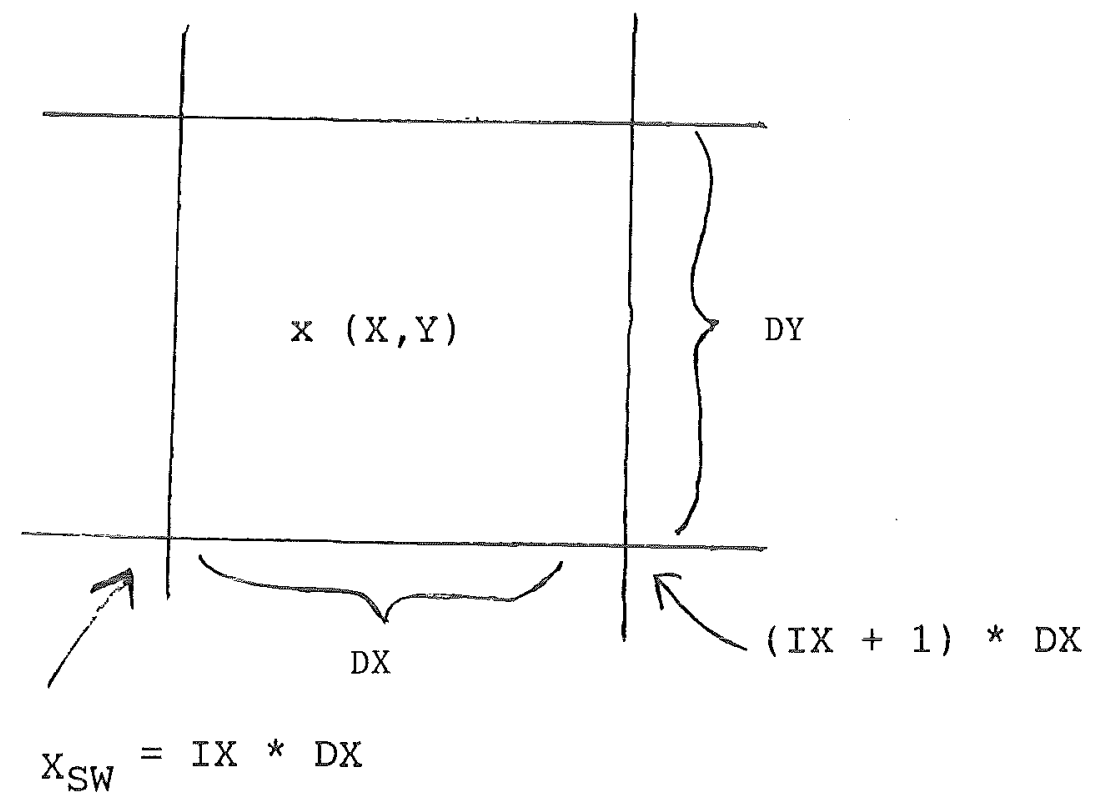

Allen $(X, Y)$ die in $\begin{aligned} & {[I X \times D X,(I X+1) * D X]} \\ & {[I Y \times D Y,(I Y+1) * D Y]}\end{aligned} \quad$ liegen, wird der gleiche Höhenwert zugeordnet.

Außerdem könnten noch

$$
\begin{aligned}
& \text { Code }_{F}=\text { Flächennutzung }(I X, I Y) \\
& \text { Code }_{W}=\text { Luftdruck }(I X, I Y) \text { zur zeit } t_{i} \text { am Boden } \\
& \cdot \\
& \text { Code }_{B}=\text { Bevölkerungsdichte }(I X, I Y), 1986
\end{aligned}
$$

vorliegen. 
Hat man einmal eine sW-Ecke als Ursprung definiert und die Rasterung vorgegeben, so braucht man nur noch

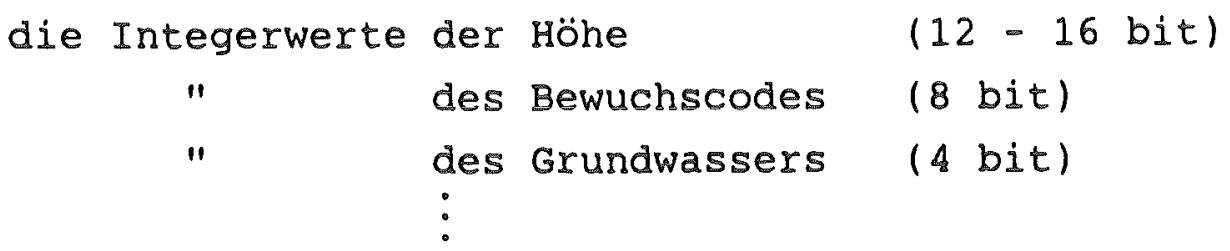

sequentiell abzulegen (zeilenweise von west nach Ost) und spart sich die Abspeicherung der UTM oder GK-Ortskoordinaten $z u$ jedem punkt.

Die Berührungsängste der Planer mit den modernen Datenverarbeitungsmöglichkeiten hatten bis etwa 1977 inre Berechtigung, da erst zu diesem Zeitpunkt der Durchbruch bei der Massendaten-Verarbeitung gelang (Rechner mit $\triangle M B$ Hauptspeicher, großen Platten, schnellem Bus, schnellem I/O, Preisklasse 0,5 Mio DM).

Die Erfolge der Datenverarbeitung gerade auf dem Gebiet thematischer Kartenerstellung und Planungsunterstütung mit digitalen Geländedaten sind heute nicht mehr zu ubersehen, obwohl immer noch preiswerte, qualitativ hochwertige Ausgabesysteme fehlen.

Gerade der universitäre Bereich mit seiner verantwortung für die Ausbildung des Nachwuchses sollte den Einstieg in die neuen Möglichkeiten der Datenverarbeitung nunmehr wagen, denn gerade jetzt ist beispielsweise auf den Gebieten softwareentwicklung und komfortablere Gestaltung der Mensch-Maschine-Kommunikation noch einiges $\mathrm{zu}$ leisten. 
3. Daten und Software zur Umweltplanung

Eolgende digitale Geländedaten liegen bei IABG vor. Die Datenbestände 2 bis 7 werden von IABG gepflegt. Die Daten aus 2.), 3 \mathrm{~b})$ c) d), 4 und 6 können gegen Lizenzgebühren abgegeben werden (siehe Bild 3 ). 


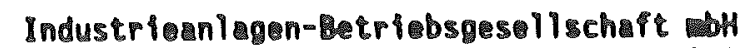

Abretiung To6. Tolefon 089-6008-3435

B i 1 d 3

- Gelindedatenverarbeltung -

Ubersicht uber die bei der IABS vorhandenen

digitalen Gelendedaten

Stand: September 1986

1. Datenbank TOPOGRAPHIE

Auflösung: 5" " 5" (Bogensekunden), BRD komplett umgerastert auf UTMKoordinatensystem in $101.6 \mathrm{~m} * 101.6 \mathrm{~m}$ und größere Raster.

Inhalt: Topographische Höhe sowie Bewuchs und Bebauung (in $10 \mathrm{Klassen}$ ).

2. Yon der IABG/TOG neu erfabte IABG-eigene Geländedaten aus Kartenmaterial Auflösung: $50.8 \mathrm{~m} * 50.8 \mathrm{~m}$, UTM-Koordinatensystem, GK

Inhalt: Topographische Höhe über NN.

bisher erfaßt: Baden-Wüttemberg, Rheinland-Pfalz, Luxemburg, Bayern incl. Alpenregion, ostfrankreich. BRD komplett in 1987.

3. Feingerasterte Gelände
a) LICHTENAU
Ausdehnung: $12.8 \mathrm{~km} * 12.8 \mathrm{~km}$
Auflösung: $6.25 \mathrm{~m} * 6.25 \mathrm{~m}$
b) GRAFENWÖHR
Inhalt : Höhe, Bewuchs, Bebauung
Ausdehnung: $22.0 \mathrm{~km} * 33.0 \mathrm{~km}$
Auflösung: $12.7 \mathrm{~m} 12.7 \mathrm{~m}$
Inhalt : Höhe, Bewuchs, Bebauung
c) KARWENDELGEBIRGE
Auflösung: $12.7 \mathrm{~m} * 12.7 \mathrm{~m}$
d) FICHTELGEBIRGE
Inhalt: Höhe
Auflösung : $12.7 \mathrm{~m} * 12.7 \mathrm{~m}$
Inhalt : Höhe

4. Klassifizierte und geosetrisch entzerpte LANDSAT-4 Daten

für Südwestdeutschland

Rasterung: $101.6 \mathrm{~m} 101.6 \mathrm{~m}$, UTM-Koordinatensystem.

5. Landschaftsdatenbank ( Yorbehaitsstrecken >

Aưlösung: $25 \mathrm{~m} \div 25 \mathrm{~m}$ (aus GK - Karten)

Vollständige Erfassung von Umweltdaten ( 16 thematische Dateien, 2.B. fur

Boden-, Flächennutzung, Klima, Wasserhaushalt, Biotope, etc. ; fur 10 Projektgebiete in den Kreisen Pinneberg-Stade, Cuxhaven-Wesermarsch, Mettmann-Essen-Bochum-Ennepe/Ruhr, Köln, Siegen-Hochsauer land-Marburg/

Biedenkopf-Waldeck/Frankenberg-Schwalm/Éder, Pirmasens-Südpialz, We instraße-Germersheim, Böblingen, Bodensee, Konstanz.

6. Strapen / Gewässer / Gesaindegranzen / Babaung / Devölkerungsdichten für Teile der Bundesrepublik Deutschland.

7. Hattkartierung < Hurster Kuste >

Inhalt: Boden, Bodennutzung, Fauna, Flora.

Höhendaten zwischen $-10 \mathrm{~m}$ und $+10 \mathrm{~m}$. 
Folgende Software zur Umweltplanung stellt IABG für 32 bit Rechner zur verfügung ( 5 iehe Bild 4,5 und 6 ):

B 11 id 4

\author{
Gelånde-/Unwelt-Sortware der I A G \\ Stand: September 1986
}

IABG-Gelände-Modul-System (IGM)

Module des IGM: (UNIX, "C")

- Schnittstelle zu Bildverarbeitungs-Ergebnisdateien und Scannern (Spot, Landsat, Hell, Photogrammetrie) = SBV

- Datenbankverwaltungssystem für Rasterdatejen: D B R (Auszüge, Handling, Überschreibung, Zupfen, Koordinatenübergänge (mit Tabellen))

- Displaysystem: D $\mathrm{S}$

Ausgabe der Basis und Ergebnisdatei

- Verschneidung, logische Veknüpfung von Rasterdateien $\left(v, 7, \sum_{i}\right.$ cixRasterdatei), "LOGIK", Ưberlagerung.

- Höhendaten-Software HSw, Relief, Hangneigung, Exposition (3D schnell, fein, optional), sicht

- Höhe + Flächennutzung: Farbrelief, Erosion

F R F

- Vektor-Datenerfassungs-/änderungs - Baustein, Attributzuweisung, Farbkontrolle, Füllroutinen

D E F

- Software-Biblothek: S B I B

a) Abstandsdateigenerierung für Punkt, Vektor, Fläche

b) Statistik

c) Versorgungsmode1le (Lärm-, Schadstoffe-, Strömungs-)

d) Geo-UTM-GK

- Menuesystem MEN UE. 
Die von der IABG bereltgestellte Gelende-Software orlaubt

- die Digitalisferung von Kartenvorlagen am elngebauten Digitaifslertisch mit Farbkontrollo fur die Attribut-Zuwelsung

- die Verarbeltung der gebrguchilichen Scanner-Formate (Heli, Spot, Optronics, Landsat)

- das Datenhandiling for groBe Rasterdatenbestande we Ausschnitt. Punkt. Zelle. Schlauch sowle das Zusammensetzen elnzelner Telldaten zu einem Gesamtdatenbestand

- die Verschneldung und logische Verknupfung von inhaltilich unterschledlichen Rasterdatelen:

mit diesem Auswertungs - und Oberlagerungsmodul können Funktionsund Rislkobewertungen durchgefohrt werden

- die Berechnung von Abstandsdatefen zu Punkten und Polygonzogen sowle das Furlen von geschlossenen Polygonzögen

- die Berechnung und Darstellung des Höhenrellefs (mit unterschiedifchem Sonnenstand), mit Hangneigung und Exposition

- die Berechnung des Farbrellefblides aus Höhen- und Figchennutzungsdaten.

Diese Basis-Software kann auf Wunsch organzt werden durch weltere spezielle Softwaremodule:

- Koordinatensystemtransformation (Gaubi-Kroger, UTh, goographisch)

- Mosalking (Zusammensetzen Ober Kartenblattgrenzen hinweg)

- Interpolation von groBen HöhenmeBwerte-Datelon

- BESTwAY und SUPPLY zur Berechnung von kUrzesten Pfaden und Versorgungsnetzen fOr "Glttersysteme (Straben, Kabol, Abuasser) 


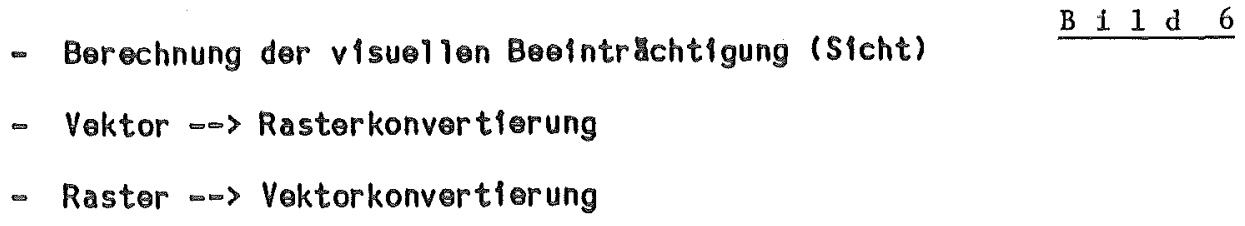

Die Software nutzt den vollon Hardwaro-Umiang eines dezentralen Arbeltsplatzes: den 32 bit-Rechner mit virtuellem Betriebssystem, den hochauflosenden Farbrastermonitor mit schneller Bllddarstellung von dor CPU sow 10 den Digltallsiortisch mit Farbkontrollmoglichkelt.

\section{Ein Instrumentarium zur Bewertung der Umweltrelevanz von geplanten Trassen}

Zunächst werden die vorhandenen Flächennutzungen (z.B. Landwirtschaft, Forstwirtschaf́t .. .) sowie die natürlichen Nutzungsgrundlagen im großräumigen Einwirkungsbereich der Trasse (und der Alternativirassen) erfaßt. Siehe umseitiges Bild: Beispieltrasse mit Einwirkungsbereich.

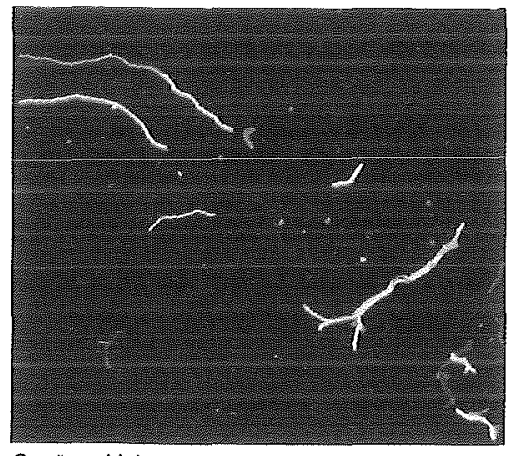

Gewässerblotope

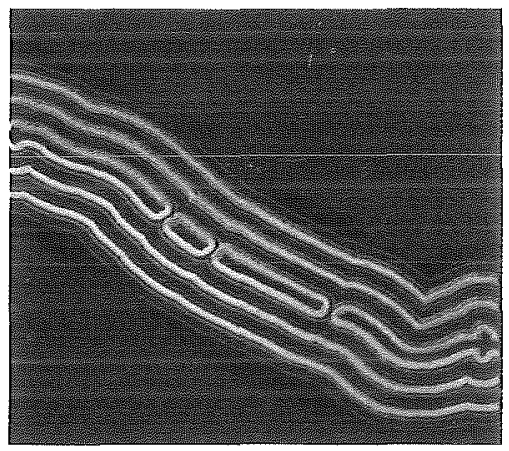

Abstandsdatel zur vorgegebenen Trasse mit Brücken

Im zweiten Arbeitsschritt werden die wesentlichen Umweltwirkungen, die mit dem Bau und dem Betrieb des Verkehrsträgers verbunden sind, wie Lärmund Schadstoffimmission, Zerschneidungseffekte sowle visuelle Beeinträchtigungen erfaßt. Über eine Datel ăquidistanter Abstandszonen (je nach Anwendung $20 \mathrm{~m}$ bis $100 \mathrm{~m}$ breit) kann die u. a. mit der Entfernung abnehmende Intensität der Umweltwirkungen berücksichtigt werden.

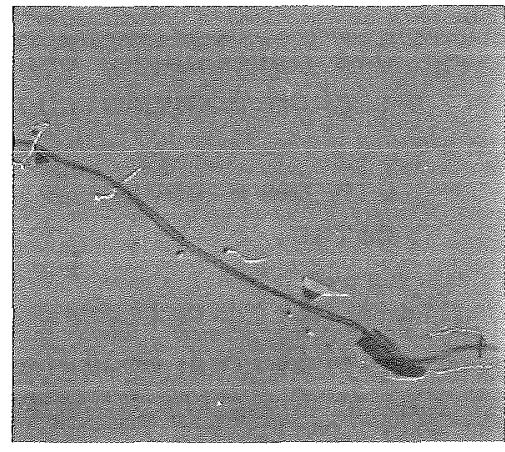

Beeintráchtigung der Gewasserblotope

Bewertet werden die direkt auf den Menschen und seine räumliche Umwelt einwirkenden Stobrefiekte. Das Beeintrăchtigungsrisiko für einzelne Funkilonen des Naturhaushaltes, wie z.B. für Arten- und Biotopschutz, wird in die Bewertung mit einbezogen.

Ein planerisches Konfllktmanagement wird erreicht über die răumliche Darstellung von Belastungs- bzw. Risikoschwerpunkten. Diese Darstellung geschieht wahlweise an einem Farbmonitor, als maßstabsgerechter Grauplot oder als Farbbild.

Änderungen in der technischen Ausführung, sowie alternative Trassenführungen lassen sich damit sofort in ihrer Wirkung auf die Umwelt simulieren. Die interaktive Arbeltsweise ist eine Stärke dieses modernen Planungsinstrumentariums.

Über die entsprechende ausgeweitete Untersuchung können zudem Entlastungseffekte im übrigen Netz (z.B.: bei Ortsdurchfahrten, die durch eine Umfahrung entlastet werden sollen) mitbilanziert werden. 
4. Farbdarstellungen, Visualisierung

Anwendungen, Umweltplanung

Die Darstellung der digitalen Geländedaten (Höhe und Fläcnennutzung) als Schummerungsbild bzw. Farbreliefbild ist Voraussetzung für alle Planungsmaßnahmen, die in einer Computersimulation an interaktiven Arbeitsplëtzen durchgespielt werden.

Das Umwelt/Planungsergebnis (die Jaumaßnahme) muß visualisiert werden mit der alternativen digitalen Landkarte im Hintergrund.

Wenn Karte und Auswertung auf dem gleichen Ausgangsdatenbestand aufbauen, wird die dargestellte Auswertung erst glaubhaft.

beispiele hierfür sind die im Diavortrag dargestellten Sichtdiagramme und simulierten faumaßnahmen. 
Das nachstehende Fotomaterlal wurde aus den IABG-Datenbeständen mit der eigenen Gelände- und B1ldverarbeltungssoftware an unserem S1emens APCCAD Monitor (32 bit Rechner, SINIX, Monitor $1280 \times 1024$ B11dpunkte) bzw. an unserem VTE-System ausgegeben und von dort abphotographiert.

Die Bllder 1 bis 6 stellen den Ausschnitt Schwarzwald - Rheingraben Ostfrankreich dar. Dabei wurden Höhenrasterdaten, Landsat-Mult1spektraldaten als Rasterdatel sowie die Euro-Datenbank des IFAG als Vektordatenbasis verarbeltet. Alle Daten wurden in das UTM 32U-Koordinatensystem (Bild 1-6: Siemens APC-CAD) transformiert.

B1ld 1: abgeleitetes Höhenreliefb1ld

B1ld 2: Landsat-Satellitendaten mit Bildverarbeitungsoptionen

B1ld 3: Höhenrelief mit Landsat komblnfert

B1ld 4: wie Bild 3 mit Vektordaten (IFAG), Höhenlinlen, Flußnetz und gefüllter Stadtinformation

Bild 5: Ausschnitt von Bild 4, Zoom, "Stuttgart"

B11d 6: 3-D oder Zentralperspekt1ve, gerechnet aus den Höhen-, Reliefund Landsat-Daten mit Höhenschnitten eingeblendet

Die Bilder 8 bis 12 behandeln Auswertungen mit einem feiner gerasterten (12 m Raster) digitalen Höhen- und Flächennutzungsdatenbestand und wurden an unserem VTE-System ausgegeben.

Bild 10: Farbreliefbild Lichtenau (alternative Landkarte)

Bild 11: Simulation der visuellen Beefnträchtigung durch Baumaßnahmen (Küh1turm $96 \mathrm{~m}$, Slcht aus Augenhöhe)

B1ld 12: 3-D-Bild, Simulation von Gebäuden im Gelände

Die hier zum Einsatz kommende IABG-Software war die UNIX, "C" und die "FTN"Software für den APC-CAD sowie die DEC-VAX-FTN-Software für das VTE-System. Alle wesentlichen Gelände- und Bildverarbeltungsroutinen sind inzwischen für den Siemens-Arbeltsplatz verfügbar einschließlich der neuen Rasterdatenbank-Software in "C". 
- 117 -
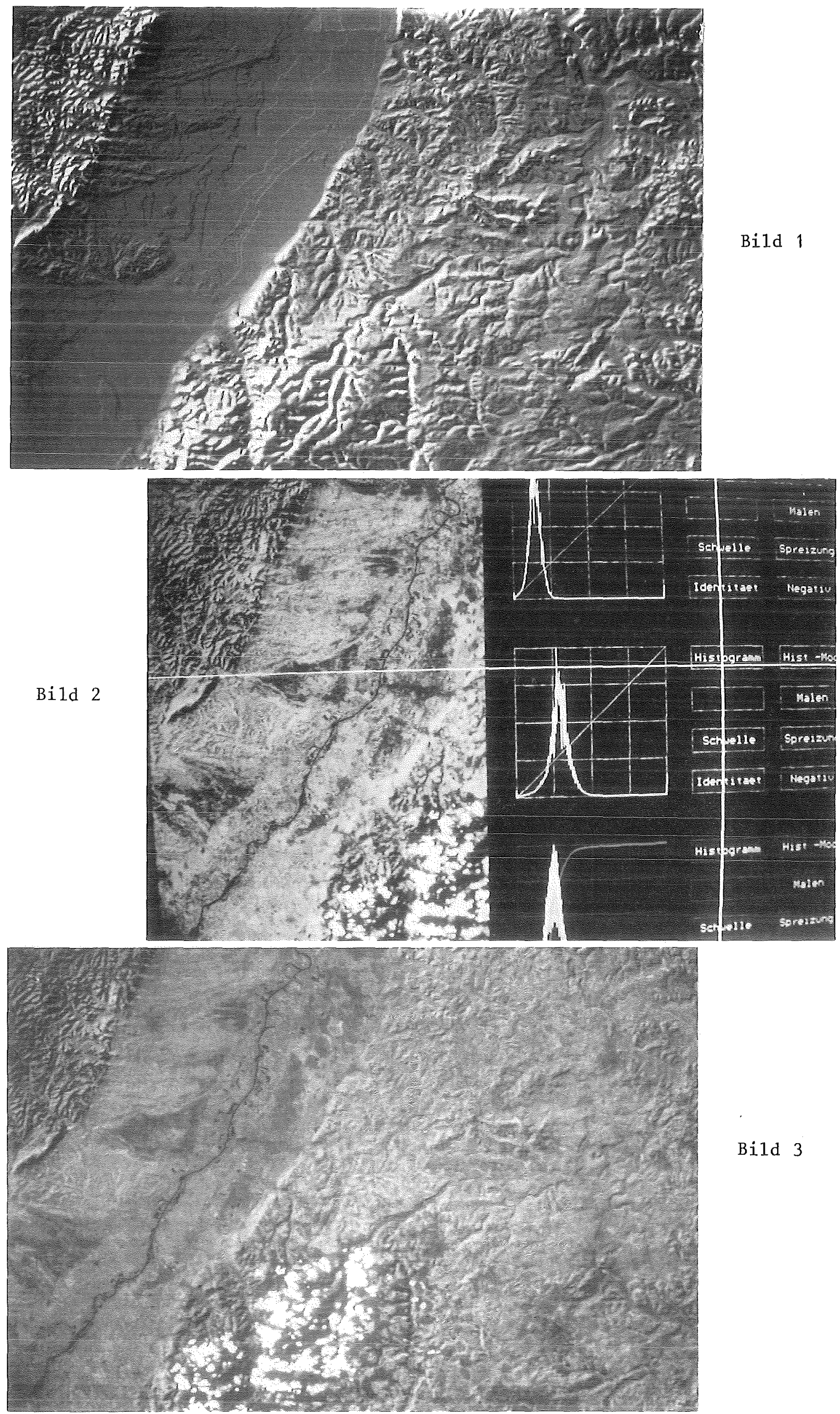

Bild 3 


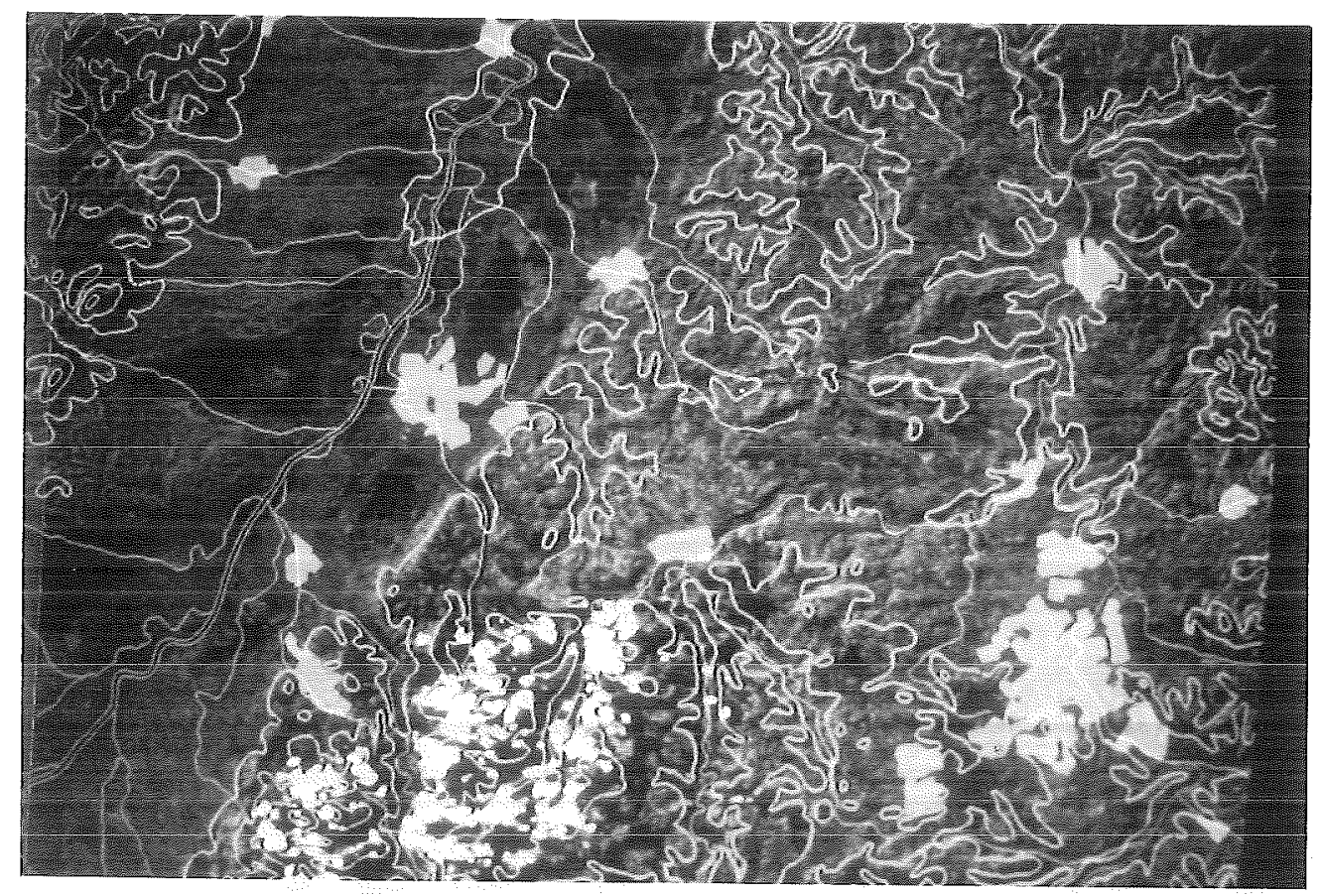

Bild 4
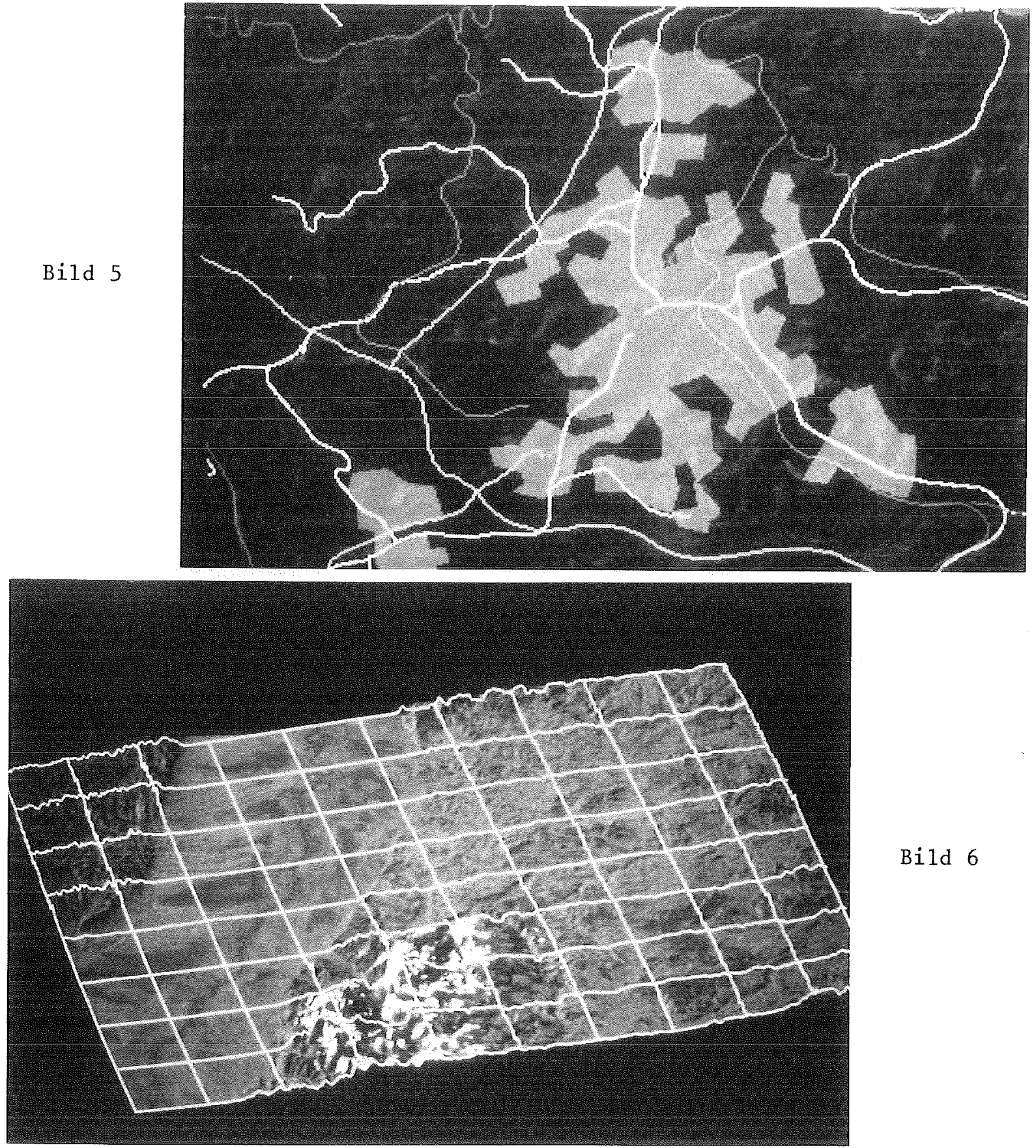

Bild 6 
B $\underline{1} \underline{1} \underline{\mathrm{d}}$

Risikobewertung der Weserquerung bei Bremen (Bundesverkehrswegeplanung

hier: ölkologische Bewertung)

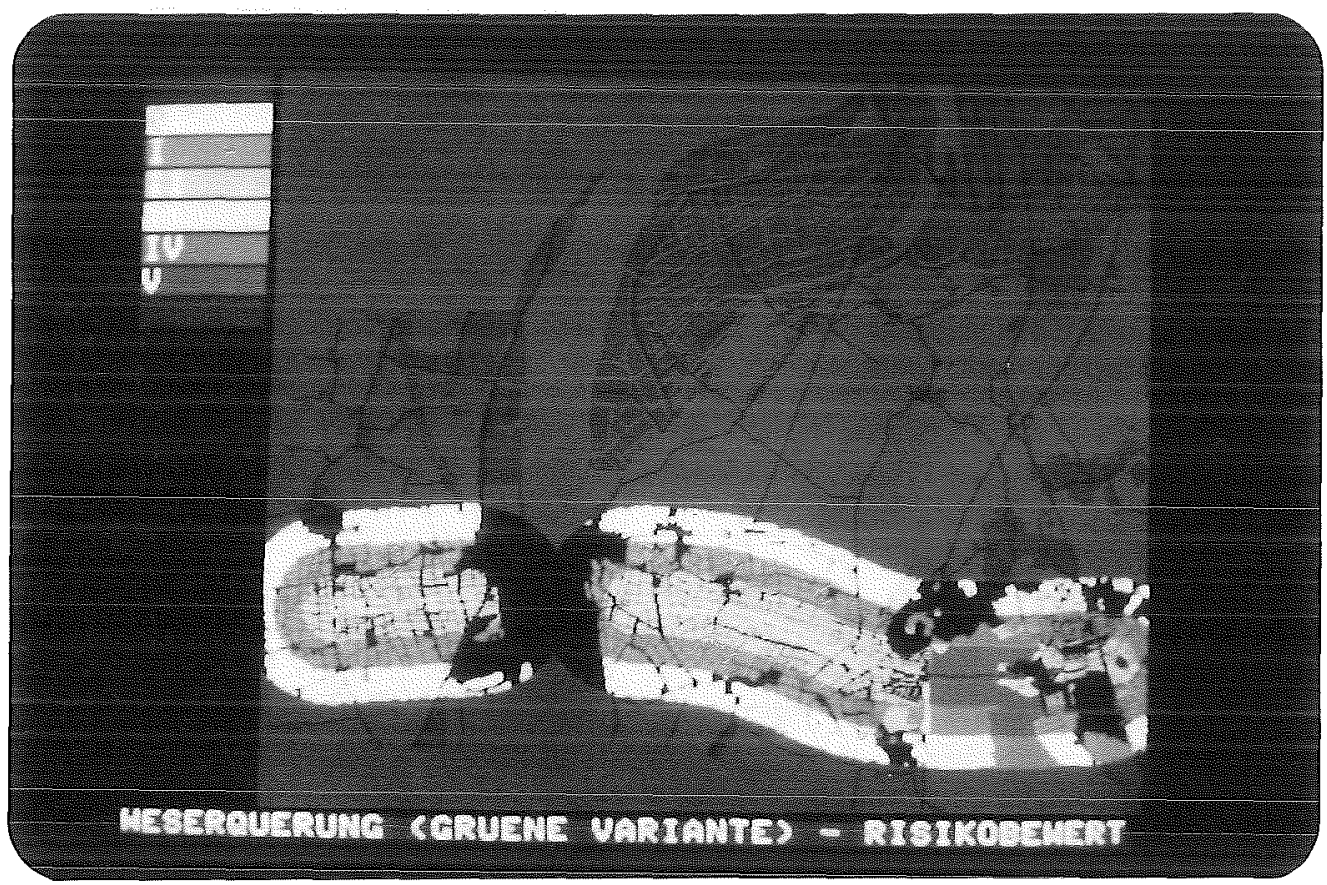




\begin{tabular}{|c|c|c|c|}
\hline $\begin{array}{l}\text { Ausbreitungs- } \\
\text { rechnungen. } \\
\text { Auf den IABG- } \\
\text { Rechnern liegen } \\
\text { verschiedene } \\
\text { Strömungs- und } \\
\text { Ausbreitungs- } \\
\text { modelle vor. } \\
\\
\text { Das auf unse- } \\
\text { rem Vektorrech- } \\
\text { ner SIEMENS } \\
\text { VP } 200 \text { instal. }\end{array}$ & $\begin{array}{l}\text { lierte mesoskali- } \\
\text { ge Sirömungs- } \\
\text { modell FITNAH } \\
\text { von Prof. Dr. } \\
\text { Wippermann, } \\
\text { TH Darmstadt, } \\
\text { nutzt dabei die } \\
\text { Geländeinforma- } \\
\text { tion (Höhe und } \\
\text { Bedeckung) zur } \\
\text { Berechnung der } \\
\text { Strömungsver- } \\
\text { hältnisse. Die }\end{array}$ & $\begin{array}{l}\text { daraus ableitba- } \\
\text { re Ausbreitung } \\
\text { kann anschlie- } \\
\text { Bend im 3D- } \\
\text { Gelände darge- } \\
\text { stellt werden, } \\
\text { um so die be- } \\
\text { einflußten Ge- } \\
\text { biete auszuwei- } \\
\text { sen. }\end{array}$ & $\begin{array}{l}\text { pringipiellen } \\
\text { Darstellungs- } \\
\text { möglichkeiten } \\
\text { für Partikelwol- } \\
\text { ken im Gelände. } \\
\text { Die oben ar- } \\
\text { wähnte aufwen- } \\
\text { dige Strömungs- } \\
\text { rechnung wurde } \\
\text { hierfür jedoch } \\
\text { noch nicht } \\
\text { durchgeführt. }\end{array}$ \\
\hline
\end{tabular}

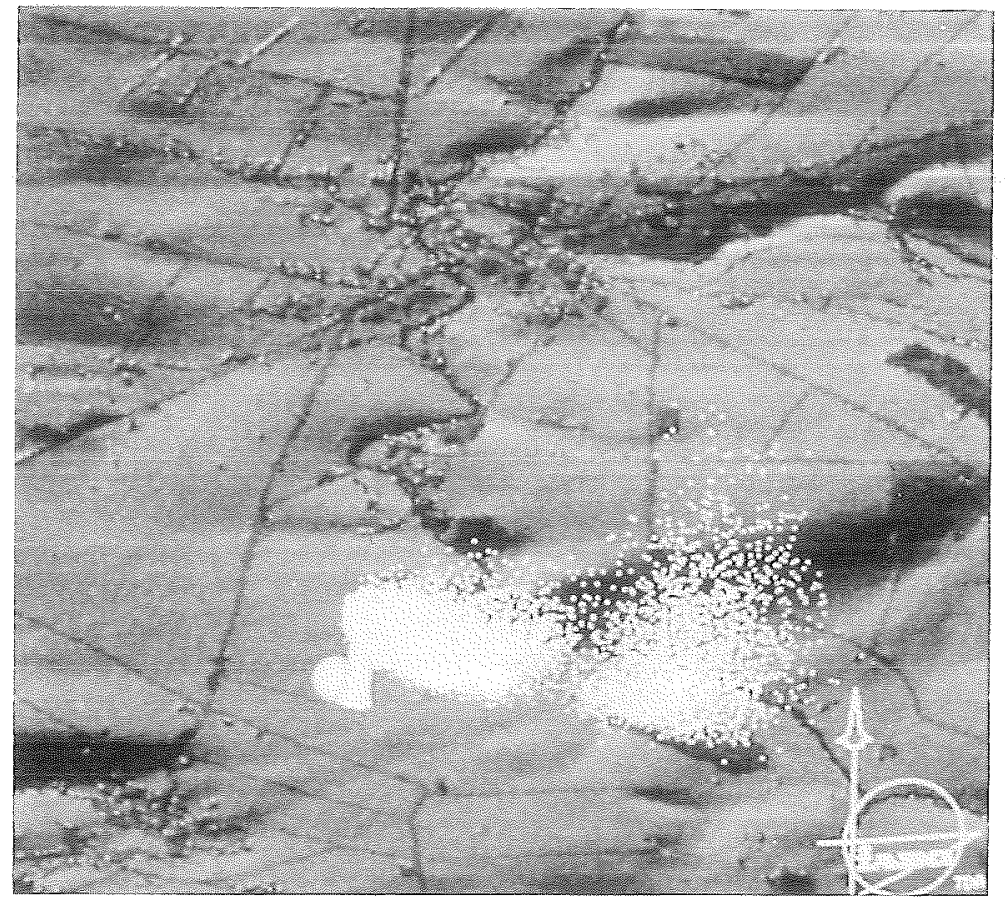

Visuelle Beeinträchtigung durch Trassierung - ähnlich wird der Ver1ängerungskorridor dargeste11t

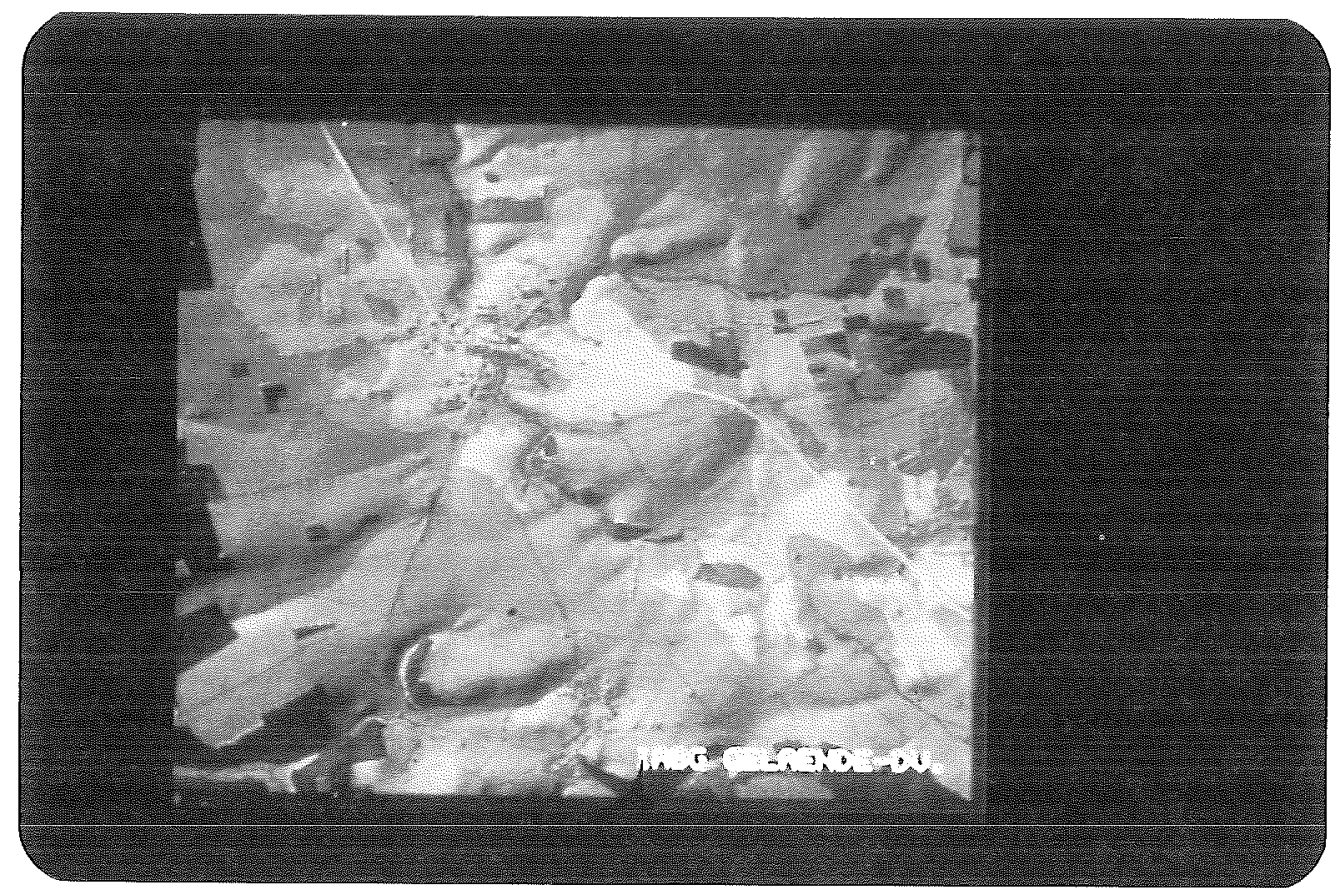




\begin{tabular}{|c|}
\hline $\begin{array}{r}\text { Farbreliefbild } \\
\text { abgebildet Lichte- } \\
\text { nau bei Paderborn } \\
\text { in einem Aus- } \\
\text { schnitt von } \\
6,4 \mathrm{~km} \times 6,4 \mathrm{~km} ; \\
\text { berechnet aus di- } \\
\text { gital vorllegenden } \\
\text { gerasterten Hö- } \\
\text { hen- und Flächen- } \\
\text { nutzungsdaten mit } \\
\text { einer Auflösung } \\
\text { von } 12,5 \text { m x } 12,5 \\
\text { m und dargestellt } \\
\text { an einem Farbra. } \\
\text { stermonitor mil } \\
512 \times 512 \\
\text { Bildpunkten. } \\
\text { Diese Darstel- } \\
\text { lungsform für digi- } \\
\text { tale Gelăndedaten } \\
\text { als Farbreliefbild } \\
\text { ist die zukünftige } \\
\text { lanungsunterlage } \\
\text { und wird das her- } \\
\text { kömmliche Arbei- } \\
\text { ten mit Karten } \\
\text { ergänzen oder } \\
\text { orsetgen. }\end{array}$ \\
\hline
\end{tabular}

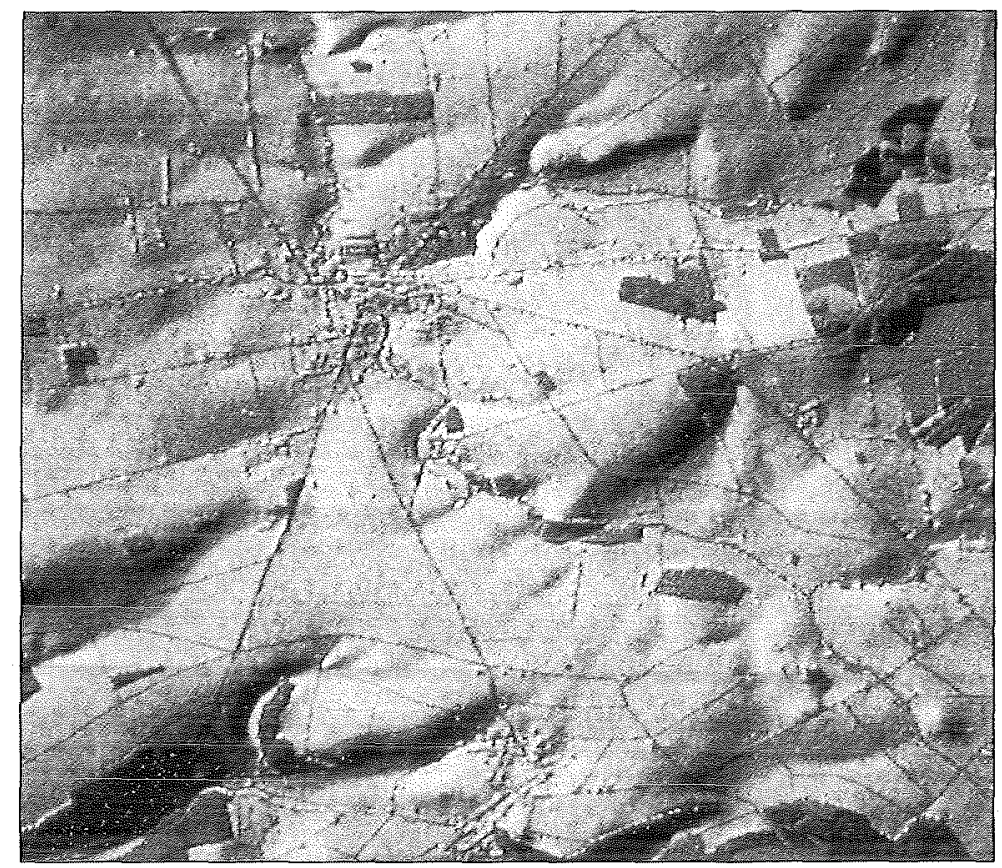

\section{Bild 11}
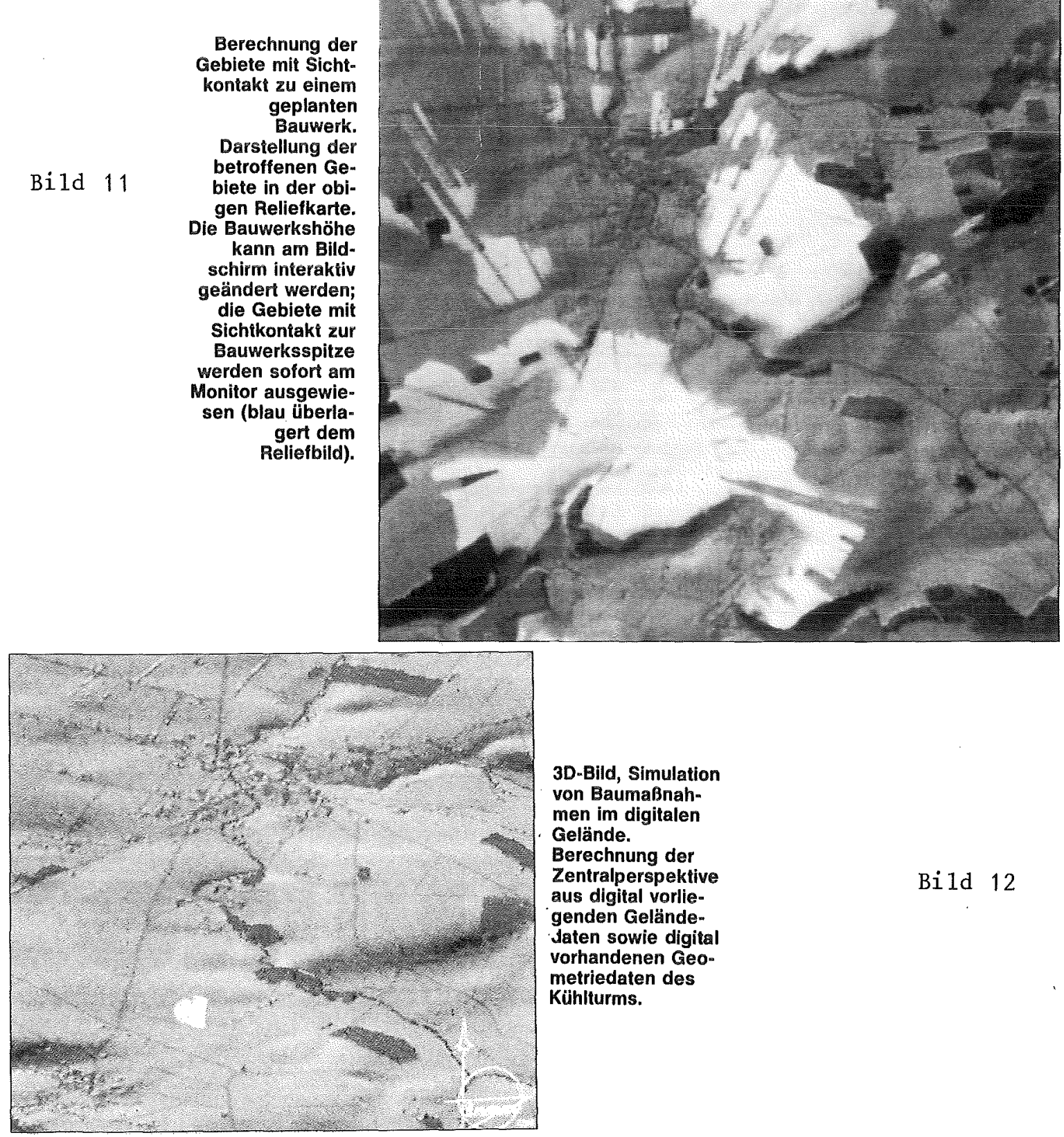

3D-Bild, Simulation

von Baumaßnah-

men im digitalen

Gelände.

Berechnung der

Zentralperspektive

aus digital vorlie-

Bild 12

genden Gelände-

Jaten sowie digital

vorhandenen Geo-

metriedaten des

Kühlturms. 


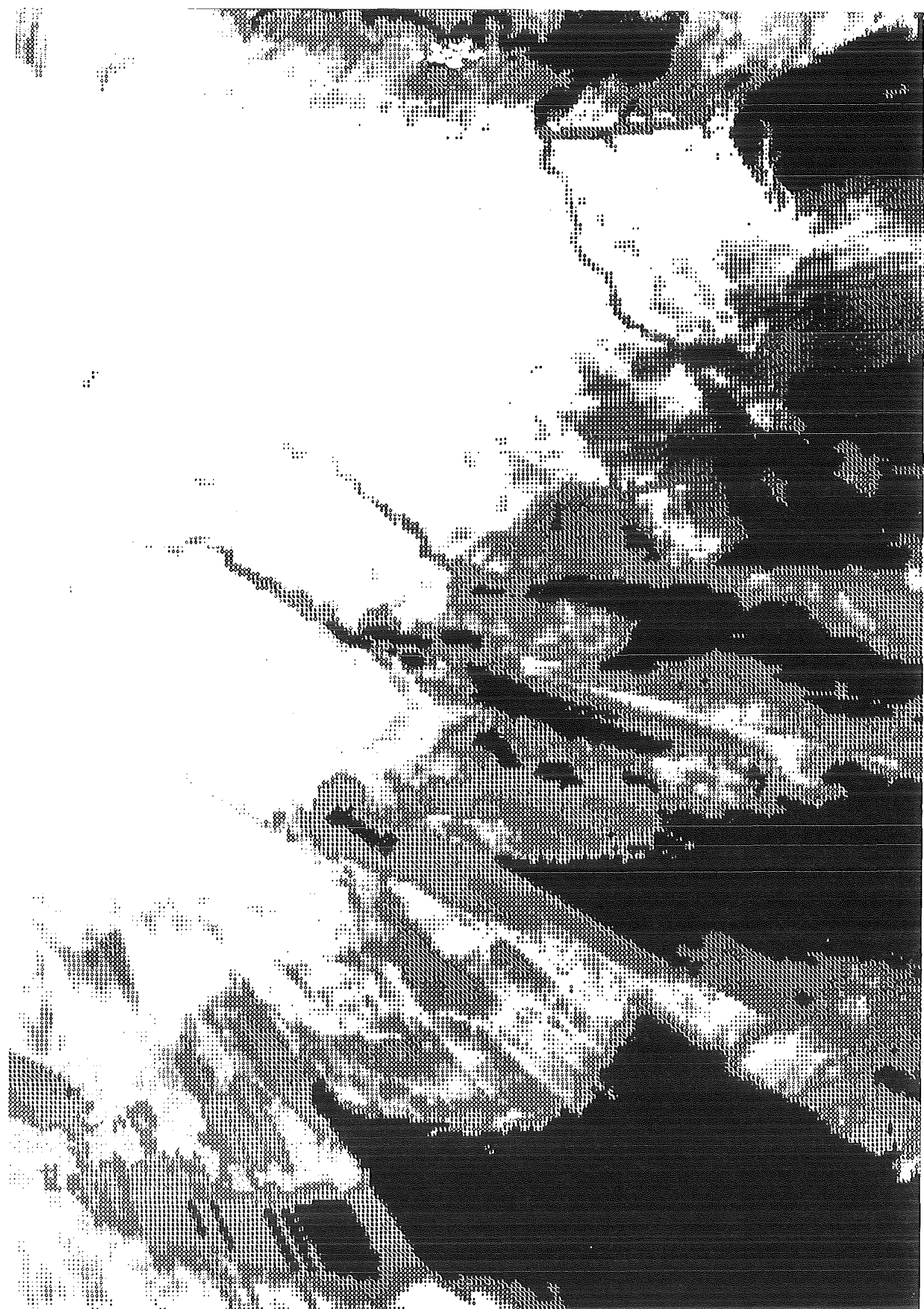

B i 1 d 13

Sichtdiagramm:

Berechnung der visuellen Beeinträchtigung durch Großbauten: die verschiedenen Grauwerte entsprechen den unterschiedlichen Bauwerksbehörden 


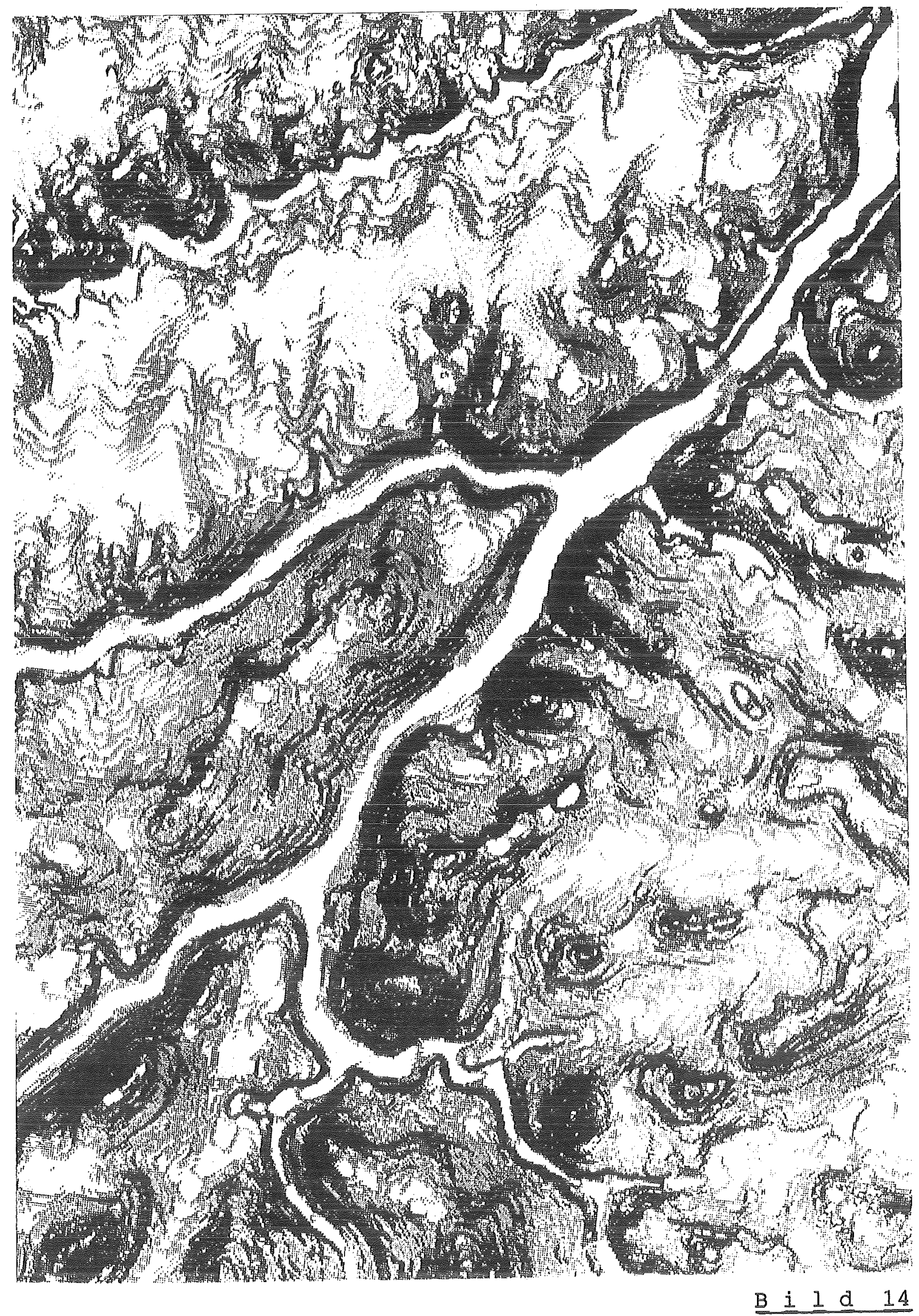

Hangneigungsbild:

starke Schwärzung bedeutet große Hangneigungswinkel 
5. Ausblick:

Der Einzug der digitalen Datenverarbeitung in die moderne Umweltplanung ermöglicht nicht nur Verbesserungen bei der Erfassung, Generalisierung, Fortführung und Neuerstellung von thematischen Umweltkarten, sondern erlaubt mit den hier vorgestellten Methoden und den dann kostengünstig vorliegenden digitalen Daten Planungsaussagen für alle flächenbezogenen Fragestellungen. Beispiele aus der Verkehrs-, Landwirtschafts-, Umwelt- und Katastropeneinsatzplanung wurden hier vorgestelit

Die Zukunft der Umweltplanung wird

- den Einzug von interaktiven Arbeitsplätzen mit Farbdarstellung

- Die Verbindung von Raster- und Vektor-DV

- preiswerte hochauflösende Ausgabemedien

- mehr thematische Umweltkarten

bringen, sowie viele hundert Programme.

Die Universität und Großforschung hat die Aufgabe, diese interessante Entwicklung richtungsweise mitzugestalten und die studenten so auszubilden, daß sie sich dieser modernen Datenverarbeitungsmöglichkeiten virtuos bedienen können (nicht Sklaven der DV werden!).

Gefragt sind Hilfsmittel zur besseren Visualisierung sowie viele spezielle Auswerteprogramme.

Speziell die Verschmelzung von Raster-DV mit Vektor-DV und die Raster-/Vektorkonvertierung sowie die problemlosere vektor-/Rasterkonvertierung werden für die Datenverarbeitung wesentliche Themen sein. Die Geländedatenverarbeitung der IABG arbeitet an diesen Aufgaben. Sie ist zum wissenschaftlichen Gespräch wie auch zur Information über bereits vorhandener Lösungsansätze bereit. 


\section{$\| \mathrm{ABG}$ \\ Geländedatenverarbeitung}

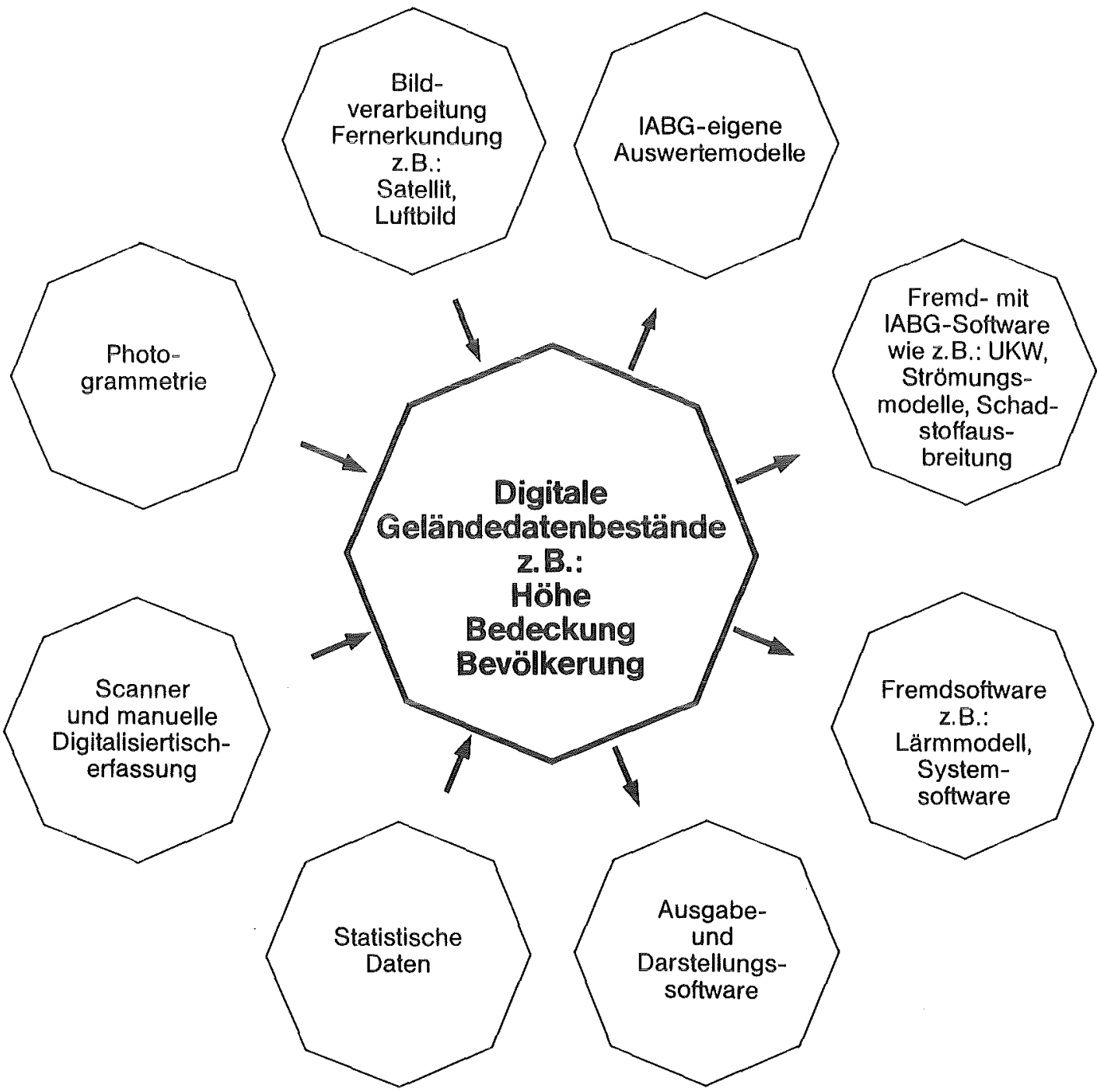

IABG-Rechnerumgebung: Vektorrechner Siemens VP200 (mit

64MB Hauptspeicher) mit Siemens 7.890D,

mit 20 Giga Byte sowie Control Data

CYBER 170-800 Großrechner.

$3 \times$ DEC-VAX mit 4 Giga Byte Plattenspeicher (VAX 780/750/ $\mu$ VAX II) mit 2 VTE-Systemen.

2x Siemens APC-CAD mit je 1 hochauflösendem Farbrasterdisplay (1280 $\times 1024$ RGB).

4 Digitalisierarbeitsplätze mit je einem Farbraster-Kontroll-Monitor. 



\section{Entwicklung eines Informationssystems}

"Unwel tbelastungen und Omwel tprobleme"

E. Reichardt

IABG, ottobrunn

Zusammenfassung:

Der Beitrag befaßt sich mit der Entwicklung eines Informationssystems, das Daten über Umweltbelastungen und Umweltprobleme im Bereich der Bundeswehr bereithät. Ausgehend von den Umwel tbelastungen durch den Betrieb der Bundeswehr und den sich daraus ableitenden Umweltaufgaben wird auf organisatorische Probleme eingegangen, die den Aufbau eines Informationssystems Umwelt vorteilhaft erscheinen 1 ießen. Schließlich wird das Konzept des Informationssystems vorgestellt mit den fachlichen Dateninhalten und den Grundzügen der dv-technischen Realisierung.

\section{Gliederung:}

1. Bundeswehr und Unwelt

- Umweltbelastungen

- Unwel taufgaben

2. Organisatorische Probleme bei der Informationsversorgung

- fachliche Umweltzuständigkeiten

- regionale Zuständigkeiten

3. Konzept des Umweltinformationssystems

- Eachliche Inhalte der Datenbasis

- technische Lösung

4. Ausblick 


\section{Bundeswehr und Omwelt}

\section{Omwel tbelastungen}

Auch in Friedenszeiten, auf die sich die Betrachtung beschränken soll, führt der Dienstbetrieb bei der Bundeswehr zu Belastungen der Umwelt. Beispielhaft für die zahlreichen umweltrelevanten Aktivitäten seien hier genannt:

- die Schießausbildung aller Soldaten

- der militärische Flugbetrieb

- Manöver und Übungen, auch außerhalb des militärischen Geländes

- Pflege, Wartung und Instandsetzung von Kraftfahrzeugen, Schiffen, Flugzeugen etc.

- Erprobung von Waffen und Geräten

Das Spektrum der Umwel tbelastungen umfaßt:

- Iarmemissionen (z.B. Schießlärm, Fluglärm)

- Schadstorfemissionen (z.B. durch Heizanlagen, Fahrzeuge)

- Einleitung von Abwässern (z.B. aus Kasernen, aus dem technischen Bereich)

- Abfälle einschließlich Sonderabfälle (z.B. Altöl, Altreifen, Altbatterien)

- Verwendung von Onwel tchemikalien (z.B. chem. Enteisungsmittel)

- Einwirkungen auf Boden und olkosysteme (z.B. bei Manövern) 


\section{Unwel taufgaben}

Die Umweltbelastungen einerseits und das generell zunehmende Umweltbewußtsein andererseits haben dazu geführt, daß sich auch die Bundeswehr verstärkt mit Umweltfragen auseinandersetzt, wobei die Aufgaben in den letzten Jahren stetig gewachsen sind. Die nachfolgende Aufzählung von Umweltaufgaben erhebt keinen Anspruch auf Vollständigkeit, sollte aber einen Eindruck von Aufgabenumfang und Aufgabenspektrum vermitteln.

- Uberwachung von Anlagen

(Heizanlagen, Tankanlagen, Pipelines, Kläranlagen)

- Ubberwachung der Einhaltung von Rechtsvorschriften

- Planen und Durchführen von Umweltschutzmaßnahmen (technische und organisatorische Maßnahmen)

- Erarbeiten von Vorschriften für den eigenen Bereich (Dienstvorschriften, Erlasse, Durchführungsbestimmungen ...)

- Mitarbeit bei Gesetzesvorhaben (Bund, Länder)

- Öffentlichkeitsarbeit

- Beantwortung von Anfragen, Beschwerden (z.B. parlamentarische Anfragen, Bürgerinitiativen)

- Informieren und Schulen (Militärisches und ziviles Personal)

- Wahrnehmen der Bundeswehrinteressen bei Vorhaben Dritter (z.B. Bauleitplanung)

- internationaler Erfahrungsaustausch

- Einführung der Umweltverträglichkeitsprüfung

Die Vielzahl der Aufgaben hat schließlich in der zweiten Hälfte der $70 e r$ Jahre zur schaffung von stellen geführt, die sich ausschließlich oder überwiegend mit Fragen des Umweltschutzes befassen. Ein großer Teil der $0 . a$. Aufgaben, insbesondere die nicht fachbezogenen Aufgaben, wurden den neuen "fachübergreifenden" Umweltdezernaten in den Wehrbereichsverwaltungen übertragen. Ein fach- und bereichsübergreifendes Referat im Verteidigungsministerium koordiniert und initiiert die Umweltaktivitäten der Bundeswehr insgesamt. Im derzeitigen Ausbaustand sind im Ministerium und bei den Wehrbereichsverwaltungen etwa 15 Beamte und Angestellte ausschließlich für den Umweltschutz eingesetzt.

Zur Bearbeitung der vielfältigen Aufgaben in allen Umweltbereichen werden in den Umweltdezernaten zahlreiche Informationen zur Umweltsituation im eigenen Bereich (z.B. für die offentlichkeitsarbeit, zur Beantwortung von Anfragen, zur Planung von Umweltschutzmaßnahmen) benötigt, deren Beschaffung nicht ohne Probleme ist. 


\section{Organisatorische Probleme bei der Informationsversorgung}

\section{Fachl iche Zustandigkeiten}

Die Schwierigkeiten bei der Informationsbeschaffung liegen zum einen in der breiten streuung der fachlichen Zustandigkeiten für die einzelnen Teilaspekte des Umweltschutzes. So ist z.B. die Truppe (Teilstreitkräfte Heer, Luftwaffe, Marine) verantwortlich für

- den Einsatz der Fahrzeuge

- den Einsatz der Kraft- und Schmierstoffe

- Manöver und úbungen

- Flugplatzenteisung (chem. Enteisungsmittel, nur Luftwaffe)

- Flugbetrieb und Schießbetrieb

in ihrem Bereich, und für die damit zusammenhängenden Umweltbelastungen.

Der organisatorisch von der Truppe unabhängige Vermaltungsbereich ist zuständig für

- Bau und Betrieb der Heizanlagen (insbesondere für die Truppenunterkünfte)

- Bau und Betrieb von Tankanlagen für Kraftstoffe (aber die Verantwortung für den Verbrauch liect bei der Truppe)

- Bau und Betrieb von Anlagen zur Abwasserentsorgung (Erzeuger des Abwassers ist in erster Linie die Truppe)

- Abfallbeseitigung einschl. Sonderabfall (einschließlich des von der Truppe "produzierten" Abfalles)

- Betrieb bundeswehreigener Deponien

und die damit zusammenhängenden Belastungen der Umwelt.

Erprobungen von Wehrmaterial auch aus Umweltsicht sowie einige fachwissenschaftliche Detailuntersuchungen aus den Bereichen Gewässerschutz und Bodenschutz werden im Rüstungsbereich durchgeführt, der organisatorisch wiederum getrennt vom Verwaltungsbereich bzw. von der Truppe zu sehen ist.

Bei der Festlegung dieser Zuständigkeiten standen andere Aufgaben im Vordergrund. Fragen des Umweltschutzes wurden entweder noch gar nicht beachtet, oder sie mußten den primären zielsetzungen (Verteidigungsauftrag) untergeordnet werden. 


\section{Regionale Zuständigkeiten}

Erschwerend für die Beschaffung der Umweltinformationen kommt hinzu, daß die Informationen, die bei den verschiedenen Teilbereichen der Bundeswehr verfügbar sind, auch regional breit gestreut sind. Dies soll hier nur am Beispiel des Verwaltungsbereiches demonstriert werden, gilt aber in ähnlicher weise auch für den Truppenbereich.

Die Bundeswehrverwaltung wird in der Region durch die sechs Wehrbereichsverwaltungen repräsentiext, die von der Fläche her etwa die Größe eines großen Bundeslandes betreuen. Den Wehrbereichsverwaltungen sind wiederum die insgesamt 184 standortverwaltungen unterstellt, die alle Bundeswehr-Liegenschatten wie z.B. Kasernen, Ubungsplätze, Flugplätze oder Schießplätze verwalten, die sich in ihrem Zuständigkeitsbereich befinden.

Daten zu Umweltbelastungen betreffen, zumindest im Verwaltungsbereich, in erster Linie eine einzelne Liegenschaft. Zur Beantwortung von Anfragen oder Beschwerden, die in der Regel eine bestimmte Liegenschaft betreffen, sind die Referate/Dezernate des fachübergreifenden Umweltschutzes auf telefonische oder schriftliche Auskünte der jeweiligen Standortverwaltung angewiesen.

Noch schwieriger gestaltet sich für die Referate des fachübergreifenden Umweltschutzes die Erstellung einer Gesamtschau, etwa für einen Umweltbereich wie Luftreinhaltung und einen bestimmtem Wehrbereich. Hierzu müßten die Daten aus allen betroffenen Standortverwaltungen zusammengetragen werden, ein Aufwand, der bisher derartige systematische Zusammenstellungen fast vollständig verhindert hat.

Um die Arbeiten auf dem Gebiet des Umweltschutzes künftig hinsichtlich der Informationsversorgung besser unterstützen zu können, wurde beschlossen, die wichtigsten Daten zu Umweltbelastungen im Bundeswehrbereich systematisch zu erheben bzw. zu aktualisieren und sie den Referaten/ Dezernaten des fachübergreifenden Umweltschutzes in Form eines Informationssystems zur Verfügung $\mathrm{zu}$ stellen. 


\section{Ronzept des Informationssystems Onwelt}

Die Lösung geht aus von einer eigenständigen Datenbank Umwelt, deren struktur und Dateninhalte sich primär an Umweltgesichtspunkten orientieren. Die Daten werden soweit wie möglich aus anderen bereits existierenden oder in Aufbau bzw. Planung befindIichen Fachinformationssystemen der Bundeswehr übernommen. Hierzu müssen diese Fachinformationssysteme um die Umweltdatenkategorien ergänzt werden. Erfassung und Aktualisierung dieser Daten liegen dann in der Verantwortung der Betreiber dieser Fachinformationssysteme, so daß bei den Dezernaten des fachübergreifenden Umweltschutzes keine Kapazitätsengpässe entstehen. Eine eigene Erfassung muß dann nur noch für die Daten aufgebaut werden, die nicht über eines der Informationssysteme erhältlich sind wie z.B. Daten über Beschwerden.

Die Datenbank Umwelt wird zentral beim Großrechner im Verteidigungsministerium aufgebaut, es sind Zugriffsmöglichkeiten für die Umweltreferate im Ministerium selbst sowie auch für die Umweltdezernate in den sechs wehrbereichen vorgesehen. In einigen Umweltdezernaten sollen PC's eingesetzt werden, dort können die Daten dann auch lokal zwischengespeichert werden.

Die Verarbeitung i.e.s. Wird in die Auswertungen des Datenbestandes verlagert, hier soll also das Zahlenmaterial entsprechend den Vorstellungen der umweltfachleute aufbereitet und analysiert werden. Hierzu gehört auch die Erstellung von Tabellen und Grafiken, die Einarbeitung der Daten in Antwortschreiben und Berichte.

\section{Fachbezogene Inhalte der Datenbasis}

Die gespeicherten Dateninhalte orientieren sich primär an Umweltgesichtspunkten, so wie sie sich aus der Aufgabenstellung der Umweltreferate/ -dezernate ergeben. Aus fachlicher sicht lassen sich folgende Teilgebiete unterscheiden:

- Lärmbekämpfung

- Luftreinhaltung

- Gewässerschutz und Wasserwirtschaft

- Abfallwirtschaft

- Bodenschutz, Naturschutz und Landschaftspflege

Die Frage, welche Dateninhalte im einzelnen vorgehalten werden, soll hier am Beispiel des Bereiches Abfallwirtschaft behandelt werden. In diesem Teilgebiet sind drei Tabellen vorgesehen für 
- Abfallbeseitigung

- Wiederverwertung/ Recycling

- bundeswehreigene Deponien

Die Tabelle "Abrallbeseitigung" sieht je einen Eintrag vor pro Liegenschaft und beseitigter Abfallart mit folgenden Datenkategorien: Abfallart, Abfallbeförderer, Abfallbeseitiger, Abfallnachweis gem. AbfNachwV, Abfallmenge im abgelaufenen Kalenderjahr, Kosten der Beseitigung, Sammelstellen in der Liegenschaft, Vorbehandlung/Vorsortierung in der Liegenschaft.

Die Tabelle "Wiederverwertung/Recycling" sieht je einen Eintrag pro tiegenschaft und Verwertungsgut vor mit den folgenden Dateninhalten: Verwertungsgut (LAGA-Schlüssel), Verbleib, Menge im abgelaufenen Kalenderjahr, Kosten/Erlöse, Sammelstellen in der Liegenschaft, Vorbehandlung/Vorsortierung.

Die Tabel le "Bundeswehreigene Deponien" umfaßt alle noch von der Bundeswehr betriebenen sowie alle stillgelegten Deponien mit folgenden Daten: Jahr der Eröffnung, Jahr der Schließung, Deponievolumen, Deponiefläche, Deponieinhalt, Bodenbeschaffenheit, Sickerwasserentsorgung, Deponiezustand, Deponieüberwachung, Umweltrisiken.

\section{Dv-technische Realisierung}

Das Informationssystem ist derzeit in der Realisierungsphase. Aus dv-technischer Sicht sind die folgenden Realisierungscharakteristika erwähnenswert:

- Aufbau der Datenbank Umwelt auf dem zentralen Rechner im Verteidigungsministerium unter dem DBMS DB2.

- über Standleitungen sind Terminals bzw. PC's im Ministerium und in den Wehrbereichsverwaltungen angeschlossen. Für die Auswertungen des Datenbestandes stehen die Abfragesprachen von DB2 zur Verfügung. Für weitergehende Aufbereitungen werden einfache Grafik-Software (für sog. Business-Grafiken) und einfache Textbearbeitungssoftware eingesetzt.

- Erfassung und Aktualisierung erfolgt soweit wie möglich über die bereits bestehenden Fachinformationssysteme. Um die Daten dann in das Informationssystem Umwelt übernehmen zu können, sind wegen der unterschiedlichen Datensichten und der unterschiedlichen DBMS, die jeweils zum Einsatz kommen, eine Reihe von Transformationsschritten notwendig.

- für die Erfassung und Aktualisierung der Daten, die nicht über Fachinformationssysteme erhältlich sind, ist die Möglichkeit einer dezentralen Erfassung und Ubernahme auf die Datenbank Umwelt vorgesehen. 


\section{Ausblick}

Abschließend sol1 noch auf einige Ausbauwünsche und Ausbaumöglichkeiten eingegangen werden.

Derzeit können Umweltdaten nur aus einem Fachinformationssystem übernommen werden, nämlich aus dem System, das die Daten aus der Zuständigkeit des Verwaltungsbereichs (d.h. zu den einzelnen Liegenschaften) vorhält. Genauso wichtig sind aus der sicht des Umweltschutzes auch die Daten zu umweltbelastungen, die in den Verantwortungsbereich der Truppe (Teilstreitkräfte Heer, Luftwaffe und Marine sowie Sanitätswesen) fallen. Benötigt werden hier vor allem Daten zu Manövern und úbungen. Informationssysteme $\mathrm{zu}$ diesen Gebieten sind aber derzeit noch im Stadium der Entwicklung, so daß über eine Datenübernahme erst zu einem späteren zeitpunkt entschieden werden kann.

Die Dezernate/Referate des fachübergreifenden Umweltschutzes benötigen furr ihre Arbeiten auch Umweltinformationen aus dem nichtmilitärischen Bereich. In erster Linie sind hier die Entwicklungen auf den Gebieten Orweltrecht und Orwelttechnik $z u$ nennen. Das derzeitige Angebot der öffentlich zugänglichen Datenbanken deckt sich allerdings noch nicht mit dem Bedarf. Da die Entwicklung in diesem Bereich sehr dynamisch ist, wird sie jedoch aufmerksam verfolgt. Falls auf dem Sektor Umweltrecht in absehbarer Zeit keine geeignete externe Datenbank zur Verfügung stehen sollte, wäre auch der Aufbau eines eigenen Auskunftsystems zu prüfen.

Die in der Umweltdatenbank vorgehaltenen Daten, müssen sehr häufig in Berichte oder Antwortschreiben eingearbeitet werden. Aus diesem Grund sollten die Möglichkeiten der Bearbeitung von Texten weiter ausgebaut werden. Für Planungs- und Kontrollarbeiten sind oft umfangreiche Tabellen zu erstellen, deren Inhalt von Fall zu Fall wechselt. Zur Unterstützung sollten hier Tabellenkalkulationsprogramme eingesetzt werden, wie sie aus dem PC-Bereich hinreichend bekannt sind.

Der derzeitige Ausbau des Systems sieht eine Grafik-Unterstützung nur bei der Erstellung von Business-Grafiken vor. Für die Belange der Umweltdezernate ist darüber hinaus eine Darstellung von Umweltdaten in Kartenform erforderlich (z.B. für die Darstellung von Schutzgebieten aller Art, für Lagepläne und Bebauungspläne oder für die Darstellung geplanter Bewegungen bei Ubungen oder Manövern). Auch hier sollen gegebenenfalls in einer späteren Ausbaustufe die hard- und softwareseitigen Voraussetzungen für die Erstellung und Ausgabe derartiger Grafiken auf dem Rechner geschaffen werden.

Die Einsatzmöglichkeiten von Expertensystemen zur Unterstützung der Axbeiten in den Umweltdezernaten werden derzeit geprürt. Auf dem Gebiet der Umweltverträglichkeitsprüfung zeigen sich einige Ansatzpunkte.

Wichtiger als eine der 0.a. Erweiterungen ist allerdings, daß nach Fertigstellung des Systems die Mitarbeiter in den Umweltreferaten, die in der Regel bisher kaum Erfahrungen im Umgang mit der Datenverarbeitung vorweisen können, lernen, mit dem System umzugehen und ihre Auskunfts- und Verarbeitungwünsche umzusetzen. 


\title{
Rechnergestützte Kontrollsysteme für Sonderabfälle \\ - Anforderungen und Realisierungsbedingungen -
}

\author{
Dipl-Phys. D. Brune \\ Kernforschungszentrum Karlsruhe $\mathrm{GmbH}$ \\ Abteilung für Angewandte Systemanalyse (AFAS) \\ Postfach 36 40, D-7500 Karlsruhe 1
}

\section{Zusammenfassung:}

Vor dem Hintergrund der derzeitigen unbefriedigenden Möglichkeit der Kontrolle von Sonderabfällen und der Identifikation ihrer Inhaltsstoffe werden Vorschläge zur Steigerung der Effektivität der Überwachungstechniken gemacht. Diese beziehen sich auf eine Ausweitung der Auskunftspflicht der Produzenten über Schadstoffgehalt von Produkten und Abfällen und deren Erfassung durch rechnergestützte Kontrollsysteme. Zur Erhöhung der Akzeptanz dieser zusätzlichen staatlichen Eingriffe sollen den Auskunftspflichtigen verschiedene rechnergestützte Informationssysteme angeboten werden.

\section{Gliederung:}

1. Einleitung

2. Beschreibung der derzeitigen Situation

3. Vorschläge

4. Literatur 


\section{Einleitung}

Die Behandlung und die Beseitigung von Abfällen stellt ein immer noch wachsendes Problem von besonderer Größe und Komplexität dar. Zahlreiche Störfälle in Behandlungsanlagen, schädliche Auswirkungen auf die Umwelt von Emissionen aus Deponien und Müllverbrennungsanlagen, die nur unzureichenden Möglichkeiten der Abgas- und Abwasserreinigung aufweisen, aber auch die schleichende Vergiftung von Boden und Grundwasser durch nur geringfügig kontaminierte Abfälle wie Klärschlämme, Baggergut und Abwasser aus Kläranlagen ohne weitgehende Reinigungsstufen sind Ausdruck des Ungenügens bisheriger Lösungsversuche. Eine große Zahl von Altablagerungen und früheren Industriestandorten (die sogenannten "Altlasten"), bei denen nur unzureichende Kenntnisse über die dort gelagerten Abfälle und Reststoffe bestehen, verschärft das Problem. Ein besonderes Gefahrenpotential stellen dabei die Sonderabfälle aus dem Bereich des Produzierenden Gewerbes und der Krankenhäuser und neuerdings auch aus dem Hausmüllbereich dar. Ihre vollständige Erfassung und Überwachung von der Entstehung bis zur schadlosen Beseitigung ist zur Vermeidung von Umweltschäden und Gesundheitsgefahren für Menschen, Tiere und Pflanzen unerläßlich.

\section{Beschreibung der derzeitigen Situation}

Die bisherigen gesetzlichen Regelungen zur Erfassung, zur Überwachung des Transports und zur Kontrolle der Behandlung/Beseitigung sind ergänzungsbedürftig. Dies ergibt sich schon aus der Tatsache, daß eine einheitliche Definition nicht existiert. Nach dem üblichen Sprachgebrauch sind Sonderabfälle diejenigen Abfälle, die

- nicht mit Hausmüll zusammen beseitigt werden können, gefährlich (toxisch, explosibel, brennbar u.a.m.) sind, und an deren Beseitigung besondere Anforderungen gestellt werden müssen ( $\$ 2 \mathrm{Abs} .2 \mathrm{AbfG}$ ).

Demnach werden so unterschiedliche Abfälle wie Bauschutt und Galvanikschlämme als Sonderabfälle bezeichnet.

Die Feststellung der Gefährlichkeit der Sonderabfälle erfolgt in der Bundesrepublik Deutschland durch Beschreibung des Abfalltyps, der Herkunft und der Inhaltsstoffe, die auf die Gefährlichkeit schließen lassen. Ein Beispiel: 
Abfallart: Ofenausbruch aus metallurgischen Prozessen

Eigenschaften: $\quad$ arsen-, blei-, cadmium-, cyanid-, quecksilberhaltig

Abfallschlüsselnummer: 31108

Herkunft: $\quad$ NE-Metallerzeugung, Hochofenwerke.

Insgesamt sind auf diese Weise 98 Abfallarten gekennzeichnet. In anderen Ländern werden andere Prüfkritierien verwandt, z.B. Beschreibung der Abfälle mittels

Testverfahren (Reaktionsverhalten auf unterschiedliche Einflüsse und $\mathrm{Be}$ lastungen),

Analyse der chemischen Inhaltsstoffe und Festlegung bestimmter Höchstkonzentrationen.

Das Verfahren in der Bundesrepublik Deutschland ist pragmatisch und läßt sich gut handhaben. Es ermöglicht aber nicht die Identifikation von gefährlichen Verbindungen wie z.B. Dioxin, die bei unterschiedlichen Verfahrensschritten entstehen können. Auch bei den anderen Prüfmethoden ist die Genauigkeit der Abfallkennzeichnung von der Schärfe des "analytischen Fensters" abhängig.

Die Überwachung des Transports und der Beseitigung der Sonderabfälle unterliegt in der Bundesrepublik Deutschland dem sogenannten Begleitscheinverfahren. Abfallerzeuger, Einsammler und Beförderer von Abfällen sowie Betreiber von Abfallbeseitigungsanlagen sind zur Führung von Nachweisbüchern, d.h. Sammlungen von Begleitscheinen für jeden Abfalltransport und jede Abfallart verpflichtet. Die Begleitscheine enthalten Angaben zur Abfallmenge, -art und konsistenz und bestehen aus 6 Ausfertigungen, von denen jeweils zwei Ausfertigungen beim Abfallerzeuger und bei der überwachenden Behörde verbleiben, jeweils eine Ausfertigung beim Beförderer und Abfallempfänger. Bei jeder Übergabe der Abfälle wird eine Ausfertigung der überwachenden Behörde übersandt (spätestens 10 Tage nach der Übergabe). Der Abfallempfänger übersendet auch eine Ausfertigung dem Abfallerzeuger für dessen Nachweis einer sachgerechten Entsorgung.

Dieses Verfahren hat verschiedene Nachteile. Es ist immer möglich, die Sonderabfälle vollständig aus dem Geltungsbereich des Abfallbeseitigungsgesetzes herauszubringen (und damit von den oben beschriebenen Kontrollmaßnahmen zu befreien), indem sie zu Wirtschaftsgütern deklariert werden. Eine Kontrolle über 
die tatsächliche Verwertung ist nicht vorgesehen. Als Wirtschaftsgüter können sie auch einfacher ins Ausland verschoben werden und dort gegebenenfalls verwertet oder auch einer kostengünstigeren Beseitigung zugeführt werden. Weiterhin ist nicht auszuschließen, daß Abfallerzeuger gefährliche Abfälle mit Inertmaterial zu "harmlosen" Abfällen verschneiden, die dann nicht mehr als Sonderabfälle deklariert werden müssen (für Transporteur und Beseitiger ist dies inzwischen verboten). Schließlich sagen die Deklarationen nicht immer genügend über die Inhaltsstoffe aus. Dies gilt insbesondere für die Angabe von Giftstoffen, wie eine Reihe von Organochlorverbindungen, die nach dem derzeitigen Deklarierungsmodus nicht erfaßt werden.

\section{Vorschläge zur Verbesserung der Schadstoff-Flußkontrolle}

Eine genaue Kenntnis der Beschaffenheit und der Inhaltsstoffe der Sonderabfälle sowie von Produkten, die nach ihrem bestimmungsgemäßen Gebrauch zu Abfällen werden, ist aber erforderlich, wenn

- Verbrennungsanlagen und Deponien (oder andere Behandlungsanlagen) ohne Schädigung der Umwelt und sicher betrieben werden sollen,

sie verstärkt einer Verwertung zugeführt werden sollen,

- die Entstehung von Schadstoffen und ihr Eintrag in Produkte sowie ihr Verbleib kontrolliert und nach Möglichkeit bilanziert werden sollen.

Eine Möglichkeit zur Verbesserung der Situation besteht im Einsatz rechnergestützter Kontrollsysteme. Dies gilt schon für das derzeitige Begleitscheinsystem, wenn das Übeprüfen und Zusammenführen der Begleitscheine mit Hilfe von Datenverarbeitungsanlagen erfolgt.

Erhebliche Verbesserungen lassen sich aber dann erwarten, wenn die gesetzlichen und technischen Voraussetzungen für eine weitgehende Kontrolle von Stoffflüssen mit der Möglichkeit der Erstellung von Stoffbilanzen geschaffen werden. Dies setzt allerdings eine Änderung des Abfallbeseitigungsgesetzes voraus, da Stofferzeugungs- und Stoffumwandlungsprozesse sowie Produktionsverfahren beim Abfallerzeuger miterfaßt werden müßten. Die ausschließliche Kennzeichnung der Abfälle durch Deklarierung gemäß einem vorgegebenen Katalag ist unzureichend. Möglicherweise könnten die Vorschriften der 12. Verordnung zur Durchführung des Bundesimmissionsschutzgesetzes (Störfallverordnung) und die zugehörigen Verwaltungsvorschriften als Beispiele für eine solche Gesetzesänderung herangezogen werden, da hier schon weitreichende Auskunftspflichten 
für Betriebe, in denen mit gefährlichen Stoffen gearbeitet wird, festgeschrieben sind.

Zweifellos würde eine solche Ausdehnung der Auskunftspflicht von den Betroffenen als zusätzlicher staatlicher Eingriff aufgefaßt werden. Möglicherweise muß auch mit Widerständen oder zumindest mit schleppender Befolgung der Vorschriften gerechnet werden. Es ist daher geboten, die Akzeptanz dieser (vorgeschlagenen) Maßnahmen durch zusätzliche Angebote zu erhöhen. Diese Angebote könnten in verschiedenen Dienstleistungen bestehen, die den Auskunftspflichtigen bei der Abgabe ihrer Erklärung bereitgestellt würden. Denkbar ist

- die Umwandlung des Meldesystems in ein Informationssystem für potentielle Verwerter ("elektronische Abfallbörse")

- ein Informationssystem ("Expertensystem") über Vermeidungs-, Behandlungs- und Beseitigungsmöglichkeiten,

eine "Clearing-Stelle", bei der Anforderungen auf Abfallbeseitigung so auf bestehende Behandlungs- und Beiseitigungsanlagen verteilt werden, daß ein gesamtwirtschaftliches und ökologisches Optimum entsteht.

Es ist klar, daß dies nur mit Hilfe der elektronischem Datenverarbeitung durchführbar ist. andererseits ist aber auch die Bereitschaft zur Mitarbeit der Betroffenen unerläßlich. Dies ist um so wahrscheinlicher, je mehr wirtschaftliche Vorteile für den einzelnen Auskunftspflichtigen erkennbar sind. Gelingt diese Kooperation, so könnte dieses System von Auskunftspflicht und angebotener Information einen effektiveren Beitrag zur Lösung des Sonderabfallproblems leisten als die bisherigen Kontrollsysteme.

\section{Literatur:}

Schenkel, W.:

Ist die geordnete Beseitigung gefährlicher Abfälle eine Utopie? Müll und Abfall 5 (1984), S. 14-122

\section{Bundesregierung:}

Abfallgesetz (AbfG) vom 27. August 1986, BGBL I (1986), S. „410

Abfallnachweis-Verordnung (AbfNachwV.) vom 2. Juni 1978, BGBI I (1978), S. 668. Verordnung zur Bestimmung von Abfällen nach $\$ 2$ Abs. 2 des Abfallbeseitigungsgesetzes vom 24. Mai 1977, BGBL I (1977), S. 773

Zwölfte Verordnung zur Durchführung des Bundes-Immissionsschutzgesetzes (Störfallverordnung) - 12. BIm,SchV vom 27. Juni 1980, BGBL I (1980), S.772 geändert durch VO vom 24. Juni 1985, BGBL I (1985), S. 1603. 

Überwachungssysteme in der Abfallbehandlung

\author{
R. Weidemann, R. Kerpe, A. Jaeschke \\ Kernforschungszentrum Karlsruhe $\mathrm{GmbH}$ \\ Institut für Datenverarbeitung in der Technik \\ Postfach 3640, 7500 Karlsruhe
}

\title{
Zusammenfassung
}

Überwachungssysteme in der Abfallbehandlung sind in ihren Grundzügen Materialverfolgungssysteme oder basieren zumindest auf solchen Systemen. Sie überdecken die Bereiche Abfall-Erzeugung, -Transport, -Behandlung, -Zwischenlagerung, -Deponierung.

Anhand von Überwachungssystemen für Kernmaterial (System K2) und radioaktive Reststoffe (System KADABRA), die vom IDT konzipiert und realisiert wurden und im KfK betrieben werden, wird das Vorgehen beim Entwurf und der prinzipielle Aufbau solcher Systeme gezeigt, sowie ein übertragbares Konzept dargestellt, das z. B. auch auf die Überwachung in der Materialverfolgung und Bilanzierung bei konventionellem Abfall anpaßbar ist.

Die Vorteile einer Lösung, die betriebliche und behördliche Überwachung integriert und die Erfahrung beim Betrieb der o. g. Systeme, wird diskutiert.

\section{Gliederung}

1. Einführung

2. Das Kernmaterialüberwachungssystem K2

3. Das System KADABRA

3.1 Die Aufgabe

3.2 Das Modell

3.3 Die Realisierung

4. Die Übertragbarkeit des Konzepts

5. Literaturverzeichnis 


\section{Einführung}

Überwachungssysteme in der Abfallbehandlung sind in ihren Grundzügen Materialverfolgungssysteme oder basieren zumindest auf solchen Systemen. Sie überdecken die Bereiche Abfall-Erzeugung, -Transport, -Behandlung, -Zwischenlagerung, -Deponierung

Zu unterscheiden ist die betriebliche und behördliche Überwachung.

Die betriebliche Überwachung geschieht in der Regie des Betreibers selbst mit den Zielen:

- Eigenkontrolle der behördlichen Auflagen,

- Produktionsüberwachung,

- Prozeßoptimierung unter kommerziellen Aspekten,

- Betriebsplanung etc.

Die behördliche Überwachung besteht in einer externen Betreiber-unabhängigen Kontrolle vorgegebener Auflagen. Die behördliche Überwachung geschieht anhand von:

- Grenzwerten für Stoffmerkmale (phys. chem. Eigenschaften),

- Bilanzen in den Stoffströmen und

- Grenzwerten (Auflagen) für Anlagen und Geräte bei Transport, Behandlung und Lagerung.

Entsprechend sind in der Überwachung relevant:

- die einer Abfall-Einheit zugeordneten Materialdaten; das schließt ein:

- Mengenangaben,

- physikalische Beschreibung,

- chemische Zusammensetzung etc.

- die ebenfalls einer Abfall-Einheit zugeordneten Daten zur Historie und zur Behandlung des Items; das sind Angaben wie:

- Herkunft,

- Transport,

- Verarbeitungsprozesse,

- Lagerung (Verbleib) etc.

und

- die einer Verarbeitungsanlage oder einem -gerät zugeordneten Daten, wie z.B.:

- Durchsatz

- Rohstoff-, Energieverbrauch

- Betriebs- und Ausfallzeiten (Störungen) etc. 
Das ganz allgemein bei Materialflußproblemen von Aufsichtsinstanzen angewendete Überwachungsverfahren basiert auf zwei Maßnahmen:

- turnusmäßige oder ereignisgesteuerte Meldung des Betreibers an die Kontrollinstanz und

- regelmäßige oder sporadische Verifikation der Meldungen meist anhand von Stichproben durch die Aufsichtsinstanz beim Betreiber (Inspektion).

Die Meldungen werden meist über formalisierte Berichte abgewickelt, die manuell oder mit DVUnterstützung erstellt werden. Die für die Meldung notwendigen Daten können speziell erfaßt oder aus betrieblichen Prozeßinformations- bzw. -automationssystemen abgeleitet werden. Letzteres hat wesentliche Vorteile bezüglich

- Akzeptanz, da kein zusätzlicher Erfassungsaufwand entsteht,

- Datenzuverlässigkeit; durch Konsistenz zum betrieblichen System,

- Sicherheit gegen gezielte Verfälschung,

- Möglichkeit für ein real-time Meldewesen.

Optimal erscheint daher ein Überwachungssystem, das in Form einer Schicht dem betrieblichen Informationssystem überlagert ist und mit diesem eine gemeinsame Bedieneroberfläche hat, die betrieblich orientiert ist und die behördliche Überwachungskomponente für den Bediener verdeckt.

Systeme dieser Art wurden vom Institut für Datenverarbeitung in der Technik (IDT) für unterschiedlichste Anwendungen schon mehrfach konzipiert, realisiert und installiert.

Anhand zweier Beispiele soll im folgenden auf spezielle Charakteristika eingegangen werden, die bei Entwicklung und Betrieb solcher Überwachungssysteme von entscheidender Bedeutung sind:

1. Kernmaterialüberwachungs-System (KMÜ) K2/1/ für das Kernforschungszentrum Karlsruhe (KfK) und

2. Das System KADABRA 12,3 , ein Informationssystem für die Behandlung von radioaktiven Reststoffen in der KfK

\section{Das Kernmaterialüberwachunqssystem K2}

Diese Anwendung ist nicht im Bereich Abfall angesiedelt; da jedoch die gleichen Grundprinzipien in der Überwachung gelten, kann sie als Beispiel herangezogen werden.

Spaltbares Kernmaterial (KM) unterliegt in der BRD der Überwachung internationaler Behörden (EURATOM, IAEA), die diese Funktion auf der Basis eines formalisierten Berichtswesens und von 
Inspektionen beim Betreiber ausüben. Beides setzt eine lückenlose Buchführung über das bei dem Betreiber vorhandenen KM und alle damit durchgeführten phys./chem. Veränderungen voraus.

Diese Buchführung wird im KfK mit dem KMÜ-System, K2 durchgeführt. Das System ist als verteiltes System konzipiert (Abb. 1). In allen Bereichen, die mit KM umgehen, sind PCs installiert. Die Buchungen erfolgen über die Bedienperipherie der PCS. Die PCs sind sternförmig an den Großrechner der KfK gekoppelt, der aber lediglich der Kommunikation zwischen den PCs und der Datensicherung dient.

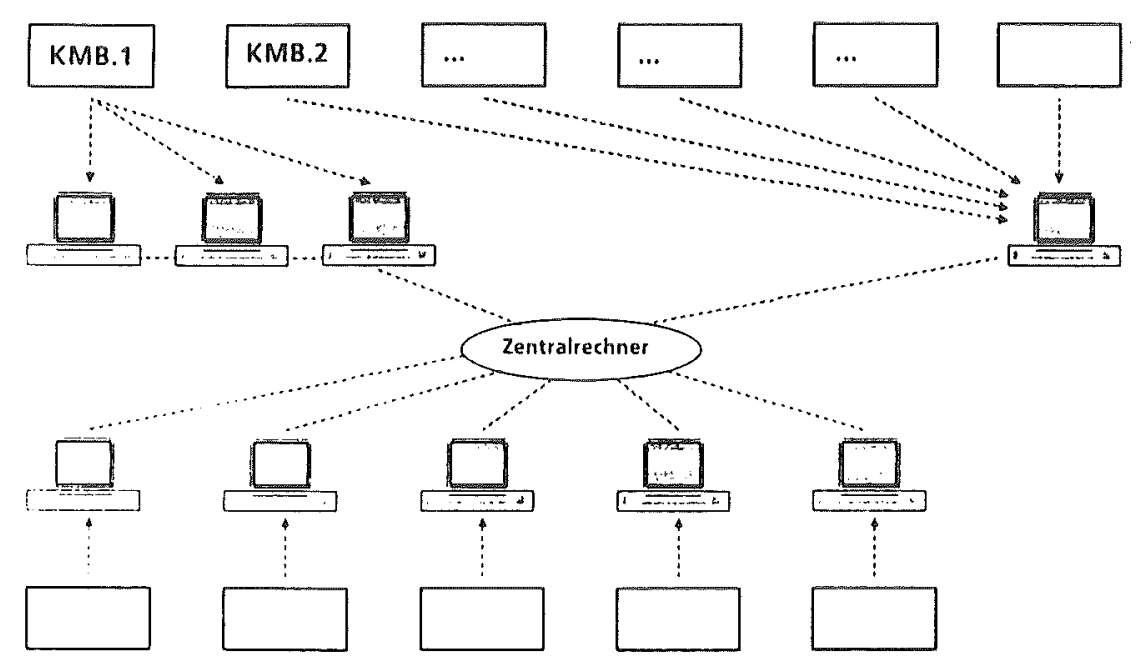

Abb. 1: Konfiguration des K2-Systems der KfK $(\mathrm{KMB}=$ Kernmaterialbereich)

Im vorliegenden Zusammenhang interessant ist die Struktur der Anwendungssoftware im PC. Hier ist die Trennung in eine der behördlichen Überwachung dienenden Komponente und eine Komponente, die das betriebliche KM-Management unterstützt, konsequent durchgeführt.

Die behördliche Überwachungskomponente ist bei allen Anwendern identisch. Sie besitzt ihr eigenes Dialogsystem zur Erfassung aller zur Überwachung notwendigen Daten, die Module zur Datenverarbeitung und Systemverwaltung und die Module zur Erstellung der Berichts- und Inspektionslisten für die Überwachungsinstanzen. Diese Komponente mit ihrer E/A-Oberfläche ist autark anwendbar bei allen Anwendern, die kein eigenes betriebliches KM-Management benötigen.

Existiert eine betrieblich orientierte Komponente, so ist diese anlagenspezifisch und von Anwender zu Anwender unterschiedlich, was die Erfassungsdialoge und die betrieblich benötigten Berichte und Informationsausgaben betrifft. Das Konzept sieht vor, daß in der betrieblichen Komponente die für die behördliche Überwachung erforderlichen Daten selektiert und in die 
behördliche Komponente transferiert werden, wodurch in dieser Komponente einige (im Idealfall alle) der Erfassungsdialoge wegfallen können. Im letzten Fall ist die angestrebte Struktur mit zwei Schichten und einer betrieblich orientierten Bedieneroberfläche erreicht, wobei die behördliche Komponente auf der Datenerfassungsseite vollständig verdeckt wird.

Bei der Konzeption der beiden Komponenten zur betrieblichen und behördlichen Überwachung ergibt sich ein generelles Problem, da an die zwei Komponenten sehr unterschiedliche Anforderungen gestellt werden.

Während die Anforderungen der behördlichen Überwachung relativ starr sind, mit dem Hauptziel zuverlässiger und vollständiger Daten, sind die Anforderungen an die Komponenten der betrieblichen Überwachung Flexibilität, Erweiterbarkeit und Anpaßbarkeit an betrieblich bedingte Anderungen von Anlagen und Abläufen. Dies kann in Realisierung und Betrieb zu Problemen führen, wenn nicht

- das Interface zwischen den Komponenten eindeutig definiert ist und

- die Struktur auch der betrieblichen Komponente einfach und überschaubar ist und dies auch bei Modifikationen in der Komponente bleibt.

Wie solche Strukturen aus den Gegebenheiten der Prozeßanlage und dem vorgegebenen Anforderungsspektrum ableitbar sind, sei am Beispiel des betrieblich/behördlichen Überwachungssystems KADABRA für die Behandlung radioaktiver Reststoffe im KfK gezeigt.

\section{Das System KADABRA}

\subsection{Die Aufgabe}

Dieses System (Karlsruher Datenbank für radioaktive Abfälle) dient der betrieblichen Unterstützung und Kontrolle und gleichrangig dem Berichtswesen der behördlichen Überwachung.bei der Verarbeitung und Lagerung radioaktiver Reststoffe und Abfälle in der Hauptabteilung Dekontaminationsbetriebe (HDB) im KfK.

Die HDB verarbeitet radioaktive Reststoffe aus der KfK und von externen Anbietern (Wiederaufarbeitungsanlage Karlsruhe, Medizin, Industrie...). Die Reststoffe werden je nach Art in einem Verfahren oder nacheinander in mehreren Verfahrensschritten zu endlagerfähigen Abfallgebinden verarbeitet. Die erzeugten Gebinde werden bis zum Abtransport in ein Endlager in der HDB zwischengelagert. Daneben reinigt die HDB kontaminierte Anlagenteile, die nach Behandlung an den Abgeber zurückgehen. 


\section{Zur Größenordnung:}

In der HDB stehen derzeit ca. 15.000 Reststoff-Einheiten zur Verarbeitung an. Jährlich werden ca. 12.000 verarbeitet, dabei entstehen ca. 6.000 Endabfallgebinde. In den Zwischenlagern liegen derzeit ca. 32.000 Endabfallgebinde.

Die HDB besteht aus einer Anzahl von Anlagen, die untereinander und mit externen Stellen Stoffe austauschen und verschiedenartige Ver- oder Bearbeitungsmaßnahmen an diesen Stoffen vornehmen. Solche Anlagen sind z. B.:

$$
\begin{aligned}
& \text { Anlage A - LAW-Eindampfung } \\
& \text { Anlage B - MAW-Eindampfung } \\
& \text { Anlage Z - Zementierung } 1 \\
& \text { Anlage C - Zementierung } 2 \\
& \text { Anlage K - Kerosinreinigung } \\
& \text { Anlage VL - Lösemittelverbrennung } \\
& \text { Anlage VF - Feststoffverbrennung } \\
& \text { Anlage G - Gerätedekontamination } \\
& \text { Anlage S - LAW-Verschrottung } \\
& \text { Anlage L - LAW/MAW-Lager }
\end{aligned}
$$

Die Aufgaben und Verarbeitungsmethoden der verschiedenen HDB-Anlagen sind sehr unterschiedlich:

Anlage A nimmt beispielsweise leicht radioaktive Wässer an und trennt diese durch Verdampfen in nichtaktives Destillat und höheraktives Konzentrat. Das entstandene Destillat wird aus der HDB abgegeben, das Konzentrat von einer anderen HDB-Anlage weiterverarbeitet.

Anlage $G$ nimmt kontaminierte Anlagenteile an, dekontaminiert was davon wiederverwendbar ist und gibt nicht wiederverwendbare Teile zur Verschrottung (Anlage S) ab oder verarbeitet sie direkt zu endlagerfähigen Gebinden.

Die Anlagen der HDB sind untereinander durch ein sehr komplexes Stoffflußnetz verbunden, dessen Schema in Abb. 2 vereinfacht dargestellt ist.

Die Systemanalyse für das System KADABRA zeigt, daß die Anforderungen, die aus den Zielen der behördlichen Überwachung resultieren, zumindest was die zu erstellende und auszuwertende Datenbasis betrifft, so umfangreich sind, daß die betrieblich motivierten Anforderungen nur eine geringfügige Erweiterung darstellen.

Die betriebliche Komponente bereitet Informationen auf

- für die Leitung der HDB. Zur Verfolgung der langfristigen Entwicklung der Betriebsaufaben der HDB und als Entscheidungshilfe zur Anpassung der Verarbeitungskapazität an künftige Erfordernisse werden Informationen benötigt, z. B. über 
- die Entwicklung der Reststoffeingänge, der Reststoffbestände, der Verarbeitung über mehrere Jahre unter Angabe von Reststoffart, verarbeitender Anlage, Abgeber, Mengen, Aktivitäten,

- den Einsatz von Kondititonierungsverfahren,

- die Entwicklung der Bestände an endlagerfähigen Abfällen unter Angabe von Gebindeart, Aktivitäten, Mengen, Abfallerzeuger,

- Kostenabrechnung etc.

- für die Betriebe der HDB. Zur Verfolgung und Steuerung der Arbeiten in den Anlagen und des Materialflusses sowie der Abwicklung von Aufträgen externer Auftraggeber werden Informationen benötigt, z. B. über

- Reststoffeingänge

- Reststoffbestände

- Verarbeitung

- Zwischenprodukte

- Bestände an endlagerfähigen Abfällen

- Behälter-, Chargen-, Anlageninventare

- Abgeber und Aufträge

\subsection{Das Modell}

Um für das umfangreiche Aufgabenspektrum und das komplexe Stoffflußschema (Abb. 2) die Struktur eines Informationssystems zu entwickeln, das den o. g. Anforderungen bez. Flexibilität, Erweiterbarkeit und Anpaßbarkeit genügt, muß zunächst eine modellmäßige Beschreibung der HDB entwickelt werden. Der wichtigste Gesichtspunkt hierbei ist ein möglichst einfaches und einheitliches Modell zu finden.

Zu diesem Modell gelangt man prinzipiell über dieselben Schritte wie bei jeder Erstellung eines Modells. Als erstes sind auf der Basis der Systemanalyse die Charakteristika der Anlage(n) zu selektieren, die durch das Modell wiedergegeben werden müssen. Im folgenden ist darauf zu achten, daß bei allen Schritten in der Modellentwicklung die Abbildung dieser Charakteristika gesichert ist und nicht verloren geht. Das Vorgehen bei der Modellentwicklung beruht dann darauf, daß man versucht, zuerst das Gesamtmodell in Teilmodelle zu splitten - möglichst in mehreren Stufen bis man zu einfachen Bausteinen gelangt, aus denen das Gesamtmodell wieder zusammengesetzt werden kann.

Im vorliegenden Fall gelingt dies bei dem aus den Anforderungen an das Überwachungssystem abgeleiteten Charakteristikaprofil sehr gut. Die Lösung zeichnet sich dadurch aus, daß

a) sich sehr einfache Bausteine definieren lassen und

b) nur eine ganz beschänkte Anzahl von Bausteintypen als Teilmodelle ausreicht, um das Gesamtsystem zusammenzubauen. 
Der erste Zerlegungsschritt ist aus dem schon gezeigten Übersichtbild (Abb. 2) der Materialströme in der HDB direkt ablesbar und teilt die Gesamt-HDB in ihre Anlagen. Der Einzelbaustein 'Anlage' wird als Einheit (schwarzer Kasten) definiert, der mit der restlichen HDB-Umwelt über die Stoffströme verbunden ist, wobei jeder Stoffstrom einem der vier Typen:
1. Reststoff (R)
2. Zwischenprodukt (Z)
3. Endprodukt (E)
4. Material und Zusätze (M)

zugeordnet werden kann.

Dabei gilt:

\section{- Reststoffe (R)}

sind alle zu be- oder verarbeitenden radioaktiven Stoffe, die von der HDB angenommen werden.

Beispiele: Aktive Wässer, brennbare Feststoffe, kontaminierte Anlagenteile

- Endprodukte (E)

sind endlagerfähige Gebinde.

Beispiel: $2001 \mathrm{Faß}$ in Schwerbetonabschirmung

- Zwischenprodukte (Z)

sind Stoffe, die aus einem Verarbeitungsprozeß der HDB entstehen und entweder weiterverarbeitet werden oder wiederverwendbar sind.

Beispiele: Konzentrat, Asche, gereinigtes Gerät

- Material und Zusätze(M)

sind nichtaktive Stoffe, die zur Be- oder Verarbeitung von radioaktiven Stoffen benötigt werden.

Beispiele: Zement, Fässer, Natronlauge

Eine Anlage kann über je einen Eingang für $R, Z, E$ und $M$ und einen Ausgang für $R, Z$ und $E$ verfügen. Eine reale Anlage wird in der Regel nicht alle der möglichen Ein- und Ausgänge tatsächlich benutzen.

Die Einführung der 4 Stofftypen ermöglicht es, die Gesamt-HDB nach Bausteinprinzip aufzubaven (Beispiel Abb. 3).

Man hat lediglich die Anlagen zu definieren und die sie verbindenden Stoffströme jedes Typs festzulegen. Dieses Prinzip erlaubt es, das Modell und damit das KADABRA-System an strukturelle Änderungen anzupassen, z. B. neue Anlagen zu integrieren oder nicht mehr produzierende Anlagen aus dem System herauszunehmen. Es läßt aber auch eine schrittweise Inbetriebnahme des KADABRA-Systems zu, in dem man ausgehend von einer Anlage schrittweise die Folgenden definiert und integriert. 


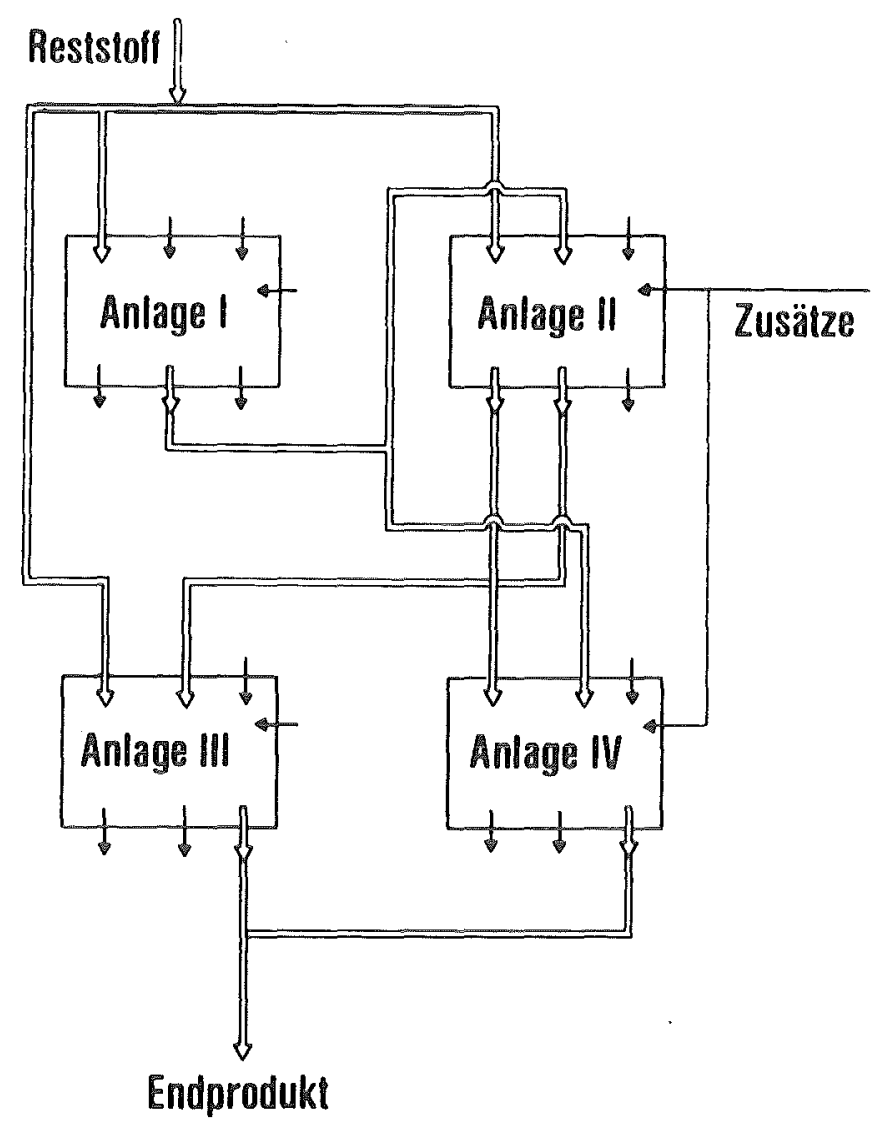

Abb. 3: Modellierung der HDB

Natürlich reicht der bis hierher erreichte Detaillierungsgrad noch nicht für die Beschreibung der Anlage unter den geforderten Aspekten aus.

Die Anlagen, die bis hierher als nicht weiter spezifizierte Kästchen behandelt wurden, von der Aufgabe und Technik her jedoch sehr unterschiedlich sind, müssen genauer betrachtet werden. Eine detaillierte Untersuchung der verschiedenartigen Arbeitsvorgänge in den diversen HDBAnlagen ergibt aber auch hier, daß sich Aufbau und Stoffströme der Anlagen wieder mit einer beschränkten Anzahl von Bausteinen beschreiben lassen, und zwar:

- Lagerposition

Reststoff, Zwischenprodukt oder Endprodukt kann an einer Lagerposition abgelegt und zu einem späteren Zeitpunkt unverändert wieder weggenommen werden.

- Behälter

Der Behälter hat mehrere Eingänge für verschiedenartige flüssige Stoffe, wie Reststoff, Zwischenprodukt und Zusatz oder eine Mischung dieser Stoffe (Behälterinhalt). In einem Behälter sind alle Eingänge homogen gemischt. Durch den einzigen Ausgang des Behälters wird die Mischung als Behälterinhalt entnommen.

- Verarbeitungseinheit

Die Verarbeitungseinheit ist das Modell für alle Komponenten der verschiedenen HDB- 
Anlagen, in denen Stoffe be- oder verarbeitet werden (z.B. Verdampfer, Verbrennungsofen, Zementierung, Presse). Die Verarbeitungseinheit besitzt Eingänge für Reststoff, Zwischenprodukt, Behälterinhalt und Zusätze. Die Zwischen- und Endprodukte, welche die Verarbeitungseinheit verlassen, unterscheiden sich in der Regel in Form und Art vollständig von den Eingangsstoffen.

Jede HDB-Anlage läßt sich aus einer Anzahl dieser drei Bausteine aufbauen. Ein Beispiel zeigt Abb. 4.

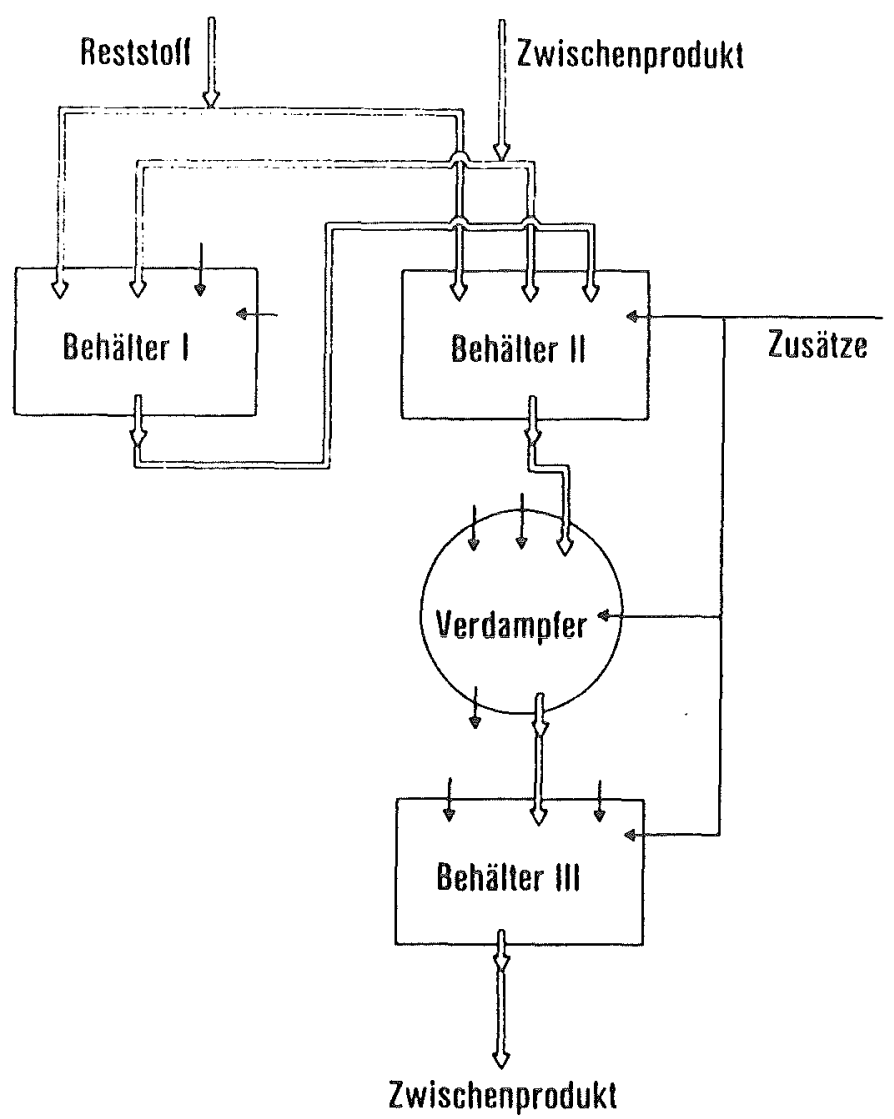

Abb. 4: Modellierung einer HDB-Anlage

Die Anlage verfügt über Eingangsbehälter, in denen flüssige Zwischenprodukte und Reststoffe gemischt werden, über eine Verarbeitungseinheit (Verdampfer) und über einen Behälter zur Aufnahme von Zwischenprodukt. Das Zwischenprodukt wird aus der Anlage abgegeben (in Form von Behälterinhalt).

Auch hier ermöglicht es das Bausteinprinzip, daß das Modell einer Anlage in KADABRA jederzeit änderbar ist. Der Aus- oder Umbau einer HDB-Anlage kann durch Einfügen und Entfernen von Bausteinen im DV-System nachgebildet werden. Ebenso leicht lassen sich die Stoffströme einer Anlage neu definieren. 
Der Nutzen dieses Baustein-Modells besteht nun darin, daß im weiteren Vorgehen nur noch die Begriffe Stoffströme und Anlagenbausteine benutzt werden.Für jeden Typ Stoffstrom und Typ Anlagenbaustein wird ein Datensatz definiert, der den Typ vollständig beschreibt. Jedem realen Stoffstrom oder Anlagenbaustein entspricht dann im System ein Datensatz. Da die Zahl der Anlagenbausteintypen beschränkt ist, ist auch die Anzahl möglicher Funktionen, die mit den Stoffen in oder zwischen den Anlagenbausteinen ablaufen können, beschränkt, z. B.:

- Transfer von einem Behälter in einen anderen,

- Transfer aus einem Behälter in eine Verarbeitungseinheit,

- Entnahme eines Zwischenprodukts aus einer Verarbeitungseinheit

Jeder dieser Funktionen hat die Generierung oder Modifikation eines oder mehrerer Datensätze zur Folge. Zu jeder Funktion wird es daher im DV-System einen Modul geben, der die zu dieser Funktion gehörenden Daten erfaßt und die Modifikationen in den Datensätzen durchführt. Das Vorgehen beim Entwurf hat zur Folge, daß diese Module anlagenunabhängig und in allen Anlagen anwendbar sind, in denen der Stofftyp, der Anlagenbaustein und die Funktion auftritt. Dabei ist jedoch die Benutzeroberfläche der Dialoge jeweils anlagenspezifisch parametriert, sodaß der Anwender sich in seiner speziellen Umwelt bewegt.

Ganz entsprechend sind Berichte z. B. über aktuellen Bestand, chemische Zusammensetzung oder Bilanzen entweder einem Stoffstrom oder einem Baustein zugeordnet und damit anlagenunabhängig und mehrfach verwendbar. Daneben gibt es allerdings auch Berichte, die nur einer Anlage oder der Gesamt-HDB zugeordnet sind.

Wie aus der Struktur des Bausteinsystems ersichtlich ist (Abb. 5 und 6), läßt es eine Materialverfolgung in beide Richtungen des Materialflusses zu.

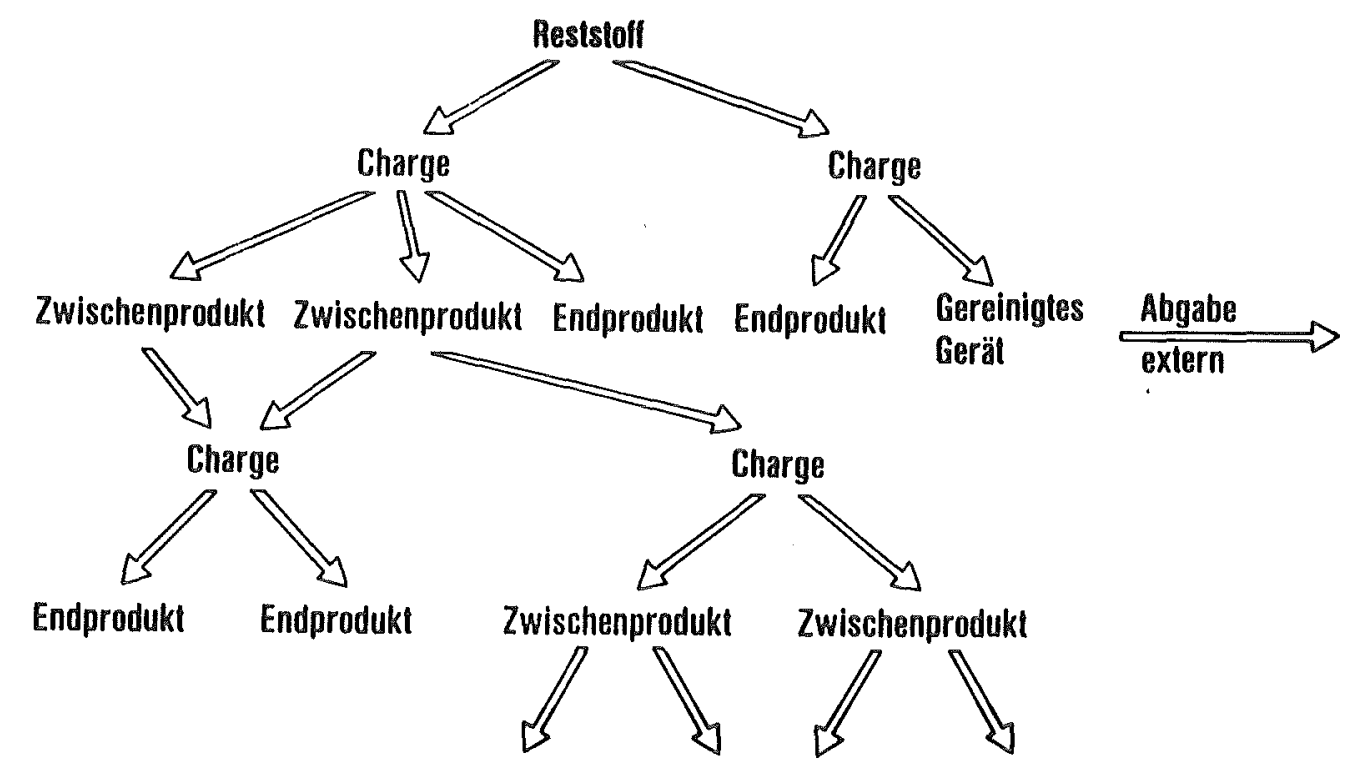

Abb. 5: Verbleib von Reststoff 


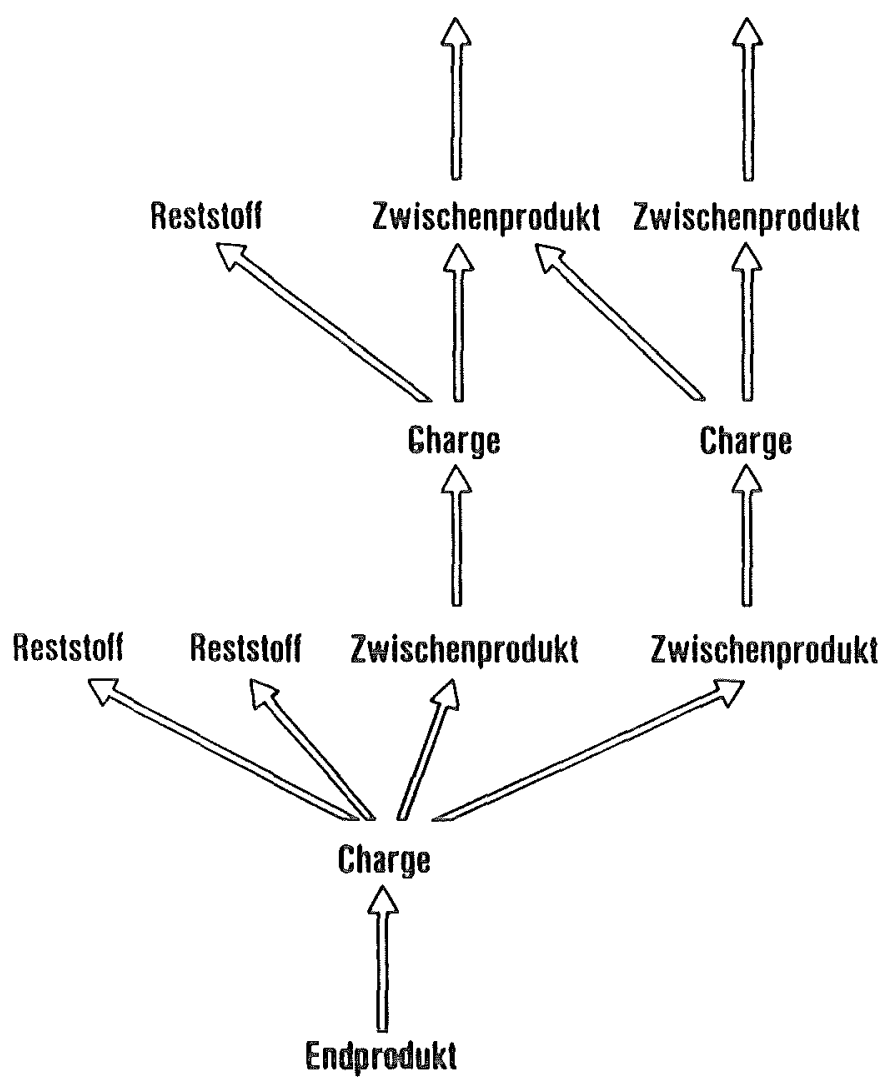

Abb. 6: Rückverfolgung

Die von KADABRA gespeicherten Daten ermöglichen es, den Weg des Reststoffs ausgehend von den Reststoffdaten über die Verarbeitungsschritte (Chargen) und Zwischenprodukte bis zu den Endprodukten zu verfolgen.

Ebenso kann umgekehrt der Pfad vom Endprodukt über die Chargen und Zwischenprodukte zu den Reststoffen zurückverfolgt werden. So kann jederzeit Auskunft über den Verbleib von radioaktiven Stoffen, über die aktuelle Verteilung und die Verursacher von radioaktiven Abfällen gegeben werden.

Das Materialflußnetz gibt weiter die Möglichkeit, die für die Überwachung nötigen Bilanzen auf beliebigen Ebenen aufzustellen, z. B.:

- für jeden Knoten (Verarbeitungseinheit, Behälter.....)

- für eine bel. Aggregation von Knoten (Anlagenbereich, Anlage, Gesamt-HDB). 


\title{
3.3 Die Realisierung
}

Zur Realisierung von KADABRA folgendes:

Es wird ein IBM-Rechner des Typs 4331 mit 2MB Hauptspeicher eingesetzt. Es stehen 2 Plattengeräte 3370 mit je 570 MB zur Speicherung der Datenbank zur Verfügung.

Zum Zweck der Datenerfassung mittels Dialogen und zur Beantwortung von Anfragen bzw. Berichtserstellung kann über 16 Bildschirmeinheiten und 6 Druckeinheiten, die in den Warten der Betriebe und im zentralen Datenbüro der HDB installiert sind, mit dem System kommuniziert werden. Weiterhin ist dieser Rechner mit den Großrechenanlagen in der KfK gekoppelt.

Zur Implementierung des Programmsystems wird als Standardsoftware das Datenbanksystem ADABAS eingesetzt, die Programmierung erfolgt weitestgehend mittels der ADABAS Datenbanksprache NATURAL. Das System ist seit Januar 1985 in Betrieb und erfüllt die gestellten Aufgaben. Die Akzeptanz beim Betreiber ist gut. Neue Anwenderwünsche führen zu kontinuierlichen Erweiterungen.

\section{Die Übertragbarkeit des Konzepts}

Wesentliches Charakteristikum des Konzepts, das auf dem geschilderten Modell basiert, sind seine

\author{
Anpaßbarkeit \\ Erweiterbarkeit \\ Flexibilität.
}

Auf die Anpassungsfähigkeit gegenüber technischen und organisatorischen Änderungen in der HDB wurde bereits hingewiesen. Das Konzept läßt sich aber auch auf andere HDB-ähnliche Betriebe ausweiten; in letzter Konsequenz zu einem nationalen System, das die radioaktiven $A b-$ fallströme der gesamten BRD verfolgt. Gedanken hierzu waren auch tatsächlich bei Projektbeginn im Gespräch und beeinflußten den Entwurf. Politische Gründe machten aber eine Realisierung eines solchen nationalen Systems undurchführbar, so daß in der Realisierung dieses Ziel nicht weiter verfolgt wurde und das System auf die HDB beschränkt blieb.

Die prinzipiellen Gedanken, die dem Konzept zugrundeliegen, sind eigentlich Basis eines jeden Materialverfolgungssystems, damit nicht eingeschänkt auf die beschriebene Anwendung, sondern sicher auch übertragbar auf die Überwachung der Materialströme in der Verarbeitung und Lagerung von konventionellem Abfall. Dieser Schritt wurde gemacht beim Entwurf eines Überwachungssystems für eine Sondermülldeponie, auf das hier, da das System noch nicht realisiert ist, nur kurz eingegangen werden soll. 
Das System setzt auf ein bereits vorhandenes betriebliches System auf. Es ist als verteiltes on-line System konzipiert; $d$. h. die für die Überwachung der Deponie benötigten Daten werden direkt nach Freigabe vom Deponierechner in das Rechnersystem der Überwachungsbehörde übertragen und dort umfassenden Prüfungen auf Konsistenz, Plausibilität, Zulässigkeit bez. den Genehmigungen etc. unterworfen. Unregelmäßigkeiten werden der Behörde sofort gemeldet.

Die Arbeiten an Systemanalyse und Entwurf zeigten hier, daß ein System, das nur dem Ziel der Überwachung dient, nicht sinnvoll ist. Das System ist deshalb so konzipiert, daß es darüberhinaus Aktivitäten des Genehmigungsverfahrens unterstützt. Weiter zeigte sich während der Arbeiten, daß der im Zuge der Überwachung gesammelte Bestand an Deponiedaten für weitere Funktionen bestens genutzt werden kann, so z. B.

- verbesserte Unterstützung bei der Genehmigung

- Berichte, Statistiken und Recherchen bez. aktuellem Bestand

- betriebliche Disposition und Planung

- Planung neuer Anlagen

Hierbei ergibt sich ein neuer Aspekt der Überwachung, daß nämlich durch die Überwachung die Kenntnis über das überwachte Objekt wächst und damit die Optimierung der Überwachung oder des Objektes selbst möglich wird.

\section{Literaturverzeichnis}

11/ Kerpe, R.; Jaeschke, A.:

Distributed Nuclear Materials Accounting System.

Proc. 7th ESARDA Symposium on Safeguards and Nuclear Material Management, Liège, Belgium, 21-23 May 1985, p. 49-52 (1986).

12/ Weidemann, R.; Polster, F.J.:

KADABRA: Informationssystem für die Abfallbehandlung in der HDB.

5. Statusbericht des Projektes Wiederaufarbeitung und Abfallbehandlung des Kernforschungszentrums Karlsruhe, 20.-21. März 1984, KfK-3740, p. 436-453 (1984).

13/ Polster, F.J.; Weidemann, R.; Stegmaier, W.:

KADABRA.

Kernforschungszentrum Karlsruhe, KfK-3963 (1985). 


\title{
Bystere zur Rernreaktor Ernueberwachung
}

Roneepte und Realisierungen

\author{
A . Rudole \\ Dornier System GrabH \\ Postfach 1360, 7990 Friedrichshafen
}

\section{Systere zur Rernreahtorfernueberachung}

honzepte und Realisierungen

\section{Zusammentassung}

In diesem Beitrag werden allgemeine konzepte und Beispiele realisierter Rernreaktorfernueberwachungssysteme (Krues) vorgestellt. Einleitend sind einige der Aufgaben eines RFUEs aufgefuehrt. Drei konzepte fuer den Aufbau von RFuEs werden in dem folgenden Abschnitt aufgefuehrt und allgemein bewertet. Aus einer Anzahl realisierter systeme werden anschliessend drei RFUEs beispielhaft vorgestellt. Anhand des KFUE-Baden-Wuertemberg wird eine etwas detailliertere Darstellung vorgenommen. Abschliessend wird eine kurze Prognose bezueglich der weitere Entwicklung der KruE-Systeme gegeben.

\section{Gliederung}

1. Aufgaben der Kernreaktorfernueberwachung

2. Konzepte

2.1 Systeme mit direkter Messwerterfassung

2.2 systeme mit dezentralen stationsrechnern

2.3 systeme mit dezentraler Architektur

3. Realisierte KFUE-Systeme

3.1 Eigenueberwachungssystem THTR

3.2 KFUE Rheinland-Pfalz

3.3 KFUE Baden-Wuerttemberg

4. Genereller Aufbau eines KFUE-Systems am Beispiel des KFUE-Baden-Wuerttemberg

4.1 Funktionaler Aufbau

4.2 Software-struktur

4.3 Massnahmen gegen Datenverlust und -verfaelschung

5. Perspektiven der zukuenftigen Entwicklung 
Syctere sur Rerncektorternueberwachung

koneepte und keallsierungen

\section{Aurgaben der Rernreaktor fernueberwachung}

Die systeme zur Rernreaktorfernueberwachung werden von den zustaendigen Aufsichtsbehoerden im wesentlichen fuer folgende Aurgaben benoetigt:

- Unterstuetzung der zustaendigen Behoerde bei der Ueberwachung der Rernkraftwerke.

- Rontinuierliche Erassung, Verarbeitung, Speicherung. Uebertragung, Darstellung und Dokumentation von Messwerten.

- Durch die staendige Erfassung von geeigneten Messgroessen sollen die mit dem Normalbetrieb verbundenen Emissionen und Imnissionen ueberwacht werden.

- Fruehzeitiges Erkennen von betrieblichen Unregelmaessigkeiten.

- Ueberpruefen der Daten auf vorgegebene Grenzwerte. Ueberschreitungen koennen zu Alarmen fuehren, die dann eine autom. Alarmierung der Aufsichtsbehoerde ausloesen.

- Durch fuehren von Ausbreitungsrechnungen, um die radiol. Belastung in der Umgebung eines Rernkraftwerks abschaetzen zu koennen. 
Eyetere sur Gernreator Cernueberwachung ronepte und rearrierungen

\section{2. ronzepte}

cin rernreaktorernueberwachungssysten laesst sich in rolgende Romponenten auteilen. Es besteht aus einen oder aehreren KKW-Systemen und jedes KKW-System gliedert sich wiederum in unterkomponenten. Abbildung 1 enthaelt eine prinzigielle Darstellung ines rkW-Systems aus RuE-sicht. Dementsprechend es in tolgende allgemeine komponenten gegliedert werden.

- Messgeraete in ramin

- Messgeraete in der Anlage

- Meteorologische Messgeraete

- Imissions-Messgeraete

1. Noverate in Kanin

2. Mogerthe in Aer Ange

3. Meteonologische MeBoerite

4. Imonissions-Pleboerïte

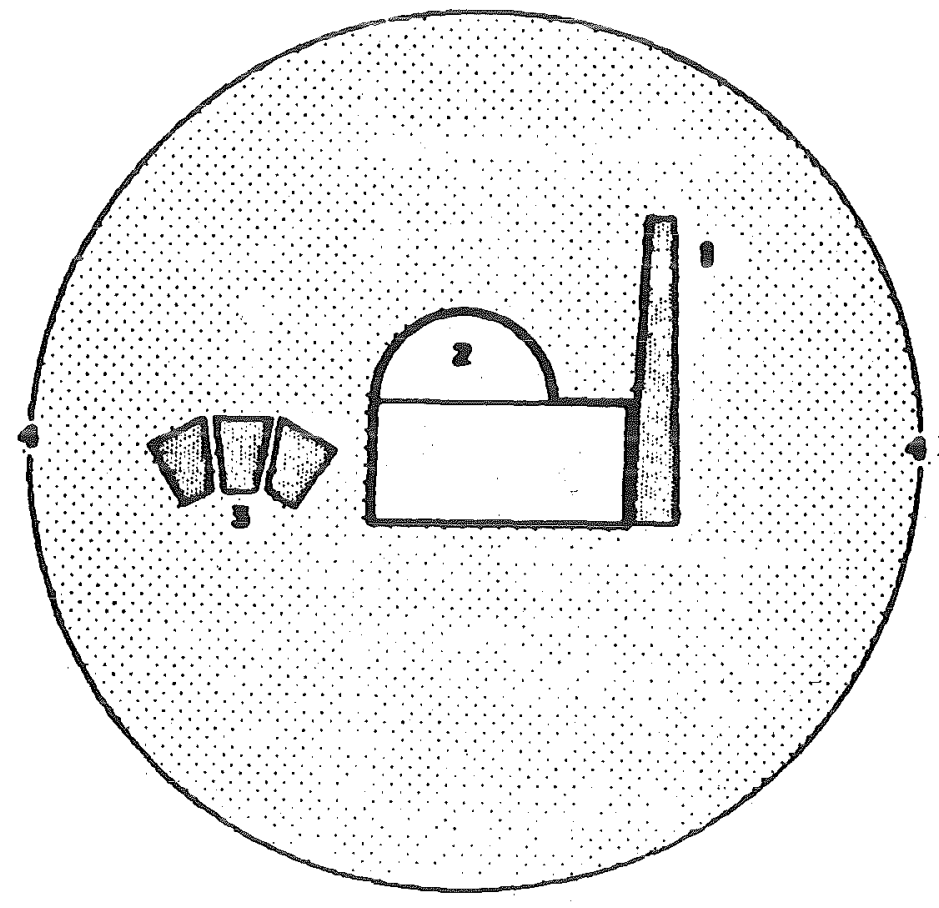

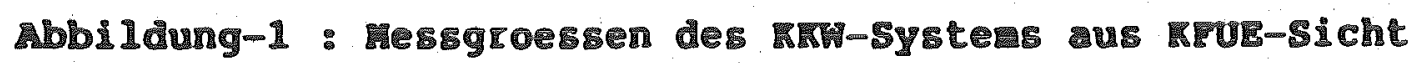

Fuer die Erfassung der Hessgroessen aus den einzelnen Rrw-Systemen koennen verschieden Konzepte bzw. Hessnetzarchitekturen verwendet werden. Diese verschiedenen Konzepte solIen in den Folgenden Kapitein aurgezeigt werden. 


\section{Bystee zur Rernieaktor fernueberwachung}

ronzepte und Realisierungen

\section{1 systeme mit direkter hesswerterissung}

systeme mit direkter Messwerterassung sind nur cuer messnetze mit relativ geringer hessgeraeteanzahl und geringer oertlicher Ausdehnung geeignet. Im Rahmen der leistungsiaehiger werdenen Kleinrechner inden solche Architekturen heute kaum noch verwendung.

Die Vorzuege von systemen mit direkter Messwerterfassung bestehen in ihrer kompakten und gut ueberschaubaren Ronfiguration. Desweiteren sind relativ geringe Hardwarekosten anzusetzen.

Aus Redundanzgruenden ist es jedoch nachteilig, dass alle Systemfunktionen wie

- Datenerfassung.

- Datenverarbeitung.

- Informationsaufbereitung und -ausgabe,

- etc.

vom Zentralrechner durchgefuehrt werden muessen.

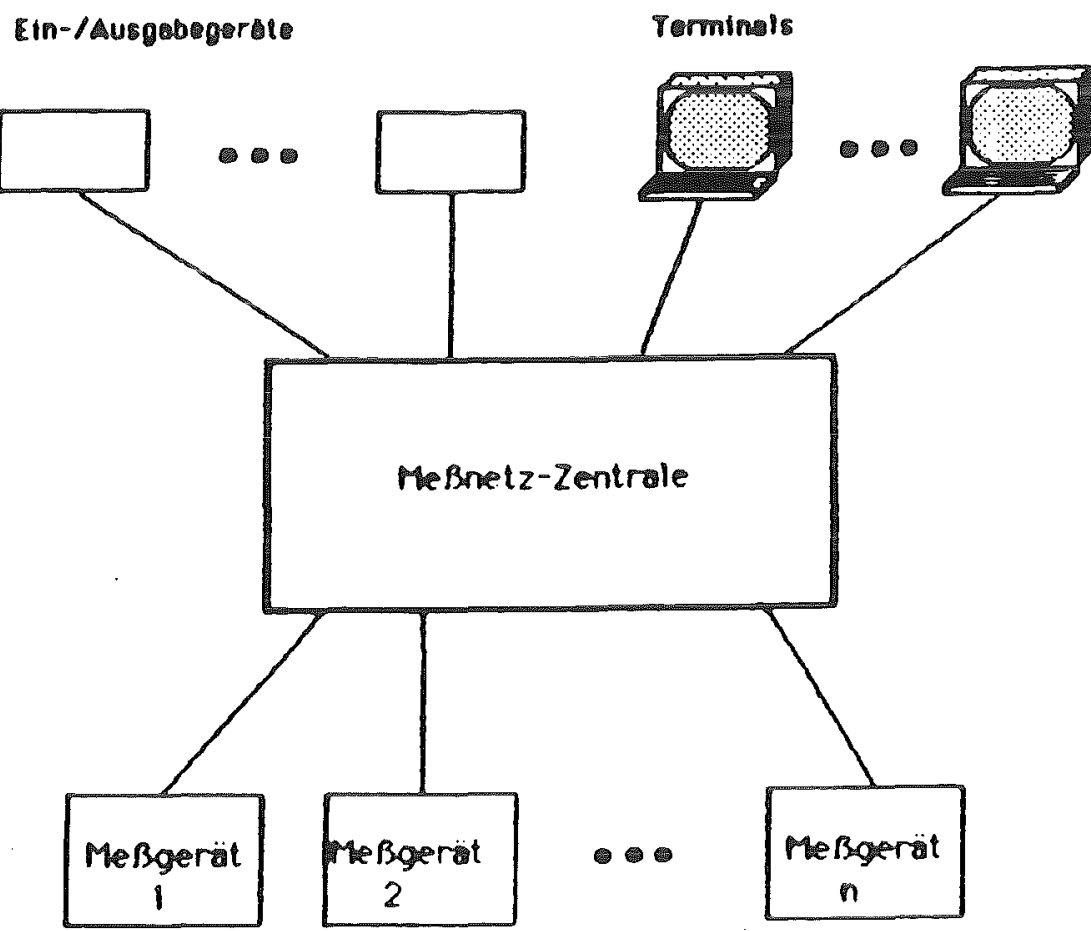

Abbildung-2: System it direkter hesswerterfassung 


\section{Systere zur Rernceaktor fernueberwachung} ronzepte und Resilsierungen

\section{2 systeme mit dezentralen stationsrechnern}

Ronzepte mit dezentralen stationsrechnern, finden einen weiten Einsatzbereich, wie z.B. Eigenueberwachungssysteme der Rraftwerke oder RFUEs auf Landesebene.

Mit diesem Ronzept ist der Aurbau einer klaren und uebersichtlichen systemstruktur moeglich. Die Redundanz in der Erfassung ist gegenueber dem konzept mit direkter Messwerterfassung im Bereich der Datenerfassung verbessert. Da diese jetzt auf meherere Rechner verteilt ist, und damit eine Parallelverarbeitung stattindet, koennen relativ viele Messgroessen erfasst werden.

Die Verbesserung der Redundanz und Flexibilitaet wird erkauft mit mehr Hardwarekomponenten und mit einer komplexeren Softwarestruktur. Diese ist unter anderem durch die Kopplung von zentral- und stationsrechner zu erklaeren.

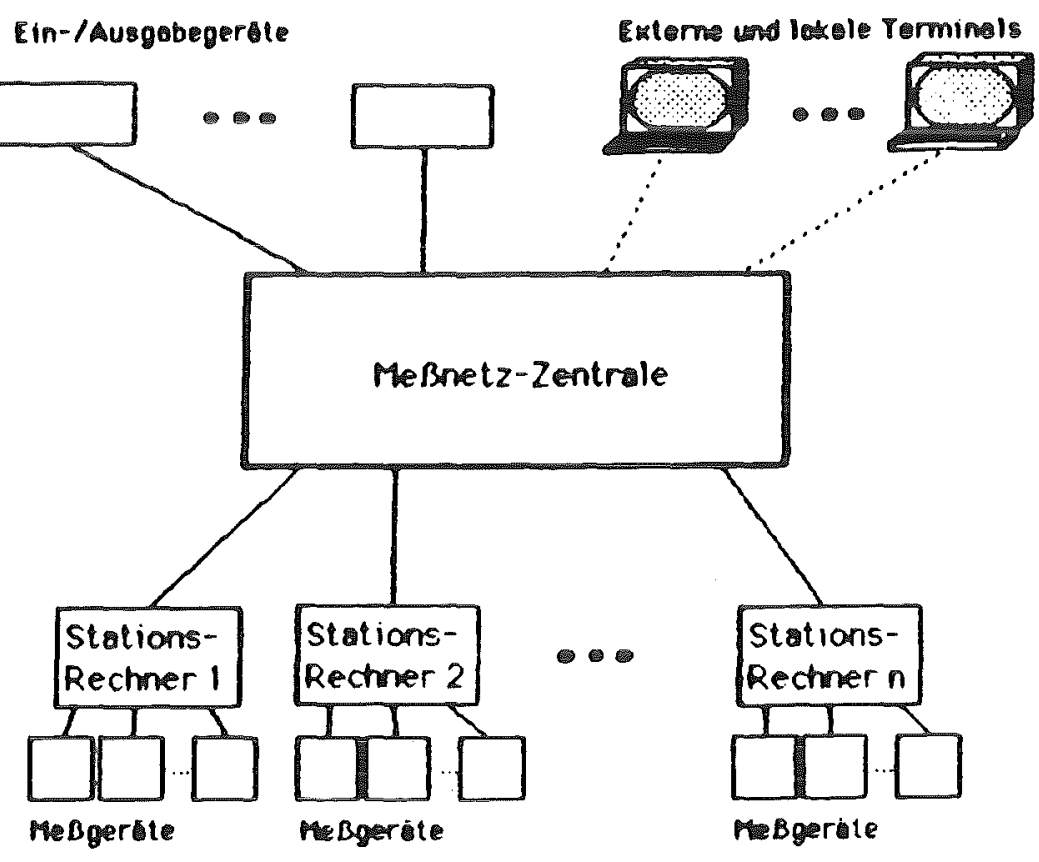

Abbildung-3: Syster it dezentralen stations-Rechnern 
systere cur Rernceaktor ernueberwachung ronzepte und Reallorerungen

\section{3 systeme nit dezentraler Architektur}

Der Einsatzbereich von messnetzen mit dezentraler Architektur ist das gesante spektrum der Messnetze baw. RFues.

Die Vorzuege einer solchen Architektur duerten in der grossen Redundanz und der moeglichkeit der autarken, anwenderspezifischen Datenaufbereitung liegen.

Die Nachteile fuer ein solches system sind:

- Die einzelnen stationsrechner muessen relativ aurwendig und leistungsstark sein.

- Durch den allgemeinen Datenzugriff ist die Qualitaet (Rorrektheit,allg. Verstaendlichkeit) der Daten nur schwierig zu gewaehrleisten.

- Fuer ein solches system ist ein aufwendiges Netzwerkkonzept erforderlich dessen hauptproblem in der konsistenz der Daten zu suchen ist. Solch in, ueber viele stationsrechner verteiltes Datenbanksystem ist bisher in der Praxis noch nicht verfuegbar. Zukuentig sind solche systeme allerdings zu erwarten.

- Im Alarmfall ist damit zu rechnen, dass viele Anwender die Daten einer bestimnten station benoetigen, und diese Einzelstation dann zum 'Flaschenhals' des systems wird. Der Vorteil eines verteilten systems ginge in diesem Fall verloren.

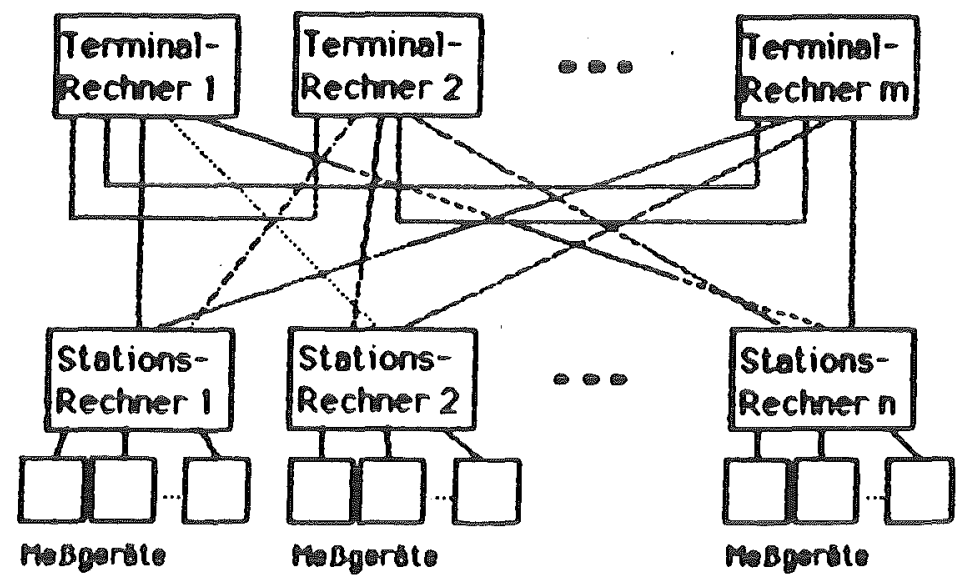

Abbildung- Deqentrales Messnetzsyster 
Systere zur Rernreaktoriernueberwachung

ronepte und Rerigierungen

\section{Realisierte KrUe-Systere}

\subsection{Eigenueberwachungssystem THTR}

Fuer das Eigenueberwachungssystem THTR-300 wurde eine hierarchische systemstruktur eingesetzt. In der Dacenerfassung wurden abgesetzte signalrechner (Dornier) verwendet, die an den zentralrechner vorgepruefte und vorgerechnete Daten lieEern. An den Erfassungsrechner fuer die Meteorologiedaten ist ein Sodarsystem fuer die Erfassung hoehenspez. meteorologischer Daten des standorts angeschlossen.

Der zentralrechner nimmt vor allem Aufgaben war, wie

- Datenuebertragung von signalrechner zun zentralrechner

- Grenzwertueberpruefung und Alarmausloesung

- Verwalten der Daten

- Bereitstellen einer komfortablen Benutzerober laeche

- steuerung der autom. Funktionen im Messnetz

- usw.

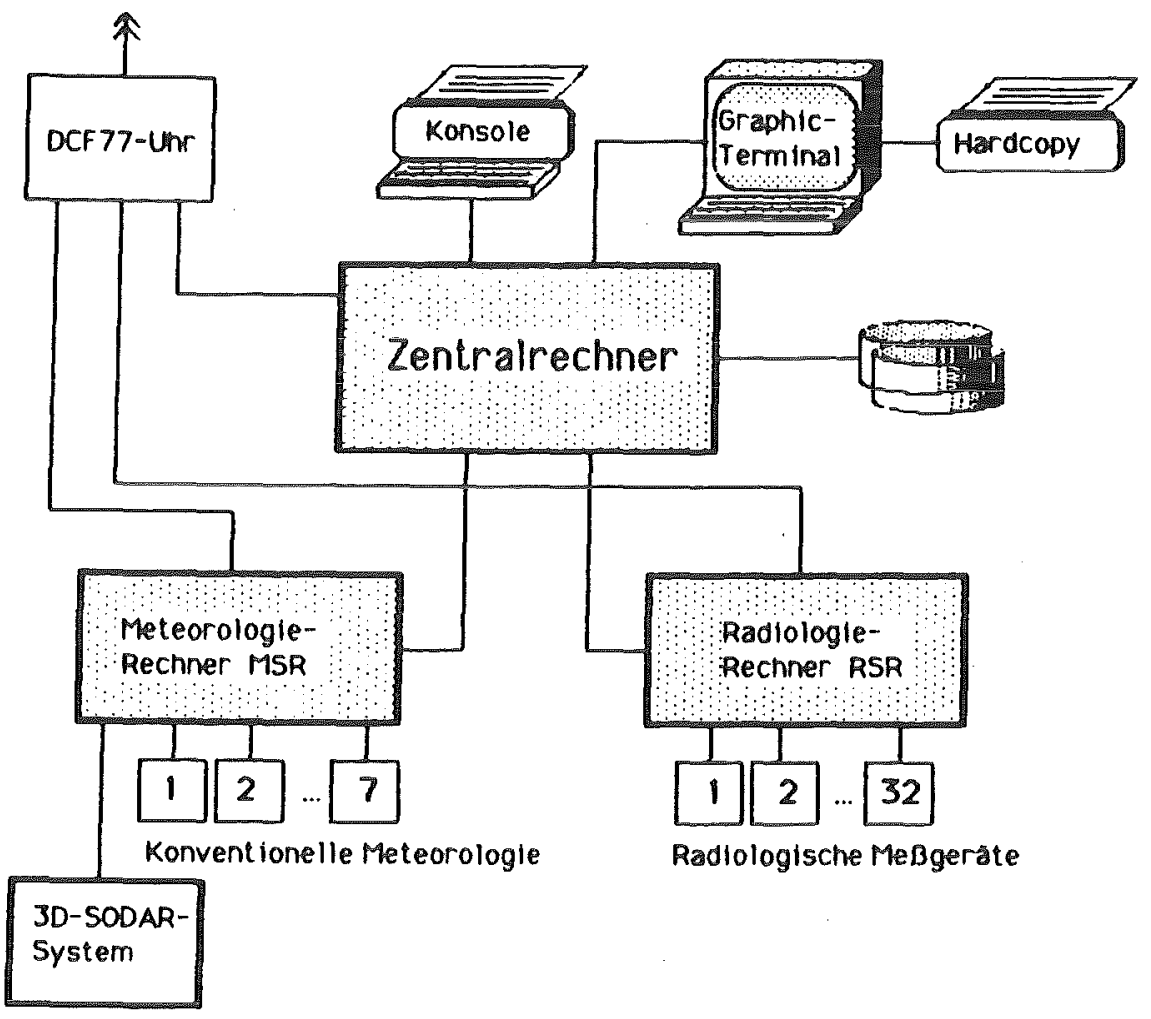

Abbildung-5: Eigenueberwachungssyster TRT-300 


\section{Systere zur Rernreaktorfernueberwachung ronzepte und nealisierungen}

\subsection{KFUE Rheinland-P}

Fuer das RFUE-Rheinland-pralz wrote in der zentrale aus Redundanzgruenden ein Doppelrechnersystem eingesetzt.

Die verwendete Messnetzstruktur ist hierarchisch. Die strenge Hierarchie ist jedoch bei den Verbindungswegen $z u$ den sogenannten EKS (Einkomponentenstationen) durchbrochen, um hier die Redundanz zu erhoehen. Fuer die Datenuebertragung von den Datenerfassungsstationen zur zentrale murde das Datex-L-Netz eingesetzt. Die MKS-Stationen sind ueber Datex-L-2400 und die EKS-Stationen ueber Datex-L-300 an die zentrale angebunden.

Die KrUE-Anwender sind sowohl direkt als auch ueber Datex-L- Leitungen an das KFUE-System angeschlossen, wobei fuer beide Gruppen der Funktionsumfang gleich ist. Ausgenommen hiervon sind spezielle systemfunktionen.

Die Datenhaltung in der Zentrale erfolgt auf beiden Rechnern, die mittels DECNET miteinander verbunden sind. Die Rechner in der Zentrale nehmen bezueglich der Benutzeroberflaeche die gleichen Aufgaben war, wie der Zentralrechner im THTR-System.

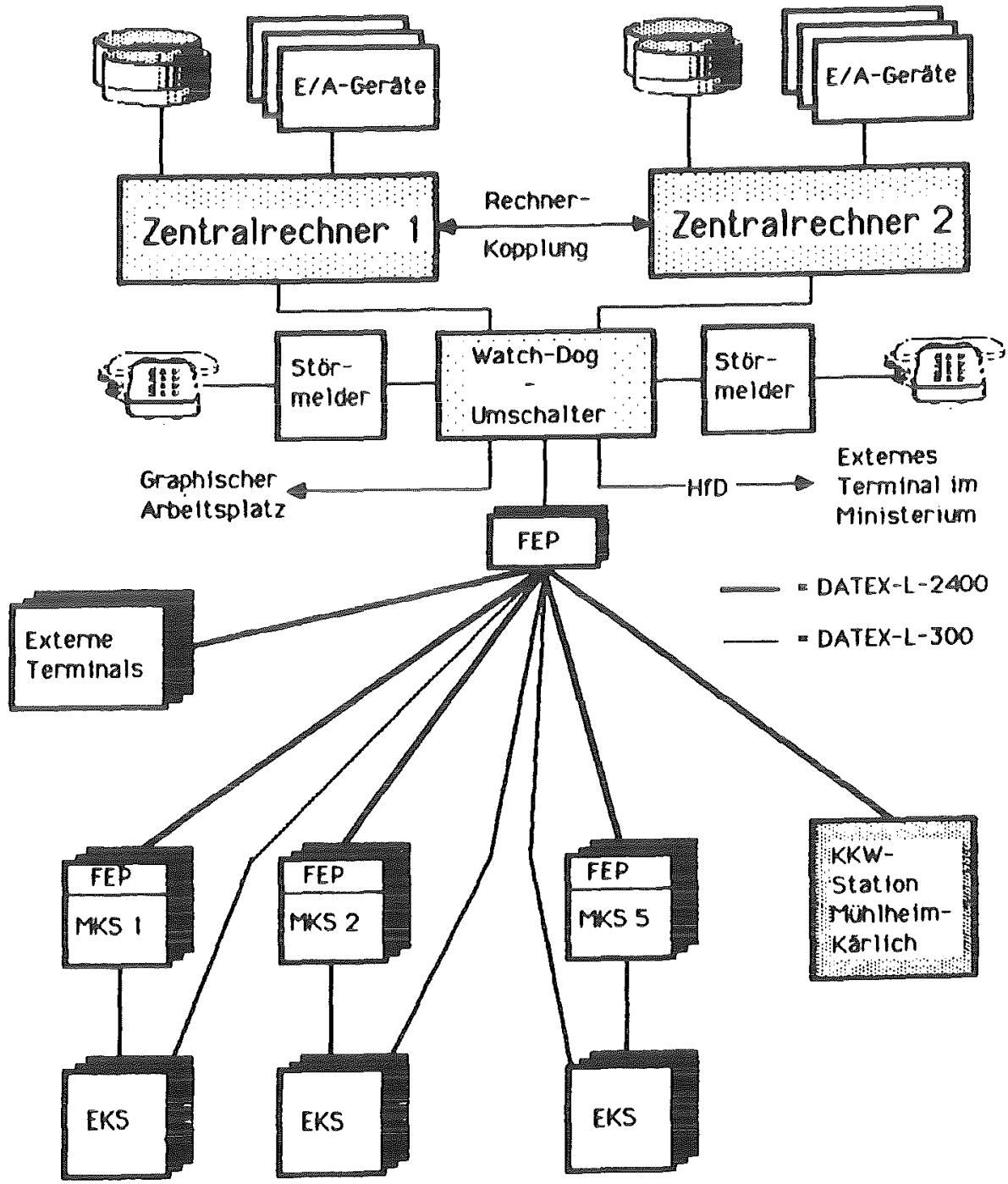


Bystere gur rernreaktor Ternueberwachung Rongepte und Realislerungen

\subsection{RFUE Baden-Wuerttemberg}

Fuer das RFuE-Baden-Wuerttemberg ist wie fuer Rheinland-PIalz aus redundanzgruenden ein Doppelrechnersystem in der zentrale eingesetzt worden. Die beiden Rechner werden nit einem watch- Dog ueberwacht, um einen Ausfall rechreitig zu detektieren. Beide Rechner verfuegen ueber stoermelder, die eine Alarmierung der zustaendigen Behoerden ueber Telefon oder Euro-Call ermoeglichen.

Die Datenerfassungsstationen in den einzelnen kratwerken sind ueber das Datex-L-2400-Netz an die zentrale angeschlossen. Die Immissionsmessringe, die um die Rraftwerke liegen, sind ueber das Datex-L-300-Netz angeschlossen.

Die Datenerfassungsstationen in den kKWs sind aus redundanzgruenden in drei Einzelsysteme Unterteilt, deren Informationen von redundant ausgelegten Datenaufbereitungsstationen gesammelt und an die zentrale uebertragen werden.

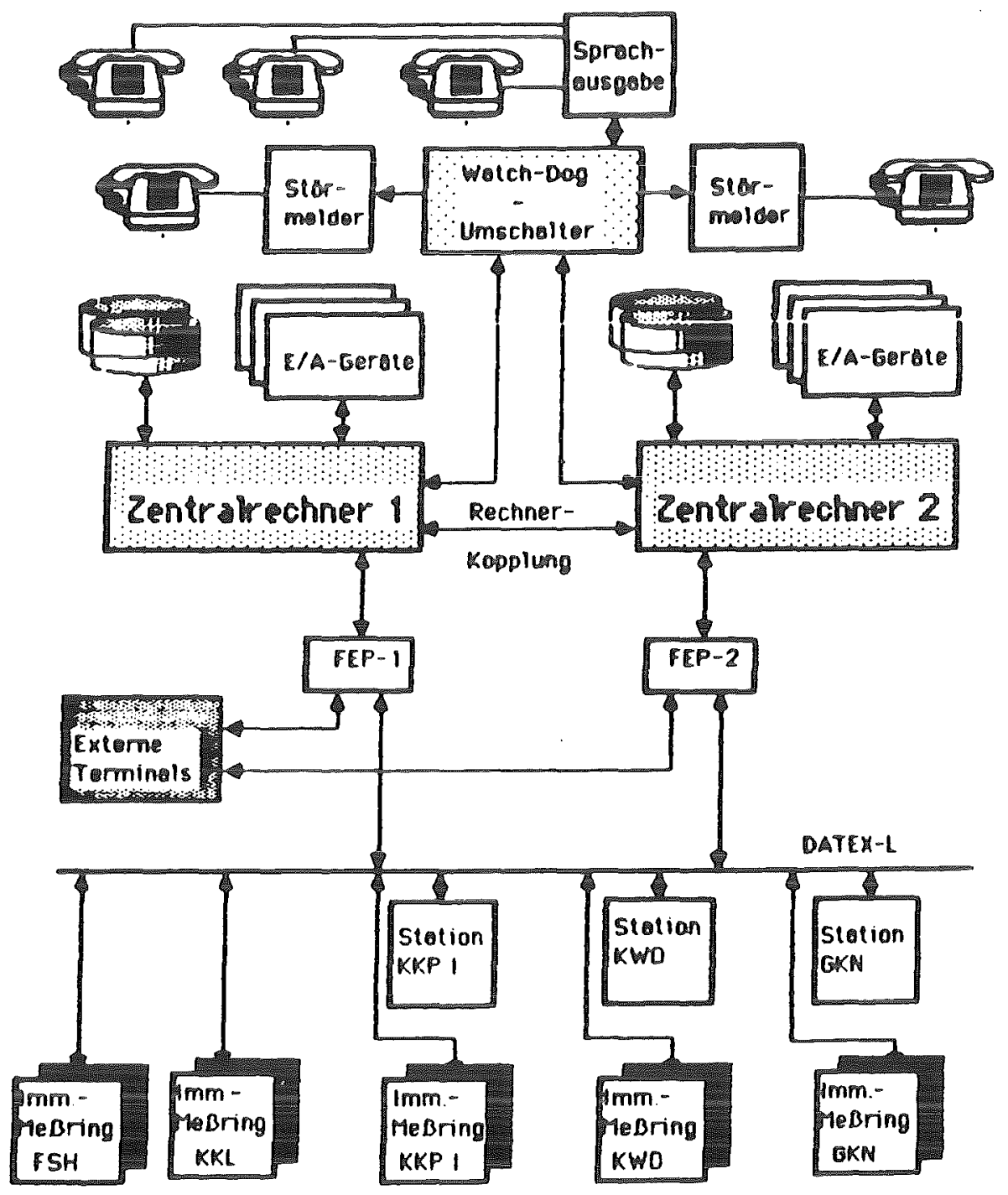

Abbildung-7 : RPUE-Baden-Wuertemberg 


\section{systeae zur hernieaktorfernueberwachung}

monepte und Realis lerungen

Im Gegensatz zum System in Rheinland-PEalz und im THTR werden in den Datenerfassungsstationen nur Rohwerte erzeugt und gespeichert. Diese Rohwerte werden in der zentrale dann zu phys. Werten ungerechnet.

Die Anwender sind sowohl direkt als auch ueber Datex-Leitungen an das KFuE-systen angeschlossen, wobei fuer beide Gruppen der Funktionsumfang gleich ist.

Die Datenhaltung in der zentrale erfolgt auf beiden Rechnern. die ueber DECNET miteinander verbunden sind. Die Rechner

der Zentrale nehmen im uebrigen die gleichen Aurgaben war. wie der zentralrechner im THTR-System, bzw. RFUE-System. 
Systere zur Rernreaktorernueberwachung ronzepte und Realisierungen

\section{Genereller Aufbau eines rrue-Systens Beispiel des RVUE-Baden-buertterberg}

\subsection{Funktionaler Aufbau}

Anhand des KFuE-Baden-wuertemberg wird versucht den Eunktionalen Autbau eines KFUEs darzustellen.

Die Hauptrunktionsbloecke eines KFUEs sind:

- Datener

Erfassung und Vorverarbeitung der messwerte in den Datenerfassungsstationen. Je nach system werden Ereignisse autom. an die zentrale weitergegeben.

- Datenfernuebertragung

Funktionen die die Verbindung zwischen zentrale und den Datenerfassungsstationen sicherstellen. Sie sind fuer den Abrui und Uebertragung der vor ort erfassten Messdaten zustaendig.

- Datenverarbeitung

Die Datenverarbeitung wertet die uebertragenen Daten aus und uebergibt sie an die Dateiverwaltung zur Speicherung. Die einzelnen Groessen werden auf verschiedene kriterien hin ueberprueft. Ueberschreitung von Grenzwerten einzelner Groessen bzw. Rombinationen koennen $z u$ sogenannte radiologische Alarme fuehren, die eine Alarmierung der zustaendigen Behoerde zur Folge hat.

- Ausbreitungsrechnung und Basisdatenerzeugung

Die Ausbreitungsrechnung, die eine Abschaetzung der radiologischen Belastung in der Ungebung eines Rernkraftwerks ermoeglicht, kann automatisch oder manuell angestossen werden. Der autom. Anstoss ist imner die Folge der Ausloesung eines radiologischen Alarms durch die Datenverarbeitung.

- Dateiverwaltung

Die Dateiverwaltung stellt eine hierarchische Datenbasis dar. Die Daten koennen ueber spezielle Aufrufe die bis zu drei Schluessel (KKW-System, Datum, Messgeraet) enthalten koennen ausgelesen bzw. geschrieben werden. Alle Daten, die auf platte geschrieben werden, werden auch ueber die Rechnerkopplung an den zweiten Rechner gesendet. 


\section{Systere zur Rernceaktor ternueberwachung}

\section{Ronzepte und Rearisierungen}

- Rechnerkopplung

Die Rechnerkopplung uebertraegt die Daten zwischen den beiden Rechnern, um einen konsistenten Datenbestand der beiden Rechner zu ermoeglichen.

Die Rechnerkopplung fuehre beim start der Rechner alle Funktionen Euer einen automatischen Update durch.

- Anwender runktionen

Unter Anwenderfunktionen ist die gesamte Benutzeroberflaeche des KFUEs zu verstehen. Hierin sind runktionen zur Darstellung der Messwerte in graph. Form bzw. in Protokollform, sowie Funktionen zur korrektur von Daten usw. enthalten.

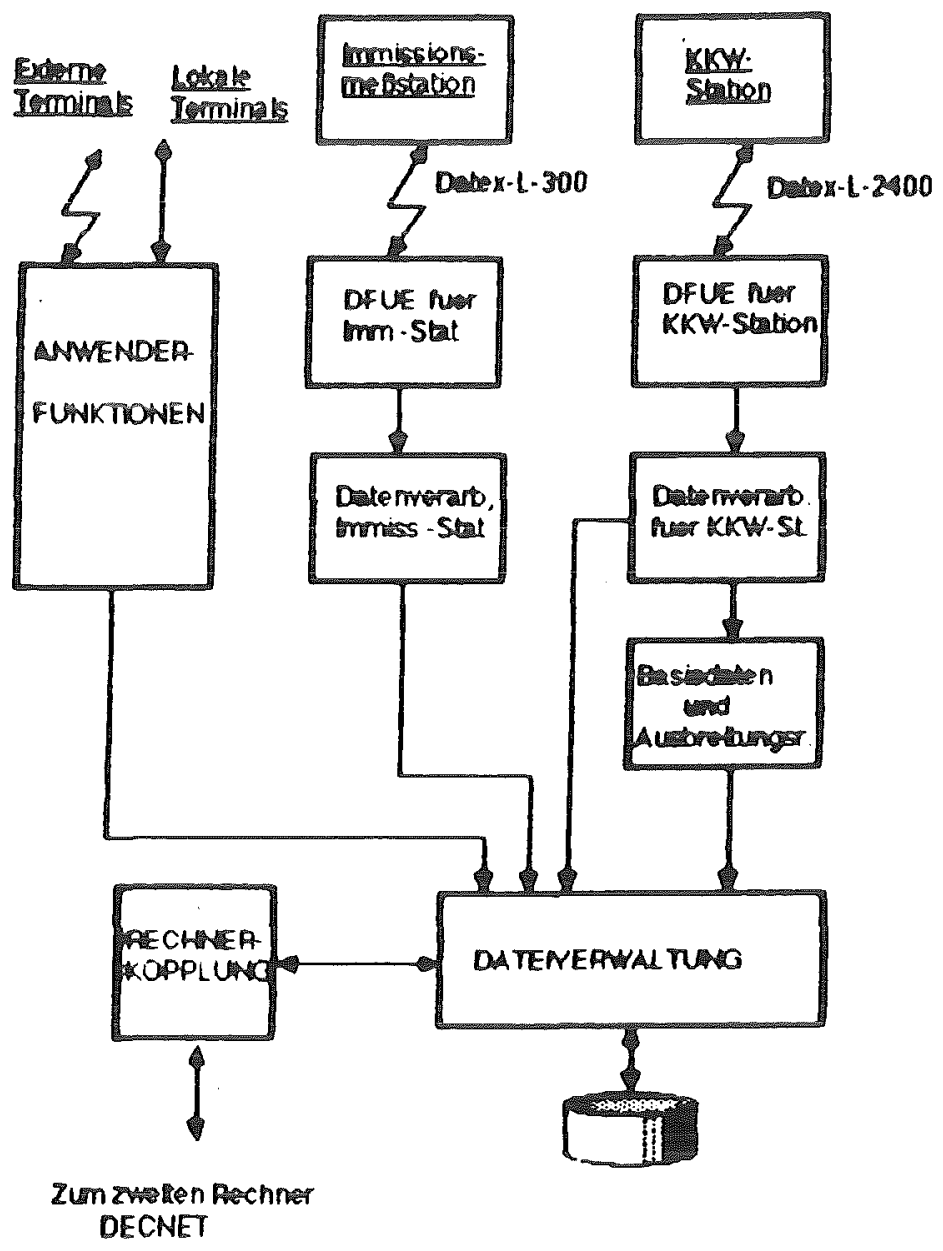

Abbildung-8 : Funktionaler Aufbau 


\section{Systeae zur Rernreaktor fernueberwachung}

rongepte und Rearisierungen

\subsection{Solware-struktur}

Die sortwarestruktur des KFue lehnt sich sehr eng an die cunktionale struktur des systems an. Es gliedert sich in zwei Bloecke. Der eine wird von den Prozessen des on-Line-syster gebildet und der andere von den Prozessen der BenutzeroberHaeche.

DFUE-Verwaltung, DFUE-FEP1 und DFUE-FEP2 sind Datenfernuebertragung- und Datenverarbeitungsprozesse fuer die Daten der Imnissionsmessringe. Sie werden ueber die autom. Ablaufsteuerung aktiviert bzw. zeitlich gesteuert.

Fuer die Daten der Kraftwerkstationen Euehrt die DFUE-DATEX-L2400 die Funktionen der Datenfernueberstragung durch. Die Datenverarbeitung Euer die Werte der KKW-Stationen wird von einem eigenen Prozess durchgefuehrt, der auch fuer die Grenzwertueberpruefung und Alarmierung zustaendig ist.

Die Datenverarbeitung versorgt den Prozess 'Basisdaten und ON-Line-Ausbreitungsrechnung" mit Informationen. Er Fuehrt unter anderem Datenverdichtungen und Vorrechnungen aus, die der nachfolgende On-Line- und off-Line-Ausbreitungsrechnung zur Berechnung der radiologischen Belastung der Umgebung dienen.

Ale systemweiten Daten, die auf Platte gespeichert sind, werden ueber die Dateiverwaltung gefuehrt. Die zugriffe auf die Dateien sind standardisiert.

Die Rechnerkopplung stellt ein Prozesssystem dar, dass mittels DECNET die Uebertragung der erforderlichen Daten zum Parallelrechner vornimut. In der Rechnerkopplung sind alle up-Date-Funktionen fuer den systemstart integriert.

Die Anwenderfunktionen werden in jeweils eigenstaendigen Prozessen durchgefuehrt. Sie sind ueber gemeinsame Datenbereiche und die Dateiverwaltung mit dem On-Line-System verbunden.

\subsection{Massnahmen gegen Datenverlust und -verfaelschung}

Die Vorkehrungen sind verteilt auf die Datenerfassungsstationen und auf die KFUE-Zentrale.

Massnahmen in den Datenerfassungsstationen

Die stationen werden an unterbrechungsfreie stromversorgungen angeschlossen und verfuegen zusaetzlich ueber batteriegepufferte speicher. Diese speziell gepufferten speicher koennen Daten fuer eine zeitbereich von bis zu drei Tagen aufnehmen. 


\section{Bystere zur Rernceaktorernueberwachung}

\section{ronzepte und rearirierungen}

Massnahmen in der zentrale

- Rann eine station aus irgendwelchen Gruenden nicht abgerucen werden, werden die Daren nach wiederherstellung der Verbindung autom. abgerufen.

- Das system arbeitet auf zwei Rechnern mit eigener Plattenperipherie.

- Nach einen Ausfall der Rechnerkopplung werden alle zu uebertragenden Daten markiert und nach wiederherstellung der Ropplung werden die exforderlichen Bereiche autom. aktualisiert.

- Alle manuell veraenderten Daten werden mit einer speziellen statuskennung versehen.

- Es koennen nur Daten der untersten Hierarchiestufe veraendert werden.

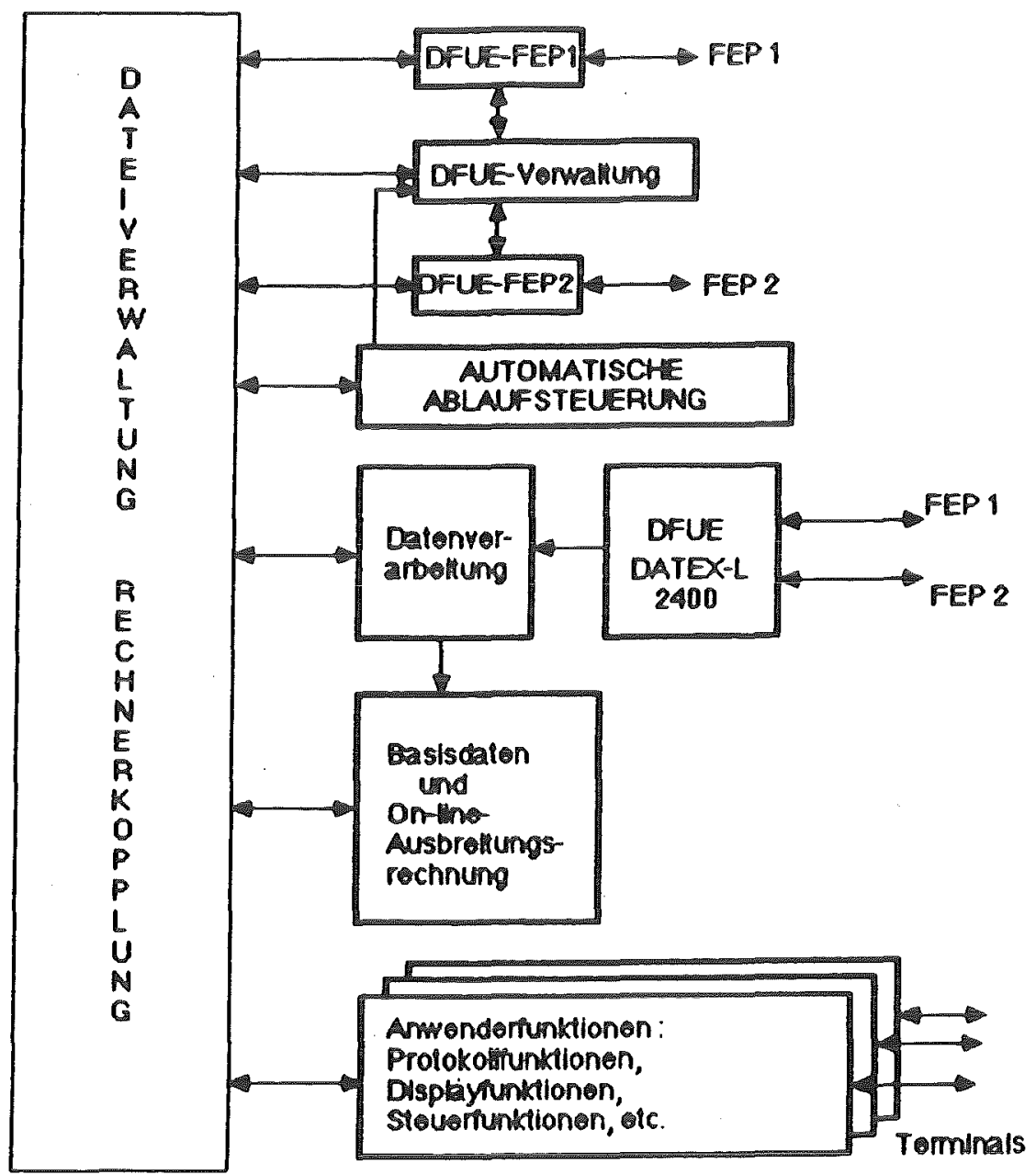

Abbildung-9: Softwarestruktur des KrUE-Baden-Wuertteaberg 


\section{systere gur rernceaktor fernueberwachung}

ronepte und Realis lerungen

\section{Perspektiven der zukuentigen Entwicklung}

In Rahmen der verstaerkten veberwachung der Umwe1t, wird es notwendig werden, dass die Daten verschiedener Umweltmessnetze ueber eine schnittstelle vom Anwender abrufbar sind. D.h. die verschiedenen vorhandenen Messnetze wie RFUEs, Luftmessnetze oder Gewaessermessnetze werden in zukunft aiteinander vernetzt werden muessen. Nur so wird es moeglich sein, die Einzelinformationen schnell und sicher miteinander zu korrellieren und daraus die bestehende Gesantlage zu beurteilen. 

Konzeption und Implementation eines datenbankgestützten Auswertungssystems für LuftmeBdaten

A. Hansen, B. Page, U. Zacharias

Fachbereich Informatik der Universität Hamburg

\section{Zusammenfassung:}

Auf der Basis des Architekturmodels für Methodenbanksysteme wird ein integriertes Auswertungssystem für Luftmeßdaten realisiert. In dem Vorhaben werden eine Datenbankkomponente (SIR), ein statistiksystem als erweiterbare Methodenbankkomponente (SAS) und ein Maskengenerator als Dialogkomponente (IPF) zu einem benutzerfreundlichen Gesamtsystem integriert. Dieses system soll über die mehr kurzfristig orientierten Routineauswertungen in LuftmeBnetzen hinausgehend langfristige statistische Auswertungen mit der Möglichkeit zur Korrelation mit Meteorologiedaten und epidemiologischen Daten sowie eine anspruchsvolle graphische Ausgabe ermöglichen. Es soll gezeigt werden, daB unter Verwendung der Subkomponenten ein mächtiges und leicht erweiterbares system mit vertretbarem Implementationsaufwand erstelit werden kann. In Zusammenarbeit mit der Hamburger Umweltbehorde und dem Luftmeßnetz wurden umfangreiche reale Meßdaten verarbeitet und verschiedene Auswertungen exemplarisch realisiert.

\section{Gliederung:}

1. Einleitung

2. Beschreibung des Auswertunassystems.

2.1. Die Benutzersicht des Auswertungssystems

2.2. Die verwendeten Teilsysteme

2.3. Das Rahmensystem

2.4. Die Datenverwaltung

2.5. Die Statistik

3. AnschluB einer epidemiologischen Datenbank

4. Zusammenfassung und Ausblick

Literatur 
1.

Die elektronische Datenverarbeitung gewinnt in den Umweltbereichen, in denen die Erhebung. Analyse und Verarbeitung umfangreicher Datenmengen eine grobe Rolle spielt, zunehmend an Bedeutung. Im Bereich der Luftüberwachung mit computergesteuerten Mebnetzen werden schon seit längerem Prozeprechner eingesetzt, die wichtige steuerungsfunktionen wahrnehmen, die Erfassung der gemessenen Parameter unterstützen und die vorgeschriebenen standardauswertungen durchführen.

Für die Auswertung und komplexe statistische Analyse vom Massendaten (z.B. Meßwertzeitreihen über mehrere Jahrel sind solche systeme jedoch nicht geeignet. Am Beispiel des Hamburger Luftmennetzes wird dieses deutlich: der dort eingesetzte Prozeprechner ermöglicht es derzeit nur, die Daten für jeweils ein bis zwei Monate zugreifbar zu halten, langfristig werden die Daten auf Magnetbändern archiviert. Die von der TA Luft vorgeschriebenen Auswertungen können zwar mit Hilfe verschiedener Assemblerroutinen durchgeführt werden, sind jedoch nicht flexibel einzusetzen. Exweiterungsmöglichkeiten bestehen nur begrenzt im Rahmen der Möglichkeiten des Prozeßrechners.

Weitergehende Auswertungen und die Verarbeitung umfangreicher Datenmengen erfordern den Einsatz eines größeren Rechners und die Unterstützung durch moderne Softwaresysteme zur Datenverwaltung und -analyse. Ziel dieser Arbeit ist es, mit diesen leistungsfähigen Hilfsmitteln oin system zu konzipieren und exemplarisch zu implementieren, das diese Aufgaben erfüllen kann und darüber hinaus offen ist für spätere Erweiterungen.

Ausgangspunkt war dabei die Überlegung, die Vorteile des Methodenbankkonzeptes zu nutzen. Nachdem in den letzten 10 Jahren sehr leistungsfähige und preisgünstige Hardware und benutzerfreundliche Softwarestandards entwickelt wurden, rückte die DV immer näher an den Arbeitsplatz des Anwendungsfachmannes ohne spezielle DV-Ausbildung. Die Softwarekosten blieben verhältnismälig hoch, so daB der Aspekt der Nutzung vorhandener Software an Bedeutung gewann. Das Konzept der Methodenbank ermöglicht es, "vorgefertigte Bausteine dem Anwender als Programmonserven mit Datenbankschnittstelle so flexibel und benutzerfreundlich wie möglich anzubieten" (/PAGB4/). Ein Methodenbanksystem soll folgende Anforderungen erfüllen:

- Verwaltung auch großer Datenbestände, Datenselektion und Realisierung eines angemessenen Datenschutzes. Diese Aufgaben können von einer Datenbankkomponente übernommen werden.

- Definition, Verwaltung und beliebige Verknüpfung von "Methoden". Eine Methode ist hierbei die Realisierung eines Algorithmus in form eines abgeschlossenen programmes mit definierter Schnittstelle nach auBen ohne externe Referenzen (vgl. /PAGB4/).

- Integration der Datenbank- und Methodenbankkomponente in ein Rahmensystem, das dem Benutzer eine einheitiche, von den Subsystemen unabhängige oberfläche und Benutzerführung z.B. durch Menütechnik, Öberprüfung der Eingaben und Hilfstexte bietet.

In /TSC85/ wurde in Konzept oines interaktiven statistischen Auswertungs- und Modellierungssystoms, das sich am Methodenbankansatz oriontiert, vorgeschlagen. Dieses Konzept geht von einer Datenbank-, einer Methodenbank- und einer Modellbankkomponente aus, die in ein Rahmensystem integriert werden. Diese 
Arbeit untersucht, inwieweit sich dieses konzept zur Verarbeitung von Meßdaten, wie sie im Hamburger Luftmeßnetz anfallen, eignet. Auf den empfohlenen Einsatz von MBS als Modelibankkomponente wird verzichtet, weil MBS, das insbesondere sozio-ökonomische Modelibildung unterstützt, vom Benutzer umfangreiche Systemkenntnisse verlangt und sich bei unsachgemäBer Bedienung als sehr anfällig erweist (vgl. /HIL85/).

Ziel der Arbeit ist es nicht, ein system zu konzipieren, das den Anforderungen an eine Methodenbank in allen Punkten gerecht wird. Durch den Einsatz geeigneter Teilsysteme können jedoch wichtige Anforderungen erfüllt werden, ohne bej der Implementation den Rahmen zu sprengen. Eingesetzt werden:

\section{Ein Datenbanksystem (Scientific Information Retrieval system (SIR)). das insbesondere für die verwaltung statistischer 0aten im wissenschaftichen Bereich entwickelt wurde, das neben der langfristigen speicherung aller anfallenden Daten weitgehend beliebige Anfragen umsetzen kann und einen ausreichenden Datenschutz gewährleistet. \\ - Ein Statistikpaket (Statistical Analysis system (SAS)), das neben den Standardauswertungen auch Methoden für sehr komplexe Fragestellungen anbietet, über eine eigene Kommandosprache für selbst programmierte Auswertungen verfügt und die Entwicklung eigener Methoden mit einer schnittstelle zu höheren Programmiersprachen (PLI oder FORTRAN) unterstützt. \\ - Ein Menügenerator (Interactive Programming Facility (IPF)), der es ermöglicht, die teilweise komplexen systemaufrufe zu verdecken, Eingabefehler abzufangen, und dem Endbenutzer eine leicht verständiche oberiläche anbietet.}

Die Teilsysteme werden zu einem Gesamtsystem mit einheitlicher Benutzeroberfläche integriert. Das Gesamtsystem hat die folgende Struktur:

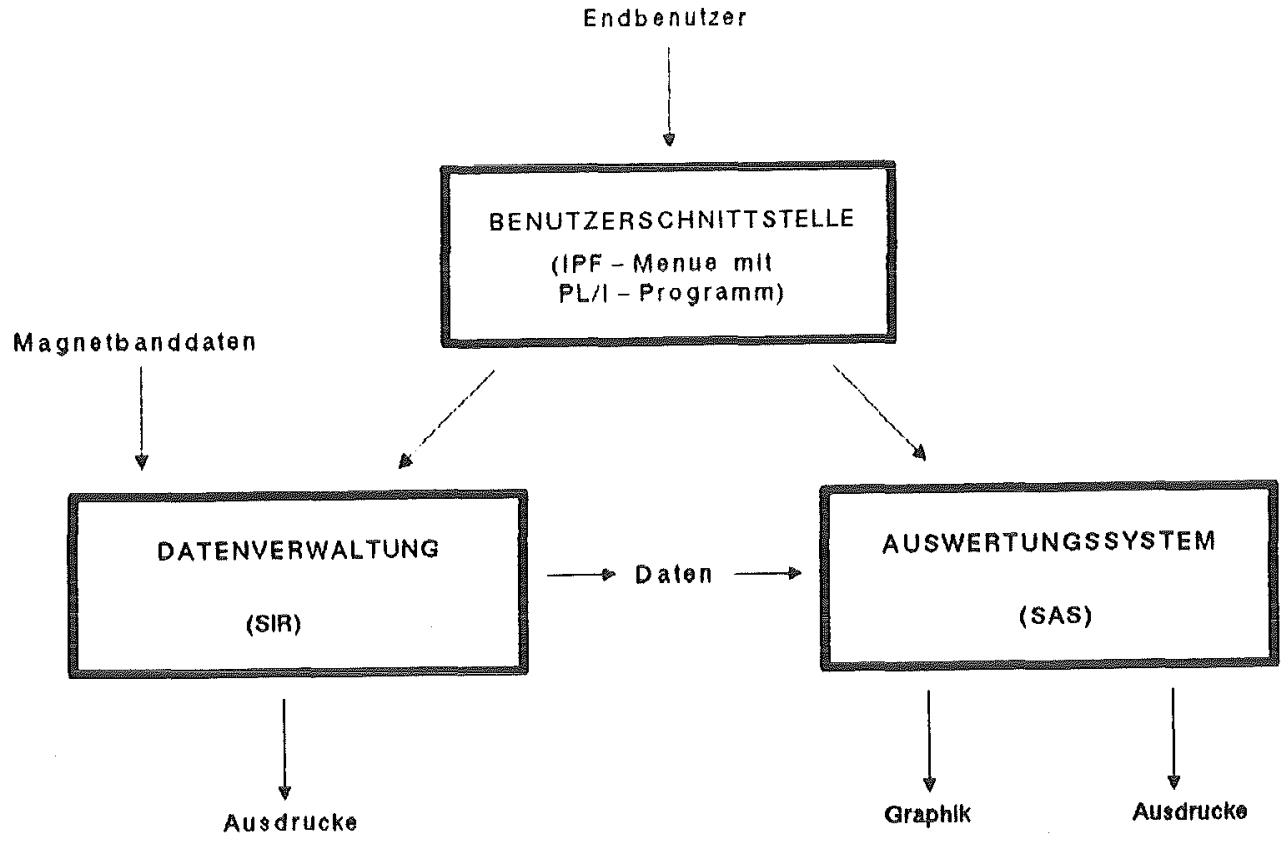

Abbildung 1: Struktur des Auswertungssystems 
In der Kombination der Systeme IPF, SIR und SAS liegen die folgenden besonderen Vorteile:

Die Standardschnittstelle von SIR zU SAS ermöglicht einen problemlosen Datentransfer. Da die Menüsteuerung durch IPF in eine universelle Programmiersprache eingebettet ist, können sowohl eine beliebig komfortable Benutzerführung als auch alle nötigen Konsistenzprüfungen realisiert werden. Mit Hilfe von IPF können Betriebssystemkommandos abgesetzt werden, das Menüsystem selbst ist vom verwendeten Betriebssystem unabhängig. Der Benutzer braucht so die Handhabung des Betriebssystems nicht zu erlernen.

Bei einer vollständigen Realisierung des konzipierten systems sollte es möglich sein, die Luftmeßaten nicht nur neu aufzubereiten und einen leichteren Zugriff $z u$ ermöglichen, sondern auch durch umfangreiche Auswertungen und übersichtliche graphische Darstellungen einen raschen überblick und tieferen Einblick in Zusammenhänge zu gewinnen. So ergeben sich bessere Möglichkeiten, die Ursachen der Luftverschmutzung zu analysieren und auf neue Entwicklungen schneller zu reagieren.

2.

Beschreibung des Auswertungssystems

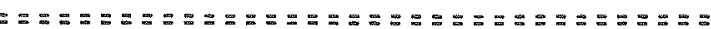

In diesem Kapitel wird das Auswertungssystem zunächst anhand eines Beispieldialoges aus der Sicht des Endbenutzers gezeigt. Nach einer Beschreibung der verwendeten Teilsysteme und des Rahmensystems wird genauer auf die Realisierung der Datenverwaltung und der statistischen Auswertungen eingegangen.

\subsection{Die Benutzersicht des systems}

\subsubsection{Die Bedienung des systems}

Der Endbenutzer steuert das system vollständig über Maskeneingaben im Dialog. Es ist lediglich die Eingabe der gewünschten Parameterwerte erforderlich, die sofort auf ihre Konsistenz hin geprüt werden. Bei falschen Eingaben erscheint eine Fehlermeldung, und der Cursor wird auf das zu korrigierende Feld positioniert. Da die Startmaske direkt nach dem Einloggen erscheint, benötigt der Endbenutzer keine Betriebssystemkenntnisse. Weitere Maskenseiten werden automatisch vom system angezeigt, sofern die Eingabe zusätzlicher Parameter erforderlich ist. Mit Hilfe einer Funktionstaste kann der Benutzer nach AbschluB einer Auswertung auf eine höhere Maskenhierarchieebene zurückkehren, um die Verarbeitung fortzusetzen.

Nach Systemstart kann der Benutzer auf einer Auswahlseite entscheiden, ob er eine neve 0atenbankanfrage wünscht, oder ob die zuletzt an das statistiksystem übergebenen Daten weiter ausgewertet werden sollen. Auf der Datenselektionsseite werden die Parameter für eine Datenbankanfrage eingegeben. Der Benutzer hat die Wahl, die selektierten Daten lediglich auszudrucken oder sie an das Statistiksystem zur weiteren verarbeitung zu übergeben. Falls letzteres gewünscht wird, erscheint die statistikauswahlseite mit einer übersicht der 
statistischen Auswertungen. Hier erhält der Benutzer auBerdem die Möglichkeit, für die jeweils gewünschte statistikfunktion nicht benötigte Komponenten von der Verarbeitung auszuschließen. Falls für die ausgewählte Auswertung weitere Angaben erforderlich sind, werden diese auf einer anschliebend angezeigten Unterseite eingegeben. Die Ausgabe erfolgt auf dem Drucker oder - bei Graphik auf dem Plotter.

Nach AbchluB einer Statistikauswertung kann der Benutzer eine weitere Auswertung für die selektierten Daten anstoßen und dafür gegebenenfalls andere Komponenten aktivieren, eine neue Datenbankanfrage anstoßen oder das system verlassen.

\subsubsection{Beispieldialog}

\section{1 .2 .1 Aufgabenstedlung}

Es soll der EinfluB des Autoverkehrs auf den Tagesgang der Luftbelastung untersucht werden. Hierzu wird der über einen Monat gemittelte Tagesverlauf der Belastung durch stickstoffmonoxid (Emission zu 54\% durch den Verkehr, nach IUBA84/) mit der Belastung durch Staub (Emission $249 \%$ durch den Verkehr) verglichen. Die Ausgabe erfolgt durch Plotten der durchschnittichen Tagesverlaufskurven.

\subsubsection{Selektion der benötigten Daten}

Nach dem Einloggen und dem Anwählen der entsprechenden Seite selektiert der Benutzer die gewünschten Daten (MeBstation: 14; Schadstoffe: No, N02 und Staub; Auswertungszeitraum: Juni 1985; Statistik: Ja) aus der SIR-Datenbank. Nach AbschluB aller Eingaben ist die 〈ENTER〉-Taste zu betatigen.

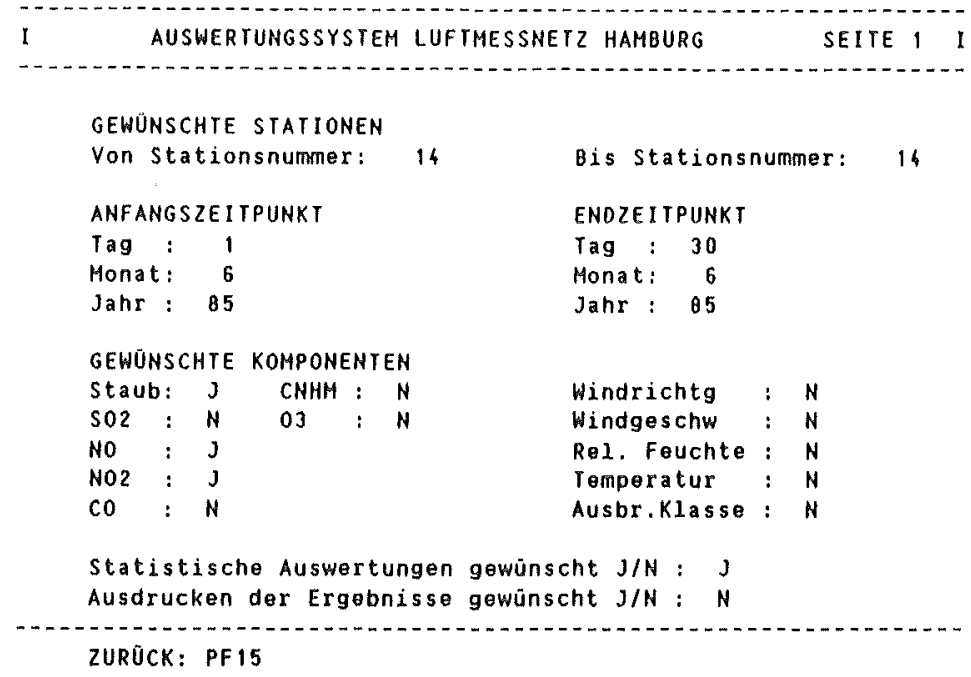

Abbildung 2: Datenselektionsmaske 


\subsubsection{Auswahl der statistikfunktion}

Da Statistikauswertungen gewünsht wurden, erscheint anschließend die
Statistikauswahlseite. Hier wäht der Benutzer die Funktion
Tagesverlaufskurven. aus, Das system zeigt nochmals die selektierten
Schadstoffkomponenten an. Der Benutzer wünscht zunächst keine Analyse der
No2-Werte und deaktiviert diese Komponente.

SELEKTIERTE KOMPUNENTEN
StaUb: J CNHA : N

\section{Abbildung 3: Statistikauswahlmaske}

\subsubsection{Eingabe zusätzlicher Parameter}

Auf der Seite 2.2 kann der Benutzer entschejden, ob einzelne oder gemittelte Tagesverlaufskurven ausgegeben werden sollen.

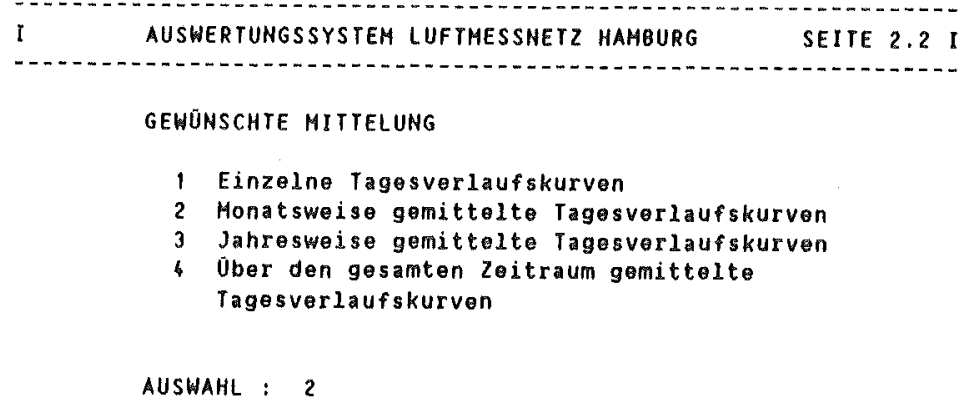

Übereinanderlegen der Kurven gewünscht $\mathrm{J} / \mathrm{N}: \mathrm{J}$

ZURÜCK: PF 15

Abbildung 4: Spezifikationsmaske für Tagesverlaufskurven 
2.1.2.5 Ergebnisausgabe

Die angestoßene Verarbeitung liefert folgende Ausgabe:

SCHADSTOFFBELASTUNG IM DURCHSCHNITTLICHEN TAGESVERLAUF ZEITRAUM 1. BIS 30 . JUNI 1986

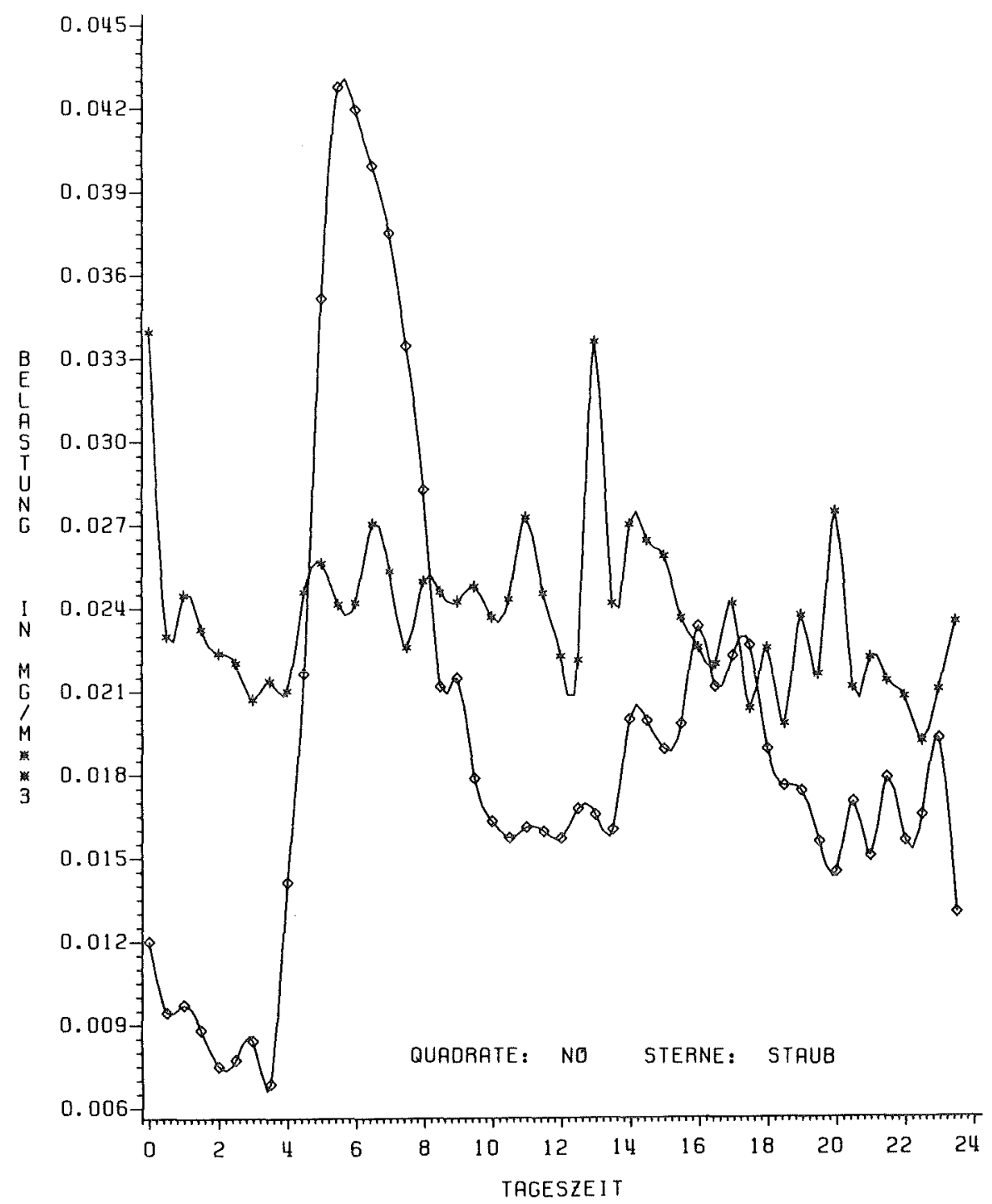

Abbildung 5: gemittelte Tagesverlaufskurven für staub und N02

Wie Abbildung 5 zeigt, ist insbesondere morgens ein kräftiger und nachmittags ein gemäBigter Anstieg der Schadstoffbelastung durch stickstoffmonoxid zu verzeichen, während die Tagesschwankungen der Staubkonzentration unregelmäßiger verlaufen und außerdem geringer sind. Es kann davon ausgegangen werden, daß die Stickstoffmonoxidbelastung durch das Verkehrsaufkommen (Berufsverkehr) wesentlich beeinflußt wird. 


\subsection{Die verwendeten systeme}

\subsubsection{Das Datenbanksystem SIR}

\subsubsection{Allgemeines}

Das Datenbanksystem SIR eignet sich besonders für die Speicherung analytisch zu bearbeitender 0 aten. Es besteht aus einem Grundsystem und den Erweiterungsmoduln SIR/FORMS (Maskengenerator). SIR/SQL+ (Datenbankanfragesprache SQL) Und SIR/HOST (Aufruf von $S I R$ aus einer höheren Programmiersprache). Im derzeitig eingesetzten SIR-Release 2.1.3. ist Mehrbenutzerbetrieb möglich.

\section{2 .1 .2 Das SIR-Grundsystem}

Das SIR-Grundsystem beinhaltet

- als Benutzerschnittstelle den 'SIR-Editor': hier kann der Benutzer direkt Kommandos an das $S I R-s y s t e m$ absetzen oder auch eine Kommandofolge (z.B. ein Datenretrieval) erstellen, die dann im 'Pseudo-Batch' (start eines asynchronen prozesses auf Betriebssystemebene) durchgeführt wird (das Grundsystem ermöglicht keine maskenunterstütze Suche und Ausgabe von Datenl;

- eine eigene, komfortable Retrievalsprache für die Datenselektion;

- begrenzte Statistik (Grundfunktionen, z. . deskriptive Statistik);

- einfache Graphik lDiagramme und Kurven mit Hilfe des Textzeichensatzesl;

- die Realisierung von Datenschutz mit verschiedenen Hierarchiestufen für Lese- und Schreibzugriffe bis hinunter zur Feldebene. SIR ermöglicht allerdings keine Definition von Anwendersichten;

- Integritätsprüfungen bei der Dateneingabe durch die Definition gültiger Werte und die Festlegung von Wertebereichen;

- Definition von bis zu drei sonderwerten, die als fehlende Werte interpretiert werden;

- Definition beschreibender Texte für Variablen und für Werte:

- eine Schnittstelle zu Standardstatistiksystemen wie SAS, BMDP und SPSS;

- Reporterstellung durch einen Reportgenerator.

\subsubsection{Datenorganisation und Datenselektion}

Das Datenbanksystem unterstützt insbesondere hierarchische strukturen. Als Datentypen stehen neben den standarddatentypen REAL, INTEGER Und STRING die Typen TIME, DATE und CATEGORICAL (Aufzählungstyp) zur verfügung.

oberster ordnungsbegriff ist der CASE. $Z u$ einem CASE können verschiedene Satztypen gehören. Ourch Definition der SORT IDS (Schlüssel) wird auch die hierarchische Einordnung der Sätze festgelegt. Durch geschickte Wahl der CASES kann die Effizienz von Eingabe- oder Retrievalläufen wesentlich gesteigert werden. Während eines Retrievallaufes werden spezifizierte Daten prozedural abgearbeitet. Gewünschte Variablenwerte werden dabei in einen 'Summary Record' übertragen, dessen Inhalt dann für die SIR-Standardprozeduren (z.B. Ausgabe, Graphik, Übergabe an SASI bereitgestellt werden kann. 


\subsubsection{Verwendung von SIR innerhalb des Auswertungssystems}

Das SIR-Grundsystem kann sehr effizient für batchorientierte Massendatenverarbeitung bei hierarchisch strukturierten Daten eingesetzt werden. Es erfült daher die ihm zugedachten Aufgaben innerhalb des Auswertungssystems. Schwächen, die sich bei einer dialogorientierten, kommerziellen Anwendung zeigen könten lbeispielsweise die geringe Flexibilität eines hierarchischen Datenmodelis), fallen in dieser Anwendung nicht ins Gewicht. Insbesondere entfallen sowohl komplexe Verknüpfungen der Daten untereinander als auch die Notwendigkeit der Definition vieler unterschiedlicher Satztypen. Es werden im wesentlichen nur MeBreihen fortgeschrieben und Anfragen an die Datenbank gestellt. Das Ändern oder Löschen von Datensätzen erfolgt nur in Ausnahmefällen.

\subsubsection{Das Statistikpaket SAS \\ 2.2 .2 .1 Allgemeines}

SAS bietet ein breites Angebot an Standardmethoden der Statistik, darunter auch komplexe Anwendungen wie multivariate Analyse und Regressionsanalyse. Eine sehr mächtige Erweiterung des SAS-Grundsystems ist das Modul SAS/GRAPH, mit dem anspruchsvolle, auch dreidimensionale Farbgraphik erzeugt werden kann.

\subsubsection{Das SAS-Basissystem}

SAS kann beliebige Eingabedateien (z.B. Lochkarten) verarbeiten, sofern deren Struktur dem system bekannt ist. Allerdings bietet das system keine Konsistenzprüfungen für die vorliegenden Daten. Die Kommandosprache ermöglicht Datenmodifikationen auf dem Niveau einer höheren Programmiersprache. Für sehr unterschiedliche Aufgabenbereiche existieren Statistik-Standardprozeduren. Die Ausgabe wird durch einen Reportgenerator unterstützt.

Der Anwender kann das SAS-system in unterschiedlicher weise nutzen:

- durch einfache Parametrisierung der SAS-Standardprozeduren,

- durch Programmierung in der SAS-Kommandosprache,

- durch Erstellen von selbst programmierten Methoden in einer höheren Programmiersprache (PL/I oder FORTRAN) und deren Einbindung in SAS.

\section{$2.2 .2,3$ Datenstruktur und Kommagdosprache}

Die in SAS verwendete Datenstruktur ist der SAS DATA SET, eine zweidimensionale Matrix mit bis zu 4000 Variablen und nur durch den vorhandenen speicherplatz begrenzten Beobachtungen. Die SAS-Kommandosprache besteht im wesentichen aus

- DATA STEPS: hier werden die Daten manipuliert, Berechnungen vorgenommen und neue Ausgabe-Datasets aus den Eingabe-Datasets erzeugt (z.B. Erzeugung eines verkleinerten Datasets durch Auswerten einer Bedingung oder Mischen zweier Eingabe-Datasets). 
- PROCEDURE STEPS: Anwendung von Methoden/Auswertungsfunktionen auf (vollständige) Datasets. Hier ist das Auswerten von Bedingungen vor Anwendung der Methode nicht mehr möglich.

\subsubsection{Verwendung von SAS innerhalb des Auswertungssystems}

Die von SIR erzeugte SAS-Ausgabedatei kann direkt als SAS-Dataset eingelesen werden. Für die meisten Verarbeitungsgänge genügen die SAS-Standardprozeduren. SAS bietet als offenes system die Möglichkeit, sehr spezielle, komplexe Auswertungen in einer höheren Programmiersprache selbst zu programmieren und sie dem System als neue Methode hinzuzufügen. Diese neuen Methoden werden dann vom system mit verwaltet und können vom Benutzer wie standardmethoden verwendet werden.

\subsubsection{Der Maskengenerator IPF}

\subsubsection{Al1gemeines}

IPF ermöglicht von einem Anwendungsprogramm aus den Aufruf von Betriebssystemkommandos und die Definition und Verarbeitung von Bildschirmmasken.

Das Programmpaket kann von FORTRAN, COBOL und PL/I aufgerufen werden. Durch Funktionen zum Teilen des Bildschirms oder zum Scrollen auf einzelnen Bereichen des Bildschirms ermöglicht IPF eine komfortable Bildschirmsteuerung. Auch kann ein Lichtgriffel für die systemsteuerung eingesetzt werden.

\subsubsection{Maskendefinition in IPF}

Die Implementation einer Bildschirmmaske mit Hilfe von IPF besteht aus einem Maskendefinitionsteil (eigene IPF-syntax) und einem Maskenverarbeitungsteil (Anwendungsprogramm).

Im Maskendefinitionsteil wird das ÄuBere der Bildschirmmaske (Ausgabetexte, verschiedene Helligkeitsstufenl festgelegt und die Maskenfelder mit Datentypen werden definiert. Im Verarbeitungsteil erfolgt durch den Einsatz verschiedener IPF-Prozeduren die Bildschirmsteuerung und der Aufruf verschiedener Masken. Der Einsatz einer höheren Programmierspache im Hintergrund eröffnet hier weitgehende Möglichkeiten, so können

- Benutzereingaben auf Konsistenz geprüft werden,

- Meldungen und Hilfstexte ausgegeben werden.

- Ausgabedateien für die steverung von im Hintergrund arbeitenden systemen erzeugt werden und

- Betriebssystemkommandos (zum Aufruf dieser systeme) abgesetzt werden.

Die IPF-Prozeduren liefern einen Return-Code zurück. Dieser dient nicht nur zum Erkennen und Behandeln von Fehlern, sondern gibt auch Aufschluß darüber, ob eine Funktionstaste gedrückt wurde. So können dem Benutzer bekannte Funktionstastenbelegungen (etwa Bildschirmsteuerung, Abbruch, Hilfstaste) im Anwendungsprogramm analog implementiert werden. 


\subsubsection{Verwendung von IPF innerhalb des Auswertungssystems}

Die von IPF zur Verfügung gestellte Maskentechnik erlaubt eine benutzerfreundliche Bedienung des Auswertungssystems. Besonders wichtig ist die Verdeckung von systemaufrufen, die dem Anwender eine aufwendige Einarbeitung in das jeweilige system erspart. Der Einsatz einer höheren Programmiersprache im Hintergrund ermöglicht eine komfortable Benutzerführung.

\subsection{Das Rahmensystem}

2.3.1. Randbedingungen des systemeinsatzes

Das Auswertungssystem ist auf dem Großrechner SIEMENS 7800 (IBM-kompatibe1) unter dem Betriebssystem BS3000 (MVS) implementiert. Voraussetzung für eine Portabilität des Auswertungssystems ist die Portabilitat der Teilssysteme. Der Einsatz der Teilsysteme kann auch unter anderen Betriebssystemen erfolgen, jedoch sind die mit Hilfe der Dialogkomponente abgesetzten Befehle spezifisch für dieses Betriebssystem.

\subsubsection{Die Aufgaben des Rahmensystems}

Das Rahmensystem soll die eingesetzten Teilsysteme steuern und den internen Datenverkehr zwischen ihnen kontrollieren. Der Endbenutzer benötigt keine Kenntnisse über die Einzelsysteme. Ihm wird eine einheitliche oberfläche angeboten, die von der oberfläche der Teilsysteme unabhängig ist. Der Aufruf der benötigten Teilsysteme ist verdeckt. Auch die Handhabung des Betriebssystems BS3000, die teilweise recht umständich ist und erhebliches spezialwissen erfordert, wird dem Endbenutzer abgenommen. Das Rahmensystem kontrolliert dabei auch den zeitlichen Ablauf der Aufrufe, so da z.B. keine Statistikauswertung angestoßen wird, bevor die entsprechende Datenbankanfrage abgeschlossen ist.

Eine besonders benutzerfreundliche Bedienung soll durch die einheitliche Maskengestaltung und die sofortige Konsistenzprüfung der Benutzereingaben ermöglicht werden.

Die Teilsysteme übernehmen innerhalb des Gesamtsystems streng abgegrenzte Funktionen. Ihre stärken sollen dadurch optimal ausgenutzt werden. Diese Funktionsaufteilung wird im folgenden Abschnitt beschrieben.

\subsubsection{Die Funktionsaufteilung innerhalb des Gesamtsystems}

Da der Benutzer die systeme SIR und SAS nicht direkt benutzt, sondern nur über das Programm mit ihnen kommuniziert, wurden die Funktionen Auskunftssystem und 'Benutzerführung/Konsistenzprüfungen' dem Programm übertragen. Hier muB auch der nötige Datenschutz realisiert werden, da das Programm die Zugriffsrechte auf die SIR-Datenbank hat.

Aus konzeptionellen Gründen (Trennung von Datenverwaltungs- und Auswertungsfunktionen) wurden Aufgaben aus den Gebieten Graphik und statistik dem sehr mächtigen system SAS übertragen, auch wenn Teilprobleme mit der SIR-Graphik und den SIR-Statistikfunktionen zu lösen gewesen wären. Für das Datenbanksystem bleibt nur die Datenverwaltung. 
Auskunftssystem

Datenschutz

Datenverwaltung

Statistik

benutzereigene

Hethoden

Graphik

Maskengenerator

Benutzerführung/

Konsistenzprüfungen $x$

$x$

$x$

Abbildung 6: Funktionsaufteilung innerhalb des Gesamtsystems

\subsubsection{Die Realisierung der Dialogkomponente}

\subsubsection{Die IPF-Menüdefinitionen}

Die Menüseiten werden in einzelnen Dateien definiert. Hier wird das äußere Erscheinungsbild der Maske sowie Lage und Datentyp der Eingabefelder festgelegt. Das Verarbeitungsprogramm liest dann bei Aufruf einer Menüseite die dazugehörige oatei ein. So können statische Informationen und Verarbeitung getrennt werden.

\subsubsection{Das PLI-Programm}

Das im Hintergrund eingesetzte PL/I-Programm übernimmt

- die Steuerung des Dialogablaufs und die Konsistenzprüfung der Benutzereingaben,

- die Festlegung der Parameter für Datenbankanfragen und Statistikauswertungen anhand der Benutzereingaben,

- das Erzeugen von Dateien, die die entsprechenden Datenbank- und Statistikaufrufe enthalten, und

- das Auswählen und Absetzen von Betriebssystemkommandos zum Aufruf der Teilsysteme. 
2.3.5. Der Datenfluß im Gesamtsystem

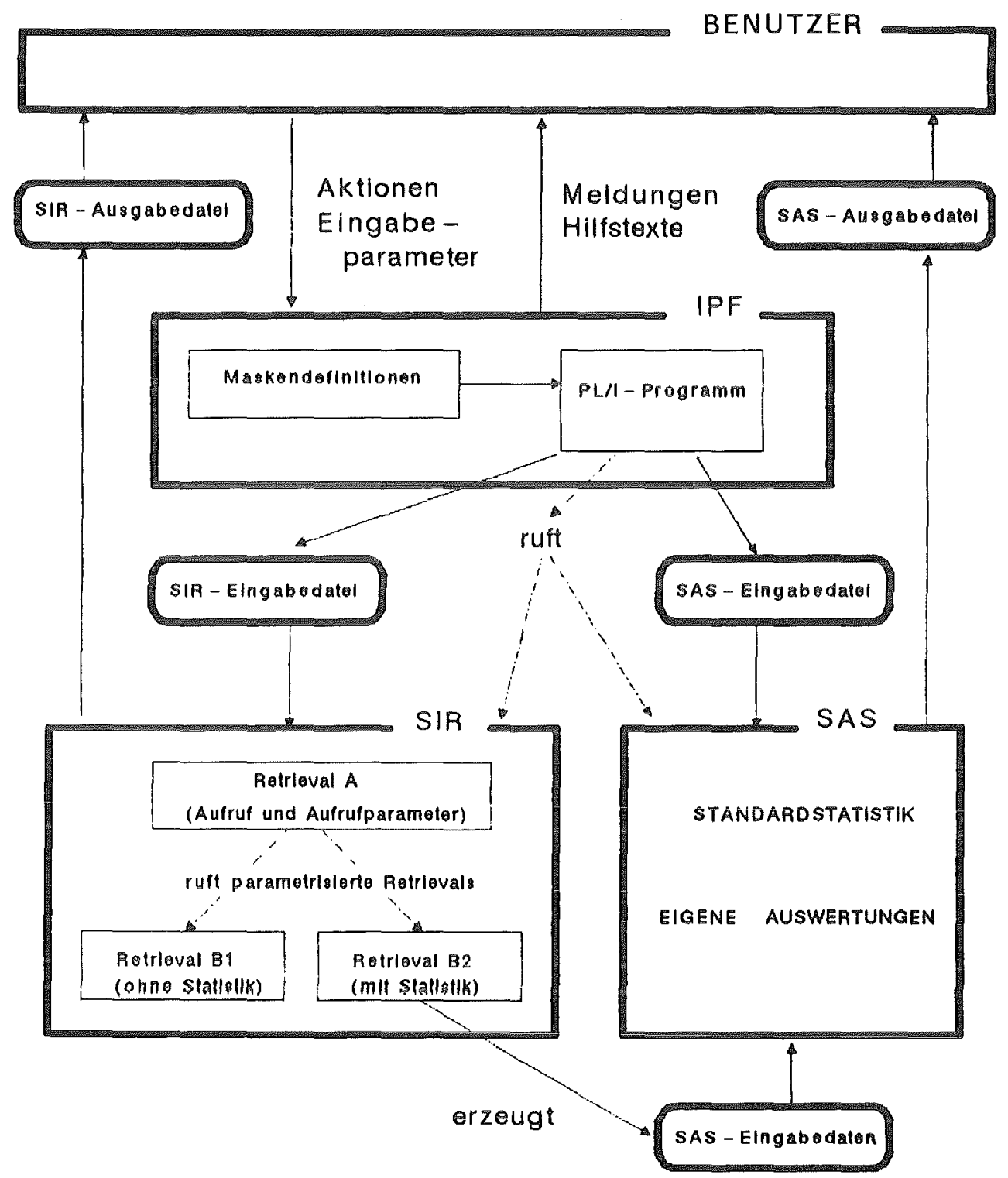

Abbildung 7: DatenfluB im Gesamtsystem

\subsection{Die Datenverwaltung}

2.4.1. Die Definition der Datenbank

\section{4 .1 .1 Vorliegende Daten}

Im Hamburger Meßnetz messen derzeit 23 Meßstationen täglich 48 Halbstundenwerte für bis zu sieben verschiedene Schadstoffkomponenten, je nach Gerätebestückung der Station. Darüber hinaus werden an zwei stationen ebenfalls halbstündich füf meteorologische Komponenten gemessen. 


\subsubsection{Die Schemadefinition in SIR}

Bei der Definition des Datenbankschemas ist dem bei Meßwertreihen typischerweise ungünstigen Verhältnis zwischen schlüsseln und Nutzdaten Rechnung zu tragen. Bei der Betrachtung der Daten drängt sich eine rein hierarchische organisation auf, da komplexe Verknüpfungen der Daten untereinander nicht erforderlich sind. Die vorliegenden Daten werden in folgende Hierarchiestufen eingeordnet:

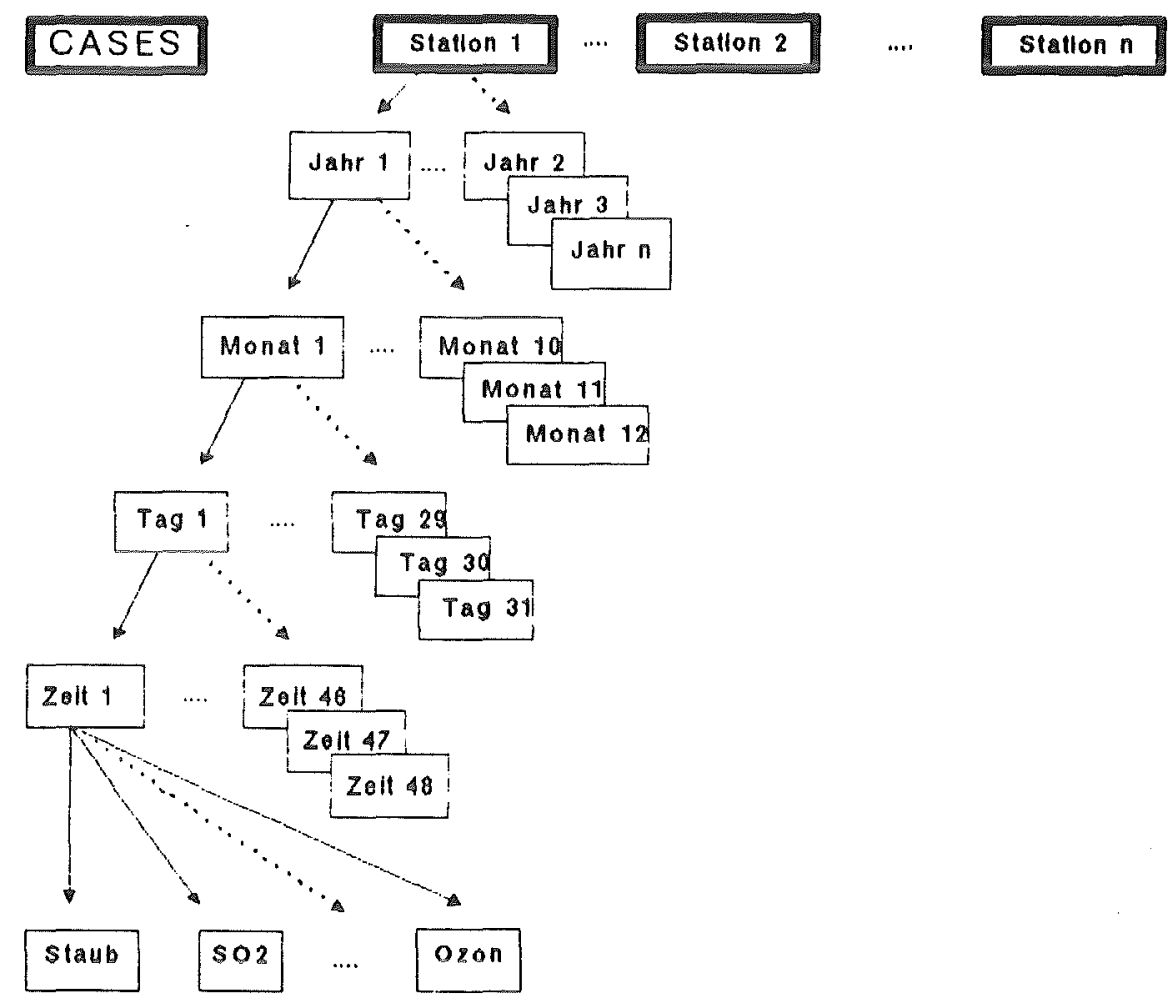

Abbildung 8: Datenhierarchie

Das hierarchisch orientierte Datenbanksystem SIR unterstützt derartige Strukturen. Es ist allerdings die vollständige Angabe der Schlüssel zu jedem Datensatz erforderlich, diese kann nicht durch die Definition von Hierarchiestufen ersetzt werden. Die physikalische Abspeicherung übernimmt SIR.

Da die Hauptaufgabe des Auswertungssystems darin besteht. Meßreihen zu einer oder mehreren Stationen auszuwerten, stehen diese als "CASES" in der obersten Ebene der Hierarchie. Es folgt die zeitliche Festlegung der Meßwerte durch Jahr. Monat, Tag und Zeit, wobei unter Zeit die 48 Halbstundenwerte zu verstehen sind.

Es werden zwei Satztypen definiert: Schadstoffdaten und meteorologische Daten. Die Schadstoffdaten haben als Schlüssel stationsnummer, Jahr, Monat, Tag und Zeit und sind als INTEGER definiert. Auf den ebenfalls zur Verfügung stehenden Datentyp DATE wurde verzichtet, da er eine Gruppierung nach Jahr, Monat oder Tag für bestimmte Auswertungen (z,B. Mittelwertbildung) nicht zuläBt. Für die Nutzdaten (Attributwerte) Staub, Schwefeldioxid, Stickstoffmonoxid, Stickstoffdioxid, Kohlenmonoxid, Kohlenwasserstoffe und ozon wurde aus Gründen der Platzersparnis der Datentyp SCALED INTEGER gewählt. Hier sind 5 Dezimalstellen ausreichend. 
Die meteorologischen Daten haben dieselben schlüssel. Nutzdaten sind hier Windrichtung, Windgeschwindigkeit, relative Luftfeuchtigkeit, Temperatur und Ausbreitungsklasse, die teils als INTEGER, teils als SCALED INTEGER definiert sind.

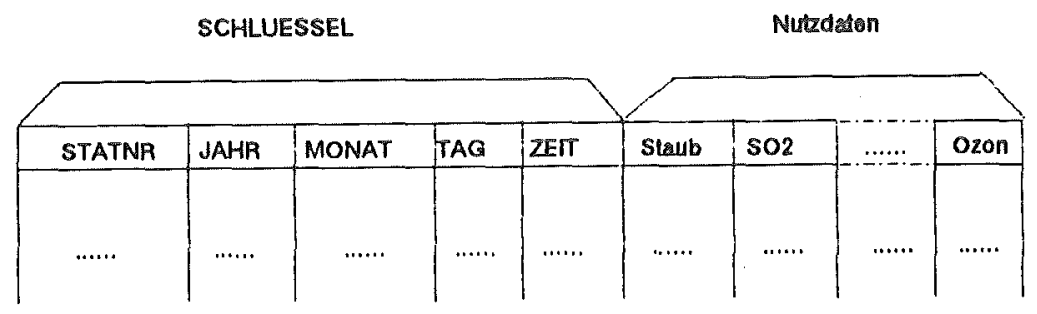

Abbildung 9: Darstellung des Satztyps "schadstoffdaten" in Matrixform

Die Hierarchiestufen werden durch die Reihenfolge der Schlüssel definiert. In diese Form müssen die Datensätze aufbereitet werden, bevor sie von dem Datenbanksystem eingelesen werden können (siehe Kap. 2.4.2.). Auf eine Verschmelzung der Schadstoff- und Meteorologiedaten zu einem Satztyp, die aufgrund der gleichen Schlüssel möglich wäre, wird verzichtet, da in der Datenmatrix die Felder für Meteorologiedaten bei fast allen Stationen leer bleiben müßten und so der Platzbedarf um ca. 50\% höher läge. Um andererseits ein akzeptables Verhältnis von schlüsseln zu Nutzdaten zu erhalten, werden die Schadstoff- und die Meteorologieparameter in joweils einem satztyp zusammenge faßt.

\subsubsection{Diskussion alternativer Schemadefinitionen}

Ein optimaler Zugriff auf den einzelnen Meßwert kann erreicht werden, wenn die betreffende Komponente ebenfalls als Schlüssel definiert wird. Damit werden Stationsnummer, Jahr, Monat, Tag, Zeit und Komponentennummer zu Schlússeln, Nutzdatum ist nur noch der MeBwert. Wegen des ungünstigen Verhältnisses von Schlüsseln zu Nutzdaten ist der Platzbedar bei dieser Lösung mehr als doppelt so hoch.

Die Abspeicherung aller Meßwerte eines Tages in einem Satztyp mit den Schlüsseln Stationsnummer, Jahr, Monat, Tag, Komponentennummer und den 48 Meßwerten als Nutzdaten bringt zwar Platzersparnisse, lä t aber eine Auswertung nach Zeitpunkten (z.B. den Vergleich der Schadstoffbelastung zu Zeiten des Berufsverkehrs mit zeiten geringerer Belastungl nicht zu. Außerdem legt sie sich starr auf 48 Halbstundenwerte fest. Da eine Parametrisierung innerhalb der Felder eines satztyps nicht möglich ist, müßten die $4 \theta$ Werte jeweils einzeln behandelt werden.

\subsubsection{Platzbedarf der Daten}

Die auf dem Magnetband des Hamburger LuftmeBnetzes erfaßten Rohdaten nehmen pro Monat $1.25 \mathrm{MB}$ ein. Bei einem Füllungsgrad von $100 \%$ belegen diese Daten in der Datenbank $1.65 \mathrm{MB}$, davon $0.84 \mathrm{MB}$ für die Abspeicherung, den Rest für die Datenverwaltung. Für statische verwaltungsinformation werden weitere $0.2 \mathrm{MB}$ benötigt, die nicht von der Menge der gespeicherten Daten abhängig sind. Ein 
voll ausgebautes system benötigt damit ca. 20 MB pro Jahr, was nicht wesentlich über dem Platzbedarf der Rohdaten liegt. Diese sehr effiziente Platznutzung erklärt sich hauptsächlich durch die verwendung des Datentyps SCALED INTEGER (statt REAL) und die implizite Abspeicherung der Markierungen als MISSING VALUES. Es kann hier mit vollem füllungsgrad gearbeitet werden, da keine Änderungen an den gespeicherten Daten anfallen und die Menge neu hinzukommender Daten bekannt ist, so daß die GröBe der Datenbank darauf abgestimmt werden kann.

Bei begrenztem Plattenspeicherplatz bietet SIR die Möglichkeit, die Datenbank auf Magnetband auszulagern und für Auswertungen im Batch zu verwenden, was insbesondere für die seltene Verarbeitung sehr großer Datenmengen vorteilhaft ist.

\subsubsection{Flexibilität der Datenbankdefinition gegenüber Erweiterungen}

Das Hinzufügen never Stationen und das Abspeichern von Werten zu anderen Meßzeiträumen ist problemlos möglich. Die Hinzunahme von Komponenten an einzelnen stationen bereitet auch keine Schwierigkeiten, sofern es sich um eine der vordefinierten Komponenten handelt. Die Erweiterung des Meßspektrums erfordert allerdings eine Modifikation des Datenbankschemas.

\subsubsection{Die Datenkonvertierung}

Die auf den Magnetbändern des Luftmeßnetzes gehaltenen Daten liegen in folgender Form vor:
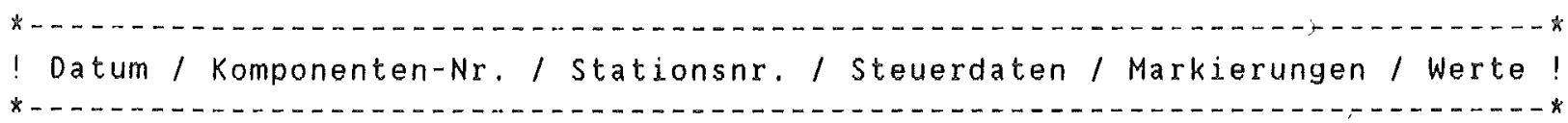

Mit Hilfe eines FORTRAN-Programmes werden diese Daten aufbereitet und in eine Eingabedatei für SIR geschrieben. Das Programm

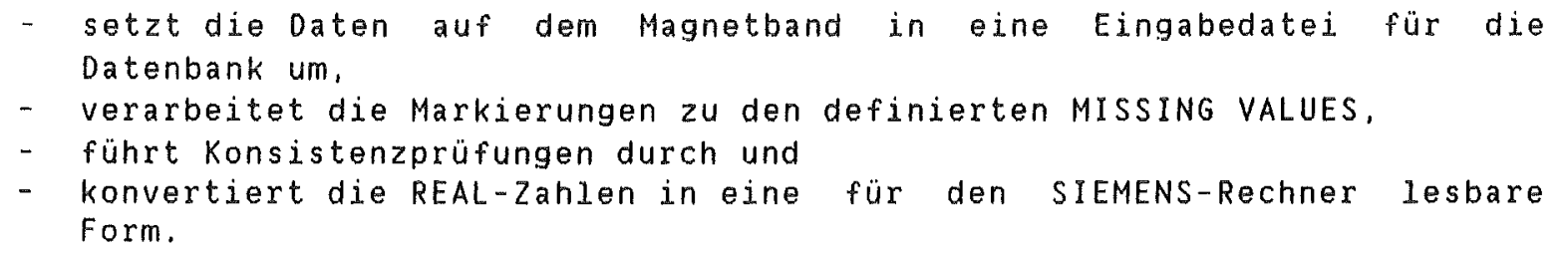

Dafür werden ca. $30 \mathrm{CPU}-$ Sekunden pro verarbeitetem Monat benötigt.

\subsubsection{Die Dateneingabe in SIR}

Nach der Vorverarbeitung der Eingabedaten durch das FORTRAN-Programm werden die Datensätze in die Datenbank übernommen. Das Einlesen der Eingabesätze für einen Monat erfordert ca. 180 CPU-Sekunden. Besonders zeitintensiv ist dabei die interne Umwandlung der reellen Zahlen in die entsprechenden SCALEO INTEGER Zahlen. Aus sicherheitsgründen empfiehlt es sich, die Batch-Eingabe großer Datenmengen in mehreren Teilen durchzuführen, da eine Unterbrechung des Eingabelaufes zu Schäden an der Datenbank führen kann. 


\subsubsection{SIR-Retrievals}

Um eine möglichst hohe flexibilität zu erreichen, werden für standardanfragen zwei allgemeine, parametrisierte Retrievals, die im SIR-System gespeichert sind, eingesetzt. Diese Retrievals werden mit folgenden Parametern aufgerufen:

- Stationsnummer-von, Stationsnummer-bis (bei Gleichheit der beiden Werte wird das Retrieval nur für die eine stationsnummer ausgeführt),

- Anfangszeitpunkt (Tag, Monat, Jahr),

- Endzeitpunkt (Tag, Monat, Jahr),

- gewünschte Komponenten (Auswahl aus Staub, SO2, No, NO2, CO, CNHM, 03, Windrichtung. Windgeschwindigkeit, Relative Luftfeuchtigkeit, Temperatur und Ausbreitungsklassel

- Statistische Auswertungen gewünscht Ja/Nein.

Eine Datenbankanfrage liefert für das Intervall von stationsnummern und den festgelegten Zeitraum die Schlüsselinformation und die Werte der ausgewählten Komponenten. Wünscht der Benutzer keine statistischen Auswertungen, werden die selektierten Werte in eine SIR-Ausgabedatei geschrieben. Andernfalls werden für die Stationen zusätzlich die Meteorologiewerte der jeweils zugeordneten Meteorologiestation bereitgestellt. Die ausgewählten Daten werden dann auf eine 0 atei geschrieben, die vom SAS-System weiterverarbeitet werden kann.

\subsection{Statistische Auswertungen}

\subsubsection{Datenübergabe von SIR an SAS}

Mit Hilfe einer SAS-Standardprozedur erfolgt die Datenübergabe von SIR an SAS. Es werden alle Variablenausprägungen, die während des SIR-Retrievallaufes spezifiziert wurden, in eine für SAS lesbare Datei geschrieben. Diese 0atei kann dann in SAS direkt als SAS DATA SET angesprochen werden.

\subsubsection{Auswertungen mit Hilfe der SAS-Statistikprozeduren}

Die auf der Statistik-Maskenseite aufgeführten Auswertungen werden ausnahmslos mit den SAS-Standardprozeduren und der SAS-Kommandosprache realisiert, Der Benutzer kann unter folgenden Auswertungen auswählen:

- Standardauswertungen,

- Tagesverlaufskurven,

- Schwellwertüberschreitungen.

- Perzentile.

- Korrelationen,

- Regressionen und

- Meteorologiebezogenen Auswertungen.

Außerdem hat der Benutzer die Möglichkeit, die Verarbeitung auf eine Untermenge der selektierten Komponenten zu beschränken. Im folgenden werden wir einige Auswertungen exemplaxisch beschreiben. 


\subsubsection{Standardauswertungen}

Der Benutzer kann unter folgenden Standardauswertungen auswählen:

- Zahl der zugrundeliegenden Meßwerte.

- Zahl der fehlenden Meßwerte.

- Mittelwert,

- Standardabweichung,

- Minimalwert und

- Maximalwert.

Er kann auBerdem angeben, ob und wie der Auswertungszeitraum zu gruppieren ist (tages-, monats- oder jahresweise) und ob die Ausgabe auf den Drucker geleitet oder am Bildschirm angezeigt werden soll. Die angeforderten Statistiken werden in tabellarischer Form ausgegeben. Eine graphische Ausgabe in Form von Säulendiagrammen ist hier ebenfalls denkbar.

\section{5 .2 .2 Korrelationen}

Auf der entsprechenden Masken-Unterseite dieser Auswertung gibt der Benutzer an, welche Parameter miteinander korreliert werden solien. Es stehen mehrere Korrelationsverfahren zur Auswahl. Eine Gruppierung der MeBdaten ist möglich. Die Ausgabe kann in Reihenfolge des betragsmäBig jeweils nächstgrößten Korrelationskoeffizienten erfolgen. Weiterhin kann eine graphische Darstellung der Ergebnisse angefordert werden.

Bei graphischer Ausgabe werden die gebildeten Wertepare mit Hilfe einer SAS/GRAPH-Standardprozedur auf einem Plotter ausgegeben. Ansonsten erfolgt eine tabellarische Ausgabe der berechneten Statistiken.

\subsubsection{Meteorologiebezogene Auswertungen}

Bei dieser Auswertung kann der Benutzer für die gewünschte Komponente Meß̧werte herausfiltern, die bei ganz bestimmten meteorologischen Verhältnisse auftreten. Hierbei können statistiken für ein Windrichtungsintervall, ein Windgeschwindigkeitsintervall, einen bereich relativer Luftfeuchtigkeit, oinen Temperaturbereich oder eine bestimmte Ausbreitungsklasse erstellt werden.

oiese Auswertung kann nur für eine Komponente zur zeit durchgeführt werden. Werden auf der Statistikauswahlseite mehrere Komponenten ausgewählt, erscheint eine Fehlermeldung, die den Benutzer auffordert, die Auswahl auf eine Komponente zu beschränken. Die erzeugten Statistiken werden in tabellarischer Form ausgegeben. Auf Wunsch des Benutzers können die Ergebnisse gedruckt werden.

\subsubsection{Einbettung benutzereigener Methoden in SAS}

Wie bereits in Kapitel 2.1. beschrieben, können auch benutzereigene Methoden in SAS verfügbar gemacht werden. Das Verfahren kann in diesem Rahmen allerdings nicht dargestellt werden. Diese Methoden können dann auf der Statistikauswahlseite mit zur Auswahl gestellt und vom Benutzer in gleicher Weise wie die anderen Auswertungen verwendet werden. 
Wie die Smogkatastrophe in London im Jahr 1952 zeigte, kann eine starke Belastung der Luft mit schadstoffen nicht nur zu gesundheitlichen schäden, sondern sogar zum Tod führen. Bei zahlreichen Krankheiten werden epidemiologische Kausalzusammenhänge mit erhohten Schadstoffkonzentrationen in der Atemluft vermutet, so zum Beispiel bei Lungen- und Bronchialkrebs und bei Pseudokrupp. Es erscheint uns deshalb wichtig, dieses Auswertungssystem auch zur Untersuchung von Korrelationen zwischen Daten über die Luftbelastung und epidemiologischen Daten einzusetzen.

Bei der Untersuchung derartiger Korrelationen ist $z u$ beachten, dab das vermehrte Auftreten einiger Krankheiten (z.B. Pseudokrupp. Herzanfälle) vermutich mit extremen Belastungen der Luft (Inversionswetterlagen. Smog) zusammenhängen, andere dagegen durch langfristig erhöhte Belastungen begünstigt werden (Lungen- und Bronchialkrebs).

\subsection{Datenmaterial}

Von einigen Krankheiten (z,B. Pseudokrupp) sind insbesondere Kinder betroffen, bei anderen Krankheiten (z.B. Herzanfälle) hauptsächlich ältere Menschen. Deshalb ist bei den $z u$ verwendenden Daten die bevölkerungsstruktur des Gebietes, in dem die Daten erhoben werden, relevant.

Da außerdem vermutlich insbesondere Belastungsspitzen das akute Auftreten einiger Krankheiten (2.B. Pseudokrupp) zur Folge haben, ist deshalb die prozentuelle Zu- oder Abnahme der Zahl der Krankheitsfälle pro 100000 Einwohner (bezogen auf langfristige Mittelwerte) mit Tages-spitzenwerten (95- oder 98-Perzentile) der Belastung durch bestimmte Luftschadstoffe zu korrelieren.

Besondere Aufmerksamkeit ist dabei der Qualität der bisher nicht standardmäBig erfabten epidemiologischen Daten zu schenken. Bei akut auftretenden Krankheiten werden schon von interessierten Ärten Statistiken (insbesondere für Pseudo-Krupp) geführt.

Eine Erweiterung des Auswertungssystems zur Korrelation dieser Daten mit den im Hamburger Luftmeßnetz seit 1965 regelmäßig anfallenden Daten zur Luftbelastung erscheint uns besonders interessant. Eine Einbeziehung langfristig entstehender Krankheiten dagegen halten wir aufgrund des fehlenden Datenmaterials derzeit nicht für sinnvoll.

oie Konzeption einer entsprechenden Erweiterung des Auswertungssystems wird im folgenden skizziert.

\subsection{Speicherung der epidemiologischen Daten in einer SIR-Datenbank}

In der obersten Hierarchieebene der SIR-Schemadefinition stehen als "CASES" die Regionen (entweder Meßquadrate oder grüßere Flächen, z.B. jeweils 4 Quadrate zusammengenommen). Analog zu den Luftdaten folgt die zeitliche Einordnung der Meßdaten nach Jahr, Monat und Tag. Ein zu definierender Satztyp ist z.B. der Satztyp "akut auftretende Krankheiten". Dieser enthält als Schlüssel die Regionsnummer (bzw. Stationsnummer), Jahr, Monat und Tag und als Nutzdaten die prozentuelle Zu- oder Abnahme der Zahl der Krankheitsfälle pro 100000 Einwohner für verschiedene Krankheiten an dem jeweiligen Tag. 


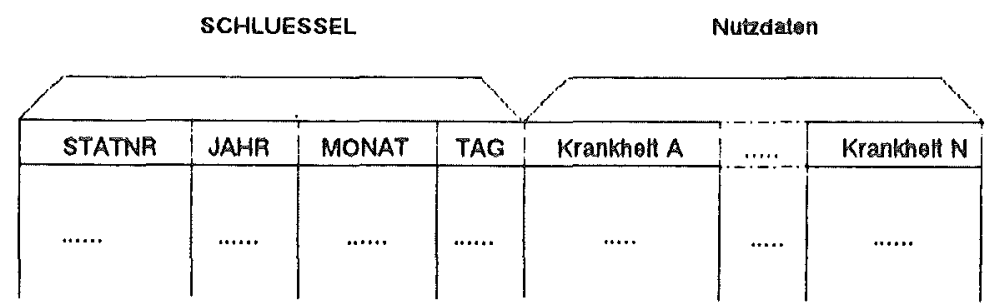

Abbildung 10: Satztyp "akut auftretende Krankheiten" in Matrixform

Eine horizontale speicherung der einzelnen Krankheiten bewirkt ein günstiges Verhälthis von schlüsseln zu Nutzdaten und vereinfacht die Korrelation mit den Schadstoffaten.

\subsection{Verarbeitung bei akut auftretenden Krankheiten}

Zunächst sind mit Hilfe bestimmter SAS-Standardprozeduren perzentile der Belastung mit einem bestimmten Schadstoff (90-, 95- oder $98-$ Perzentile) in einer bestimmten Beurteilungsfläche an dem zu untersuchenden Tag zu ermitteln. Das Ergebnis der angestobenen Prozedur wird in einem SAS DATA SET gespeichert.

Zur Vorbereitung der Korrelation wird dieser DATA SET mit einem DATA SET, der die epidemiologischen oaten enthält, anhand der gemeinsamen Schlüssel (Stationsnummer(Regionsnummer), Jahr, Monat, Tag) zusammengesetzt. Bei der Korrelation ist $z u$ beachten, daß fehlende werte nicht in die verarbeitung einbezogen werden dürfen.

Das Ergebnis der Korrelation, das wiederum in einem SAS DATA SET abgespeichert wird, dient als Eingabe für eine SAS/GRAPH-Prozedur, mit deren Hilfe die Korrelationskoffizienten und die Konfidenzbereiche anschaulich dargestellt werden können.

\subsection{Erweiterungsmöglichkeiten}

Bei sehr langfristig und multikausal entstehenden Krankheiten wie Lungen- oder Bronchialkrebs ist die Untersuchung des Einflusses der Luftbelastung wesentlich schwieriger. Hierbei ist die prozentuelle Zu- oder Abnahme von Krankheitsfällen pro 100000 Einwohner (bezogen auf einen mehrjährigen Durchschnitt) mit langfristigen Mittelwerten der Belastung durch bestimmte Luftschadstoffe aus einem weit zurückliegenden zeitraum zu korrelieren.

Da die systematische Messung von Luftbelastungsdaten erst seit einigen Jahren betrieben wird, steht heute auf diesem Gebiet kein entsprechendes Datenmaterial zur Verfügung. Die heute gemessenen Daten können in 10 bis 20 Jahren für derartige Untersuchungen herangezogen werden. Deshalb ist es wichtig, die jetzt anfallenden Daten für entsprechende Verarbeitungen verfügbar zu halten.

Die benötigten epidemiologischen Daten sind auch nicht in ausreichendem Maß vorhanden. Der Aufbau eines bundesweiten Krebsregisters wird dazu beitragen, hier Abhilfe zu schaffen. 
4.

Zusammenfassung und Ausblick

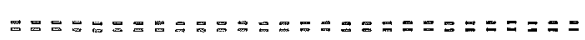

Der Einsatz der EDV auf dem Umweltsektor wird zunehmend wichtiger. Sie wird zu einem unentbehrlichen Hilfsmittel bei der statistischen Auswertung von Massendaten und bei der Verknüpfung von Daten aus Umweltbereichen mit Daten aus angrenzenden Gebieten.

Nur ein möglichst flexibles, offenes system ermöglicht es, auf neue Auswertungsanforderungen rasch zu reagieren. Es hat sich gezeigt, daß die Methodenbankarchitektur eine wichtige Grundlage für die konzeption eines Auswertungssystems darstellt. Die Verwendung leistungsfähiger subkomponenten erlaubt es dabei, den Implementationsaufwand auf ein vertretbares Mab zu reduzieren.

Im Rahmen dieses Projektes ist geplant, auch andere Datenbanksysteme, die keine Standardschnittstelle zu SAS haben, auf ihre Eignung als Datenbankkomponente innerhalb des Auswertungssystems $z u$ untersuchen. Hierbei wird einerseits auf ORACLE eingegangen, ein modernes, dialogorientiertes relationales system, das auch auf $\mathrm{CC}^{\prime} s$ einsetzbar ist, und andererseits ADABAS betrachtet, das derzeit vor allem im Behördenbereich eine breite Anwendung findet.

Ein besonders interessanter Gesichtspunkt ist der Einsatz des hier konzipierten Auswertungssystems auf $P C^{\prime} s$. Sowohl für die Datenbankkomponente SIR als auch für die Methodenbankkomponente SAS sind PC-Versionen 1 $Z$. B. für IBM-PC $X T, A T$ und kompatible) verfügbar. Die Dialogkomponente wäre systemspezifisch auszuwählen. Zwar kañn der Einsatz eines Grobrechners so nicht ersetzt werden, aber es könnten Teildatenbestände (z.B. Oaten für ein halbes Jahr) dezentral statistisch ausgewertet werden. Über ein Rechnernetz könnten die $P C^{\prime} s$ dann an eine zentrale Datenbank angeschlossen werden.

\section{Literaturverzeichnis}

IDIT79/ Dittrich, K. R., Hüber, R., Lockemann, P. C., Methodenbanksysteme: Ein Werkzeug zum Maßschneidern von Anwendersoftware, in: Informatik-Spektrum 2, 1979, S. 194-203 (Springer Verlag, 1979)

/HAN85/ Hansen, A., Zacharias, U., Automatische Luftmeßnetze (Studienarbeit am Fachbereich Informatik, Universität Hamburg, 1985)

/HAN86/ Hansen, A., Zacharias, U., Automatische LuftmeBnetze, in: Informatik im Umweltschutz - Anwendungen und Perspektiven, Hrsg. Prof. Dr.-Ing. Bernd Page unter Mitarbeit von Dipl.-Inform. Lorenz M. Hilty, S. 65-100 (München, Wien: R, Oldenbourg Verlag, 1986)

/HILB5/ Hilty, L., Benutzergerechte Modellierungssysteme - Kriterien für eine benutzergerechte Gestaltung von systemen zur Unterstützung der Modellbildung und Simulation (Diplomarbeit am Fachbereich Informatik. Universität Hamburg, 1985l 
/IPFB4/ Siemens system 7.800

operating system BS3000 MSP

IPF Interactive Programming Facilities

User's Guide $(1984)$

/PAG84/ Vorlesungsskript der Veranstaltung Anwendungen der Informatik: Modellbildung und Simulation (Universität Hamburg, SS 1984)

/SAS81/ SAS Institute Inc., SAS/GRAPH User's Guide

(Cary, North Carolina 27511, 1981)

/SAS82a/ SAS Institute Inc., SAS User's Guide: Basics

(Cary, North Carolina 27511, 1982)

/SAS82b/ SAS Institute Inc., SAS User's Guide: Statistics

(Cary. North Carolina 27511,1982 )

/SIR80/ Scientific Information Retrieval Inc.,

SIR User's Manual Version 2 (Evanston, IL 60204, 1980)

/SIR82/ Scientific Information Retrieval Inc.

SIR/DBMS 2.1.1

Data File size Estimates

EXPORT/IMPORT Utilities

Stored Executable Retrievals

SIR/DBMS Sequential Databases Technical Report

(Evanston, IL 60204, 1982)

/SIR83/ Scientific Information Retrieval Inc., SIR/DBMS 2.1 .2

IBM/OS Machine Dependencies (Evanston, IL 60204, 1983)

/SIR84/ Scientific Information Retrieval Inc., SIR/DBMS Version 2.1.3

Manual Supplement (Evanston, IL 60204, 1984)

/TSC85/ Tschichholz, G., Ein softwarekomzept für ein interaktives statistisches Auswertungssystem bestehend aus einer Datenbank-, Methodenbank - und Modellbankkomponente lDiplomarbeit am Fachbereich Informatik, Technische Universität Berlin, Institut für Quantitative Methoden, 1985)

/UBA84/ Umweltbundesamt (Hrsg.), Daten zur Umwelt 1984 (Berlin, 1984) 
Ansätze für Expertensysteme in der Unweltverträalichkeitsarüfung

\author{
Angela Schwabl \\ Universitat Hamburg \\ Fachbereich Informatik
}

\begin{abstract}
Zusammenfassung:
Planung, Verwaltung und Prüfung von Eingriffen in die Umwelt sind nur schwer vergleichbar und damit auch schuer durchschaubar. Je nach Verfügbarkeit und Uorliebe werden unterschiedliche Methoden und Instrumente eingesetzt.
\end{abstract}

Die Einführung einer ginheitlichen Umweltverträglichkeitsprüfung soll hier Abhilfe schaffen. Uber die Ausgestaltung des Uerfahrens Umweltverträglichkeitsprüfung herrscht jedoch keine Klarheit.

Die Informatik als Methodenwissenschaft kann hier Hilfestellung geben. Es wird aufgezeigt, wie Expertensysteme bei der Festlegung des Anwendungsbereiches einer UUP eingesetzt werden können und es werden Uberlegungen $z u$ ihrem Einsatz bei der Durcheührung der UuP angestellt.

\title{
GIiederung
}

1. Einleitung

2. Die Umweltverträglichkeitsprüfung

3. Uberlegungen zum Einsatz von Expertensystemelementen in

einem entscheidungsunterstützenden System UUP

3.1. Die Wissensbasis

3.1.1 Wissensdarstellung

3.1.2 Die Wissensbasis in einem EUS UUP

3.2. Die Problembehandlungskomponente

3.2.1 Inferenzkomponente und Kantrallstrategie

3.2.2 Eine Problembehandlungs-Komponente Für ein EUS UUP

3.3. Die Erklärungskomponente

3.3.1 Technik und Methoden Eür Erklärungskamponenten

3.3.2. Die Erklärungskomponente Eür ein EUS UUP

4. Konzept für ein regelarientiertes Expertensystem zur Unterstützung der Eingrenzung des Anwendungsbereichs

5. Literatur 


\section{Einleitung}

Die DECD hat festgestellt, daß die dffentliche Hand in den Mitgliedsländern etwa 1 - $2 \%$ des Bruttosozialprodukts Eür Umweltschutzaufgaben ausgibt, es jedoch $3-5 \%$ des

Bruttosozialprodukts kostet, bereits eingetretene Schädigungen der Umwelt wieder zu beseitigen.

Beispiel: Die Sanierung der in den 60er und 70er Jahren entstandenen Müllkippen und ungeordnetan Deponien erfordert Milliardenbeträge. Sie ist notwendig, da von solchen Deponien langfristig unerträgliche Gefahren ausgehen.

Es ist unstrittig, daß dieses Mißverhältnis überwunden werden muß.

Ein Werfahren zur Unterstützung einer vorsorgenden Umweltpolitik ist die Umweltverträglichkeitsprüfung (UUP), der Eingriffe in die Umwelt schon in der Planungsphase unterwarfen werden sallten. Notwendig ist die konsequente Durchsetzung einer UUP, die immer auch eine Ablehnung des Eingriffs als Ergebnis haben kann, wenn das geprüfte Uorhaben umueltzerstb̈rend wirkt. Nach Lage der Dinge ist diese Funktion einer UUP jedoch unrealistisch. Realistisch ist die Annahme, die UUP eigne sich als Verfahren, den Bereich Umwelt in einer durchschaubaren, systematisch aufgearbeiten Weise in den Entscheidungsprozeß über ein relevantes Varhaben einzuführen und der akologie damit eine (gleichberechtigte) Ralle neben der bkonomie und der Ordnungspolitik einzuräumen. Eine UUP als etabliertes VerEahren wird Maßstabe und Leitlinien für den Umgang mit der Umwelt prägen.

Es gibt sehr unterschiedliche Anwendungsbereiche der UUP. Nicht nur konkrete Bauvorhaben (häufigster Anwendungsfall in der Vergangenheit: Straßenbaul sind zu überprüfen, ebenso kann eine UUP $z . B$. auf den verfolgten oder alternative "Energiepfade" angewandt werden.

Das Uerfahren ist einerseits juristisch zu regeln, zum anderen sind Bewertungsmaßstabe CMeßgräßen, Indikatorend zu Einden, die die Umweltverträglichkeit bzw. -unverträglichkeit anzeigen. Der dritte, technische Aspekt betrifft die Definition und die Implementation der Methoden und Verfahren, mit Hilfe derer die UUP durcheührbar wird.

Die Informatik kann im letztgenannten Bereich Hilfestellung leisten, indem sie Instrumente zur Verfügung stellt, die einzelne Methoden oder eine Integration von Methoden sowie die notwendigen Daten zugänglich und komfortabel nutzbar machen. 


\section{DiE Unweltvertiägdichkeitsprüung}

Zunächst ist der Anwendungsbereich einer UUP einzugrenzen. Hierzu gibt es Richtlinien, die Beachtung finden müssen und es gibt pragmatische Aspekte. Die Bundesregierung hat 1975 "Grundsätze Eür die Prüfung der Umueltverträglichkeit öffentlicher Maßnahmen des Bundes" beschlossen, die den Anwendungsbereich sa breit wie möglich Fassen. Dies ist unter dem Aspekt einer ganzheitlichen Betrachtung der Umwelt sicher sinnvoll, praktisch können sich jedoch Prableme sowahl hinsichtlich der notwendigen Daten als auch hinsichtlich einer angemessenen Modellbildung ergeben, die eine engere Eingrenzung des Anwendungsbereichs geboten erscheinen lassen.

Die EG hat dazu eine Richtlinie /AMT 85/ erarbeitet, die von den Mitgliedstaaten bis zum Sommer 1988 übernommen werden muß. Die Richtlinie definiert zwei Typen von Vorhaben: solche, für die stets eine umfangreiche UUP durchgeführt werden salite, und solche, die nur einer vereinfachten Prüfung unterzogen werden müssen. Es ist jedoch davon auszugehen, daß die Richtiinie so allgemein gehalten ist, daß eine praktischen Ausgestaltung notwendig ist, und daß die Richtiinie auf Grund der Erfahrungan der Praxis weiterentwickelt wird.

Auch der Zeitpunkt einer UUP wird in der EG-Richtlinie definiert: eine UUP ist im Rahmen der Entscheidung über die Zulässigkeit eines Vorhabens durchzuführen. Dies ist sicherlich ein politischer Kompramiß, idealerweise muß die UUP bereits in der Planungsphase einsetzen, da hier eine Auswahl zwischen unterschiedlichen Alternativen getroffen wird. Weil sich in weiteren Projektphasen stets Veränderungen oder Kankretisierungen ergeben, ist es empfehlenswert, aufeinander aufbauende Teil-

Umweltverträglichkeitsprüfungen durchzuführen.

In der Bundesrepublik sind die umweltbezogenen Verwaltungskompetenzen auf viele vaneinander unabhängige Behörden verteilt, innerhalb derer zusätzlich hierarchische Strukturen in den Planungsprozeß eingehen. Grundsätzlich ist ein integrierter, koordinierender Planungsansatz, der alle Umweltaspekte in einem einzigen Verfahren erfaßt, notwendig, auch wenn eine Realisierbarkeit im Augenblick Eraguürdig erscheint. Ein möglicher Ansatz zur Realisierung sind auch hier aufeinander aufbauende TeilUmwel tverträglichkeitsprüfungen.

/AMT 85/

Amtsblatt der Europäischen Gemeinschaften (5.7.85) Richtlinie des Rates vam 27. Juni 1985 über die Umweltverträglichkeitsprüfung bei bestimmten öffentlichen und privaten Projekten (85/337/EWG) 
Die Verantwortung für die Durcheührung einer

Umweltverträglichkeitsprüfung soll nach verbreiteter Meinung (auch in der Richtlinie der EG) beim Verursacher, also beim

Projektträger 1iegen. Dies wird in den USA, die die UUP in der

Gesetzgebung verankert hat, beroits mit einigem Erfolg

praktiziert. Die genehmigenden Behörden haben bei dieser

Vorgehensweise nur noch eine Uberprüfung der vorliegenden UUP

(häufig wird nur eine Plausibilitatskontrolle möglich sein)

durchzuführen. Die Richtlinie der EG sieht eine

Zusammenarbeit mit den zuständigen Behörden vor, wenn der

Projektträger allein eine umfassende UUP nicht leisten kann. Wesentlich ist die Vollständigkeit und Durchschaubarkeit der UUP, die allein Uergleichbarkeit und Kantrolle ermöglichen.

Eine UUP ausschließlich von "Stellen mit besonderem UmweltSachverstand" durchführen 24 lassen, wird aus Mangel an qualifizierten Umweltplanern nicht möglich sein. Da jedoch in Zukunft ein erhöhter Bedarf an "Umweltverträglichkeits-Prüfern" zu erwarten ist, muß alles getan werden, um zumindest das vorhandene Defizit an Werkzeugen zu verringern. Solche Werkzeuge können, sind sie richtig konzipiert, die Durchführung der UuP erleichtern (betrifft Projektträger und Behörden), vergleichbar machen (Dokumentationsaspekt sowohl bzgl. der Methodik als auch bzgl. der Ergebnisse) und überprüfbar machen (durch unabhängige Stellen und die offentlichkeit).

Die UUP ist ein VerEahegn, mit dem Auswirkungen van Vorhaben auf die Umwelt konkret bewertet werden sollen. Zur Durchführung einer UUP gibt es Methoden, deren Angemessenheit vom konkreten Projekt abhängt. Ein entscheidungsunterstützendes System UUP (EUS UUP) ist ein Werkzeug für den Planer. Ansätze Für ein Konzept eines solchen Systems solien hier erläutert werden.

Die EG-Richtlinie definiert den Inhalt einer UUP. Diese Definition dient als Leitfaden Eür das Konzept und soll deshalb kurz erläutert werden:

Eine UUP macht Angaben Über die Ausuirkungen eines Vorhabens auf die natürlichen Ressourcen (Wasser, Boden, Luft, Klima, Flara und Fauna) sowie ihre Wechselwirkungen) und auf die vom Menschen gestaltete Umwelt (Architektur und Landschaft).

Der Projektträger hat den zuständigen Behörden Folgendes varzulegen:

$1)$ eine Beschreibung des Projekts (physische Eigenschaften des Uorhabens, Bedarf an Grund und Boden, Beschreibung des Produktionsprozesses, zu erwartende Emissionen und zu erwartender AbFal13, moglichst mit alternativen Ausprägungen

2) eine Beschreibung der beeinflußten Umwelt (gegebenenfalls grenzüberschreitend) 
3) eine Beschreibung der Auswirkungen durch

- den Baukírper ader die Infrastruktur

- den Verbrauch von Umuelt-Ressourcen

- Emissionen/Ner- und Entsorgung der Projektanlage

4) eine Wirkungsanalyse (Beschreibung der verwendeten Methoden)

5) eine Beschreibung der Maßnahmen, mit denen die in der Wirkungsanalyse Festgestellten Auswirkungen auf die Umwelt vermieden, verringert oder kompensiert werden soll

6) eine Beschreibung der bestehenden Umweltschutz- und Raumordungspläne des beainflußten Gebiets, die für das Uorhaben von Bedeutung sind

7) Ealls Für das Uorhaben in der Wirkungsanalyse erhebliche Auswirkungen Festgestelit wurden, eine Erläuterung der Wahl von Standort und Ausführung des Uarhabens, insbesandere im Vergleich zu mäglichen Alternativen

B) abschließend eine nichttechnische Zusammenfassung aller Angaben

Notwendig für eine UUP sind jedoch heute noch nicht eindeutig definierte Kriterien zur Beurteilung der Umueltverträglichkeit, d.h. Maßstäbe zur Beschreibung von "Umweltqualität".

Auch über die anzuwendende Methodik gibt es Differenzen, da die Praxis der UUP noch nicht institutionalisiert ist. Es bietet sich dem Informatiker hier die Chance, zusammen mit dem Anwender, für ein neues Gebiet einen Methodenbaukasten zu entwickeln, der eine der UUP des jeweiligen Prajekts angemessene Methodenzusammenstellung arlaubt /PIE B6/.

Um diese Angemessenheit der Methodenzusammenstellung, für die die Uerantwortung selbstverständich weiterhin beim Anwender bleibt, zu ermüglichen, muß das Werkzeug EUS UUP auf einer geeigneten

Architektur aufbauen. Hier sollen die Möglichkeiten der Künstlichen Intelligenz, insbesondere der Wissensbasierten Systeme oder Expertensysteme, auf ihre Anwendbarkeit in einem Entscheidungsunterstützenden System untersucht werden.

TPIE $96 /$

Pietsch, J. 1986

Umweltverträglichkeitsprüfung in der kommunalen Planung Maßstabe und Methodenbausteine

Uortrag auf der 3. Fachtagung: "Planung Für den Umweltschutz und die Rolle der Umweltverträglichkeitsprüfung" 10. - 12. Màrz 1986, München. 


\section{Uberlegungen zu Einsatz won Expertensygterelenenten in einer entscheidungsunterstützenden Syster uUP}

Expertensysteme sind ein Produkt der Forschung des Wissenschaftsgebietes "Künstliche Intelligenz". Diese Systeme haben die ersten Schritte des Weges heraus aus den Forschungslabors hin zu erfolgreichen Anwendungen bereits hinter sich. Die Konzepte, die Expertensystemen zugrunde liegen, lassen sich direkt oder modifiziert verwenden, um Teile eines Systems zur Unterstützung der Umweltverträglichkeitsprüfung zu entwickeln.

Die Anwendbarkeit der Komponenten von Expertensystemen in einem entscheidungsunterstützenden System UUP soll untersucht werden:

- Wissensbasis

- Inferenzkamponente

- Erklärungskampanente.

Zunächst soll jeweils kurz das Verständnis der Autorin erläutert werden, um auf Grund dieser Sicht Anwendbarkeit, Lokalisierung der Anwendbarkeit und Notwendigkeit zur Modifikation oder Erweiterung zu diskutieren.

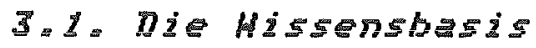 \\ 3.2.I HIS5ensonsteILung}

Man kann zwischen deklaratiber und proreduraler Histengdargteliung unterscheiden. Das Wissen um Fakten wird deklarativ, das Wissen um deren Verwendung prozedural dargestelit. In rein deklarativen Darstellungsformen ist das Wissen über Verwendung und Erzeugung in allgemein verwendbaren Programmen, die die deklarative Wissensbasis manipulieren, zusammengefaßt, wahrend in prozeduraler Darstellungsfarm Wissen über die Anwendung und die Erzeugung von Wissen explizit dargestellt wird. Während die Protagonisten der deklarativen Darstellungsform den Aspekt der Repräsentation in passenden Formalismen für am wichtigsten halten, argumentieren die Prozeduralisten, daß das nutzbare Wissen eines Bereiches intrinsisch mit dem speziellen Wissen über die Benutzung dieses Wissens verbunden ist.

Beispiel für eine rein deklarative Darstellungsform ist die Prädikatenlogik, Realisierungen des prozeduralen Schemas stellen die ACTOR-Sprachen dar.

Beide Darstellungsformen haben Nachteile. Die Irennung zwischen der Beschreibung der Elemente der Welt und ihrer Erzeugung und Nutzung erzeugt Konsistenzprobleme, ist häufig unangemessen und verhindert die Kammunizierbarkeit des mit Hilfe der Darstellung implementierten Modelis. 
Eine objektorientierte Darstellung überwindet die Trennung durch Integration beider Aspekte. Ein bbjekt wird bezüglich der problembedingten Abstraktion vollständig beschrieben, d.h. sowohl hinsichtlich seiner Beschreibung als auch hinsichtlich Erzeugung, Ueränderung und Aktionsfähigkeit.

"Objekt: allgemeiner Gegenstand des Interesses, insbesandere einer Beobachtung, Untersuchung, Messung"

Eine objektorientierte Darstellungsform ist von M. Minsky/MIN 75/ unter dem Namen Frame Eür die KI entwickelt worden. Die bekanntesten Repräsentationssprachen, die dieses Konzept implementieren sind FRL und KRL. CFür eine detailliertere Beschreibung der Darsteliungsmethode wird auf /SCH B6/ verwiesen)

\subsubsection{DiE Hissensbesis in eine EUS UUP}

Für das zu entwickelnde entscheidungsunterstützende System unterstellen wir ein objektorientiertes Architektur-Modell. Dbjekte sind die Elemente der Umwelt und die im System implementierten Methoden für die Prüfung der Umwel tverträglichkeit.

Konkret ist für jedes in das System zu integrierende Dbjekt zunächst seine stellung innerhalb der bereits vorhandenen Objekthierarchie $z u$ definieren. Wir gehen van einem evalutianären System aus, das sich durch seine Nutzung verändert und dessen Labenszyklus in erster Linie durch "Wachsen und Verändern" beschrieben werden kann. Die Definition des dbjekts beeinhaltet die Eigenschaften und die Leistungen des abjekts, damit sind sowahl das objekt selber als auch seine Schnittstellen zu anderen objekten beschrieben.

TMIN 75/

Minsky,M.L. 1975

A Framework Far Representing Knouledge

in:

Winston, P. (Ed.) 1975

The Psychology of Computer Uision

MoGraw-Hi11, New York.

\section{/SCH B6/}

Schwabl, A. 1986

Diskussion einer Darstellungsmethodik aines

entscheidungsunterstützenden Systems für den Umweltbereich in:

Paga, B. 1986 (Hrsg.)

Informatik im Umweltschutz

Anwendungen und Perspektiven

Qldenbourg Verlag, München. 
Dieser objektorientierte Ansatz bietet dem Benutzer des Systems eine homogene Sicht, die sowohl Transparenz und Verständichkeit des existierenden Systems ermäglichen, als auch seine Schnittstellen zu anderen Qbjekten beschrieben.

Dieser Ansatz soll an einem Beispiel erläutert werden:

Ein Qbjekt der Umwelt ist ein Fluß. Dieser wird beschrieben durch seine geographische Lage, durch seine Strömungsrichtung, seine Strömungsgeschwindigkeit, seine Inhaltsstoffe, die in ihm lebende Flora und Fauna, seinen UEerbewuchs und möglicherweise weitere Attribute.

Das abstrakte Objekt Fluß beschreibt also alle Eigenschaften, die auf alle Flüsse zutreffen. Eine Spezialisierung des objekts Fluß ist das Objekt Gezeitenstrom, das sich in bestimmten Attiibuten vam aligemeineren objekt Fluß unterscheidet.

Das abstrakte Objekt Gezeitenstrom nimmt im konkreten Fluß "Elbe" Gestalt an. Es Findet eine weitere Spezialisierung statt.

Will man das Objekt Elbe beschreiben, bedient man sich des abstrakten Dbjekts Fluß und seiner Spezialisiarung Gezeitenstrom und gibt den dort definierten Attributen werte, Eügt gegebenenfalis weitere Attribute hinzu oder streicht andere Attribute, abhängig davon, ob dieser Fluß Besonderheiten hat, die ihn von dem beschriebenen abstrakten Fluß und dem abstrakten Gezeitenstrom unterscheiden.

Oben wurde eine statische Beschreibung eines Flusses gegeben. Soll die Dynamik eines Flusses definiert werden, z.B. der Transport von Schwebstoffen in diesem Fluß, so benótigt man dazu die Implementation der entsprechenden

Iransportgleichungen, die sich die bereits vorhandene topologische Beschreibung des Flusses zunutze machen kónnen. Weitere prozedurale Elemente der Beschreibung des Objekts Elbe können im Bedarfsfall hinzugefügt werden: Falls etwa eine Begradigung des Flusses geplant sein solite, wäre oine Berechnung der Veränderung der Strb̈mungsgeschwindigkeit und der sich durch die Tide ergebenden Wasserhöhe notwendig. Die zur Berechnung notwendigen Strómungsgleichungen sind zu implementieren.

Eines der Grundprinzipien in einem objektorientierten System ist die Vererbung. Es gibt i.w. zwei Uerwandtschaftbeziehungen zwischen den Dbjekten: die Element-Beziehung (Beziehung zwischen einer Konkretisierung und einem abstrakten abjekt, auch Element und Klasse genannt, z.B. Elbe-Fluß) und die Teilklasse-Beziehung (Beziehung zwischen Klasse und Teilklasse, z.B. FlußGezeitenstram). Ein Dbjekt hat aufgrund seiner Stellung in der 
Hierarchie nicht nur seine explizit definierten Eigenschaften, sondern erbt, d.h. übernimmt alle Eigenschaften der hierarchisch höheren Dbjekte, sofern diese Eigenschaften nicht in der Definition des objekts unterdrückt oder überschrieben werden. Dieses Vererbungsprinzip solite man nun nutzen, wenn man in einem EUS UUP eine Methode Eür die Berechnung van Wasserströmungen implementieren will, indem man etwa ein abstraktes Modeli "Strömungsgleichungen" und als Elemente davon Instanzen dieses objekts, die die unterschiedlichen mathematischen Lüsungswege implementieren, definiert.

Wenn man solche Berechnungsmethoden im Dbjekt Elbe implementieren würde, wären sie nur an dieser Stelle nutzbar, ein abjekt Rhein, das hierarchisch auf derselben Ebene angesiedelt wäre, hätte keinen Zugriff. Desweiteren ist eine klare Trennung zwischen den Objekten der Umwelt und den Methoden, mit denen Umweltszenarien erarbeitet und durchgespielt werden kónnen, anzustreben.

Die Stellung des Objekts Strömungsgleichungen innerhalb der Hierarchie sollte sich an den Zugriffsbedürfnissen der übrigen Dbjekte des EUS UUP orientieren.

Die besonderen Eigenschaften eines objektorientierten Systems, die es für ein EUS UUP vorteilhaft erscheinen lassen, sind

- Verständichkeit (der Systemstruktur)

- Uberschaubarkeit (Objekte sind durch ihre Funktion und ihre Schnittstelien bescheieben)

- Modularität ca.h. insbesondere Unabhängigkeit seiner Komponenten]

\subsection{Die Problewbehand ungs-Konponente}

\subsection{Inferenzkoponente und Kontrollstrategie}

Die Problembehandlungs-Komponente bearbeitet die vom Benutzer des Systems definierte Prablemstellung und versucht, eine Lösung entweder direkt in der Wissensbasis $2 U$ Einden oder sia abzuleiten, d.h. aus den zur Verfügung stehenden Fakten Schlüsse zu ziehen (Inferenzen): Expertansysteme arbeiten nach einer Zielvorgabe und vorgegebenen Randbedingungen, jedoch ohne vom Programmierar vargeschriebenen Weg.

Die explizite Irennung des "Wissens" in der Wissensbasis von der Uerwendung dieses Wissens zeichnet Expertensysteme aus.

Die KI versteht unter Wissen das Wissen über ain Spezialgebiet, sogen. Expertenuissen, das man am besten mit dem Begriff Erfahrungswissen ader Heuristiken beschreiben kann; und schließt Allgemeinwissen vollständig aus. 
Es handelt sich damit um die Suche nach einer optimalen ader zumindest nach einer gültigen Lüsung. "Problemlösen", d.h. Manipulation von Wissen, und Suchstrategien sind charakteristische Methoden der Künstlichen Intelligenz. Der Begriff "Problemlösen" beschreibt ein Eormales Vorgeher, er entspricht nicht dem menschlichen Uargehen, die zu läsenden "Probleme" müssen präzise Formulierbar sein.

Um den Suchaufwand zu reduzieren, versucht man, aufgabenspezifisches Erfahrungswissen (Heuristiken) in den Problemlüsungs-Prozeß zu integrieren. Man spricht in diesem Zusammenhang von einem heuristischen Ansatz und setzt diesen in Gegensatz zum algorithmischen Ansatz. Diese sogen. intelligente Suche hat Minsky 1966 so beschrieben:

"What makes for intelligent behaviour is the collection of methods and techniques that select, what is to teied next, that size up the situation and choose a plausible (if not always good) move and use information gathered in previous attempts to steer subsequent analysis in better directions." /MIN 66/

Auch die heuristische Uorgehensweise muß algorithmisch formulierbar sein, "es handelt sich lediglich um eine Klasse größerer AlIgemeinheit" (/Sche BG/)

Es lassen sich zwei wesentliche Elemente der Prablembehandlungs-Komponente extrahieren:

- der Inferenzmechanismus, mit Hilfe dessen neues wissen aus der Wissensbasis abgeleitet werden kann (Schlußfalgern).

- Ein einfaches Beispiel Eür eine Inferenzregel, die auf eine Logik-Darstellung anwendbar ist, ist der Modus Panens, der besagt, daß, Falis ' $a$ ' und 'aus a Folgt b' wahr, ist auch ' $b$ ' wahr ist (Formale Inferenz).

- Bei prozeduraler Darstellung ergibt sich die berechenbare oder prozedurale Inferenz: eine Prozedur, die ein Modell des die Problemstellung oder Teilproblemsteliung betreffenden Bereichs darstellt, wird ausgeführt und liefert als Ergebnis eine Problemlásung oder eine Teilprablemlüsung.

\section{/MIN 66/}

Minsky,M.L. 1966

Artificial. Intelligence,

Freeman, San Francisco, London.

\section{/SCHE BE/}

Schefe, P. 1986

Künstliche Intelligenz- Uberblick und Grundlagen

Bibliographisches Institut, Mannheim, Wien, Zürich. 
- Meta-Inferenz macht sich das Wissen um das in der Wissensbasis vorhandene Wissen zunutze, um neues Wissen abzuleiten.

- Die Kontrallinformation, die die Such-Strategie definiert.

Man unterscheidet i.w. zwei grundlegende Strategien:

- die Vorwäts-Verkettung, bei der ausgehend von gegebenen Fakten mit Hilfe der Inferenzregeln oder durch Anwendung des explizit vorhandenen Wissens neue Fakten abgeleitet werden, bis das gewünschte Ergebnis erreicht ist, und

- die Rückwärts-Verkettung, bei der ausgehend von Zielen versucht wird, eine Reihe von Regelanwendungen $z u$ finden, die die Ausgangssituation in die Zielsituation überEühren, d.h. ausgehend von einer Hypathesenmenge wird versucht, eing Hupothese zu verifizieren.

Die Wahl zwischen Vorwärts- und Rückwärts-Uerkettung sollte van der Art der in der Wissensbasis repräsentierten Regeln gewählt werden. Falls von jedem Knoten im Suchgraph nur wenige andere Knoten erreichbar sind (small fan out) andererseits jedoch viele Regeln auf denselben Knaten führen (large fan in), ist in aller Regel Vorwarts-Verkettung effizienter, sind die Verhältnisse umgekehrt, sol1te Rückwärts-Verkettung gewählt werden.

Kontrallinformationen lassen sich etwa als Meta-Regeln detaillieren, d.h. als Regeln über die Reihenfolge der Anwendung von Regeln ader die Bedingungen, unter denen bestimmte Wissensinhalte anwendbar werden.

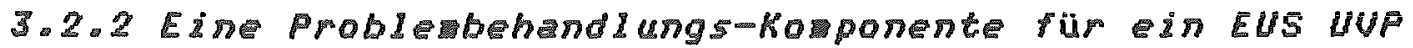

Die Kontrall-strategie in einem EUS UUP muß sich an den oben beschriebenen Vorgaben der EG Eür eine UmweltverträglichkeitsPrüfung orientieren. Hier soll dies an der Eingrenzung des Anwendungsbereichs der UUP gezeigt werden. Dieser Teilbereich der UUP läßt sich gut als Expertensystem realisieren. Es handelt sich hierbei um eine "Bestimmungsaufgabe". ․h., ausgehend von einer Befragung des Umweltprüfers bzgl. des zu prüfenden Projekts versucht das System aus seiner Wissensbasis die beeinflußten Umweltbereiche abzuleiten. Das für diesen Teilbereich notwendige Expertenuissen ist regelorientiert und sollte deshalb auch so dargestellt werden. Es Ealgt daraus eine Segmentierung des Gesamtprablems UUP in Teilprableme, die sich aus oben aufgeführten sieben den zuständigen Behörden vorzulggenden Beschreibungen bzw. Analysen ergeben.

Das lbjekt "Bestimmung des beeinflußten Umweltbereichs" kann intern ohne Verletzung des definierten objektorientierten Modells regelbasiert sein. Das objekt enthält in der Regelbasis das 
objektspezifische Wissen und Regeln zur Fragenauswahl und zum Ausschluß von Umweltbereichen, d.h. die Wissensbasis und die Problembehandlungs-Komponente. Die Uermutung 1 iegt nahe, daß sich die Eingriffe in die Umwelt grob- und nachfalgend Feinklassifizieren lassen, so daß sich eine Bestimmung des beeinflußten Umwel tbereichs effizient hierarchisch durchführen läßt. Die Literatur gibt einige Beispiele für den Einsatz von Expertensystemen zur Bestimmung von bbjekten und beschreibt dil übergeordnete Kontrolistragie als Uorwärtsverkettung.

Das Ergebnis der Suche sowie die Suche selbst lassen sich zunächst für die Hersteliung einer "Beschreibung der beeinflußten Umwelt" verwenden ( $s$. Punkt 2 der UUP), desweiteren dient es als Eingangsgröße für die Wirkungsanalyse (Punkt 3). Zudem läßt sich aus dern Ergebnis der Befragung eine Beschreibung des Projekts ableiten (Punkt 1 ).

Der fünfte Punkt im Leistungskatalog einer UUP, eine Beschreibung der bestehenden. Umueltschutz- und Raumordnungspläne des beeinflußten Gebiets, die für das Uorhaben von Bedeutung sind, läßt sich mit den gleichen Methoden wie die Eingrenzung des Anwendungsbereichs realisieren.

\subsection{Die Erklärungskoponente}

\subsection{Technik und Hethoden rür Erklärungskovponenten}

Eine Erklärungskomponente hat die Aufgabe, eine für den Benutzer verständliche und dem jeweiligen Arbeitsfortschritt angemessene Erklärung als Antwort auf eine Frage zu erzeugen. Damit wird eine größere Transparenz und Kontrallierbarkeit, sowie eine bessere Handhabung des Systems erreicht, die zu höherer Akzeptanz und wohl auch höherer Produktivität führen:

"such programs are more convincing when right, and easier to debug when wrong" /STS 77/

Erklärungskomponenten lassen sich bzgl. der struktur der Wissensbasis, bzgl. ihrer Mächtigkeit und bzgl.der Benutzeroberfläche der Erklärungskomponente klassifizieren:

\section{/STS 77/}

Stallman, R.M. Sussman,G.J. 1977

Forward reasoning and dependency-directed backtracking in a system for computer-aided circuit analysis in:

Artificial Intelligence, 9, 5. $135-196$. 
- so lassen sich Ableitungen regelgesteuerter Inferenzsysteme relativ verständich erklären, Resultate einer Prozedur können dagegen nicht ohne weiteres benutzeradäquat nachvollzogen werden;

- die Mächtigkeit einer Erklärungkomponente drückt sich in den beantwortbaren Fragenbereichen aus: Erklärung einer Tatsache oder Behauptung, Begründung einer Frage oder einer Aufforderung des Systems;

- die Generierung von Erklärungen geht gewbhnlich von einer schriftlichen, natürlichsprachlichen form aus, im Anwendungsbereich Umweltplanung erscheinen auch graphische Hilfsmittel sinnvall.

Von wesentlicher Bedeutung ist auch die Differentierung des Erklärungsbegriffs:

- Erklärung von Tatsachen, Behauptungen, Ereignissen und Prozessen.

Beispiel: Erklärung, warum über dem Gebiet von Billstedt mit erhohtem Dioxin-Niederschlag zu rechnen ist.

- Erklärung des Zusammenwirkens eines komplexen Gebildes. Beispiel: Zusammenuirken der belebten und unbelebten Bestandteile eines Biotops.

- Erklärung von Begriffen.

Beispiel: Erklärung von Begriffen aus dem Umweltschutz- und Raumordnungsracht.

Für den Informatiker als Entwickler eines Expertensystems mit einer Erklärungskomponente ist klar, daß es Eür jedes Werhalten des Systems prinzipiell eine Kausalerklärung gibt. Dies schließt aber keineswegs aus, daß es Anwendungssituationen geben kann, in denen eine benutzeradäquate Erklärung nicht generiert werden kann. Grundsatzlich lassen sich verständliche Antworten nur generieren, wenn das Expertensystem seine Ableitung auf Grund eines dem Benutzer verständlichen Modells durchführt. Wenn also das Verfahren, nach dem das system vorgeht, ein benutzeradäquates ist.

Beispiel:

Wenn ein Simulationsmodel1 sich einer anderen Berechnungsmethode bedient, als sie der Anwender gewthnlich ( $z . B$. bei "von Hand"-Rechnungen benutzt), weil diese Methode effizienter ist (etwa FourierTransformationen), kann das Modell nicht automatisch verständlich erklärt werden. Eine Erklärungskomponente ist jedoch nur dann sinnvoll, wenn sia in der lage ist, Benutzermodell-adäquate Erklärungen zu generieren. 


\subsubsection{Die Erklärungskomponente rür ein EUs uv}

Hier soll insbesandere die Erklärungskamponente für das regelorientierte Expertensystem zur Eingrenzung des Anwendungsbereichs der UUP konzipiert werden. Diese Erklärungskomponente erfülit zwei Aurgaben

- Sie macht für den Benutzer die Ableitungen des Systems durchschaubar und damit nachprüfbar

- Sie ist gleichzeitig eine adäquate Aufbergitung der "Beschreibung der beeinflußten Umuelt" und der "Beschreibung des Projekts"

Die Struktur der der Erklärungskamponente zugrundeliegenden Wissensbasis ist regelorientiert. Aus dieser Darstellungsform läßt sich recht einfach eine verbale, benutzeradäquate Erklärung ableiten.

Die zu beantwortenden Fragen lassen sich in zwei Kategorien einordnen:

- Begründung der zu prüfenden Umweltbereiche durch Erklärung 1hres Zusammenwirkens

- Begründung der gesetzlichen Notwendigkeit bestimmter Prüfungen.

Mithilfe dieser Erklärungsklassen läßt sich ein Ergebnis ebenso erklären, wie sich eine Anfrage oder eine Aufforderung des Systems motivieren läßt.

Die Generierung der Erklärungen sollte sowohl am Bildschirm für den interaktiven Gebrauch zur Verfügung stehen, als auch für die Ergebnisaufbereitung (etwa für die genehmigende Behörde) druckbar bzw. plotbar sein.

Die Begründung der zu prüfenden Umweltbereiche durch Erklärung ihres Zusammenwirkens sollte nicht in rein verbaler Form gegeben werden. Eine graphische Aufbereitung dieser Zusammenhänge bietet sich an, da sowohl die technischen Uaraussetzungen für eine graphische Benutzerschnittstelle in der Interaktion (GraphikBildschirm, Fenstersysteme) als auch die Programmiertechniken für diese Art der Mensch-Maschine-Kommunikation entwickelt sind. Die bei der graphischen Darstellung komplexerer Zusammenhänge auftretenden Probleme der Unübersichtlichkeit lassen sich mithilfe der Fenstertechnik recht gut behersschen: ausgehend von groben Zusammenhängen können durch Zoomen in Eeinere Ebenen und durch uberlagern von Fenstern verschiedener DetaillierungsstuFen Zusammenhänge sichtbar gemacht werden.

Graphikdarstellungen liegen zudem im Umweltbereich sehr nahe, sie sind sicherlich die natürlichste form der Darstellung. 


\section{Konzept für ein regelorientiertes Expertensyster zur Unterstutzung der Eingrenzung des Anwendungsbereichs der uUp}

Das Expertensystem zur Unterstützung der Eingrenzung der UUP basiert auf einer regelorientierten Wissensbasis. Die verwendete Kontrollstragie ist Uorwärtsverkettung. Die Wissensbasis ist hierarchisch organisiert. Diese Struktur ergibt sich sowohl bzgl. der Projekte als auch bzgl. der Umwelt zwangsläufig.

Nach einer Selbsteinführung des Systems, in der der Anwender in die Benutzung des Systems aingeführt wird, werden durch Ubersichtsfragen die Basisfakten, die im System für die Ableitungen nötig sind, vam Benutzer eingegeben. Hiermit wird die dynamische Wissensbasis, die den aktuellen Kenntnisstand des Systems repräsentiert, aufgebaut.

Das Expertensystem orientiert sich am Ablauf der UUP: die Projektdefinition, die eine räumliche, zeitliche und sachliche Beschreibung der Maßnahme widergibt, wird vom Benutzer in einem durch das System geführten Dialog eingegeben.

Das System versucht dann auf der Grundlage der vorhandenen Regeln durch SchlußEalgerungen über bekannte, vom Benutzer bereits erfragte oder noch zu erfragende Fakten ein Ergebnis abzuleiten.

Für solche Ableitungen sind mindestens 3 Regeltypen notwendig:

w Steuerregeln, die die Reihenfalge des Vorgehens festlegen Z.B. wenn es sich bei dem Projekt um ain Kraftwerk handelt, frage als nächstes nach dem Typ des Kraftwerks

Kansistenzregein, die ein allgemeines Modell des Bereichs abbilden, das in vielen Fällen banal erscheint, aber zur Lösung der Aufgabe notwendig ist

Z.B.

wenn das eingeleitete Kühlwasser nicht zu warm ist, aber die Iemperatur ist nicht in Ordnung, dann ist das eingoleitete Kühlwasser zu kalt

* Eingrenzungsregeln

stellen ein spezielles Modell dar, das die Eingrenzung ermüglicht

Z.B.

wenn Flußwasser zur Kühlung verwendet wird, besteht ein Yerdacht auf Einleitung erwärmten Wassers und die Erwärmung des Flußwassers ist in die Umweltverträglichkeitsprüfung einzubeziehen. 
Die Erklärungskomponente kann zu jedem Zeitpunkt auf eine "WarumFrage" des Benutzers die vom System geforderten Eingaben begründen. Der Kontext der aktuell gestellten Frage ergibt sich aus den bisher angewandten Regeln zusammen mit den daraus sich ergebenden Eeuerbereiten Regeln. Die Erklärungskomponento ermüglicht ebenso "Wie-Fragen": wie hat das System einen Umweltbereich abgeleitet? Der Zustand der dynamischen Datenbasis kann aufbereitet ausgegeben werden, um den aktuellen wissensstand des Systems zu erklären.

Die Erklärungskomponente muß auf geeignete Ausgabeformen zugreifen können:

- verschiedene Möglichkeiten, Regeln problemadäquat ausgeben zu können

- die Ergebnisse der angewandten Regeln verbal ausgeben zu können

- die untersuchten oder gefundenen(vom System bewiesenen) Zusammenhänge graphisch darstellen zu künnen. Diese Darsteliung kann unterstützt werden durch das Hervorheben der gerade untersuchten und als verdachtig erkannten Umweltbereiche z.B. durch "Blinken".

Auf Grund der durch den Benutzer eingegebenen Projekt-Beschreibung erstellt das Expertensystem eine Umwelterklärung, die aus der Beschreibung der potentiell betroffenen Umwelt und der Beschreibung der Auswirkungen auf die Umwelt besteht.

Die Benutzerschnittstelle macht von den Möglichkeiten der Graphik Gebrauch. Durch die hierarchische Drganisation des WissensBereiches ist es möglich, die Komplexität, die Graphiken unpraktikabel macht, zu beherrschen: durch Zoomen kann zwischen den Hierarchiestufen gewechselt werden, durch Verwendung von Bildschirmfenstern können Ausschnitte verschiedener Hierarchieebenen gleichzeitig am Bildschirm sichtbar gemacht werden.

Die Zusammenhänge zwischen der Maßnahme und der durch sie betroffenen Umwelt läßt sich graphisch durch ein Netz darstellen, mithilfe etwa von Balkengraphik lassen sich der Zustand der betroffenen Umwelt in Beziehung zu Grenzwerten bzw. anzustrebenden werten setzen.

Die Ergebnisausgabe und die Erklärungskomponente benutzen diese graphischen Möglichkeiten: am Bildschirm wird wie oben beschrieben vorgegangen, die Präsentation der Ergebnisse erfalgt ebenfalls graphisch unterstützt über Druck und Plot.

Eingabeinstrument solite; soweit es nicht texturlie Eingabe betrifft, nicht die Tastatur sein. Geeignetes Eingabeinstrument scheint die Maus zu sein, mit deren HilEg man am Bildschirm eine Menüauswahl treffen oder Aktivierungen durchführen kann. 
Das Expertensystem "Eingrenzung UUP" ist Teil des EUS UUP, das u.a. ein Simulatianssystem für Wirkungsanalysen enthält. Für dia Ausgabe von Simulationsergebnissen ist es wünschensuert, die Graphikfahigkeit des Systems zu erweitern: bewegte Graphik oder Animation gibt den Darstellungsmöglichkeiten des Systems aina neue Qualitä.

Das Expertensystem "Eingrenzung Uup" wird in Zusammenarbeit mit kampetenten Anwendern erstellt und erprabt, um erstmalig ein Planungsinstrument für private und behördliche Projektträger prototypisch zur Verfügung stellen zu kb̈nnen und ErFahrungen für das Gesamtsystem EUS UUP zL sammein. 


\section{Literatur}

\section{/Amt 85/}

Amtsblatt der Europäischen Gemeinschaften (5.7.85)

Richtlinie des Rates vom 27. Juni 1985 über die

Umweltverträglichkeitsprüfung bei bestimmten dffentichen und privaten Projekten (85/337/EWG)

\section{Min 65/}

Minsky,M.L. 1966

Artificial Intelligence,

Freeman, San Francisco, Landon.

/Pie 86/

Pietsch, J. 1986

Umweltverträglichkeitsprürung in der kommunalen Planung Maßstabe und Methodenbausteine

Uortrag auf der 3. Fachtagung: "Planung Für den Umweltschutz und die Rolle der Umueltverträglichkeitsprüfung"

10. - 12. Márz, München.

\section{/Sche 86/}

Schefe, P. 1986

Künstliche Intelligenz- Uberblick und Grundlagen

Bibliographisches Institut, Mannheim, Wien, Zürich.

/Sohw $86 /$

Schwabl, A. 1986

Diskussion einer Darstel Iungsmethodik eines

entscheidungsunterstützenden Systems für den Umweltbereich

in:

Page, B. 1986 (Hrsg.)

Informatik im Umweltschutz

Anwendungen und Perspektiven

oldenbourg Verlag, München.

Stallman,R.M. Sussman, G.J. 1977

Forward reasoning and dependency-directed backtracking in a system for computer-aided circuit analysis

in:

Artificial Intelligence, 9, S. 135 - 195. 
KONZEPTE ZUR UNTERSTUTZUNG DER MODELLBILDUNG UND SIMULATION am Beispiel der Modelibildung auf dem Umweltsektor

L. Hilty

Universitat Hamburg

Fachbereich Informatik

\section{Zusammenfassung}

Die Entwicklung Formaler Modelie, ihre Implementation auf dem Computer und die Durcheürung von Simulationsexperimenten gehören zum methodischen Instrumentarium zahlreicher Fachgebiete. Besonders auf dem Umweltsektor wird der Computer zunehmend als Medium für die Modelibildung genutzt. Die Freiheitsgrade, die dieses Medium prinzipiell zuläßt, werden jedoch - u.a. aufgrund des hohen Implementationsaufwandes für Computermodelle - nur zu einem geringen Tail ausgeschöpt. In der Informatik wurden daher verschiedene Konzepte zur Unterstützung der Entuicklung, Implementation und Anwendung yon Modelien entwickelt (Simulationssprachen, Simulationssysteme, Modellbanksysteme).

Nach der Klärung einiger Grundbegriffe und der Klassifikation van Modelien werden diese Konzepte kurz beschrieben und voneinander abgegrenzt. Es werden einige Anforderungen an Modelibildungs - und Simulatianswerkzeuge Earmuliert, die van den existiaranden Systemen leider nur teilweise erfüllt warden. Möglichkeiten der Verbindung herkümmlicher Simulationstechniken mit Techniken aus dem Bereich der Künstlichen Intelligenz werden aufgezeigt, und einige grundsätzliche Uberlegungen zur Rolle der Simulation auf dem Umweltsektor werden wiedergegeben.

\section{Gliederung}

1. Einleitung

2. Begriffskiärung

3. Klassifikation van Madellen

4. Konzepte zur Unterstützung der Modellbildung und simulation

4.1 Simulationssprachen

4.2 Interaktive Simulationssysteme

4.3 Model lbanksysteme

4.4 Anforderungen an Modelibildungs-/Simulationswerkzeuge

5. Simulation und Künstliche Intelligenz

6. Chancen und Risiken der Simulation auf dem Umweltsektor

7. SchlußFolgerung 


\section{Einleitung}

Modelle spielen in den meisten Bereichen von wissenschaft und Iechnik eine zentrale Rolle. In zahlreichen Fachgebieten existiert eine längere methodische Tradition der Entwicklung und Anwendung von Modelien, die meist an einen relativ eingeschränkten Madel 1 begriff gebunden ist. So wird etwa ein Biologe etwas anderes unter einem Modell verstehen als ein Meteorologe; ein Wirtschaftswissenschaftler wird von einem anderen Modellbegriff ausgehen als ein Psychologe.

Die Möglichkeit, Formale Model le auf einem digitalen Rechner zu implementieren, hat zusätzliche Freiheitsgrade für die Modellbildung geschaffen. So künnen beispielsweise umfangreichere Modelle entwickelt werden, da der Rechenaufwand eine immer geringere Rolle spielt, und es können neue Modelitupen insbesondere Simulationsmodelle - eingesetzt werden, wo man früher auf analytische Modelle angewiesen war. Es besteht Ferner die Möglichkeit der Kopplung van bestehenden Modellen mit anderen Modelien oder mit umfangreichen Datenbeständen. Nicht zuletzt bietet der Computer die Chance, interdisziplinäre Modelistudien durchzuführen, bei donen das gemeinsam entwickelte Modell auch als Kommunikationsmedium für die beteiligten wissenschaftler dient.

Formale Modelle werden letztlich mit dem ziel entwickelt, Komplexitat 24 beherrschen. Man würde daher erwarten, daß bei der computergestützten Modelibildung und Simulation alle Register gezogen werden, welche die. Informatik zur Bewältigung von Komplexität anbietet. Dies würde $u . a$. die Verwendung strukturierter Implementationssprachen, die Modularisierung von Modelien, die Anwendung der Computergrafik zur Visualisierung von Modelistruktur und -verhalten souie eine interaktive Arbeitsweise nahelegen.

Diese Möglichkeiten werden jedoch in der Praxis nur teilweise genutzt - was hauptsächlich darauf zurückzuführen ist, daß häufig inadäquate Werkzeuge für die Modellbildung und Simulation eingesetzt werden. Ein adäquates Werkzeug sollte insbesondere sicherstellen,

- daß nicht der Implementationsaufwand, sondern das Wissen des Modellentwicklers die Grenzen der Modelibildung bestimmen;

- daß das erstellte Programm leichter zu durchschauen und zu beherrschen ist als das Realsystem, das es modellieren sall;

- daß ein implementiertes Model1 nicht nur vom Modellentwickler selbst benutzt werden kann, sondern auch anderen Persanen zur Anwendung und kritischen Begutachtung zur Verfügung steht;

- daß die Modelistruktur sowie die im Modell enthaltenen Annahmen klar dokumentiert sind. 
Diese Forderungen sind in der Praxis leider keineswegs selbstverstandlich - das gilt insbesondere im Zusammenhang mit allgemeinen höheran Programmiersprachen wie $\mathbb{Z} . B$. FORTRAN, dia häufig zUr Madallimplementation verwendet werden.

Diaser Beitrag versucht einige wege zur Unterstützung der Modelibildung und Simulation durch adäquate Softwarekonzepte aufzuzeigen. Aufgrund der uneinheitlichen Ierminologie auf diesem Gebiet wird der Begriffsklärung und der Klassifikation von Modellen relativ breiter Raum gegeben.

\section{Beqriffsklärung}

\section{Mode 11:}

Der Begriff des Modells wird in der Fachliteratur sehr unterschiedlich definiert. Es ist in der tat schwierig, eine DeFinition 24 Finden, die alle in Theorie und Praxis vorkommenden Modelitypen abdeckt. Zwei Beispiele mögen dies verdeutlichen:

- das Model1, das ein Architekt von einem geplanten Gebäude konstruiert;

- das sog. Gauß'sche RauchEahnenmodell, das im Rahmen der Ausbreitungsrechnung zur Prognase van Immissianen aufgrund bekannter Emissionen verwendet wird.

Diese Modelle unterscheiden sich in vielerlei Hinsicht, unter anderem im gewahlten Medium und in der Problemstellung, in deren Rahmen sie entwickelt wurden.

Das erste Modell ist aus einem materiellen Medium gefertigt (etwa Pappe und Klebstaff). Es dient dazu, dem Betrachter einen visuellen Eindruck des geplanten Bauwerks zu vermitteln.

Beim Gauß'schen RauchEahnenmodell handelt es sich um die sumbolische Repräsentation bestimmter physikalischer Zusammenhänge, die im Rahmen bestimmter mathematischer Konventionen als Rechenvorschrift (Algorithmus) aufgefasst und ausgewertet werden kann. Es dient zur Erstellung van Immissionspragnasen.

Was ist den beiden Modellen nun gemeinsam? Marvin MINSKY hat das charakteristische Merkmal aines Modells wie Eolgt umschrieben:
"An object $A$ is a model of an object $B$ if an observar $C$ can use A to answer questions that interest him about B." [MINSKY 6B]

Nach dieser pragmatischen Betrachtungsweise kann somit ein nicht unabhàngig von einem Beabachter (oder Anwender) und konkreter Fragestellung als ein Modell bezeichnet werden 
künte etwa lauten: Welche zusätzliche Schadstoffbelastung entsteht Für Mensch und Umwelt bei Inbetriebnahme aines Kohlekraftwerkes?).

Bei einem Eormalen Model1 wird die Beantwortung der Fragestellung durch Ausführung eines Algorithmus erreicht. Den (abstrakten) Algorithmus bezeichnet man in der Regel als konzeptuelles Modell (conceptual model), das Programm, das zu seiner Implemention auf einer konkreten Rechenanlage erstellt wird, als Computermodell (computerized madel) [SCS 79].

\section{Simulation:}

Auch der Begriff der Simulation wird recht unterschiedich definiert, PRITSKER hat in einem Beitrag in der Zeitschrift "Simulation" 22 Definitionen zusammengestelit [PRITSKER 79].

Diesem Beitrag soll folgende - relativ allgemeine - Definition zugrundegelegt werden:

Simulation ist der vargang der Generierung von
Modeliverhalten.

Bei einem Simulationsexperiment durchläuft ein Modell - abhängig van bestimmten experimentellen Bedingungen - eine Falge von Zustanden, die entsprechenden Zustanden des Realsystems zugeordnet werden kännen. Als Modellverhalten bezeichnet man die Menge aller möglichen Zustandsfolgen. Das gesamte Modellverhalten kann in des Regel aus Aufwandsgründen nicht generiert werden; man muß sich deshalb darauf beschränken, unter ausgewählten experimentellen Bedingungen zu simulieren.

Es ist typisch für die Simulation, daß die gewünschte Zielaussage erst durch eine - oft aufwendige - Auswertung der Simulationsdaten gewannen werden kann. Sie dient zur Reduktion der gewonnenen Daten auf die im Rahmen der Fragestellung interessierenden Aspekte.

Das Gegenstück zur Simulation bildet die analutische Berechnung. Hier wird das Ergebnis in einem Schritt cd.h. ohne iterative Fortschreibung von Modelizuständen) ermittelt, und es werden nur die Für die Zielaussage relevanten Daten erzeugt.

Die Simulationsmethode wird dann eingesetzt, wenn ein Realsystem aufgrund seiner Komplexität durch ein analytisches Verfahren nicht mehr adäquat abgebildet werden kann.

Die Simulation hat unabhängig davon den Vorteil, daß das Modellverhalten anschaulicher dargestellt wird. Simulatiansmodelle sind daher gut geaignet für Problemstellungen, bei denen das Verhalten eines Realsystems einem Beobachter demanstriert werden soll, also Z.B. im pädagogischen Bereich. 


\section{Klasegifikation von Moded den}

Modelle lassen sich nach nach ihrer Anwendung oder nach der ihnen zugrundeliggenden Methode klassifizieren.

AuE dem Umweltsektor kann man nach ihree Anwendung etwa die Ealgenden Modelitypen unterscheiden cohne Anspruch auf Uollständigkeit oder Uberschneidungsfreiheit):

- Ausbreitungsmodelle (sie dienen zur Berechnung von Immissionen auf der Basis bekannter Emissionen)

- Wasserbeschaffenheitsmodelle csie berechnen die wichtigsten Indikatoren Für den Zustand eines Gewässers in Abhängigkeit van Einleitungen und anderen Einflüssen)

- Belastungsmodelle (sie berechnen Belastungsindizes, d.h. kanstruierta Gräßen zur Beschreibung der Umweltbelastung, auf der Basis gemessener Einzelbelastungsfaktaren)

- bkologische Modelle (sie beschreiben die Beziehungen innerhalb eines bkosystems, d.h. die wechselwirkungen der in einem bestimmten Lebensraum lebenden Populationen untereinander und mit ihrem Lebensraum)

- b̈konomische Modelle (sie modellieren äkonomischer Aspekte des Umwel tschutzes)

- integrierte Umweltmodelle (sie beschreiben Wechselwirkungen zwischen verschiedenen Bereichen der Umwelt, wirtschaft, Politik usw. )

(Zur Modelibildung auf dem Umweltsektor s. auch [HILTY B6a].)

Nach der zugrundeliegenden Methode künnen Modelle nach einer Uielzahl von Kriterien klassifiziert werden. Im folgenden werden vier Kriterien beschrieben, die in der Literatur häufig genannt werden; sie werden jeweils am Beispiel von Umweltmodellen kankretisiert.

Ein erstes Kriterium ist die Unterscheidung zwischen

- analytischen Madellen,

- kantinuierlichen Simulationsmodelien und

- diskreten Simulationsmodellen.

Analytische Modelle unterscheiden sich von Simulatiansmadellen in der Form, in der die im Realsystem bestehenden (oder vermuteten) Beziehungen repräsentiert werden.

Bei einem analutischen Mode1l werden die Beziehungen zuischen den Komponenten des Ralsystems in Form aines analytisch lüsbaren G1eichungssystems repräsentiert. 
Bei einem Simulationsmodell können die Beziehungen dagegen prinzipiell in beliebiger Form repräsentiert werden. Es ist dann die Aufgabe einer Ablaufsteuerung, auf der Basis dieser Repräsentation das Modellverhalten zu generieren. Die Ablaufsteuerung kann je rach Madelityp unterschiedlich komplax sein. Insbesondere stellt die Implementation von kontinuierlichen bzw. diskreten Simulationsmodellen unterschiedliche Anforderungen an die Ablaufsteuerung.

Der zustand eines kontinuierlichen Simulationsmodelis andert sich kontinuierlich, der eines diskreten simulationsmodelis dagegen sprunghaft zu diskreten Zeitpunkten.

Bei der Implementation eines kontinuierlichen Simulationsmodelis auf einer digitalen Rechenanlage ist eine Diskretisierung der Zeitachse erforderlich.

Analytische Modelie und kontinuierliche Simulationsmodelle werden auf dem Umweltsektor häufig eingesetzt. Das erwähnte Gauß'sche RauchEahnenmodell etwa ist analytisch. Wie für solche Modelle typisch, ist es daher nur unter recht restriktiven Randbedingungen anwendbar. So muß u.a. vorausgesetzt werden, daß sich die windrichtung im Berechnungszeitraum nicht ändert, daß der Emissionsmassenstrom Eür einige Zeit konstant bleibt, daß das Gelände eben und wenig strukturiert ist usw. (Siehe hierzu auch [DITTMANN 86].) Sind diese Voraussetzungen nicht gegeben, müssen kontinuierliche Simulationsmodelle (numerische Ausbreitungsmodelle) herangezagen werden. (Einen Uberblick gibt [BLDCK BG].)

Entsprechendes gilt Eür Ausbreitungsmodelle, die im Wasserberaich angewandt werden, sowie für wasserbeschaffenheitsmodelle. Auch hier sind analytische Modelle an vereinfachende Annahmen gebunden (z.B. Vernachlässigung der Populationsdynamik im Gewàsser). Dil Modelilierung biozónotischer Prozesse geschieht dagegen mit kontinuierlichen Simulationsmadellen.

Ausschließlich diskrete Simulationsmodelle spielen auf dem Umweltsektor eine geringe Rolle. Die Kombination von kontinuierlichen und diskreten Modellen kann dagegen häufig sinnvoll sein. CELLIER [B4] beschreibt als Beispiel die Simulation eines solarenergie-Heizsystems. Hier sind sowohl diskrete (Sonnenauf - und untergang, Hinzuschalten einer blheizung usw.) als auch kontinuierliche Zustandsänderungen (Ansteigen der Temperatur usw. z zu modellieren.

Ein zweites Kriterium ist die Unterscheidung zwischen statischen und dynamischen Model len.

Ein dunamisches Modell beschreibt die Veränderung von Eigenschaften des Realsystems im Zeitverlauf, während ain statisches Modell von Veränderungen abstrahiert. 
Ein statisches Modell besitzt somit keine zeitabhängige Dutputvariable, ein dynamisches Modell dagegen mindestens eine.

Als Beispiel sei hier wiederum das Gauß-Modell herangezogen. Es ist statisch, da die Annahme gemacht wird, daß die Konzentration an einem bestimmten Punkt des Raumes unter bestimmten Randbedingungen kanstant bleibt. Daran ist 24 erkennen, daß statische Modelle durchaus dynamische Realphänomene (in diesem Fall die Ausbreitung von Schadstoffen in der Atmosphäre) beschreiben können. (Strenggenommen ist - bei genügend detaillierter Betrachtung oder bei genügend weitem Zeitrahmen jedes Realsystem dynamisch.) Ein statisches Modell kann als "Momentaufnahme" eines Realsustems aufgefaßt werden, wobei eine zeitliche Aggragation über dem Beobachtungszeitraum statteindet [Vgl. HANSSMANN 78].

Dynamische Modelle sind auf dem Umweltsektor in Fast allen Bereichen - van numerischen Ausbreitungsmadellen bis zu integrierten Umweltmodellen - vorzuFinden.

Simulationsmodelle sind grundsatzlich dynamisch. Analytische Modelle sind meist statisch; in geuissen Grenzen lassen sie jedoch auch die dynamische Modellierung $z u$.

Ein drittes Kriterium ist die die Anzahl der Raumimensionen, die ein Modell berücksichtigt.

Ein Modell heißt räumlich $n$-dimensional, wenn $n$ die maximale Anzahl der Raumdimensionen ist, von denen eine Dutputvariable abhängig ist.

Ausbreitungsmodelle, die im Rahmen der Luftreinhaltung eingesetzt werden, sind 3-dimensional. Im Wasserbereich genügen meist 1 - oder 2-dimensionale Ausbreitungsmodelle. Die räumliche Uerteilung bestimmter Größen spielt auch bei Belastungsmodellen eine zentrale Rolle. Ein solches Modell berechnet (ausgehend von gemessenen Belastungsfaktaren) Belastungsindizes für ein bestimmtes geographisches Gebiet. Die Ausgabe erfolgt in Form van $2-$ dimensionalen Belastungskarten (Raster- oder Isoliniendarstellung der Umwel tbelastung).

Schließlich ist zwischen deterministischen und stochastischen Modellen zu unterscheiden. Ein deterministisches Modell besitzt keinen
stochastischen Zustandsübergang; oin stochastisches
Madell mindestens einen.

Ein stochastischer Zustandsübergang liegt dann vor, wenn der auf einen Zustand $z_{n}$ Folgende Zustand $z_{n+x}$ durch $z_{n}$ nicht eindeutig Eestgelegt ist. 
Db ein Realsystem deterministisch modelliert werden kann, ist letztlich eine Frage des Aggregationsniveaus. Bei ausreichend detailierter Betrachtung (niedriges Aggregationsniveau) verhält sich Jedes Realsystem stochastisch.

Stochastische Modelle kommen im Umueltbereich $u . a$. in der Ausbreitungsrechnung zur Anwendung. Es werden hier einzelne Massepartikel auf ihren Trajektorien verfolgt, wobei die Bewegungsrichtung der Teilchen nur statistisch angegeben werden kann [VG]. BLOCK BG].

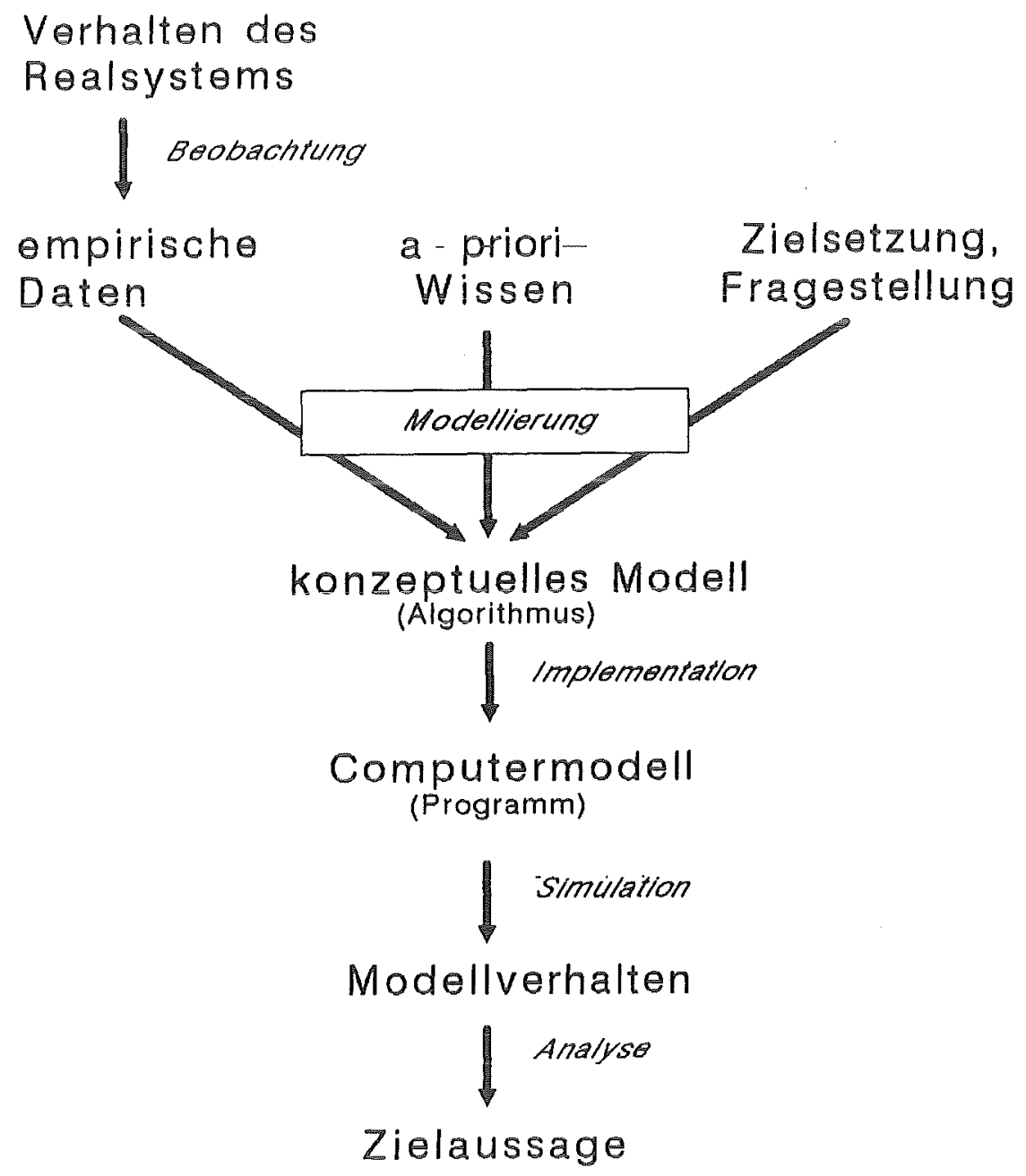

Abb. 1: Simulationsstudie 


\section{Konzepte zur Unterstützung der Modellbildung und Simulation}

Wird der Camputer als Modellmedium benutzt, so kann die Modelibildung und Simulation auf vielfälige Weise unterstützt werden. Abb. 1 zeigt (stark schematisiert) die verschiedenen Phasen einer Simulationsstudie.

In der Phase der Modellierung kann der Rechner zur Verwaltung und Analyse der über das Realsystem vorliegenden Daten sowie als Medium für informale Beschreibungen des konzeptuelien Modelis (Texte, Graphiken) dienen.

Die Implementation des Modelis wird am besten durch Simulationssprachen unterstützt, welche es erlauben, das Modell in problemadaquaten Sprachkonstrukten zu formulieren.

Die Simulation kann durch Bereitstellung einer leistungsfähigen und Flexiblen Ablaufsteuerung unterstützt werden. Der Benutzer solite Ferner die Möglichkeit haben, komplexere Simulationsexperimente (z.B. Experimente mit systematischer Parametervariation) auf ohne großen Aufwand zu spezifizieren.

Zur analuse des Modellverhaltens schließlich können vordefinierte oder vom Benutzer implementierte Methoden zur Auswertung und Darstellung der Simulationsdaten vorgesehen werden.

Alle genannten Möglichkeiten der Unterstützung sollten interaktiv nutzbar sein und dem Benutzer in einem einheitlichen, kansistenten Rahmen angeboten werden.

Zwei Softwarekanzepte, die dies prinzipiell leisten können, sind das interaktive Simulationssustem und das Modellbanksustem.

In den Folgenden Abschnitten wird zunächst auF Simulationssprachen eingegangen; anschließend werden die genannten interaktiven Konzepte beschrieben.

\section{1 Simulationseprachen}

Simulationssprachen lassen sich nach zunehmender Problemorientierung wie folgt klassifizieren [SCHMIDT 79]:

- niedere Simulatianssprachen

- höhere Simulationssprachen

- systemorientierte Simulatianssprachen

Eine niedere Simulationssprache erleichtert die Implementation von Simulationsmodellen durch Sprachelemente, die eine kompakte Farmulierung simulationstunischer Pronrammteile erlauben (Ablaufsteuerung, ZuFallszahlengenerierung, Protokolilerung von Ergebnissen usw.).

Beispiele: SIMULA, GASP, SIMSCRIPT 
Höhere Simulationssprachen sehen zusätzlich Sprachelemente zur kampakten Formulierung van Modelikamponenten vor, dia im Rahmen bestimmter Problemstellungen häufig benítigt werden $(z . B$. Warteschlangen bei diskreten, Flußraten und Zustandsgräßen bei kontinuierlichen Simulationsmodellen).

Beispiele: DYNAMO, GPSS, SIMON, PASSIM

Systemorientierte Simulationssprachen sind noch starker problemorientiert. Sie enthalten Sprachelemente, welche die Modellierung bestimmter Sustemklassen unterstützen (z.B. Rechensysteme, Eisenbahnnetze, Krankenhäuser).

Beispiele: IPSS/BDMS, RAILSIM, HOSPSIM

Erfahrungen haben gezeigt, daß durch die Verwendung von Simulationssprachen anstelle von allgemeinen höheren Programmiersprachen (wie FURTRAN, PASCAL USW.) sich der Implementationsaufwand Für Modelle erheblich verringern läßt [SCHMIDT 79].

Dies gilt jedoch stets nur Eür bestimmte Madelle. So besteht bai Verwendung einer stark problemorientierten Sprache die Gefahr, daß Modelie mit einer nicht "sprachkonformen" Struktur den Möglichkeiten der Sprache angepaßt - oder gar nicht erst konzeptuell entwickelt - werden.

\subsection{Interaktive simulationssysteme}

Solche Systeme unterstützen den Benutzer interaktiv in verschiedenen Phasen einer Simulationsstudie.

In der Regel formuliert der Benutzer sein Modell mit Hilfe einer Simulationssprache und bettet sein Modell durch Spezifikation einer Schnittstelle in das Rahmensystem ein (Abb 2 ). Wichtig ist, daß Modifikationen am Modeli leicht vorzunehmen sind und sich der anschließende Wechsel zur Phase des Testens und Experimentierens rasch und unkompliziert vallzieht.

Das Experimentieren selbst (einfache Simulationsläufe oder komplexere Experimente, welche mehrere Läufe einschließenJ geschieht im Dialog mit dem System, wobei die experimentellen Bedingungen (etwa die Eingabedaten für das Modell) interaktiv spezifiziert werden können.

Zur Analyse des Ergebnisses steht ein Auswertungssystem zur Uerfügung, welches dem Benutzer vordefinierte Standardmethoden zur Datenauswertung und -darsteliung anbietet. Diese sind entueder interaktiv nutzbar (Ergebnisanalyse im "Tischrechnermodus"), oder sie werden var Beginn des Experiments ausgewählt und erlauben somit die begleitende Auswertung und Ausgabe der Ergabnisse.

Beispiele Eür interaktive Simulationssysteme sind: ASS [BOSSEL 81], BAHSIM [BAH 83], SISSY [RETTI 82], DYNAMIS [HÄUSLEIN 86]. 


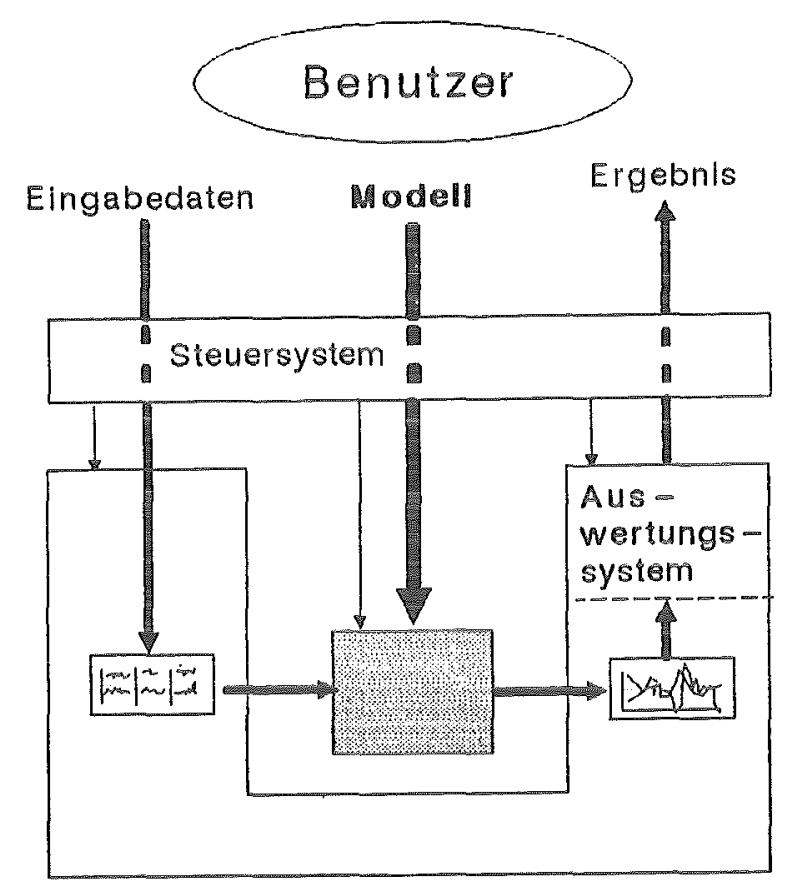

Abb. 2: Interakilves Simulationssystem

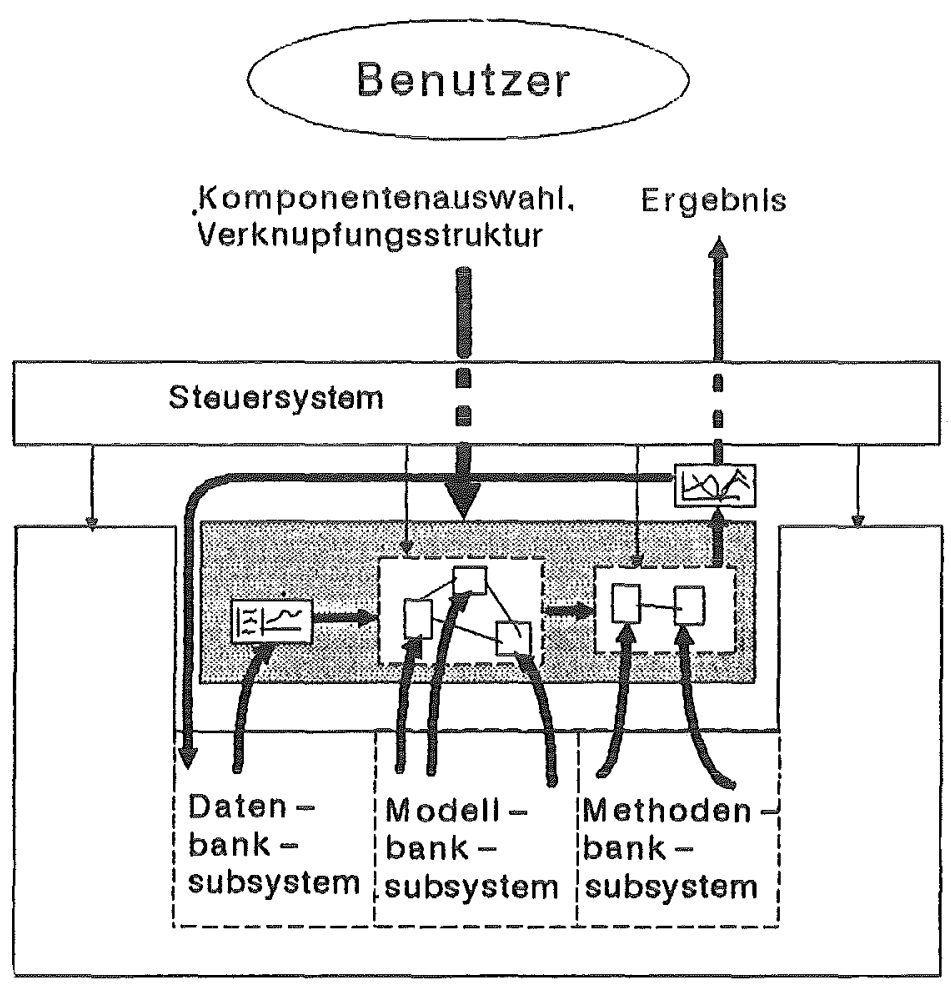

Abb. 3: Modellbanksystem 


\section{3 Model 1banksysteme}

Ein Modellbanksystem ermbglicht dem Benutzer den Aufbau einer Sammlung von Modelibausteinen, die er im Rahmen wechselnder Problemstel lungen jeweils neu verknüpfan und parametrisieren kann ("Baukastenprinzip"). Eine salche Modellbank kann auch einem gräßeren Benutzerkreis zugànglich gemacht werden, wobei wie bei einem Datenbanksystem verschiedene Benutzersichten definiert werden können.

Neben Modelien verwaltet ein Modelibanksystem auch Methoden ( $2 u r$ Auswertung und Darstellung von Datenl und die innerhalb des jeweiligen Anwendungsbereiches benötigten Daten. Man kann ein Modellbanksystem entsprechend in 3 Subsysteme einteilen (Modell-, Methoden- bzw. Datenbank-Subsystem; 5 . Abb 3).

Im Idealfall sind alle Komponenten, die ein Benutzer für seine aktuelle Problemsteliung benötigt, zum jeweiligen zeitpunkt bereits im System vorhanden. Im Dialog mit dem System waihlt er diese Komponenten aus und spezifiziert deren Verknüpfungsstruktur. Das entstehende Gebilde aus Eingabedaten, Modellkomponenten sowie Ausuertungs- und Darstellungmethoden erzeugt ein Ergebnis, das wiederum im Datenbank-Subsystem abgelegt wird.

Der Bestand an Model1- und Methodenbaustejnen sowie Daten kann vom Benutzer erweitert und somit laufend dem zunehmenden wissen über das Anwendungsgebiet und neuen Anforderungen angepaßt werden.

Die Iatsache, daß sich das Konzept des Modellbanksystems in der Praxis nicht durchgesetzt hat, mag verschiedene Ursachen haben. Eine Ursache ist wohl darin zu sehen, daß es wenige Anwendungsgebiete gibt, in denen sich eine konsistente Sammlung von weitgehend validierten Modellen aufbauen läßt.

Dieses Problem läßt sich allerdings lösen, wenn man bei Modelibanksystemen (anders als bei Datenbanksystemen) keine Kansistenz Fordert und damit auch widersprüchliche Ergebnisse in Kauf nimmt. Die begrenzte Validität eines Modelis wäre im System explizit zu repräsentieren. ZEIGLER spricht in diesem Zusammenhang von "competitive models" [ZEIGLER B4].

Gerade auf dem heterogenen Feld der "Umueltmodellierung" erscheint ein solches Konzept angemessen. Es wäre sicher von großem Nutzen für einen Umweltplaner, wenn er beispielsweise verschiedene Ausbreitungsmodelle nebeneinander in einem Modellbanksystem zur Verfügung hätte: Führen die Modelle im Rahmen einer bestimmten Fragestellung zum gleichen Ergebnis, wird das Vertraven des Benutzers in das Resultat um so größer sein. Ergibt sich dagegen ein widerspruch, ist der Anwender gewarnt und wird sich verstärkt um die Frage der Validität der Modelle in Bezug auf seine spezielle Fragestellung kümmern.

Das einzige im praktischen Einsatz befindliche Modelibanksystem ist das van der GMD entwickelte MBS [GMD 83]. Der Autor hat die 
Eignung von MBS für die Anwendung auf dem Umweltsektor untersucht und ist zum Ergebnis gekommen, daß wohl das Konzept des Model lbanksustems. nicht aber das konkrete Sustem MBS für dieses Anwendungsgebiet geeignet wären [HILTY 85]. Die Entwicklung eines auf dem Madelibankkonzept beruhenden "Umweltmodeliierungssystems" steht bisher noch aus.

\subsection{Anforderungen an Model 1bildungs-/Simulationswerkzeuge}

Obwohl die beschriebenen Konzepte nicht neu sind und (mit Ausnahme des Modellbankkonzeptes) vielfach realisiert wurden, ist es - gerade auf dem Umweltsektor - verbreitete Praxis, Simulationsmodelle mit relativ unspezifischen Hilfsmitteln zu implementieren.

Dies liegt nach Ansicht des Autors daran, daß die gängigen Modelibildungs- und Simulationswerkzeuge einige wesentliche Anforderungen nicht erfüllen, die an ein komfortables Werkzeug zu stellen sind. Anstatt sich mit komplizierten Sprachen und Dialogsystemen anzufreunden, die letztendlich doch nicht den erwünschten Komfort bieten, zieht sich der Praktiker lieber auf die altbewahrten Hausmittel zurück.

Die Ealgenden Anforderungen sollten von einem adaquaten Modelibildungs- und Simulationswerkzeug erfülit werden:

1. Ausreichende Strukturierungsmöglichkeiten

Es sollte dem Benutzer möglich sein, das Modell in Submodelle zu gliedern, wobei potentiell beliebig viele Hierarchieebenen zur Werfügung stehen.

Ein Negativbeispiel hierzu ist die Simulationssprache DYNAMO, die keine Strukturierung von Modellen ermöglicht. Ein weiteres Negativbeispiel ist das Modellbanksystem MBS, das genau 2 Hierarchieebenen (Für "Modelle" und "Partialmodelle") vorsieht. Es ist unwahrscheinlich, daß gerade diese Modelistruktur für alle Problemstellungen optimal ist.

2. Adäquate Datenstrukturen

Das System sollte für die Modellbildung und Simulation spezifische Datenstrukturen vorsehen. Dazu gehören Parametervektoren und Zeitreihen. Eine Zeitreihe ist eine sequentiell zugreifbare Datenstruktur mit einheitlichem, aber beliebigem Grundtyp und den Attributen "AnEangszeitpunkt", "Endzeitpunkt" und "Periodizitä". (Dadurch unterscheidet sie sich etwa von der struktur "File").

3. Robustheit

Unter der Robustheit wird hier die Fähigkeit des Systems verstanden, semantisch unsinnige Dperatianen (dia durch 
Fehler im konzeptuelien Model1, durch Implementationsfehler oder durch Rechenungenauigkeiten zustande kammen können) möglichst weitgehend durch geeignete Uarkehrungen auszuschließen. Maßnahmen zur Verbesserung der Robustheit sind Z.B. (vgl. [CELLIER B4]):

- Wertebereiche für Modelivariablen (dadurch künnte z. $\bar{B}$. eine Modellvariable "Bevölkerung" dagegen gesichert werden, negative Werte anzunehmen - viele Simulationssprache lassen das nicht zu);

- Maßeinheiten Eür Modellvariablen und alle Ein- und Ausgabedaten czu jeder im System repräsentierten Gräße hat der Benutzer eine Maßeinheit anzugeben; das System kann dann die Korrektheit der Rechenoperationen hinsichtlich der Maßeinheiten prüFen).

4. Trennung von Mode11 und Experiment

Bei der Implementation eines Simulationsmodells müssen sowohl die Struktur des konzeptuellen Modelis als auch die damit durchzuführenden Experimente spezifiziert werden. Da dies häufig innerhalb desselben Programms geschieht, ist vielen Modelientwicklern der prinzipielle Unterschied zwischen Modelien und Experimenten nicht bewußt. Ein gutes Werkzeug solite den Benutzer veranlassen, modellbezogene Programmteile (etwa Modellgleichungen) van experimentbezagenen Teilen (Ablaufsteuerung, Datenzuweisung, Ausgabe usw.) mäglichst unabhängig $z u$ implementieren (soweit die letzteren nicht weitgrhend vordefiniert sind oder interaktiv spezifiziert werden können).

5. Unterstützung Komplexer Model lexperimente

Das Experimentieren mit Simulationsmodellen beschränkt sich nicht auf einfache Simulationsläufe. Oft ist es wünschenswert, Modeliparameter oder Inputvariablen systematisch zu variieren und Simulationsläufe wiederholt auszuführen ( $Z$.B. im Rahmen einer Sensitivitatsanalyse). Auch solche Experimente sollten komfortabel durchgeführt werden kännen.

6. Unterstützung der Dokumentation von Model len und Daten

Die sorgfältige Dokumentation eines Modelis und allen von ihm benutzten und erzeugten Daten ist die Uoraussetzung Eür seine Anwendbarkeit und Transparenz für Aussenstehende. Zur Dokumentation können natürlichsprachliche Texte und Grafiken dienen. Salche dokumentierenden abjekte sollten vom system verwaltet werden; auch ihre Beziehung $z u$ den dokumentierten Qbjekten sollte im System repräsentiert (und nicht dem Benutzer überlassen) sein. 
7. Uerknüpfung von Model len unterschiedlichen Iups

Viele Realsysteme lassen sich nur durch die Aufteilung in Subsysteme adäquat modeliieren, welche mit unterschiedlichen Modelitypen abgebildet werden. Insbesondere die Kombination von kontiniverlichen und diskreten Simulationsmodellen sowie von analytischen Modellen mit Simulationsmodelien sollte vam system unterstützt werden.

8. Autamatische IransEormation zwischen unterschiedlichen Darstellunasformen

Sowohl Modelle als auch Daten lassen sich in unterschiedlicher Form darstellen ( $z . B$. als Programm bzw. Tabelle und als Graphik). Ein kamfortables Werkzeug sollte möglichst weitgehend zwischen diesen Darstellungsformen transformieren können, wobei es dem Benutzer Ereigestellt ist, in welcher form er das betreffende objekt editieren möchte. So ist es $z . B$. denkbar, ein System-Dynamics-Diagramm automatisch in das entsprechende DYNAMO-Programm zu transformieren, wobei fehlende Angaben interaktiv nachgefordert werden. (Das gleiche gilt für andere Modellierungsmethoden, die Modelldiagramme verwenden.)

9. Benutzergerechte Bedienungsoberfläche

Diese Forderung ist selbstverstandlich an alle interaktiven Systeme zu stellen; sie ist also nicht spezifisch für Modelibildungs- und Simulationswerkzeuge. Dennoch soll dieser Aspekt hier unterstrichen werden, denn er ist von entscheidender Bedeutung, wenn es mäglich sein soll,

- daß z.B. Bin dkologe ahne spezielle EDU-Kenntnisse ein Simulationsmodell entwickelt,

- daß z.B. ein Umweltplaner ohne spezielle EDU-Kenntnisse ein vorhandenes Simulationsmodell anwendet, um die Auswirkungen verschiedener hypothetischer Maßnahmen besser einschätzen zu können, oder

- daß z.B. ein Lehrer ohne spezielle EDU-Kenntnisse im Biologieunterricht die Eutrophierung eines Sees anhand eines Simulationsmodel is demonstriert.

Leider kann hier nicht in der nötigen Ausführlichkeit auf die Gestaltung von Benutzerschnittstellen eingegangen werden. Als Beispiel sei nur das Prinzip der direkten Manipulation erwähnt, das sich heute durch den Einsatz entprechender Hard- und Systemsoftware relativ kostengünstig realisieren läßt.

Dieses Prinzip wurde beim Simulationssystem DYNAMIS [BRUNNSTEIN B6; HäUSLEIN 86] verwirklicht ( 5 . Abb 4). Auf dem Bildschirm sind drei Fenster zu sehen, deren Umfang und Lage beliebig verändert werden kann. Das erste Fenster zeigt ein Modell in Form eines System-Dynamics-Diagramms, das dahinterliegende 


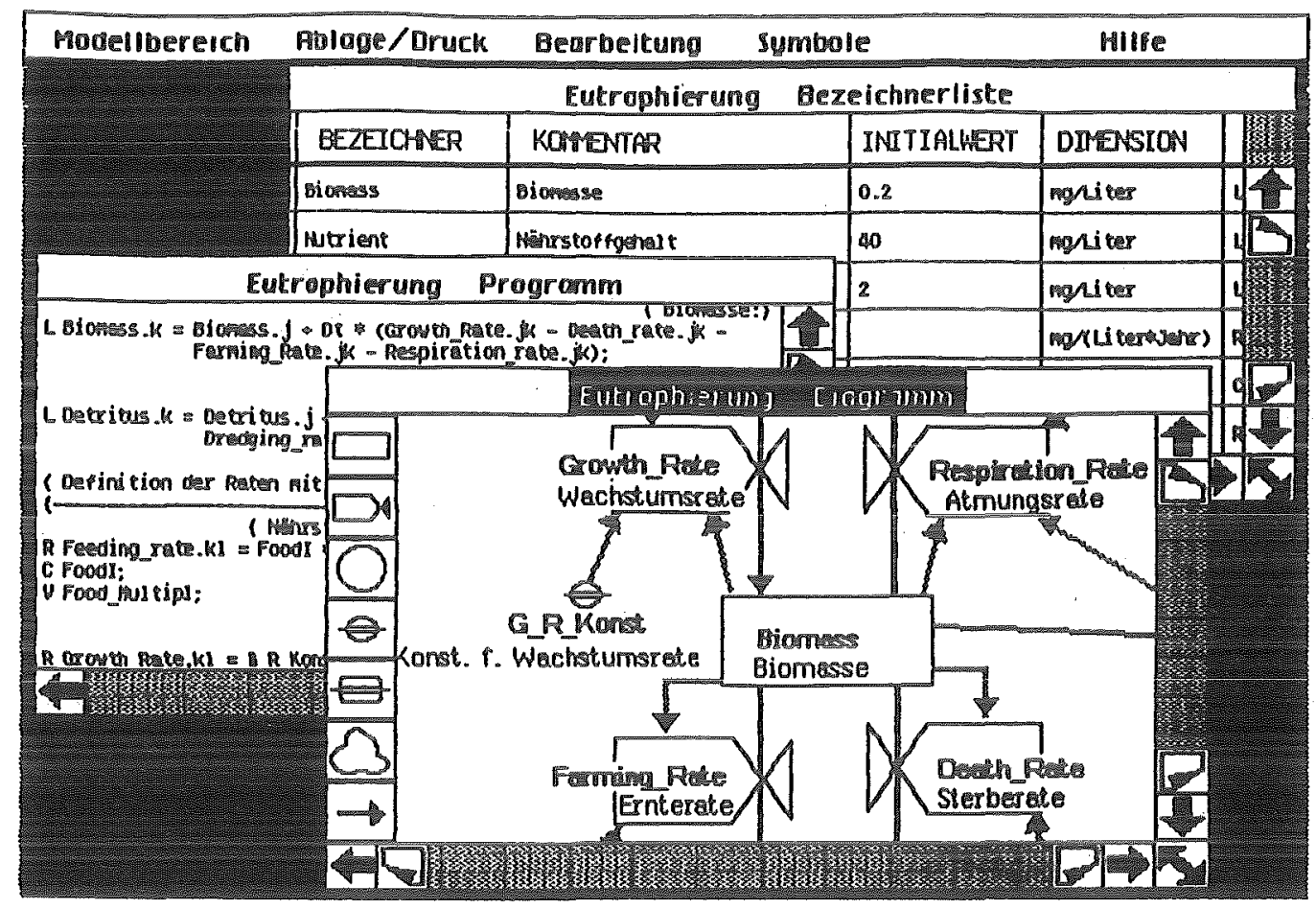

Abb. 4: Benutzeroberflåche des Simulationssystems DYNAMIS

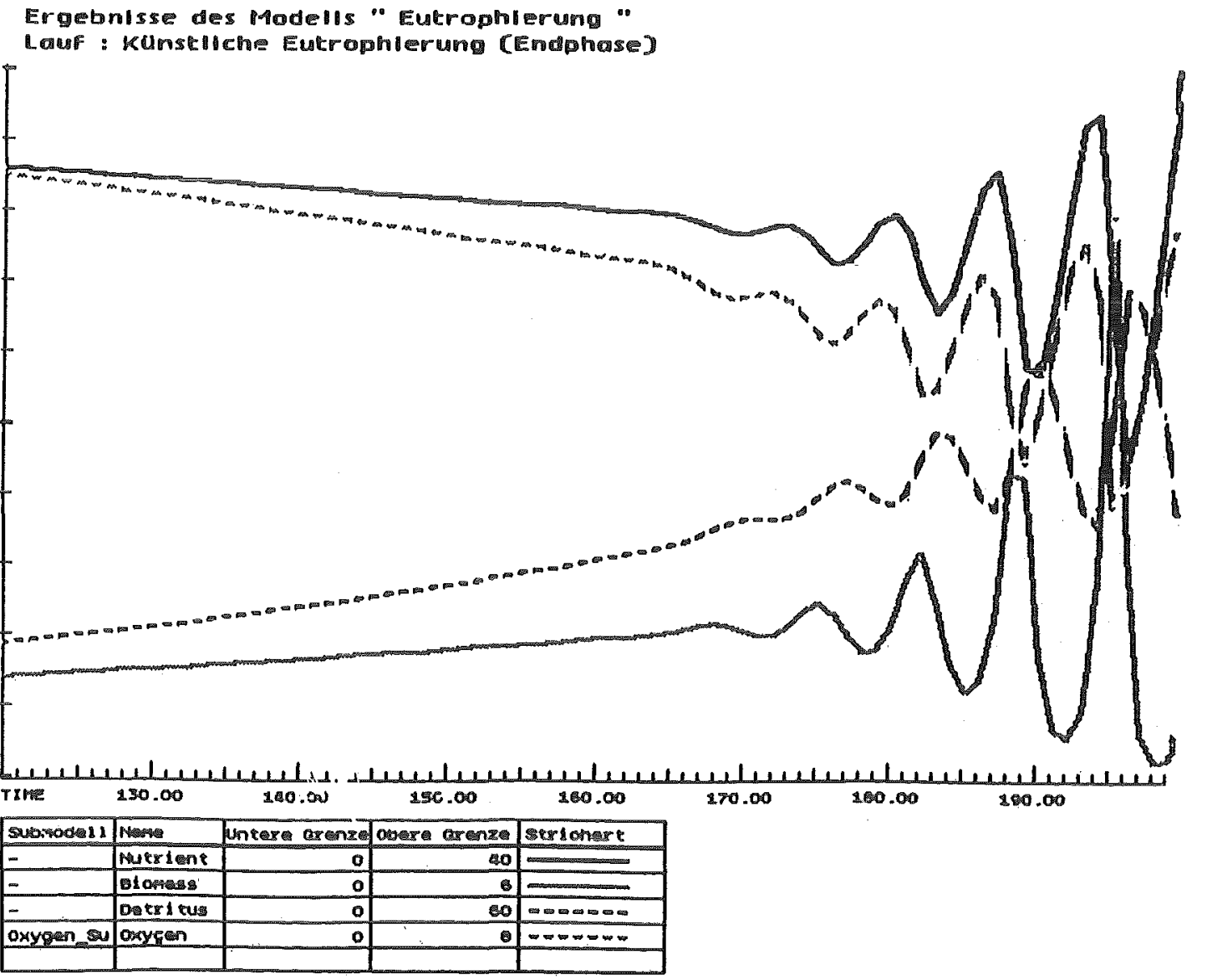

Abb. 5: Simulationslaui 
Fenster das entsprechende DYNAMO-Programm (es handelt sich um ein erweitertes DYNAMO, das die Strukturierung von Modellen ermäglicht). Im dritten Fenster schließlich ist die sog. Bezeichnerliste zu sehen, die dem Benutzer einen Uberblick über alle Modellgraßßen, ihre Bedeutung, ihren Initialwert beim aktuellen Simulationsexperiment und ihre Dimension gibt. Die gezeigten Dbjekta können am Bildschirm - mit Hilfa der Maus direkt editiert werden.

Abb. 5 zeigt die Darstellung von Simulationsergebnissen mit DYNAMIS. Die Ausgabe erfolgt begleitend, d.h. bereits während des Simulationslaufs.

\section{Simulation und Künstliche Intelligenz}

In jüngerer Zeit sind eine Reihe von Publikationen erschienen, die sich mit einer möglichen Uerbindung von konventionellen Simulatiansmethoden mit Techniken aus dem Bereich der Künstlichen Intelligenz (KI) befassen. Dffanbar besteht die Hoffnung, daß einige Klassische Probleme der Simulation mit Hilfe der KI gelöst werden können, und daß ungekehrt bestimmte Anwendungsbereiche der KI (etwa der Bereich Expertensysteme) van Simulationstechniken profitieren können.

An dieser Stelle soll nur der erste Weg - der Einsatz von KIMethoden in der Simulation - stichwortartig skizziert werden. Es sind vor allem drei Bereiche, in denen die Simulation von der KI profitieren könnte:

\section{Wissensrepräsentation}

Ein Experte, der ein Simulationsmodell entwickelt, farmalisiert dabei sein Wissen über ein Realsystem Coder eine Klasse von Realsystemen) und repräsentiert os im (Simulations-)System. Das gleiche Ziel wird im Rahmen der KI mit der Konstruktion von Expertensystemen verfalgt. In beiden Fällen wird dabei das System als ain aktives Medium benutzt. Ein Unterschied besteht jedoch darin, $d a \beta$ ein Expertensustem aus dem eingegebenen Wissen Schlüsse zieht, daß also neues Wissen aus vorhandenem Wissen erzeugt wird, während ein Simulationssustem den (sehr viel spezielleren) Schritt von der Modelistruktur zum Model lverhalten vollzieht.

Es könnte für die Simulation nützlich sein, Modelle in einer Form zu repräsentieren, die nicht nur für die Ablaufstouerung interpretierbar, sondern auch Inferenzprozessen zugänglich ist. Das System wird dadurch in die Lage versetzt, Aussagen über Modelie zu machen ( $z . B$. Inkonsistenzen aufzudecken) oder Madelle selbst zu modifizieren. 


\section{Suchuerfahren}

Im Gegensatz zur analytischen Modellierung stellen sich bai der Simulation Suchprobleme $(z, B$. das Problem der Model1kalibrierung: "Bei welcher Parameterkombination entsprechen meine Simulationsdaten den empirischen Daten am besten?"). Hierzu sind heuristische Suchverfahren erforderlich, dil u.a. auch im Rahmen der KI erfarscht werden.

3. Benutzerdialog

Der Umgang eines Benutzers mit Simulationsmodelien, die er nicht selbst entwickelt hat - die ihm z.B. im Rahmen eines Modellbanksystems angeboten werden - ist wünschenswert, wirft jedoch eine Reihe von Problemen auf.

So muß der Benutzer zunächst dabei unterstützt werden, ein für seine spezielle Fragestellung relevantes Modell aufzufinden. Es muß ihm ferner die relative Validitat eines Modells vermittelt werden. Die Annahmen, auf die es sich stützt, die Randbedingungen, unter denen es anwendbar ist, sowie die Ergebnisse bisheriger Validierungsbemühungen müssen transparent gemacht werden.

Schließlich muß der Benutzer beim Experimentieren mit dem Modell und der Ergebnisanaluse qualifiziert beraten werden. Welche Für das Modell relevanten Daten stehen zur Verfügung (z.B. als Eingabe- oder Vergleichsdatenj? Wie vollständig, wie zuverlässig sind diese? Welche Methoden eignen sich zur Auswertung und Darstellung der Ergebnisse?

Die KI künnte einiges zur Entwicklung eines in diesem Sinne "intelligenten" Systems beitragen.

\section{Chancen und Risiken der Simulation auf dem Umweltsektor}

Der Nutzen eines Simulationsmodells ist entscheidend vam UmEald abhängig, in dem es eingesetzt wird.

- Wird es im Rahmen der Grundlagenforschung zur Erklärung des Uerhaltens eines Realsystems eingesetzt?

- Wird es im Rahmen von Planungs- und Entscheidungsprozessen zur Abwägung verschiedener hypothetischer Maßnahmen verwendet?

- Wird es zu Instruktions- oder Demonstrationszwecken verwendet?

Im ersten Fall wird ein sehr hoher Anspruch mit der Entwicklung von Simulationsmodellen verbunden. Der Nachwais, daß ein Simulationsmodell das Verhalten eines Realsystems erklärt, dürfte auf seribse Weise schwierig zu erbringen sein. Doch selbst wo dies nicht gelingt, hat die Modelibildung den Nutzen, daß der 
Wissenschaftler seine Annahmen explizit formuliert und somit kommunizierbar macht. Dieser Effekt ist besonders bei interdisziplinären Modellstudien - und damit auf dem Umweltsektor - von Vorteil. Das simulationsmodell wird zu einem Kommunikationsmedium Für dia beteiligten Spezialisten; es dient dazu, das verstreut vorhandene Wissen explizit zu formulieren und in konsistenter form zusammenzuFügen.

Im zweiten Fall wird vom Modell gefordert, daß es das verhalten des Realsystems teilweise reproduziert cjedoch nicht notwendigerweise erklärt). Auch dieser Anspruch verlangt natürlich nach einer sorgfältigen Validierung.

Die zuletzt genannte Anwendung der Simulation birgt nach Ansicht des Autors die meisten ungenutzten Chancen: Gerade auf dem Umueltsektor läßt sich an Simulationsmodellen vieles anschaulich demonstrieren, das der Erwartung widerspricht: daß etwa varnetzte Systeme nicht immer in erwarteter weise auf äußeren Einwirkungen reagieren; daß das Zusammenwirken mehrerer Einflüsse einen anderen EFfekt hat als man aufgrund der Ausuirkungen der ainzelnen Einflüsse vermutet; daß auch sich selbst stabilisierende Systeme an die Grenzen ihrer Belastbarkeit gebracht werden können - die Liste ließe sich beliebig Eartsetzen. Allein salche allgemeinen Erfahrungen an simulierten Systemen können die realistische Einschätzung des aktuellen Umweltprobleme und die Beraitschaft zu einer differenzierten Betrachtungsweise erheblich unterstützen.

Mit der Anwendung von Simulationsmodellen sind aber auch Risiken verbunden. So besteht die Gefahr, daß ungeprüfte Annahmen und Schlußfolgerungen durch die Verwendung des Camputers als Modellmedium mit einer Scheinoblektivität ausgestattet werden. Unter Ausnutzung einer weit verbreiteten Computerglabuigkeit können einem breiten Publikum selbst reine Artefakte als Tatsachen dargestellt werden.

Schließlich besteht bei der Simulation von Umweltproblemen auch die Gefahr, daß die Modellbildung zum Selbstzweck wird, d.h. nicht mehr in Bezug auf die Konsequenzen bewertet wird, die sie für die wirkliche Umwelt hat oder haben kunnte.

Beispielsweise wird ein wissenschaftler, der an einer umfangreichen Simulationsstudie zum waldsterben arbeitet und täglich mit seinem Auto (ohne Katalysator) zur Arbeit fährt, damit wahrscheinlich größeren Schaden anrichten coder jedenfalls mitverursachen?, als er vermutlich jemals mit seinen Forschungsergebnissen wird abwenden können. Denn diese Ergebnisse haben nur dann einen Sinn, wenn daraus praktische Konsequenzen gezogen werden. Und dies erscheint extrem unuahrscheinlich angesichts der Tatsache, daß schon aus dem heute vorhandenen Wissen zum Problem weder von Palitikern noch Bürgern wirksame Konsequenzen gezogen werden. 


\section{Schlußfolgerung}

Es bestehen zahlreiche ungenutzte Chancen zur besseren Unterstützung der Modelibildung und Simulation durch geeignete Softwarekonzepte. Der Einsatz adaquater werkzeuge wïrde sich u.a. positiv auf die Transparenz von Modellen auswirken.

Ein "Umweltmodellierungssystem" hätte infolge der methodischen Vielfalt auf diesem Gebiet hohen Anforderungen zu genügen, soll ein verantwortungsbewußter Umgang mit Modellen gewährlaistet sein. Für die Konzeption eines salchen Systems wäre die Verbindung des Modelibankkanzeptes mit Techniken aus dem Bereich der Künstlichen Intelligenz eine sinnvalle Grundlage.

\section{Literatur:}

$[B A H \quad 83]$

Kartheus, $u_{.}$, Greve, $w$. , BAHSIM - Ein allgemeines SoftwarePaket mit Grafikoptionen zur leichten Programmierung von Simulationsmodellen in FORTRAN, Arbeitsvorschrift. Biologische Bundesanstalt Helgoland, Hamburg 1983

\section{[BLOCK B6]}

Block, H.-J., Numerische Ausbreitungsmodelle. In: [PAGE 06], S. $218-250$

[BOSSEL B1]

Bosse1, H., Dynamische Simulation mit dem

Modellerstellungsprogramm "ASS". In: Albertin, L., Müller, N., Umfassende Modellierung regionaler Systeme - Probleme, Modelle, Praxisbezug. ISR/TUU Rheinland 1981, S. 136-144

\section{[BRUNNSTEIN B6]}

Brunnstein, K., Mäuslein, A., Page, B., DYNAMIS: A System for the Interactive Support of Simulation Modelling and Experimentation. In: Proceedings of the 2nd European Simulation Congress, Antwerpen, 1986, S. 297-301

\section{[CELLIER B4]}

Cellier, F. E., How to Enhance the Robustness of Simulation Software. In: Uren, T. I., Zeigler, B. P., Elzas, M. L. (Hrsg.), Simulation and Model-Based Methodologies: An Integrative Uiew. Springer-Verlag, Berlin 1984, 5. 519-536

\section{[DITTMANN 86]}

Dittmann, J., Ausbreitungsmodelle in der Luftreinhaltung unter besonderer Berücksichtigung des Gauß-Modells. In: [PAGE B6] S. 197-217 
[GMD B3]

Klösgen, W., Schwarz, W., Hornermeier, A., Model1banksystem (MBS), Benutzer-Handbuch. Arbeitspapiere der GMD Nr. 32, Bann 1983

\section{[HANSSMANN 78]}

Hanssmann, F., Einführung in die SystemEorschung, Methodik der modeligestützten Entscheidungsfindung. aldenbourg, München 1978

[HÄUSLEIN B6]

Máuslein, A., Page, B., DYNaMIS: Ein Modellbildungs- und Simulationssystem mit objektorientierter Benutzeroberfläche. In: Hommel, G., Schindler, S. (Hrsg.), GI-16. Jahrestagung I, Proceedings, Springer, Berlin 1986, 5. 329-343

[HILTY 85]

Hilty, L., Benutzergerechte Made1lierungssysteme - Kriterien Für eine benutzergerechte Gestaltung von systemen zur Unterstützung der Modellbildung und Simulation. Diplamarbeit, Fachbereich Informatik, Universitat Hamburg 1985

\section{[HILTY B6a]}

Hilty, L., Page, B., Computeranwendungen im Umweltschutz Einsatzbereiche und Methoden - Ein Uberblick. In: [PAGE 86] S. $30-60$

\section{[HILTY BEb]}

Hilty, L., Page, B., Anwendungsmöglichkeiten von Methodenund Modelibanksystemen im Umueltbereich. In: [PAGE 日6] 5 . $331-347$

\section{[MINSKY 68]}

Minsky, M., Matter, Mind, and Models. In: Minsky, M. (Hrsg.), Semantic Information Processing, Cambridge Mass. 1968, 5. $425-432$

\section{[PAGE BG]}

Page, B. (Hrsg.), Informatik im Umweltschutz - Anwendungen und Perspektiven. Dldenbourg Verlag, München Wien 1986

\section{[PRITSKER 79]}

Pritsker, A. A. B., Compilation of Definitions of Simulation, Simulation, August 1979, S. 61-63

\section{[RETTI B2]}

Retti J., A Eramework for interactive engeneering of simulation models for time-oriented systems. In: Trappl, $R$. (Hrsg.), Cybernetics and Systems Research, North-Holland, $198 \mathrm{C}$

\section{[SCHMIDT 79]}

Schmidt, B., Simulation zeitdiskreter Systeme. Informatik Spektrum 2/79 5. 81-85 


\section{[SCS 79]}

SCS Technical Committees, Ierminology for Model Credibility. Simulation, März 1979

\section{[ZEIGLER B4]}

Zeigler, B. P., Multifacetted Modelling and Discrete Event Simulation. Academic Press, London 1984 
Interaktive Informations- und Entscheidungshilfe Systeme für Industrielles Risilko, Gefahrengut und Sonderablallmanagenent

\author{
Kurt Fedra \\ International Institute for Applied Systems (IIASA) \\ A-2361 Laxenburg, Austria
}

\title{
Kurctessune:
}

Für den Bereich grosser und komplexer Sozio-technischer- und Umweltsysteme werden interaktive, modellgestützte Entscheldungshtlfe- und Informationsysteme vorgestellt, die unter Verwendung moderner Computertechnologle Elemente des Operations Research, der Angewandten Systemanalyse und der Künstlichen Intelligenz mit problemspezifischen Datenbanken. Simulations- und Optimlerungmodellen vereinen.

Durch symbol- und graphik-orientierte Benutzerschnittstellen auf der Basis hochauflösender Farbgraphik und unter Verwendung von kontext-gesteuerter Menuetechnik wird dabei auch dem ntoht technisch ausgebildeton Anwender der unmittelbare Zugang zu wissenschaftlich-technischer Information als problemorlentlert aup berelteter Entscheldungsgrundlage ermöglicht.

Als Beisplel wird ein am IIASA in Zusammenarbeit mit EURATOM, Joint Research Centre, Ispra Establishment der Europäischen Gemeinschaft und dem Niederländischen Planungs- und Umweltministerium durchgef ührtes Projekt vorgestellt: "Advanced Dectston-ortented Software for the Manegement of" Hazardous Substances".

\section{Gliederung:}

1. Einleitung

2. Ein Anwendungsbeispiel

3. Simulations- und Optimierungsmodelle

4. Integrierte Entscheidungshilfe

5. Zusammenfassung

6. Zitierte Literatur 


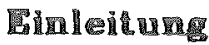

Die rasante Entwicklung der Informationstechnologie und Rechentechnik macht es in zunehmendem Masse möglich, immer anspruchsvollere Anwendungen mit aufwendigen Benutzersohnittstellen, etwa unter Verwendung von Menueteohnik, interaktiver Farbgraphik, und echtsprachlicher Ein- und Ausgabe zu ontwickeln. Damit wird es möglich, nicht nur einen wesentlich vergrösserten Benutzerkrels zu erreichen, sondern uch oine neue Bandbreite der Kommunikation zwischen Benutzer und Maschine zu realisieren.

Durch eíne entsprechend benutzerfreundliche Schnittstelle, die auch im Sinne eines Expertensystems wesentliche Komponenten anwendungsspezifischen Fachwissens beinhaltet, können nun etwa Entschoidungsträger ohne besondere technische und Computererfahrung direkten Zugang zu sehr grossen Datenvolumen oder komplexen analytischen Verfahren gewinnen (Fedra und Loucks, 1985). Durch die direkte Einbindung des "Tndbenutzers" der Information und saine (inter)aktive Mitarbeit bel deren Auswahl, Aufbereltung und Darstellung können menschliche Erfahrung und Intuition mil den Möglichkelten des Computers unmittalbar veraint werden. Damit können Entscheidungshilfesysteme geschafien werden, die sowohl Hintergrundinformation aus zahlreichen relevanten Fachgobioton und Wissensborelohen Llerern, als auch Modellrochnungen, Prognoson und Szenarioanalysen erlauben, und sohllesslich den Entscheidungsprozess selbst strukturieren helfen.

Das dem Boreich der Künstlichen Intelligenz (KI) ontnommene Modell derartiger Expertensysteme versucht direkt Strategien menschlichen Problemlöseverhaltens zu integrieren. Daneben steht aber auch ain gehr umfangreiches Instrumentarium "klassischer" algorithmischer Verfahren wie etwa der Optỉmierungsrechnung und zahlreiche ingenieurtechnische Modellrechnungen zur Verfügung, die es in einen gemeinsamen Rahmen zu integrieren gilt. Eine Beschreibung der Grundidee derartiger Hybridsysteme gibt Fedra et al., (1986).

Die Grundkonzeption des Systems sieht folgende konzeptuelle Hauptbestandtelle vor:

Die Benutzerschnitisielle, die den Zugang zu den einzelnen Systemfunktionen strukturiert, das Menuesystem steuert und Erklärungsfunktionen anbietet und die Steverung der Graphlk für Ein- und Ausgabe übornimmt.

Das Informetionsystem balnhaltet mehrere problemspezlfische Datenbanken; die Datenbanken sind über eine dialogorientierte Benutzerschnittstelle zugänglich, können aber auch von ainzelnen Simulationmodellen direkt angesprochen werden. Zwischen den einzelnen thematischen Datenbanken bestehen zahlreiche Querverbindungen, wobei ein am Bildschirm dargestellter Datensatz Quarverwoise enthalten kann, die direkt den Zugang zu weiteren Dateien erlauben. Die Querverblndung wird dabel durch einfaches Anwählen des in Frage kommenden Symbols, Texttells oder Zahlenwertes durch graphische Eingabe (Tablett oder Maus) und Fensterteohnik reallslert.

Das Modellsystem vereint mehrere Simulations- und Optimlerungsmodelle; die Auswahl der Modelle und thre speztische Konfiguration wird wleder über eln Dialogverfahren und unter Verwendung symbolischer Problembeschreibungen realisiert. Für alle Modelle stehen kontext-abhängige Standarddatensätze zur Verfügung, sodass die Problembeschroibung und Anpassung an eine bestimmte Situation in jewells relativer Form, also durch Veränderung des Standardwertes, vorgenommen werden kann. Zahlreiche Hilfsfunktionen unterstützten die Auswahl geeigneter Deskiptoren und Parameter.

Das Entscheidungshefesystem, das sowohl als in die optimierungsmodelle integrterter Bestandteil als auch als eigenes Post-Prozessorsystem zur Analyse und Auswahl bei diskreten Datensëtzen ausgeblldet ist (Zheo at al., 1985). Letzteres arlaubt die vergleichende Analyse und multi-kriterielle Auswahl von Alternativen, dle im Zuge der Szenarloanalyse mit den Komponenten des Modellsystems erzeugt wurden. 


\section{Fin Anwerdun:}

Am Internationalen Institut für Angewandte Systemanalyse (IIASA) wird in Zusammenarbeit mil der EURATOM Forschungstelle der Europäischen Gemelnschart ein entscheidungsorientiertes Sortwaresystem pür das Management von industrlellem Risiko, gefährlichen Gütern und Sonderabfällen entwlokell (Fodra, 1986; Fedra and Otway, 1986).

Das als Rahmensystem ontwickelte Softwarepaket ist als integrierter $\mathbf{S a t z}$ von modularen Bausteinen konzipiert, die die Grundlage pür jewells problemspezifische Teilsysteme bilden sollen. Ein erstes derartiges spezifisches Teilsystem wird gegenwärtig in Zusammonarboit mil dem Niederländischen Wohnbau-, Planungs- und Umwoltministerium zur Risikoabschätzung bei Erzeugung. Transport und Verarbeilung von Chlor entwickelt.

Das Rahmensystem veraint mehrere problemspezifische Datenbankan. Simulations- und Optimierungsmodelle und Entscheldungshlleveriahren in oinem interaktiven Dialogsystem mit waltgehend graphisoher Aufbereltung der Ausgabo. Abblldung 1 zeigt den erfassten Thomenkreis und die einzelnea Optonen der obersten Ebone des Dialogsystems. Entsprechend der modularen Natur des Systems wurde dabel bewusst mehr Wert auf exemplarische Lösungsansätze als auf aine vollständige Abdeckung des Problemgebiates gelegt.

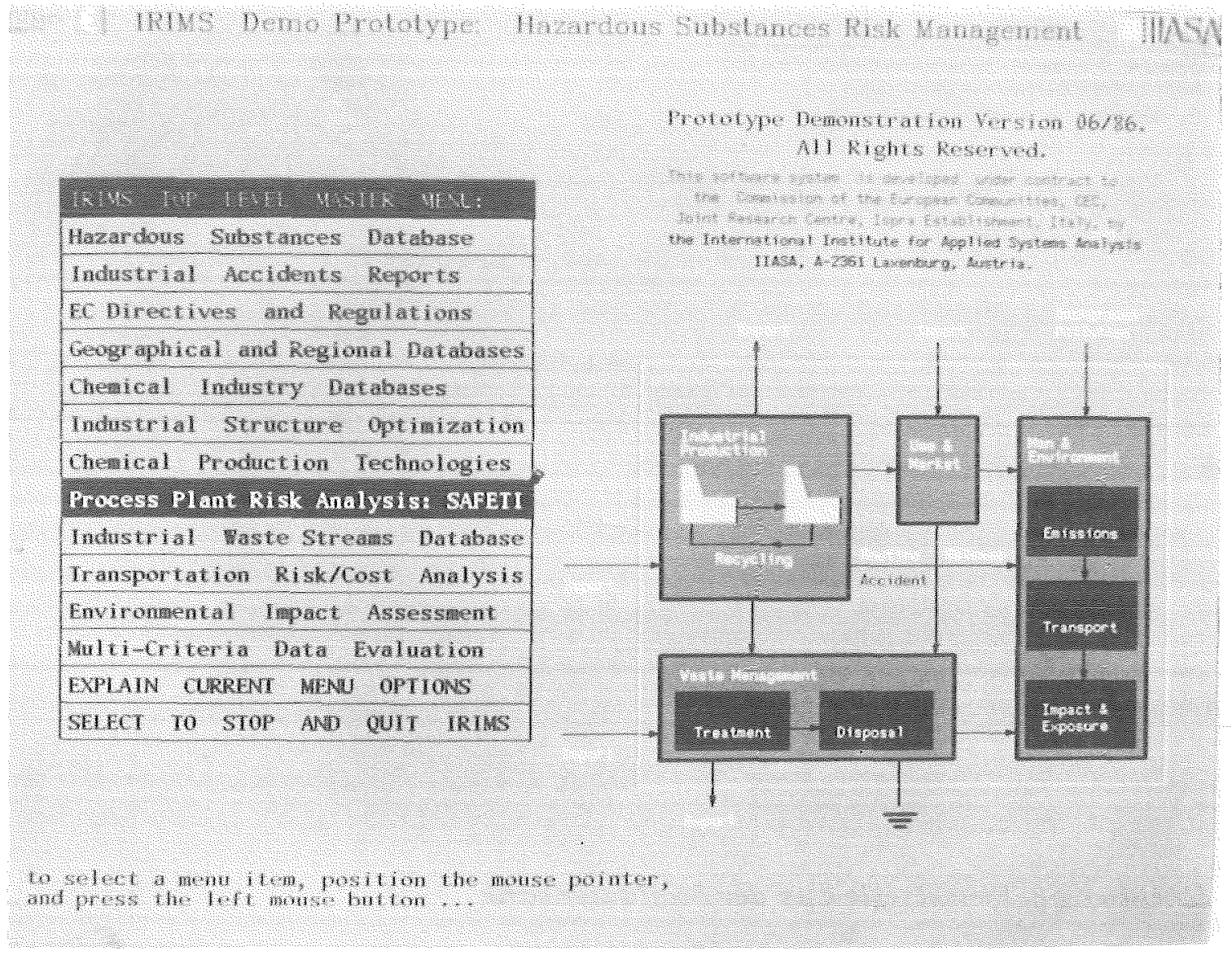

Abbildung 1: Inhalt und Struktur des Softwaresystems 
Die Datenbanken des Systems sind folgenden Themen gewldmet:

- Stoffdatenbank; sie beinhaltot neben physikalisch-chemischen und toxikologischen Kenndaten auch Produktionsinformationen und arlaubt den direkten Zugang zu welteren Datenbanken über Produktionstechnologien, Abfallströme und Gasetzestexte bzw. EG Verordnungen;

- Industrieunfëlle; beinhaltet Beschreibungen grösserer (chemischer) industrieunfälle mit Querverbindungen zur Chemikaliendatenbank und zur Datenbank industrieller Standorte.

- IG Direktiven und Gesetzestexte;

- Geographische und Regionale Datenbanken; erlauben die interaktive Generierung thematischer Karten (z.B., für Siedlungen, Industriestandorte, Verkehrswege, Flüsse und Sean, etc.) auf gesamteuropäischer bzw. regionaler Ebene.

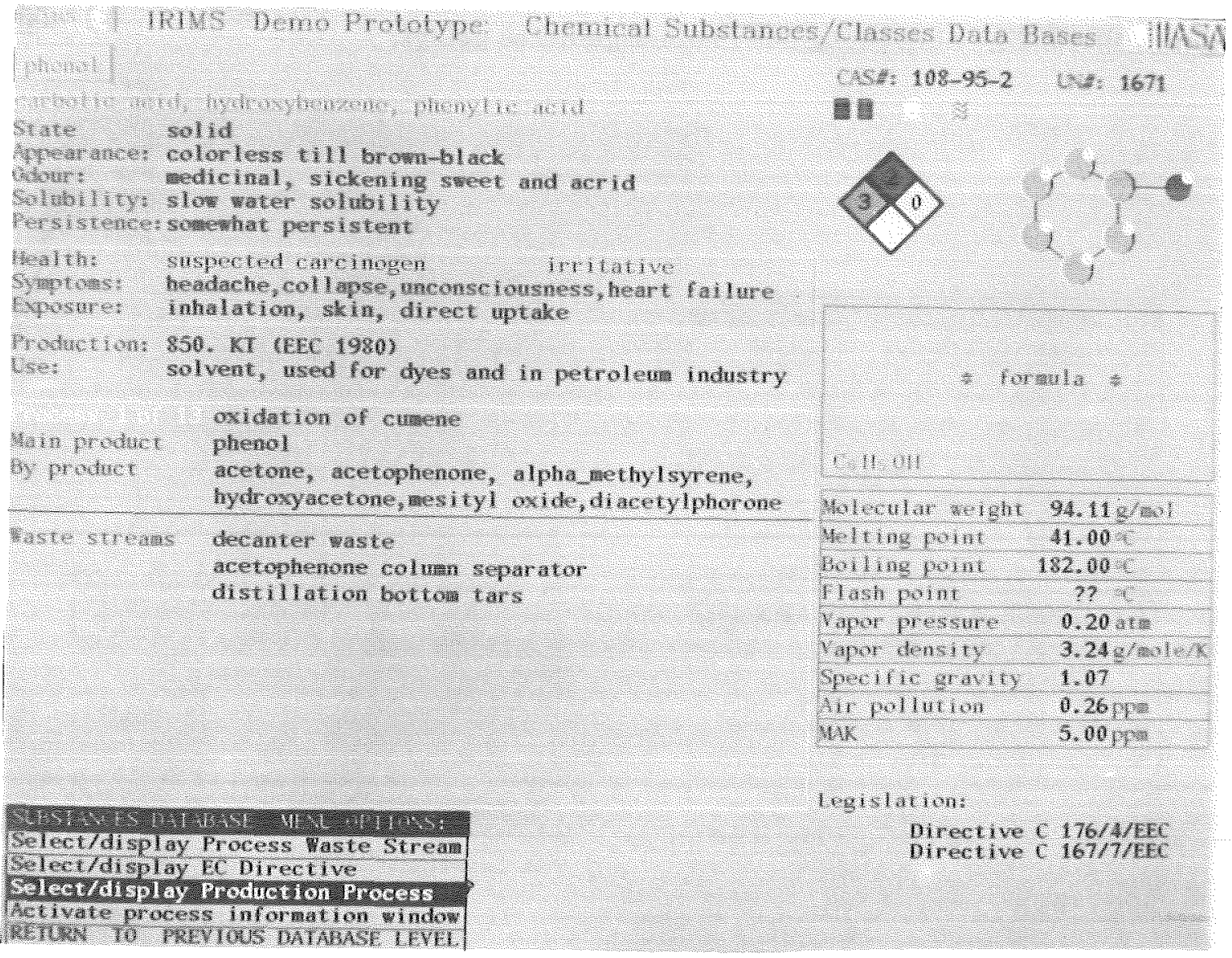

\section{Abbildung 2: Einzelseite aus der Stoffdatenbank}

- Industriestandorte; mit einer Beschreibung der wichtigsten Produktgruppen und Produkte, Produktionszahlen, und insbesondere der verwendeten bzw. gelagerten Stoffe im Sinne der EG Direktive 76/464/1, mit Querverbindungen zur Stoffdatenbank und zur Störfalldatenbank. 
- Produktionstechnologien für die in der Stoffdatenbank arfassten Stoffe, mit einer Ubersicht über Rohstoffe, Nebenprodukte and Abfallstoffe sowie einer generischen Beschrelbung einer typischen Produktlonsanlage; der Produktionsablauf kann dabei für einzelne Technologien dynamisch simullert. worden. Es bestehen direkte Verbindungen zur Stoff- and Abralldatenbank.

- Industrielle Abfälle, gruppiert nach Produktionstechnologien, Industriogruppen, Stoffgruppen und Eigenschaften, beschrelben die physikalisch-chemischen Eigenschaften der Abfallströme sowie deren wichtigste Inhaltsstoffe; mit Querverbindungen zur Stoffdatenbak und zur Standortedatenbank.

\section{Simulations und Optimierangsmodelle}

Die nächste Gruppe von Komponenten beinhaltet mehrere SImulations- und Optimierungsmodelle aus den Berelohen Produktion, Transport, Umwellauswirkungen und Risikoabschätzung.

Der Produktionsbereich ist mit einem multi-kritertellen Optimierungsmodell einer Pestizidindustrie und oinem Simulationmodell auf Prozessebene vertreten.

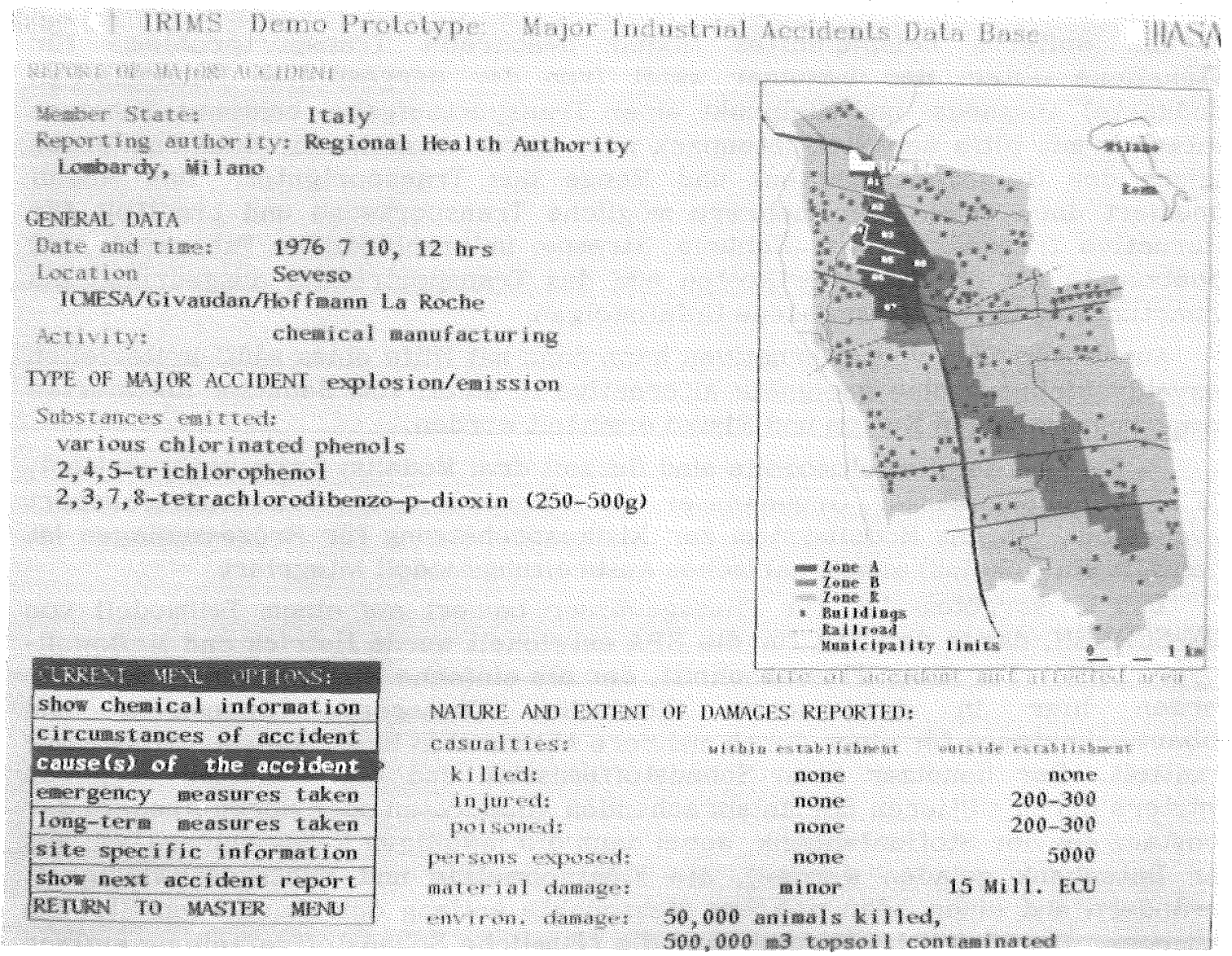

Abbildung 3: Beispiel aus der Störjalldatenbank 
Das Optimierungsmodell beschrelbt die Pestizldindustrle als technologlsches Notzwork, für das unter Berücksichtigung zahirelchor Kosten- und Umwellaktoren optimale Kapazitätsvertellungen ermittelt worden können (Fedra at al. 1982a). Der Benutzer wählt dabel im Dialogverfahren die zu minimierenden bzw. maximierenden Kriterien aus (etwa Produktlonskosten, Produktlonswert brutto und notto, Rohstof f-, Wasser- und Energleverbrauch, Abfallmenge, Abfallbeseltigungskasten), definiert Produktionsmengen für elnzelne Produkte, Preise pür Produkte und Rohstof $\mathrm{e}$, maximal zulässige Abfallmengen für bestimmte Stof

Die Lösungen werden sowohl numerisch als auch graphisch dargestellt, und in einer hlerarchischen Struktur von der Ebene der Gesamlindustrie bis zu den einzelnen Technologien bzw. Standorten mit woiteron Vorbindungen zur Stof datenbank, der Abfalldatenbank und einem Wassergütemodell zur Abschätzung der Umweltauswirkungen versohiedener Produkt.lonsst,rateglen.

Das Simulationsmodell Pür Einzelprozesse ist zur Zalt pür eine Phenolchlorierungsanlage implementiert. Das Modell verwendet eine in LISP implementierte symbolische Simulationsform, die von einer objekt-orientierten und regelbasierten Darstellungsform ausgeht und sinfaches forward chaining zur Abarbellung der Produktionsregeln verwandet.

Parallal zu diesom symbolischen Simulationsmodell ist eine Oplimierungsmodell implomentiert, das neben ökonomischen auch Risiko- und Umweltaspekte chemischer Produktlonstechnologien berücksichtigt (Graver und Fedra, 1986).

Ein welteres belsplelhaptes Modell besohralbt Transportprobleme pür gefährliche Güter: der Benutzer wählt (von der dargestellten Europäischen Landkarte) Ausgangs- und Zlelpunkt einer Transportaufgabe, bestimmt Substanz (entweder mil HLlP der Stoffdatenbank oder durch Auswahl der entsprechenden Indizes des Gofahrendlamanten) und Menge des Transportgutes. Das Modell generiert denn einen oder mehrere mögliche Transportwege und ermittell pür alternative Transportformen (Schlene. Strasse, unterschiadliche Fahrzeugtypen) Schätzwerte für die Transportkosten und das Transportrisiko, ausgedrückt als Erwartungswerte für verschledene Unfallsfolgen.

Aus diesem Satz von Alternativen kann dann mil Hilfe elnes multi-kriteriellen Auswahlverfahrens oine geelgnete Alternative in einem vom Benutzer definierten Kompromiss zwischen Kosten und Risiko ermiltelt werden.

Im Bereich der Umweltmodelle sind derzelt drel Modellsysteme implementiert, dle Ober?lächengewässer, Grundwasser und atmosphärischen Schadstof ftransport beschrelben. In das Modellsystem zur Risikoabschätzung Pür Prozessanlagen ist ebenfalls eln (lokales) atmosphärisches Ausbreitungsmodell integriert.

Das Wassergibumodell für Fliessgewăsser basiert auf ainem Teilmodell von TOX-SCREEN, das in den USA für die EPA entwickelt wurde Hetrick and McDowellBoyer, 1984). Für einen Flussabsohnilt, der als elnfache Rinne beschrieben wird, werden hier in dynamischer Simulation Transport-, Diffusions- und Abbaumechanismen für einen durch mehrere Abbauraten beschriebenen Sohadstoff ermittelt. Der Benutzer kann Schadstoffemission und Eigenschaften vorgeben (letztere durch editleren der entsprechenden Abbauraten oder durch Auswahl der Substanz aus der Stoffdatenbank, wobel dann die Jeweiligen Raten automalisch aus der Datenbank geladen werden), die Flussgeometrie und Fliessgeschwindigkeit verändern und einen oder mehrere Messpunkte entlang der simullerten Strecke festlegen. Das Modell beschrelbt dann die råumliche Schadstoffvertellung entlang der Strecke für einen Jahresgang des Abflusses in dynamischer graphischer Darstellung (Abbildung A) und registriert mittlere und maximale Schadstoffkonzentrationen an den Messpunkten. Duroh Festlegung zulässiger 
Obergrenzen der Schadstoffkonzentration und die automatische Festlegung der Schadstoffmengen (etwa aus dem obon erwähnten Modell industrieller Produktion) kann damit für eine bestimmte Produktions- oder Abfallbeseitigungsstrategie eine schnelle und einfache, wonn auch nur annähernde Uborprüfung threr Umweltverträglichkeit vorgenomen werden.

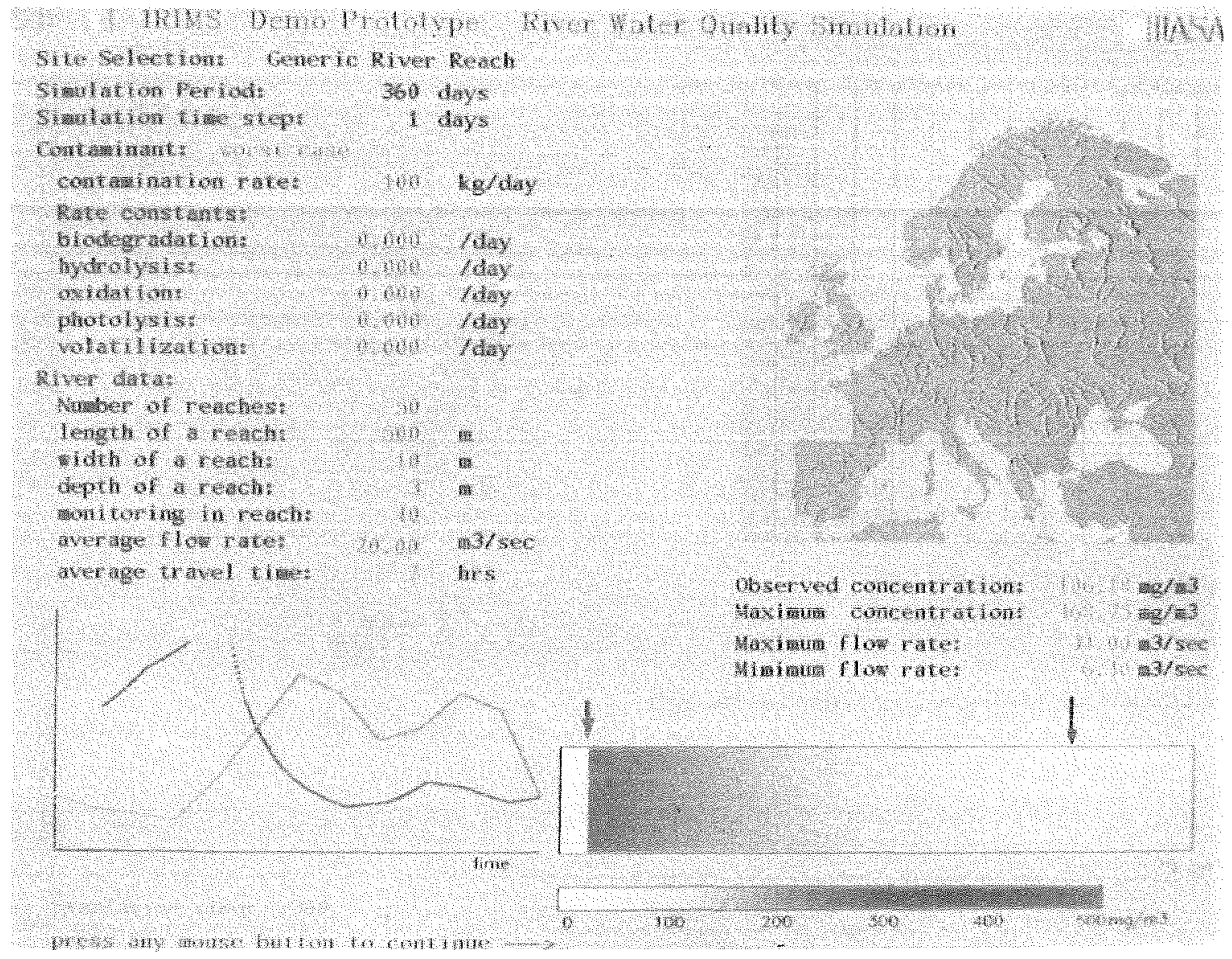

Abbildung 4: Flusswassergütemodell

Das Grundwassermodell verwendet eine etwas andere Strategie der Problemdarstellung. Ausgehend von einer Bibliothek von gegenwärtig 14 generischen Problemsituationen kann der Benutzer nach Auswahl eines dieser Datensätze eino Anpassung an die jeweilige spezifische Problemsituation durch Verändern der entsprechenden Modellparameter erreichen. Da dabei von einer kompletten Problembeschrelbung ausgegangen werden kann, können die erforderlichen Veränderungen relativ beschrieben werden, was die Verwondung des Modells wesentlich erleichtert.

Das Basismodell ist ein zweidimensionales finite Elemente Modell (Fedra at al., 1986c, Diersch und Kaden 1984), das für horizontale oder vertikale Stofftransportaufgaben konfiguriert werden kann (Abblldung 5). Dabel werden insbesondere Kontroll und Sanierungsmassnahmen wie hydraulische oder mechanische Barrieren berücksichtigt. 


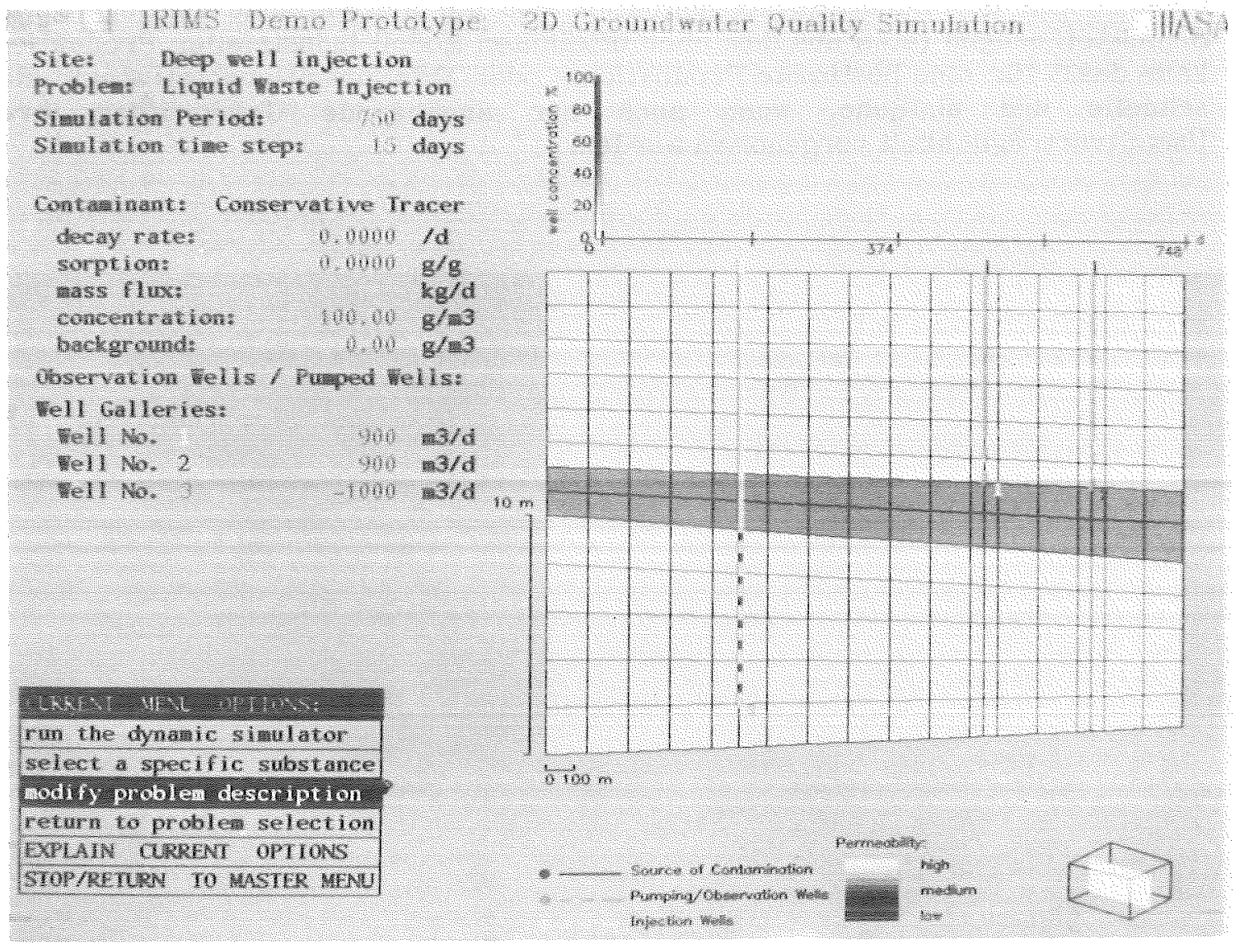

Abbildung 5: Grundwassergütemodell

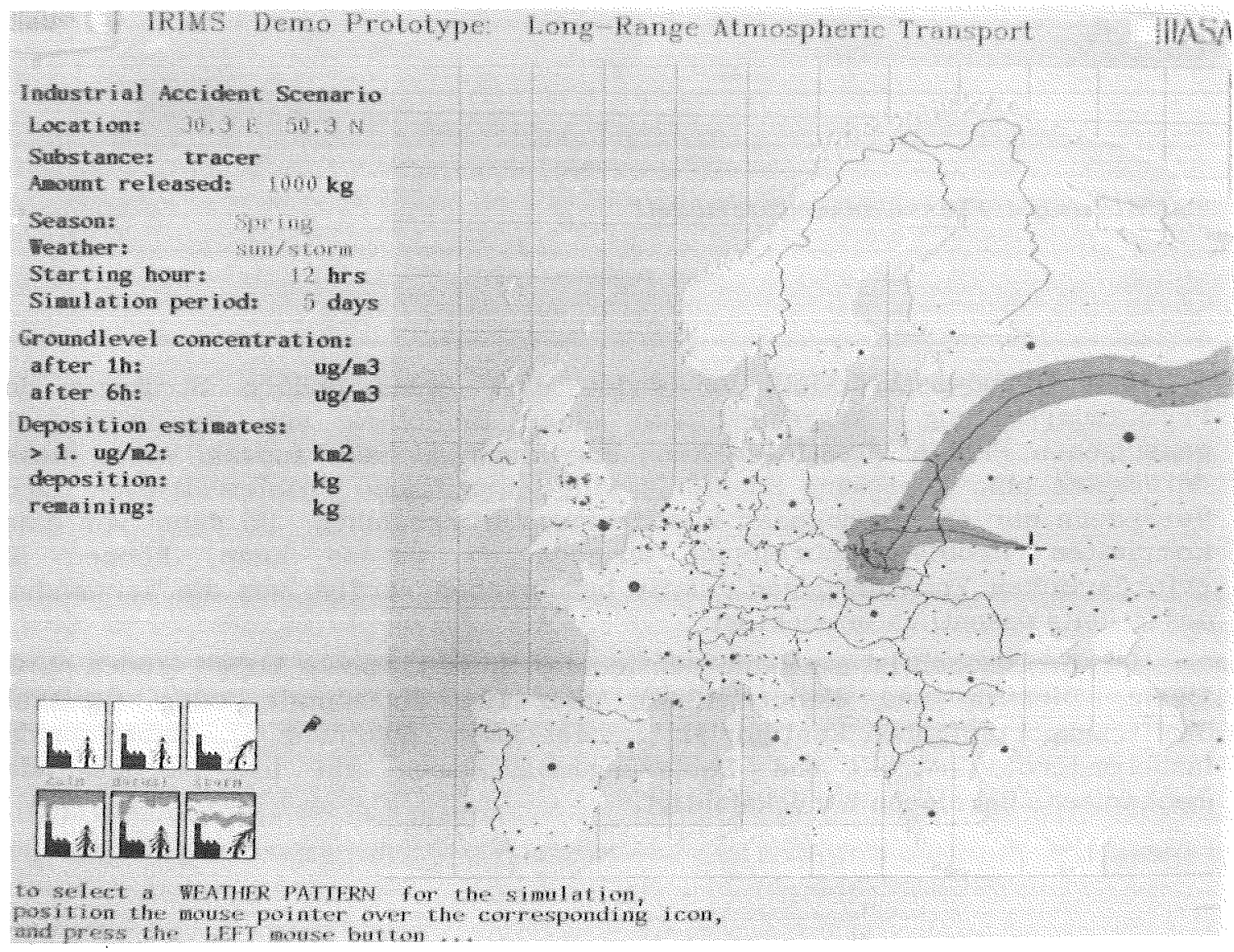


Für den überregionalen Transport atmosphärischer Schadstoffe, atwa nach einem grossen Industrieunfall, ist eln welteres Simulationsmodell integriert. Der Benutzer kann nebon Sohadstof fmonge und -art den Ort. die Tages- und Jahreszelt, sowie die Wetterbedingungen am Unfallort interaktiv festlogen. Dabel werden, etwa im Falle der Wellerbedingungen, symbolischo Darstellungen typischer Wettersituationen angeboten (Abildung 6). Die resultierenden Modellparameter, ebenso wle die für die dynamische Simulation benöligten Winddaten werden automatisch aus den entsprechenden Datenbanken des Systems geladen. Die Modelläufe zeigen dann den Verlauf der Trajektorian und der Ausbrellung der Schadstoffemission über einer europäischen Landkarle und berechnen bodennahe Konzentrationen und Depositionswerte.

Zur Risikoabschätzung für Chemieanlagen schliesslich wird an einer Intogration des Softwarepaketes SAFRTI (Tochnica, 1984) gearbeltet. Dleses Modellsystem sohätzt Anlagenrisiken auf der Basis von Fehlerbaumanalyse und nachfolgender Ausbreitungsrechnung von Schadstoffen unter Berücksichtigung der lokalen Bevölkerungsverteilung.

\section{Integrierte Fotscheidunghilfe}

Neben der Informationsfunktion der Datenbanken und Simulationsmodelle, die spezifisohe Handlungsalternativen zu beschreiben erlauben, sind explizite Entscheldungshilf efunktionen in den Optimierungsmodellen und in oinem multkriteriellem Selektionsmodul vorhanden. Die im System integrierten interaktiven Optimierungsmodelle erlauben dem Benutzer, Auswahlkriterien festzulegen, zulässige Bereiche für einzelne Varlable festzuschreibon, und sohliesslich duroh die Vorgabe von "Wunschvorstellungen" eine implizite Gewichtung der einzelnen Kriterien vorzunehmen. Ein als Post-prozessor ausgeblldates Teilsystem zur diskreten Optimierung oder Auswahl aus einer Menge von Alternativen unterstützi eine gleicharlige Problemformulierung (Majchrzak, 1984; Zhao et al., 1985). Die von den einzelnen Modellen des Systems generierten Alternativen können mit Hilfe des Auswahlsystems vergleichend dargestellt werden, otwa durch paarweisen Verglelch von Einzelkriterien. Der Entscheldungsraum kann durch sukzessive Elimination von ungeeigneten Alternativen zunehmend überschaubarer gemacht werden, bis denn einfache Kompromisslösungen gefunden werden können. Wesentlich ist dabei, dass weitore Hintergrundinformation aus den Simulationsergebnissen oder den Datenbanken zur Unterstützung des Entscheidungsprozesses beigezogen werden kann.

\section{2usammenfagsun}

Die Intogration unterschiedlicher Problemdarstellungen und Analyseverfahren in elnem einheillichen, durch entsprechende symbolische und besonders graphische Benutzerschnittstellen leloht zugänglichem System und die damit mögliche direkte Einbindung des Endbenutzers der Information in deren Verarbeitungs- und Darstellungsprozess bletet insbesondere für komplexe Systeme und Problemsituationen, für die eine vollständige formale Beschrelbung nicht sinnvoll erscheint, altraktive Alternativen zu klassischen Ansätzen des Operations Research und der Angewandten Systemanalyse. Die hohe Flexibllitül modularer und hybrider Systeme und die Entwicklungsphilosophie des rapid prototyping erlauben elne sehr problemorientierte und adaptive Vorgangswelse, die zu einer wesentlich verbesserten Akzeptanz computerunterstützter Methoden Pühren sollte. 


\section{0.}

Diersch, H.-J, and Kaden, S. (1984) Contaminant Plume Migration in an Aquifer. Finlte Element Modeling for the Analysis of Remediation Strategles: A Case Study. CP-84-11, International Institute for Applied Systems Analysis, A-2361 Laxenburg, Austria, 68pp.

Fedra,K. and Loucks,D.P. (1985) Interactive Computer Technology for Planning and Policy Modeling. Water Resources Research, 21/2, 114-122.

Fedra, K., Karhu, M., Rys, T., Skooz, M., Zebrowski, M. and Zlembla, W. (1986) Model-based Decision Support for Industry-Environment Interactions. A Pesticide Industry Example. CP-86-8x. International Institute for Applied Systems Analysis. A-2361 Lasenburg, Austrla. Forthcoming.

Fedra,K., Welgkricht,E., and Winkelbaver,L. (1986) A Hybrid-Approsch to Information and Decston Support Systems: Hazardous Substances and Industrial Risk Management. Paper presented at the first Conierence on Economios and Artificlal Intelligence, Alx-en-Provence, 2-4 September. Pargamon, forthooming.

Fedra,K. and Otway,H. (1986) Advanced Declsion-oriented Software for the Management of Hazardous Substances. Part III. Deolston Support and Expert Systems: Uses and Users. CP-86-14, International Institute for Applied Systems Analysis, A-2361 Laxenburg, Austria. 44p.

Fedra,K. (1986) Advanced Decision-oriented Software for the Management of Hazardous Substanoes. Part II. A Demonstration Prototype System. CP-86-10, International Institute for Applied Systems Analysis, A-2361 Laxenburg. Austria. 98p.

Fodra, K. (1984) Systemsimulation als Entschoidungshllfo im Umweltbereich. (Proceedings of ASIM 84, 2. Symposium Simulationstechnik, Wien, 25-27 September 1984, Springer Heidelberg.)

Graver,M. and Fedra,K. (1986) Intelligent Decision Support for Technology Assessment: The Chemical Process Industry. Presented at the VIIth International Conference on Multiple Criteria Decision Making. 18-22 August, 1986, Kyoto, Japan.

Heirick, D.M. and MoDowell-Boyer, L.M. (1984) User's Manual for TOX-SCREEN. A mult-medie screening level progrem for assessing the potential fate of chemicals released to the onvironment. ORNL-B041. Oak Ridge National Laboratory and EPA-560/5-83-024, USEPA. Washington D.C., 301p.

Majchrzak. J. (1984) Package DISCRETE for multicriteria optimization and decision making problems with discrete alternativas. IIASA conference on Plural Rationality and Interaotive Decision Processes, 16-20 Augusi, 1984, Sopron, Hungary.

Technica (1984) The SAFETI Package. Computer-based System for Risk Analysis of Process Plant. Vol I-IV. Technica Ltd., Tavistook Sq., London.

Zheo, Ch., Winkelbauer, L. and Fedra, K. (1985) Advanced Decision-oriented Software for the Management of Hazardous Substances. Part VI: The Interactive Decision Support Module. 


\section{WissensBasierTe Simulation eines ÖKOSVSTEMS}

S. Becker, G. Hille, H. Ramlow

Fachbereich Informatik der Universitat Hamburg

\section{Zusammenfassung:}

Dieser Beitrag beschreibt die Realisierung der Kopplung eines Expertensystems mit einem Simulationsprogramm. Die aus dem Simulationsprogramin gewonnenen Daten werden in eine abstraktere Darstellungsweise transferiert, ein Regelsysten arbeitet auf diesen Daten und steuert die Simulation. Das System wird zum Auffinden von erwünschten Zuständen des modellierten Systems verwendet. Erste Ergebnisse einer wissensbasierten Simulation werden vorgestel1t und diskutiert.

\section{G1 iederung}

1. Einleitung

2. Beschreibung des Anwendungsbereichs

3. Simulation und Expertensysteme

4. Zielsetzung

5. Anforderungen an das System und deren Realisierung

6. Systemarchitektur

7. Zur Problematik einer wissensbasierten Simulation

8. Ausblick für Weiterentwicklungen

9. Literaturverzeichnis 


\section{Einleitung}

Bei dem hier vorgestellten System handelt es sich um einen wissensbasierten Ansatz zur Steuerung eines Simulationsprogrames und zur Optimierung des Sucherfolges mittels Graphsuche.

Das Expertensystem besteht aus einer Kopplung eines traditionellen, quantitativen, kontinuierlichen Simulationsmodells mit einem auf vagem, qualitativen Wissen beruhenden Expertensystem.

A1s Anwendungsbeispiel für die Simulationskomponente wurde die in der Simulationsliteratur bekannte Eutrophierung eines Sees gewähit /AND73/. Das hier konzipierte Vorgehen kann jedoch prinzipiell auch auf andere Simulationsmodelle übertragen werden.

Das Simulationsprogramm ist in einer "konventionellen" Programiersprache (PASCAL) erstellt worden, für die wissensbasierte Komponente ist die KI-Sprache OPS5 verwendet worden /BR085, FOR81, HAY83/.

\section{Beschreibung des Anwendungsbereichs}

\section{Das Seemode11}

Ein see ist ein sehr komplexes und inhomogenes ökologisches system, das im folgenden nur für das weitere Verständnis beschrieben wird.

Die Hassertemperatur in einem See nimnt im allgemeinen mit der Wassertiefe ab. Die obere, wärmere Wasserschicht wird als Epilimnion, die untere als Hypolimnion bezeichnet. Im Somener findet kein Stofraustausch zwischen den beiden Schichten statt, im Herbst und Frühling wird das Wasser jedoch durchmischt. Wegen des Lichtbedarfs spielt sich das pflanzliche Leben nahe der ober.. fläche ab. Das Wachstum ist durch die vorhandenen Nährstoffe begrenzt. Abgestorbene Pflanzenteile sinken in das Hypolimnion und bilden den Detritus. Dieser zersetzt sich unter Sauerstoffverbrauch und gibt dabei in sich gebundene Nährstoffe wieder frei. Eine Überdüngung des Sees durch Pflanzennährstoffe hat ein starkes Anwachsen der Biomasse und damit einen wachsenden Anfall von Detritus zur Folge. Ist der Sauerstoff des Hypolimnions nicht ausreichend zu dessen Zersetzung, so wird durch die Bildung von Faulschlamm das höhere Leben im See vergiftet.

Man unterscheidet zwischen oligotrophen (nährstoffarmen) und eutrophen (nährstoffreichen) Seen. Der übergang vom oligotrophen zum eutrophen Zustand wird als Eutrophierung bezeichnet und ist ein natürlicher Vorgang, er dauert im allgemeinen Jahrtausende, kann sich aber durch Zivilisationsfolgen (Abwässer, Landwirtschaft) innerhalb weniger Jahre vollziehen. Diese beschleunigte Eutrophierung gilt es zu vermeiden.

Anderson beschreibt ein Simulationsmode11, mit dem verschiedene 
Möglichkeiten zur künstlichen Eutrophierung untersucht werden können. Folgende Größen werden in seinem Model1 bericksichtigt:

- Nährstoffzufuhr

- Nährstoffimenge in See

- Biomasse im See

- Detritusmenge im See

- Sauerstoffmenge im See

A1s mögliche Maßnahmen gegen die Eutrophierung betrachtet er:

- Einsatz von Algiziden

- Abernten von Biomasse

- Ausbaggern des Detritus

- Belüften, des Hypolimnions

Aus biologischer Sicht ist dieses Modell viel zu ungenau, um hieraus konkrete Aussagen über das tatsächliche Verhalten eines Sees zu machen, weil

- der Sauerstoffgehalt des Epilimnions als konstant angesehen wird

- die Trennung und Durchmischung der Schichten nicht ausreichend modelliert ist

- die Biomasse nicht in die einzelnen Arten getrennt wird

- Kohlenstoff als einziger Nährstoff betrachtet wird

- die Maßnahmen unerwinschte, nicht modellierte Nebeneffekte zeigen

Für die Untersuchungen am wissensbasierten System wurde dennoch dieses Model1 gewäh1t. Hierdurch ergeben sich Vergleichsmöglichkeiten zwischen Andersons Ergebnissen und dem Simulationsergebnis einer wissensbasierten Simulation. Das hier konzipierte wissensbasierte Vorgehen läBt sich prinzipiell auch auf bessere Modelle übertragen.

\section{Simulation und Expertensysteme}

Die Methode der Simulation ist zur Lösungsfindung bei realen Problemen sehr verbreitet. Dies gilt insbesondere dann, wenn das reale Problem derart komplex ist, dab mathematische Verfahren nicht anwendbar sind. Dies ist gewöhnlich bei ökologischen Fragestellungen der Fall. Eine Validierung des Modells ist wegen seiner Komplexität zumeist nicht möglich. Vergleiche zwischen Simulationsergebnissen und Messungen am realen System können Hinweise auf die Güte des Modells geben.

Die Veränderung von Modellparametern zur optimierung von Zustandsvariablen oder zur 0ptimierung des Gesamtzustandes des Systems wird durch das Simulationsprogramm ermöglicht. Aufgrund der Komplexität des Mode11s und der mit der Zahl der Parameter exponentiel1 steigenden Größe des Suchraums ist das Auffinden einer Lösung schwierig oder gar unmöglich.

Zur Lösungsfindung (oder auch zur Modellverbesserung) wird deshalb ein Experte benötigt, der das Verhalten des realen Systems 
kennt und aufgrund seines Expertenwissens gezieltere Parameteränderungen vornimmt und damit den Suchraum einschränkt.

So erscheint es naheliegend, Expertenwissen und Simulationmodelle miteinander zu verbinden /SHA85/. Das Expertenwissen kann in verschiedene Bereiche des kombinierten Systems eingebracht werden:

- qualitative Beurteilungen

Z.B.: der Nährstoffgeha1t ist kritisch hoch, wenn...

- qualitative Vergleiche

Z.B.: Belüften ist immer besser als Algizidzugabe

- Domänenwissen

Z.B.: Diese MaBnahne führt nicht Zu...

- Strukturierung des Suchraumes

Ż.B.: Jetzt sollte die MaBnahme (x) weiter angewendet werden, anstatt...

- Begründen

z.B.: Dies führt zu ..., weil ...

Als Vorteile einer Kombination aus Simulationsprogramin und Expertensystem ergeben sich:

- Beschränkung des Suchraums in Komplexität und GröBe

- Strukturierung des Suchraums

-Strategieauswahl bei der Suche

- Fähigkeit zum Lernen

- Validierung der Expertenentscheidungen

\section{Zielsetzung}

Das Ziel der Arbeit ist, ein wissensbasiertes System zur Steuerung eines (gegebenen) Simulationsmodells zu konstruieren. Anhand des gewählten Beispiels bedeutet dies konkret die überwachung und Beeinflussung des Gesundheitszustandes eines (simulierten) Sees über ein Zeitinterval1 hinweg.

Das entstandene System soll dazu dienen, einen Benutzer (Biologen/Wasserwirtschaftler) bei einer Abschätzung der Entwicklung eines Sees und der Suche nach eventuell notwendigen, effektiven GegenmaBnahmen zu unterstützen. Damit dies möglich ist, muß der Benutzer in der Lage sein, dem System die Ausgangssituation des Sees mitzuteilen, eine qualitative Beurteilung für Werte der relevanten Zustandsvariablen des Sees vorzunehmen, die Dosierung von möglichen Gegenmaßnahmen zu beurteilen und den System heuristisches Wissen über Gründe fuer ihre Anwendung einzu-geben. Er muß also über ausreichendes Domänenwissen verfügen.

Diese Angaben erlauben dem System, problemangepaBte Lösungen zu 
finden. Darunter sind solche Lösungen zu verstehen, die aufgrund der Stärke und der Art der verwendeten MaBnahmen wünschenswert erscheinen. Gefundene Lösungen sollen das vom Benutzer definierte Ziel (normale Werte zu erreichen) möglichst genau treffen.

\section{Anforderungen und deren Realisierung}

Der Suchraum für eine optimale Einstellung der Maßnahmenparameter ist sehr groB. Das liegt zum einen an der bereits erwähnten Kombinatorik dieser Parameter, zum anderen an der Kontinuität ihrer Wertebereiche. Um in absehbarer Zeit die Lösungssuche beenden zu können, ist es nötig, den Suchraum einzuschränken.

Durch Abstraktion der quantitativen Werte der Maßnahmenparameter auf qualitative Beschreibungen wie z. B. "schwach" oder "sehr stark" wird eine Diskretisierung vorgenomen, die auch Nichtlinearitäten des jeweiligen Wertebereichs berücksichtigen muB. Dies erfordert Expertenwissen. Ebenso wer den auch die Wertebereiche der Zustandsvariablen abstrahiert. Für sie sind Beurteilungen wie "normal" oder "kritisch hoch" vorgesehen. Der Gesamtzustand des Sees wird dann als "normal" betrachtet, wenn alle Zustandsvariablen mit "normal" bewertet sind.

Vorteil eines solchen Verfahrens ist nicht nur die Reduktion des Suchraumes durch Vergröberung. Vielmehr ist es durch die qualitative Beurteilung der Werte der Zustandsvariablen überhaupt erst möglich, ihre Bedeutung für den einzelnen See angemessen zu beschreiben. Beispielsweise mag dieselbe Nährstoffkonzentration $x$ für einen Flachlandsee "normal" sein, für einen Alpensee aber bereits "hoch". Anders ausgedrückt: Soll aufgrund von MeBwerten entschieden werden, ob Maßnahmen ergriffen werden müssen, so hängt dies nicht so sehr von deren Höhe, als vielmehr von ihrer Bedeutung für den speziellen See ab. Hierdurch wird die Grundlage für eine Strukturierung und damit für eine wesentliche Reduktion des Suchraumes gegeben. Eingeführt werden die Prinzipien der Indikationen, Kontraindikationen und Grenzen.

Ein Maßnahmentupel ist die Zusammenfassung mehrerer MaBnahmen mit den jeweils zugehörigen Stärken der MaBnahme. Eine Indikation ist ein Tripel (Z1, B1, M), das besagt, daB das zuletzt simulierte Maßnahmentupel um die Maßnahme M erweitert, bzw. die MaBnahme $M$ in inm verstärkt werden muB, falls Zustand $Z 1$ mit B1 bewertet ist. In den Indikationen wird also Wissen über die Gründe für die Anwendung einer bestimnten MaBnahme modelliert .

Mit einer MaBnahme $m$ sind $u . U$. aber auch negative Auswirkungen auf andere Zustandsvariablen verbunden. Dann gilt es diese durch Restriktionen für ihre Anwendung zu vermeiden. Dieses Wissen wird in den Kontraindikationen und Grenzen modelliert.

Eine Kontraindikation ist ein Tripel (Z2, B2, M), das die Anwendung der Maßnahme M verbietet, falls die Zustandsvariable $\mathrm{Z2}$ mit B2 bewertet ist. Annlich eine Grenze: Sie ist ein Quadrupel IZ3, 
B3. M. D), das die Anwendung der MaBnahme Mur in höchstens der Dosierung D ZuläBt, ralls Zustandsvariable Z3 mit B3 bewertet ist. Ein Vorschlag für eine Maßnahme kann also dann aufgegriffen werden, wenn mindestens eine Indikation für und keine Kontraindikation gegen sie spricht, und sie keine Grenze verletzt.

Für die Erzeugung eines MaBnahmentupels aus mehreren Einzelmaßnahmen ist das so modellierte Wissen noch nicht ausreichend. Nicht geklärt ist, wie die MaBnahmen zusammenwirken und in welcher Stärke sie angewendet werden sollen. Dieses Problem wird mittels Graphsuchverfahren gelöst. Wird durch die Simulation eines Maßnahmentupels ein Zustand des Sees erreicht, der nicht "norma 1" ist, und werden aufgrund dessen $n$ verschiedene MaBnahmen vorgeschlagen, so werden $n$ neue Maßnahmentupel erzeugt, die jeweils un genau eine PaBnahme gegenüber dem alten erweitert sind, bzw. in denen jeweils genau eine MaBnahme um eine Stufe verstärkt wurde. Diese neuen MaBnahmentupel werden wieder simuliert und erweitert, bis eine Lösung gefunden wurde, der Benutzer kann dann weitere suchen lassen, oder bis keines der MaBnahmentupel mehr erweiterbar ist. Dies bedeutet, daß für die gestellte Aufgabe keine weiteren Lösungen existieren. Gestartet wird mit einem MaBnahmentupel, in den keine Maßnahmen vorgeschlagen werden.

Für das Graphsuchverfahren wurden verschiedenen Strategien (Heuristiken) bereitgestellt, die eine effiziente Suche problemangepaßter Lösungen ermöglichen sollen. Inwiefern sie dies leisten, ist noch Gegenstand der Untersuchung, wenn auch erste, positive Ergebnisse bereits vorliegen. Die Strategien bieten verschiedene Möglichkeiten für die Auswahl des nächsten zu simulierenden Maßnahmentupe 15 . So $z$. B. die Bevorzugung möglichst schwach dosierter Maßnahmen, die Bevorzugung bestimmter Maßnahmen (deren Kosten gering sind) oder die Wahl eines MaBnahmentupels, das der Lösung wahrscheinlich schon recht nahe komnt. Eine genauere Beschreibung der Strategien soll an dieser stelle nicht erfolgen.

Zusamengefaßt leistet das System folgendes:

Es interpretiert Simulationsergebnisse auf die Erfüllung des gesteckten Zieles oder auf Verbesserungsvorschläge der zugrundeliegenden Maßnahmentupel hin. Der Suchraum für Lösungen, wird durch den Einsatz von Expertenwissen reduziert, die Suche sol1 durch Heuristiken effizient gehalten werden. Unlösbare Aufgaben werden erkannt, bedeuten aber einen hohen Suchaufwand.

Sofern die oben beschriebenen Methoden auf andere Domänen anwendbar sind, ist es möglich, das hier konzipierte Vorgehen prinzipiell auf sie zu übertragen und die sich dort stellenden Probleme mit einem analogen System zu behandeln. 


\section{Systemarchitektur}

Das wissensbasierte Simulationssystem besteht aus den drei Komponenten:

- Simulationsprogramm

- Benutzeroberfläche

- Wissensbasiertes Regelsystem

Das Simulationsprogramm enthält in erster Linie das Simulationsmode11. Dazu koment eine eigene, einfache Benutzerschnittstelle, mittels derer modellspezifische Grundwerte eingestellt werden können. Da das Simulationsmodell aus Effizienzgründen in PASCAL realisiert wurde, ist eine Schnittstelle für die Kommunikation zwischen inm und der wissensbasierten Komponente nötig. Ansonsten ist die Simulationskomponente völlig unabhängig vom restlichen System und soll hier als "black box" betrachtet werden.

Die Benutzeroberfläche besteht zum einen Teil aus Regeln, die dem Benutzer Informationen über den bisherigen Ablauf liefern. Unerwünschte Informationen können dabei durch Angabe der gewünschten Ausführlichkeit unterdrückt werden.

Zum anderen Teil enthält die Benutzeroberfläche auch Regeln, die solche Interaktionen des Benutzers ermöglichen, die den weiteren Programmablauf beeinflussen. Insbesondere sind dies:

- Veränderung der Vorschläge für anzuwendende Maßnahmen im dynamischen MaBnahmenpool

- Veränderung des Zeitpunktes des Maßnahmeneinsatzes

- Festlegen einer Bewertungsstrategie für erzeugte Maßnahmen

- Veränderungen an der statischen Vissensbasis

Die wissensbasierte Komponente stellt das Kernstück des Systems dar. Inre Unterkomponenten sollen hier in der dem Ablauf des Programmes entsprechenden Reihenfolge betrachtet werden.

Im einzelnen hande1t es sich dabei um (siehe Abb. 1):

- Simulationssteuerung

- Beurteilungskomponente

- Maßnahmengenerator

- Begründungskomponente

- Strategien zur Maßnahmenbewertung

- dynamisches Maßnahmenpool

- statische Wissensbasis

Die Simulationssteuerung sorgt für die notwendige Parameterversorgung des Simulationsmodells. Insbesondere greift sie dazu den jeweils relevantesten Vorschlag für anzuwendende Maßnahmen aus dem dynamischen MaBnahmenpool heraus. Ansch1ießend startet sie das Simulationsprogramm. 


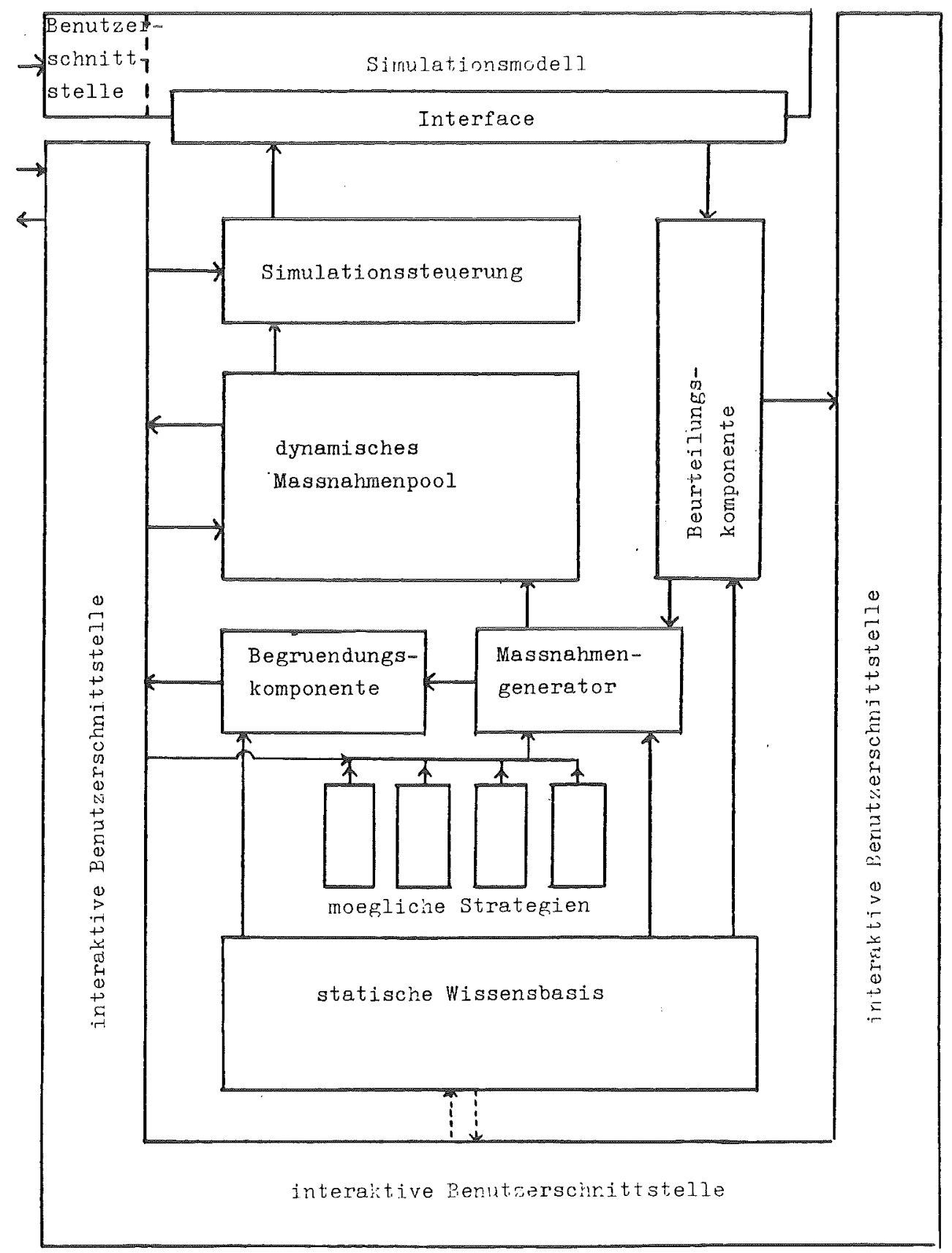

Abb.1: Struktur des Gesamtsystems

Die Beurteilungskomponente bewertet aufgrund von Wissen aus der statischen Wissensbasis das erzielte Simulationsergebnis. Das Ergebnis und dessen Bewertung werden an die Benutzeroberfiache weitergegeben. Im positiven Falle handelt es sich um eine Lösung des Problems. Im negativen Falle werden die Bewertung und der zuletzt simulierte Vorschlag für anzuwendende MaBnahmen an den Maßnahmengenerator weitergegeben. 
Der Maßnahmengenerator versucht den zuletzt simulierten, nicht erfolgreichen Vorschlag für anzuwendende Maßnahmen um erfolgversprechende EinzelmaBnahmen zu erweitern. Dazu wird heuristisches Wissen aus der statischen Wissenbasis verwendet. Die neu entstandenen Vorschläge werden aufgrund der gewählten Strategie in ihrer Relevanz bewertet und dem dynamischen MaBnahmenpool beigefügt, sofern sie nicht bereits simuliert wurden. Die Erzeugung von Begründungen für diese Vorschläge wird angestoßen.

Die Begründungskomponente erzeugt für alle Vorschläge des MaBnahmengenerators für oder gegen neue MaBnahmen eine ausführliche Begründung, die dann an die Benutzeroberfläche weitergegeben wird.

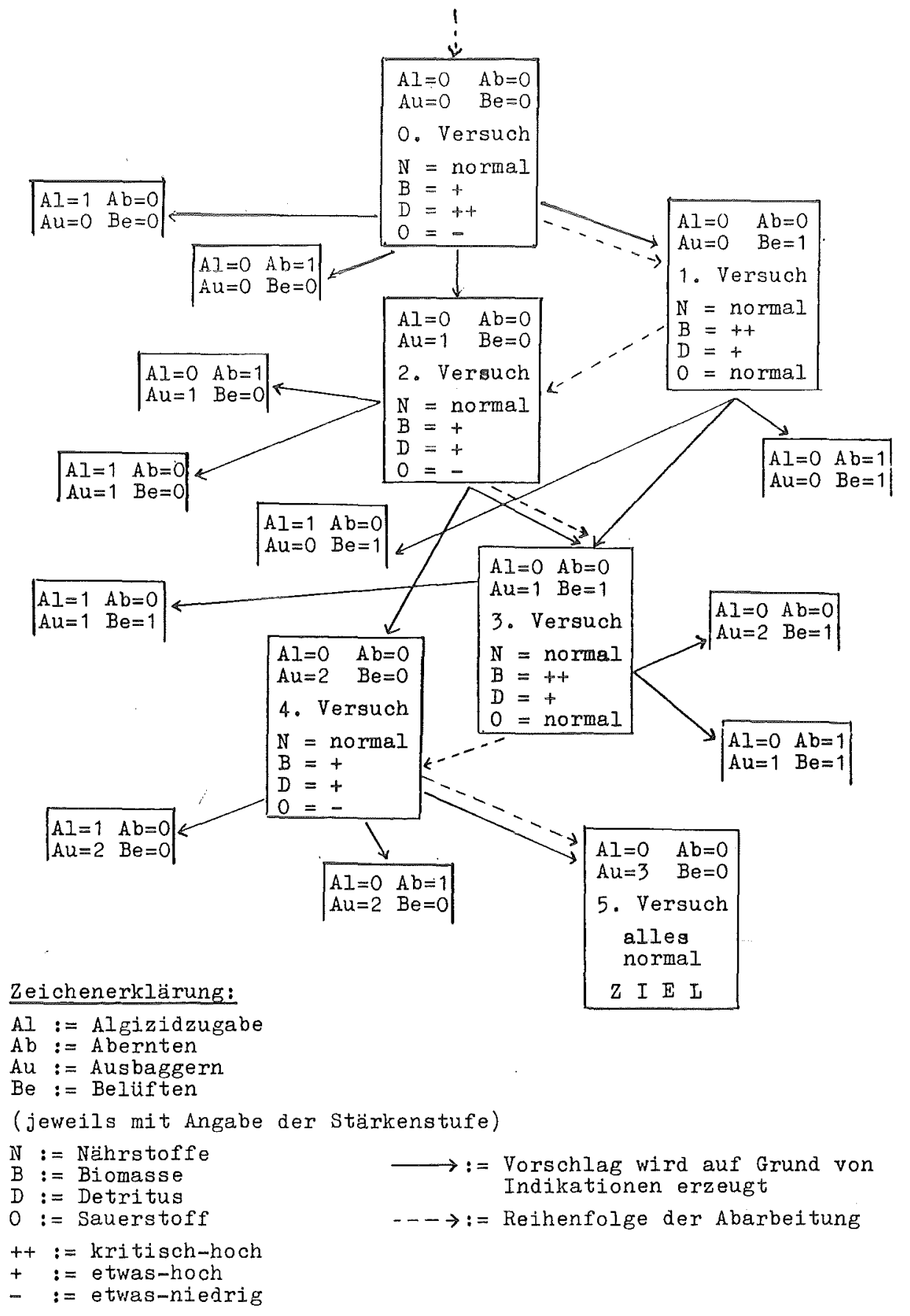

Abb. 2: Suchgraph und Bewertung für einen Simulationslauf 
Die möglichen Strategien zur MaBnahmenbewertung stellen prozedurales Wissen ueber die AngepaBtheit an das Problem und den möglichen Erfolg von MaBnahmenkombinationen dar. Der Benutzer muB sich anfangs für eine der implementierten Strategien entscheiden.

Das dynamische Maßnahmenpool enthält alle erzeugten, noch durch einen Simulationslauf auszuwertende, Vorschläge für anzuwendende Maßnahmen in deklarativer Form.

Die statische Hissensbasis enthält Wissen über die qualitative Bewertung quantitativer Werte von Zustandsvariablen und der Dosierung einzelner Massnahmen, sowie heuristisches Wissen über Grïnde, die für oder gegen die Anwendung einzelner MaBnahmen in Abhängigkeit des Zustandes des Sees sprechen. Beide Arten von Wissen sind in deklarativer Form gegeben. Eine genauere Beschreibung des Systemaufbaus wird an einer anderen Stelle gegeben /BEC87/.

In Abb. 2 sind Aufbau und Abarbeitung eines Suchgraphen eines wissensbasierten Simulationslaufes dargeste11t.

Der Benutzer erhält das folgende Protoko11:

Ab dem 0.0 -ten Jahr bis Ende des 15.0 -ten Jahres wurden folgende Massnahmen angewendet:

- Algizide in Staerke $0.0=$ KEIN ALGIZID

- Abernten in Staerke $0.0=\mathrm{KEIN}^{-}$

- Ausbaggern in Staerke 2.0 = GERING

- Belueften in Staerke $0.0=$ KEIN

Erzielte Simulationsergebnisse:

\begin{tabular}{lllll} 
Jahr & $\begin{array}{l}\text { Naehrstoffe } \\
(\mathrm{mg} / \mathrm{l})\end{array}$ & $\begin{array}{l}\text { Biomasse } \\
(\mathrm{mg} / \mathrm{l})\end{array}$ & $\begin{array}{l}\text { Detritus } \\
(\mathrm{mg} / \mathrm{l})\end{array}$ & $\begin{array}{l}\text { Sauerstoff } \\
(\mathrm{mg} / \mathrm{l})\end{array}$ \\
\hline 15.0 & 36.28 & 0.48 & 5.67 & 7.70 \\
& NORMAL & ETWAS_HOCH & ETWAS_HOCH & NORMAL
\end{tabular}

Weiterfuehrende Vorschlaege:

- AUSBAGGERN

kann den Zustand EIWAS_HOCH von DETRITUS verbessern.

- ABERNTEN

kann den Zustand ETWAS_HOCH von BIOMASSE verbessern.

- ALGIZIDE

kann den Zustand ETWAS HOCH von BIOMASSE verbessern.

Ab dem 0.0 -ten Jahr bis Ende des 15.0 -ten Jahres

wurden folgende Massnahmen angewendet:

- Algizide in Staerke $0.0=$ KEIN_ALGIZID

- Abernten in Staerke $0.0=\mathrm{KEIN}^{-}$

- Ausbaggern in Staerke $3.0=$ MAESSIG

- Belueften in Staerke $0.0=K E I N$ 
Erzielte Simulationsergebnisse:

\begin{tabular}{lllll} 
Jahr & $\begin{array}{l}\text { Naehrstoffe } \\
(\mathrm{mg} / \mathrm{l})\end{array}$ & $\begin{array}{l}\text { Biomasse } \\
(\mathrm{mg} / \mathrm{l})\end{array}$ & $\begin{array}{l}\text { Detritus } \\
(\mathrm{mg} / \mathrm{l})\end{array}$ & $\begin{array}{l}\text { Sauerstoff } \\
(\mathrm{mg} / \mathrm{l})\end{array}$ \\
\hdashline 15.0 & 39.16 & 0.30 & 2.44 & 8.69 \\
& NORMAL & NORMAL & NORMAL & NORMAL
\end{tabular}

Folende Loesung wurde gefunden:

\begin{tabular}{|c|c|c|}
\hline Massnahme & Staerke & $\begin{array}{l}\text { Effekt ueber den } \\
\text { Simulationszeitraum }\end{array}$ \\
\hline Algizide & $\begin{array}{l}0.0 \\
\text { KEIN ALGIZID }\end{array}$ & $\begin{array}{l}0.0 \mathrm{mg} / \mathrm{l} \\
\text { Biomasse abgetoetet. }\end{array}$ \\
\hline Abernten & $\begin{array}{l}0.0 \\
\text { KEIN }\end{array}$ & $\begin{array}{l}0.0 \mathrm{mg} / \mathrm{l} \\
\text { Biomasse abgeerntet. }\end{array}$ \\
\hline Ausbaggern & $\begin{array}{l}3.0 \\
\text { MAESSIG }\end{array}$ & $\begin{array}{l}2.99 \mathrm{mg} / 1 \\
\text { Detritus entfernt. }\end{array}$ \\
\hline Belueften & $\begin{array}{l}0.0 \\
\text { KEIN }\end{array}$ & $\begin{array}{l}0.0 \mathrm{mg} / 1 \\
\text { Sauerstoff zugefuehrt. }\end{array}$ \\
\hline
\end{tabular}

Dadurch wird im letzten Simulationsjahr folgender Zustand erreicht:

\begin{tabular}{|c|c|c|}
\hline Parameter & taet (mg/l) & Zustand \\
\hline Naehrstoffe & 39.16 & normal \\
\hline Biomasse & 0.29 & normal \\
\hline tritus & 2.44 & normal \\
\hline $\begin{array}{l}\text { uerstoff } \\
\text { Hypolimnion }\end{array}$ & 8.69 & norm \\
\hline
\end{tabular}

Damit ist der Zustand des Sees insgesamt NORMAL.

\section{Problematik eines wissensbasierten Ansatzes}

Obwohl mit den vorliegenden System eine oder mehrere Lösungen durch ein Wechselspiel von Simulation, Beurteilung und regelgesteuerter Suche gefunden werden, sind die notwendigen Voraussetzungen eines allgemeineren Einsatzes dieser Methode noch nicht untersucht worden. Erst die Einschränkung des Suchraumes durch starke Diskretisierung ermöglicht die hier beschriebene Anwendung. Das hierbei gewählte Verfahren einer qualitativen Beschreibung wird allerdings auch von den "Experten" zur Beurteilung eines ökosystems verwendet (vgl. /SCH78/, S. 124).

Eine weitere Verbesserung kann sicherlich durch Einbettung von 
Wissen über das Modell in das Expertensystem erreicht werden. Hierzu existieren bereits Vorschläge, eine Realisierung ist bisher noch nicht durchgeführt worden (/SHA85/).

\section{Ausblick für Weiterentwicklungen}

Das bisher beschriebene Programmsystem analysiert den Zustand des Sees statisch vor und nach einer durchgeführten MaBnahme. Ein wichtiges Kriterium zur Bewertung des Sees ist aber auch sein dynamisches Verhalten während eines Simulationslaufes. Ein Expertensystem wird i.a. nicht aus den Werten aller Zustandsvariablen zu allen Beobachtungszeitpunkten Entscheidungen treffen können. Vor den eigentlichen Einsatz des Expertensystems müssen die Daten in eine für das wissensbasierte System geeignetere Darstellung transformiert werden. Hierzu ist eine Abstraktion der Datenmenge des Simulationsprogramens in eine andere Repräsentationsform erforderlich.

Zur Zeit wird ein Ansatz verfolgt, bei dem die Daten des Simulationsprogramens in eine qualitativere Form überführt werden. Die hierbei zugrundeliegenden Konzepte sind auch auf die Beschreibung anderer zeitabhängiger Variablen anwendbar.

Statt der genauen Darstellung der gewählten Repräsentation in der Programmiersprache OPS5 wird hier eine verbale Beschreibung angegeben. Für das wissensbasierte System wird dann weiterhin mit. diesen Beschreibungen gearbeitet, um die Ansätze für das Regelsystem klarer für den Leser zu machen. Bisher wurden folgende Abstraktionen des dynamischen Verhaltens einer Variablen untersucht:

\section{- konstanter Verlauf}

Aus der Gesamtmenge der simulationsdaten einer gemessenen Zustandsvariablen wird auf wenige Datenelemente abstrahiert. Die verbale Beschreibung der Repräsentation lautet dann etwa:

"Variable Detritus bleibt über den simulationszeitraum konstant"

- lineares Verhalten

Es wird eine Geradenapproximation an die MeBwerte durchgeführt, als Datenelemente werden Zeitabschnitte mit linearen Verlauf der Variablen produziert. Qualitative Bewertungen wie Z.B. "stark" oder "schwach" können ebenfalls als domänenspezifisches Wissen durch Angabe der Intervallgrenzen modelliert werden.

- Oszillationen

Oszillationen können nur schwer aus den tabellarischen Simulationsergebnissen erkannt werden, daher werden Simulationsergebnisse zumeist auch in grafischer Darstellung gegeben. Beim hier vorgestellten System werden 0szillationen erkannt und ebenfalls als Datenelemente abgelegt. Daraus können Beschreibungen der Form 
"Variable Nutrient oszilliert zwischen den Jahren 10 und 40 mit einer Maximalamplitude von 7 Einheiten"

- Verdoppelung/Halbierung

Eine weitergehende Abstraktion des Verlaufs einer Meßgröße sind Aussagen der Form

"Der Sauerstoffgehalt verdoppelt sich innerhalb von 10 Jahren"

Derartige Aussagen geben dem Benutzer des Systems einen groben Überblick über die Zustandsveränderungen und können bei späteren Interaktionen des Benutzers mit dem System Anwendung finden ("Wie verhält sich der Sauerstoffgehalt ?").

- Sprungfunktionen

- exponentielles Wachstum

- Vergleich zweier Variablen

Die hier vorgestellten abstrakteren qualitativen Beschreibungen sind sicher noch nicht vollständig, geben aber dennoch einen Überblick über die Möglichkeiten der Beschreibung des Modellverhaltens. Wesentliche Teile werden zur Zeit im Rahmen einer Studienarbeit /NOGARTH86/ implementiert und untersucht.

Mit Hilfe dieser abstrakteren Beschreibungen läßt sich das Expertenwissen über die Vorgänge im See besser beschreiben als durch die Interpretation von Zahlenkolonnen.

Das regelhafte Wissen läBt sich dann in folgender Form in das wissensbasierte System einbringen:

WENN

Maßnahme 〈M〉 ergriffen wurde UND DANN

Zustandsvariable $\langle V\rangle$ sinkt

verwerfe Maßnahme (M)

WENN

Maßnahme 〈M> ergriffen wurde UMD

Zustandsvariable $\langle V 1\rangle$ konstant: UND

Zustandsvariable 〈V2〉 "langsam" sinkt DANN

führe MaBnahme 〈佂〉 "versturkt" fort UND

simuliere weitere 〈n〉 Jahre

Beim Aufbau dieser prozeduralen Wissensbasis ist die Mithilfe eines Experten erforderlich, der in Zusammenarbeit mit dem Entwickler des Expertensystems diese Regeln formuliert und in das Expertensystem einbringt. 


\title{
9. Literaturverzeichnis
}

\author{
/AMD73/ J.M. Anderson \\ The Eutrophication of Lakes \\ in: D.L. Meadows, D.H. Meadows \\ Toward Global Equilibrium \\ Wright-A11en Press, Cambridge, Mass. (1973) \\ /BEC87/ S. Becker, M. Ramlow \\ Graphsuchverfahren zur Optimierung der \\ Ergebnisse eines Simulationsmodells \\ Studienarbeit, Fachbereich Informatik \\ Universität Hamburg, erscheint 1987 \\ /BR085/ L. Brownston, R. Farrel, E. Kant, M. Martin \\ Programining Expert Systems in OPS5 \\ Reading. Mass.: Addison-Wesley (1985) \\ /FOR81/ C.L. Forgy \\ OPS5 User's Manual \\ Report CMU-CS-81-135, Carnegie-Mel1 on University \\ Pittsburgh (1981) \\ /HAY83/ F. Hayes-Roth, D.A. Haterman, D. Lenat \\ Building Expert Systems \\ Reading, Mass.: Addison-Wesley (1983) \\ /AIL80/ \\ N. Nilsson \\ Principles of Artificial Intelligence \\ Springer Verlag, Berlin Heidelberg New York (1980) \\ /N0G87/ L. Nogarth \\ Qualitative Beurteilung zeitkontinuierlicher \\ Variablen zur Wissensrepräsentation in \\ Expertensystemen \\ Studienarbeit, Fachbereich Informatik \\ Universität Hamburg, erscheint 1987 \\ /RED86/ Y.V. Reddy, M.S. Fox, N. Husain, M. McRoberts \\ The Knowledge-Based Simulation System \\ IEEE Sof tware, (März 1986) \\ /SCH74/ E. Schmidt \\ ökosystem See \\ Quelle \& Meyer, Heidelberg (1974) \\ /SHA85/ \\ R.E. Shannon, R. Mayer, H. Adelsberger \\ Expert Systems and Simulation \\ Simulation (1985), 275-284
}


Autorenliste

D. Bartl

Staatsministerium

Rich.-Wagner-Str. 15

7000 Stuttgart

\section{S. Becker}

Universität Hamburg

Fachbereich Informatik

Schlüterstr. 70

2000 Hamburg 13

D. Brune

Kernforschungszentrum Karlsruhe $\mathrm{GmbH}$

AFAS

Postfach 3640

7500 Karlsruhe

Dr. K. Fedra

IIASA

Schloßplatz

A-2361 Laxenburg

W. Gillessen

IABG

Einsteinstr. 20

8012 Ottobrunn

A. Hansen

Universität Hamburg

Fachbereich Informatik

Schlüterstr. 70

2000 Hamburg 13

L. Hilty

Universität Hamburg

Fachbereich Informatik

Schlüterstr. 70

2000 Hamburg 13

Prof. Dr.-Ing. B. Page

Professor für Anwendungen der Informatik

Universität Hamburg

Fachbereich Informatik

Schlüterstr. 70

2000 Hamburg 13

Dr. E. Reichardt

IABG

Einsteinstr. 20

8012 Ottobrunn
A. Rudolf
Dornier GmbH Abt. ZIUB
Postfach 1360
7990 Friedrichshafen 1

\author{
A. Schwabl \\ Universität Hamburg \\ Fachbereich Informatik \\ Schlüterstr. 70 \\ 2000 Hamburg 13
}

Dir. und Prof. Dr. J. Seggelke

Umweltbundesamt

Bismarckplatz 1

1000 Berlin 33

Dr. Stopp

Umweltbundesamt

Bismarckplatz 1

1000 Berlin 33

R. Weidemann

Kernforschungszentrum Karlsruhe $\mathrm{GmbH}$

IDT

Postfach 3640

$7500 \mathrm{Karlsruhe}$ 